\title{
A NEW LIGHT ON PROSTATE CANCER
}

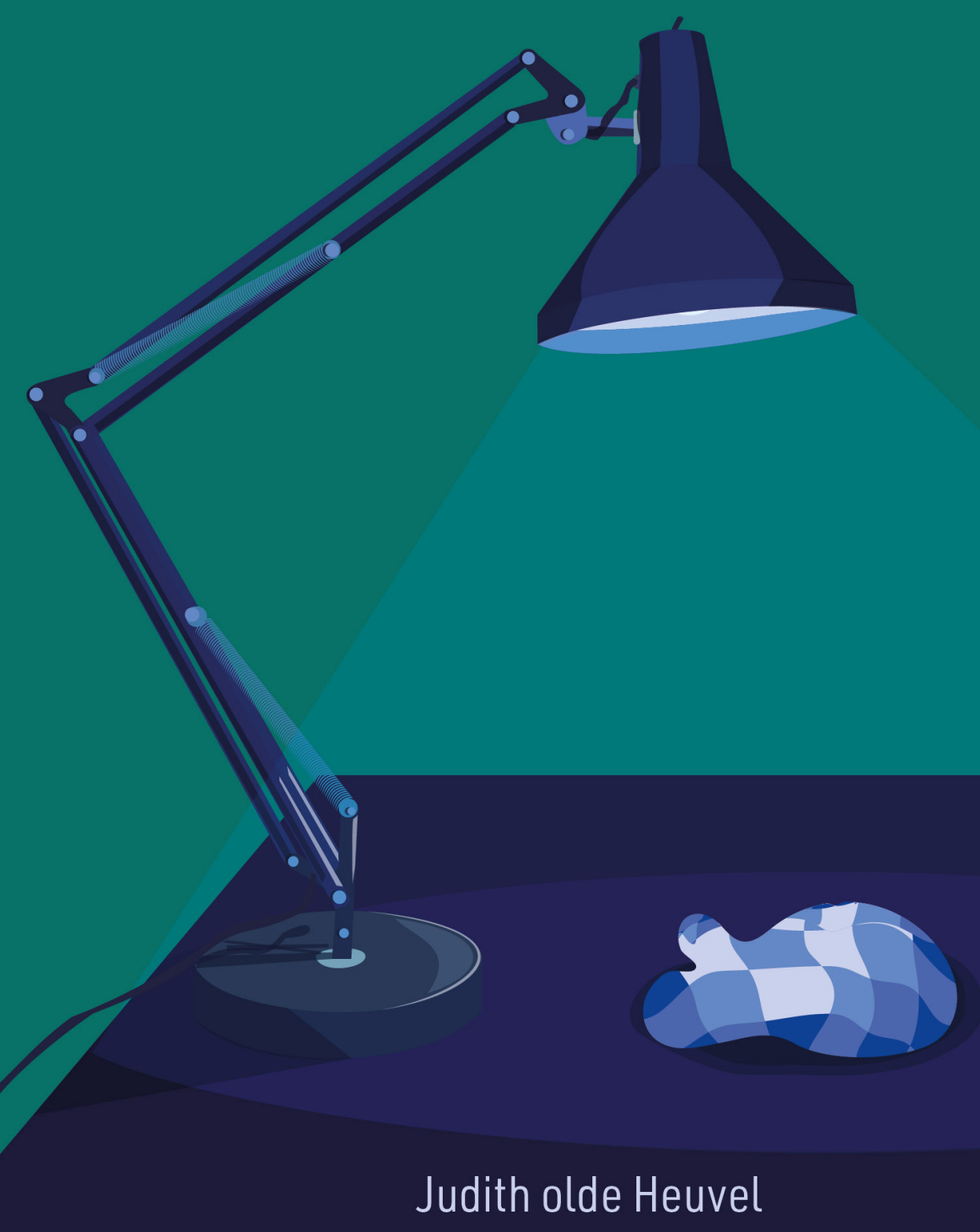



A new light
on prostate cancer

Judith olde Heuvel 
This dissertation has been approved by:

Supervisor

Prof. dr. ir. C.H. Slump

Co-supervisors:

Dr. M.P.M. Stokkel

Dr. B.J. de Wit-van der Veen

Cover design: Berber M. Galema

Printed by: Ridderprint.nl

Lay-out: Berber M. Galema

ISBN: 978-90-365-5128-1

DOI: $10.3990 / 1.9789036551281$

The research conducted in this thesis is supported by KWF Kankerbestrijding and Technology Foundation STW, as part of their joint strategic research programme 'Technology for Oncology' (Grant number 15175).

Financial support for publication of this thesis was provided by LightPoint Medical, the Oncology Graduate School (Netherlands Cancer Institute) and Robotics and Mechatronics (University of Twente)

(c) 2021 Judith olde Heuvel, The Netherlands. All rights reserved. No parts of this thesis

may be reproduced, stored in a retrieval system or transmitted in any form or by

any means without permission of the author. Alle rechten voorbehouden. Niets uit

deze uitgave mag worden vermenigvuldigd, in enige vorm of op enige wijze, zonder

voorafgaande schriftelijke toestemming van de auteur 


\title{
A NEW LIGHT ON PROSTATE CANCER DISSERTATION
}

\author{
To obtain \\ the degree of doctor at the University of Twente, \\ on the authority of the rector magnificus, \\ prof. dr. ir. A. Veldkamp \\ on account of the decision of the Doctorate Board \\ to be publicly defended
} on Friday 26 March 2021 at 12:45 hours

by

Judith olde Heuvel

born on the 4th of January 1992

in Hoogeveen, the Netherlands 



\section{Graduation Committee}

Chair / secretary: Prof. dr. J.N. Kok

Supervisor: $\quad$ Prof. dr. ir. C.H. Slump

Co-supervisors: Dr. M.P.M. Stokkel

Dr. B.J. de Wit-van der Veen

Committee Members: $\quad$ Prof. dr. I.A.M.J. Broeders

Prof. dr. J.J. Fütterer

Prof. dr. L.F. de Geus-Oei

Prof. dr. R. de Bree

Dr. D.E. Oprea-Lager 


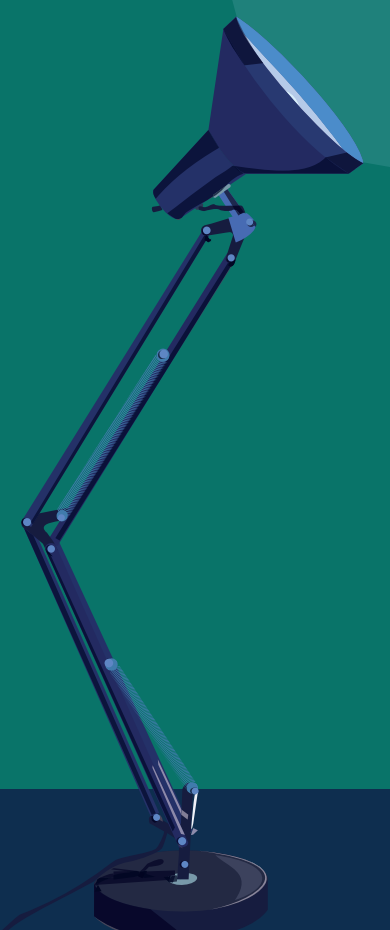




\section{CONTENTS}

Chapter 1 GENERAL INTRODUCTION AND OUTLINE

Part I Requirements for intraoperative margin assessment in prostate cancer surgery

Chapter 2 STATE-OF-THE-ART INTRAOPERATIVE IMAGING TECHNOLOGIES FOR PROSTATE MARGIN ASSESSMENT: A SYSTEMATIC REVIEW

Chapter 3 PERFORMANCE EVALUATION OF CERENKOV LUMINESCENCE IMAGING:

Part II

Chapter 4 DAY-TO-DAY VARIABILITY OF 68GA-PSMA-II ACCUMULATION IN PRIMARY PROSTATE CANCER: EFFECTS ON TRACER UPTAKE AND VISUAL INTERPRETATION

Chapter 5 EARLY DIFFERENCES IN DYNAMIC UPTAKE OF ${ }^{\circ}$ GA-PSMA-1I IN PRIMARY PROSTATE CANCER: A TEST-RETEST STUDY

Chapter $6 \quad{ }^{68}$ GA-PSMA CERENKOV LUMINESCENCE IMAGING IN PRIMARY PROSTATE CANCER: FIRST-IN-MAN SERIES

Chapter 7 CERENKOV LUMINESCENCE IMAGING IN PROSTATE CANCER:

NOT THE ONLY LIGHT THAT SHINES

Chapter 8 GENERAL DISCUSSION AND FUTURE PERSPECTIVES

Chapter 9 SUMMARY SAMENVATTING

Appendices

LIST OF PUBLICATIONS

LIST OF AFFILIATIONS

DANKWOORD 


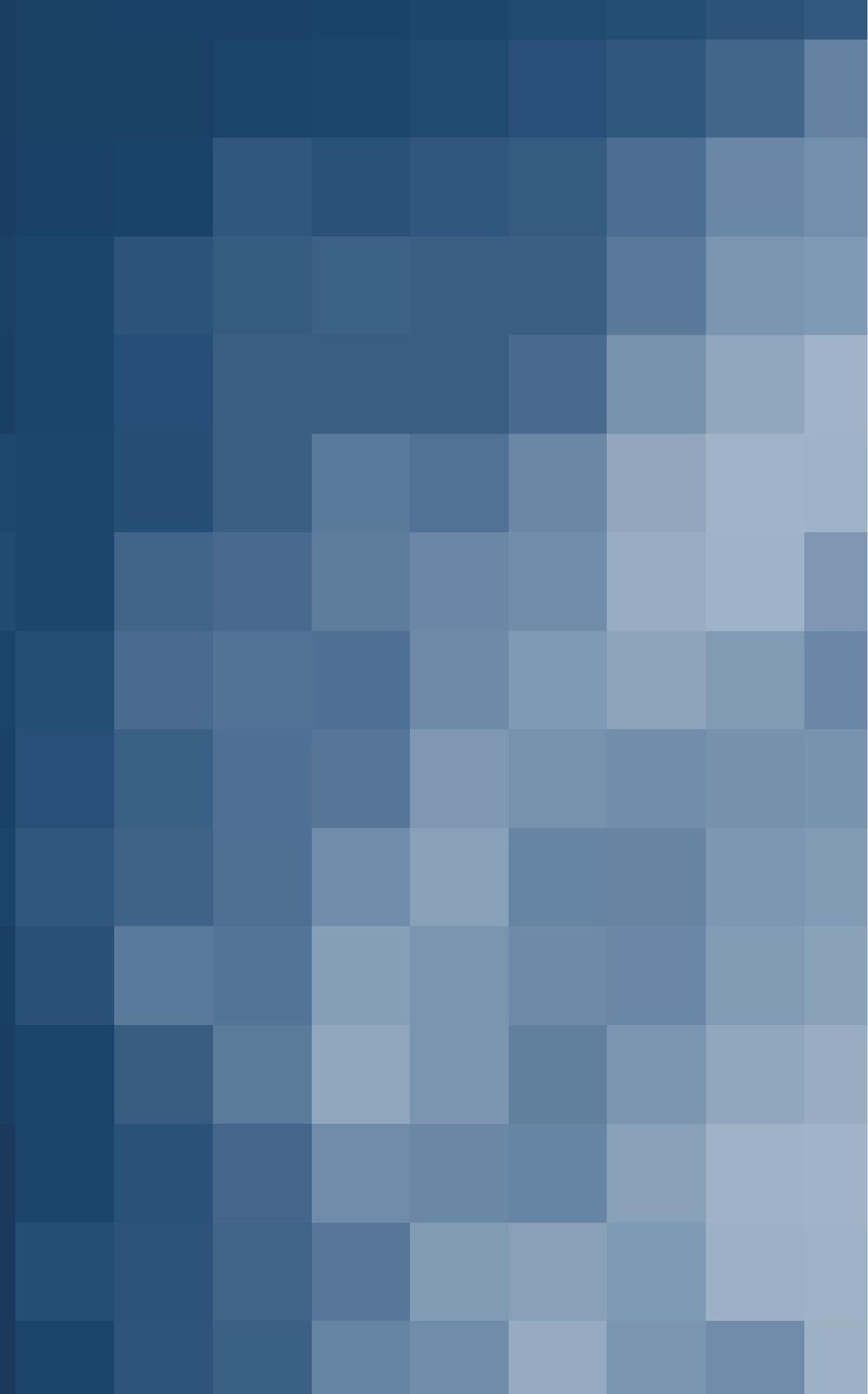




\section{CHAPTER 1}

GENERAL INTRODUCTION AND OUTLINE 


\section{General introduction}

\section{PROSTATE CANCER}

Prostate cancer (PCa) is the second most common cancer in males in the Netherlands, with approximately 12.000 new cases diagnosed each year [1]. Patients with metastases at diagnosis (15\% of the cases at initial diagnosis) have a relatively low 10 -year survival rate of $25 \%$, whereas patients with localized PCa ( 60\% of the cases) have a 10-year survival rate of $>95 \%$. In patients with locally advanced disease (20\% of the cases), the 10 -year survival rate is $85 \%$ [1]. Nevertheless, in about a quarter of patients treated with local curative intent, the disease will recur in time. Henceforth, it is important to further optimize the strategies for diagnosis and treatment of primary PCa.

\section{Zones of the prostate}

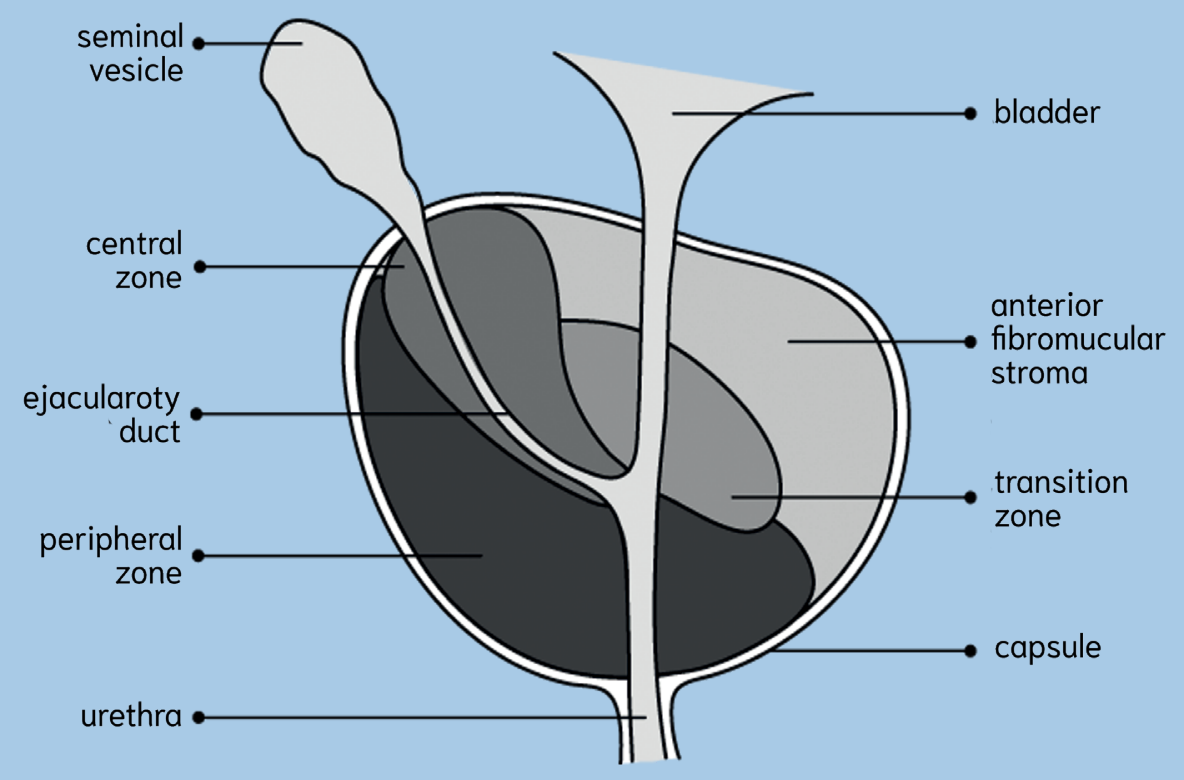

Figure 1. Schematically overview of the anatomy of the prostate and the zones within left of the urethra is the posterior side and right is the anterior side. Obtained from the Canadian Cancer Society [2].

\section{Clinical presentation and diagnosis}

Patients with prostate cancer usually do not have symptoms in an early stage, as most of the tumours are located in the peripheral zone, see Figure 1. In some cases the tumour is large enough to oppress the urethra, and patients might suffer from lower urinary tract symptoms (LUTS), like obstruction, nocturia and a weak urinary flow. 
The diagnosis of PCa is based on different examinations. A digital rectal examination (DRE) is performed to assess the size and consistency of the prostate, however, it has a low predictive value in the diagnosis of prostate cancer $[3,4]$. In addition, the prostate-specific antigen (PSA) level in blood samples is obtained. Although this test is very sensitive, the specificity is low. A high PSA level can indicate prostate cancer, prostatitis, as well as benign prostate hyperplasia. A (ultrasound-guided) prostate biopsy is obtained to determine the Gleason score, which represents the architectural features of prostate cancer cells. The result of a biopsy is predictive for pathological staging and progression free survival [5,6]. Multi-parametric magnetic resonance imaging (mpMRI) is advised for staging the tumour extent, and sometimes to indicate lesions for target biopsies. Based on clinical staging (DRE and biopsies) and imaging results, the TNM classification of malignant tumours is determined. The TNM-stage, the Gleason score and the PSA level divide the PCa into risk profiles: local disease (subdivided into low, intermediate and high-risk) [7], locally advanced and metastatic disease.

\section{PET/CT imaging}

In patients with high risk $\mathrm{PCa}$, additional imaging is indicated to assess possible lymph node involvement or distant metastasis. Traditionally this was assessed using a bone scintigraphy combined with a diagnostic $\mathrm{CT}$, but nowadays positron emission tomography (PET) has obtained a prominent role in the Netherlands in this respect. In the recent years, new PET-tracers were introduced to detect prostate cancer, targeting the prostate-specific membrane antigen (PSMA). PSMA is a transmembrane protein, a folate hydrolase cell surface glycoprotein, which is also expressed in benign tissue and other organs. Still, the overexpression of PSMA is 100-1000 times higher in malignant lesions as compared to benign tissue, see Figure 2. The PSMA ligand can both be labelled to fluorine-18 $\left({ }^{18} \mathrm{~F}\right)$ as well as to gallium-68 $\left({ }^{68} \mathrm{Ga}\right)$. PSMA has shown unprecedented added value in detecting metastatic prostate cancer and local recurrence $[8,9]$, where even in patients with low PSA levels the lesions can be detected. Next, PSMA has a higher sensitivity than a bone scan for patients in the metastatic setting [10].

Additionally, PSMA PET/CT scan is deployed as well to stage the primary PCa. Since it has a higher diagnostic accuracy in detecting the presence of lymph node involvement and distant metastasis, as compared to conventional imaging (CT and bone scan) [11-14]. Besides, the use of PSMA PET/CT scan may lead to changed N and $M$ stages, resulting in other treatment strategies in up to $36 \%$ of the patients compared to the treatment suggestion prior to PSMA PET/CT scan $[15,16]$. Hence, the PSMA PET/CT scan is nowadays advised in the Dutch Guideline for Prostate Cancer (version 2.3) in men with a high risk on metastasis, these men have a stage $\geq c T 3$ and/or PSA $\geq 20 \mathrm{ng} / \mathrm{ml}$ and/or Gleason Score of $\geq 8$ [17].

In general, PSMA PET/CT scans are evaluated with visual assessment performed by a nuclear medicine physician; however, (semi-) quantitative measurements can contribute valuable information. The latter is often performed by using the metric standardized uptake value (SUV), which generally represents the radioactivity con- 
centration in an area, corrected for the injected dose and the patient's body weight. In ${ }^{18} \mathrm{~F}$-FDG ( ${ }^{18} \mathrm{~F}$-fluorodeoxyglucose) PET/CT scans the SUV value is also used for response assessment in several tumour types [18], as an increase in uptake is associated with disease progression. However, the use of the SUV metrics in PSMA-PET for response assessment and tumour aggressiveness in prostate cancer is still under development.
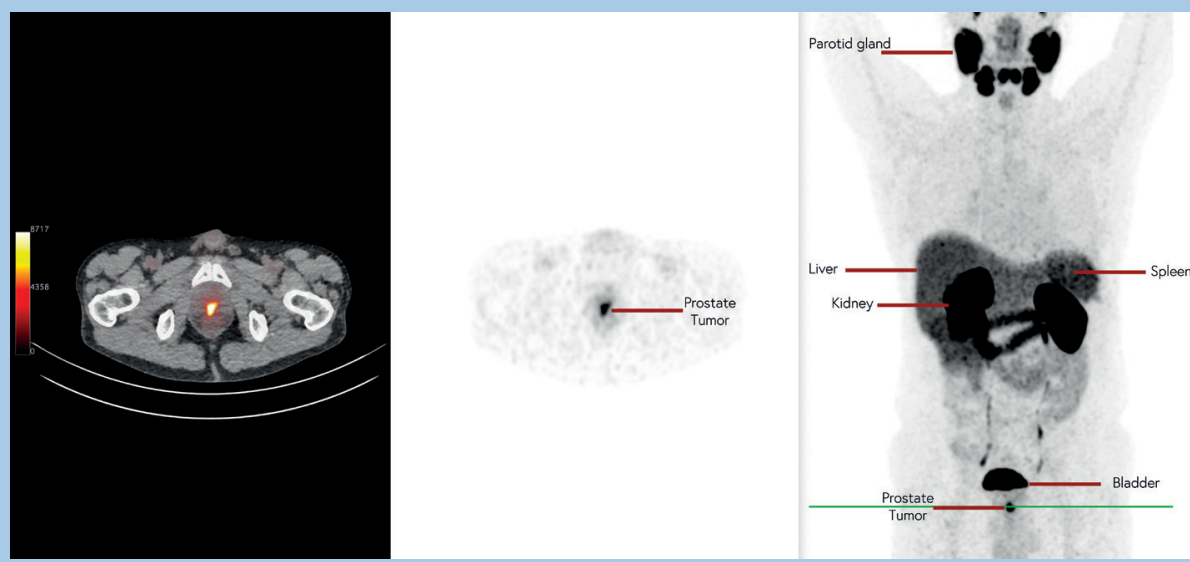

Figure 2. An example of a ${ }^{68} \mathrm{Ga}-\mathrm{PSMA}-11 \mathrm{PET/CT}$ scan. A cross section of the prostate tumour (see green line on the right] is visible on the fused PET/CT scan on the left, and on the PET scan in the centre. Physiological high uptake can be observed in the glands, liver, spleen and kidneys. ${ }^{68} \mathrm{Ga}-\mathrm{PSMA}-11$ is excreted via the urinary tract.

Therapy for patients with primary PCa

The TNM-stage of PCa influences the choice of treatment, together with the preference of the patient. Of all patients diagnosed with primary $\mathrm{PCa}$, about $33 \%$ will undergo a radical prostatectomy [1]. Prostatectomy or radiotherapy are frequently performed in patients with limited local disease (T1-T2), however, in some cases active surveillance is preferred, which involves frequent PSA tests, DRE, imaging and biopsies to monitor the primary tumour. In patients with locally advanced disease (T3-T4), about half of the patients will be treated with radiotherapy in combination with hormonal treatment. In other patients, surgical treatment is still an option, depending on the prognostics of the disease and the potential comorbidities of the patient. In case of metastatic disease there are only palliative options, which include hormonal therapy, sometimes in combination with chemotherapy or radiotherapy, to inhibit disease progression. However, after 1-2 years of palliative treatment the PCa will become castrate resistant, resulting in disease progression [19].

\section{Radical prostatectomy}

In primary $\mathrm{PCa}$, a radical prostatectomy (RP) can be performed with a curative intent, through open surgery or as a robotic-assisted laparoscopic prostatectomy. Robotic surgery has some advantages in comparison to open surgery, such as less 
side effects and shorter hospitalization duration $[20,21]$. With this minimally invasive technique and optical magnification of the surgical field the entire prostate and seminal vesicles are removed. In case there is a high risk of lymph node involvement, based on the Briganti nomogram [22], (extended) pelvic lymph node dissection can be performed as well during surgery.

After a RP the entire prostate is send to pathology for final histopathological examination after the surface is inked to persevere orientation, i.e. black for the right side and green for the left. This examination comprises an assessment of TNM classification, Gleason score, extra prostatic extension, invasion of the seminal vesicles and surgical margins [23]. The latter is performed to check how radical the surgery was performed. In case of a negative surgical margin (NSM), the tumour is removed with $\geq 1$ cell-layer distance from the surface. In case of a positive surgical margin (PSM), there are still tumour cells present on the inked surface [23], see Figure 3. This means that there is an increased risk that tumour cells are still present in the surgical field, indicating that the surgery was not radical.

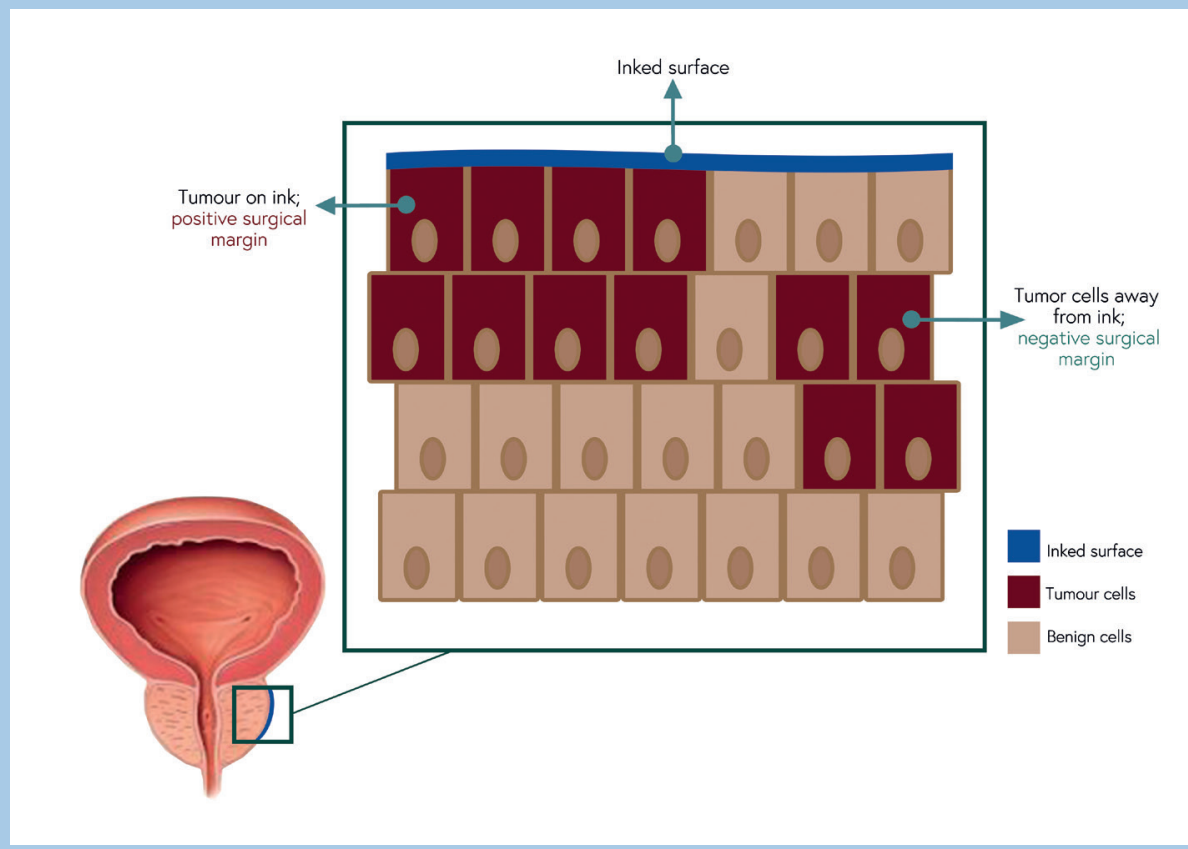

Figure 3. A schematic representation of a positive surgical margin and a negative surgical margin. The blue line represents the ink on the prostate. The red cells represent tumour cells, as the grey cells are benign. If the tumour cells touch the ink, this is considered to be a PSM. Whereas if there is one layer of benign cells between the tumour cell and the ink, a negative margin is given.

The risk of a PSM depends on different factors. First of all, the stage of the primary tumour, in other words the extension of cancer in the gland. On average, PSMs occur in about $20 \%$ of the cases $[24,25]$, whereas in T2 the rates are about $10 \%$ increasing 
to $40 \%$ in T3-tumours [26]. Large tumours (T3-T4) are generally closer to the edge of the gland and therefore more prone to result in a PSM. Secondly, more experienced surgeons are less likely to provoke a PSM [27]. Finally, the PSM incidence depends on the location of the tumour, this is higher in tumours located near the apex, the neural vascular bundle or at the base of the prostate $[28,29]$. As most tumours are located in the apex, most PSMs occur over there. Besides, as the base and the apex of the prostate do not have a clear 'boundary', it is more likely to cause a PSM at these sides. In tumour located near the neurovascular bundle, there is a trade-off between preserving as much nerves for erectile function while not invoking a PSM. In other words, successful surgery is established by both securing good therapeutic outcome and preserving sexual function and continence, the so-called trifecta [30].

\section{Consequences of a positive surgical margin}

The impact of a PSM remains controversial, and it does not mean disease recurrence by definition [31]. In some literature, a PSM is associated with a worse prognostic outcome [32,33], and it is described as a statistically independent significant predictor of recurrence $[34,35]$. In contrast, in some studies it has been reported that a PSM does not affect the progression rate, compared to those with a NSM [36,37]. In other words, a PSM has low predictive value for tumour recurrence [31].

However, this does not mean that margin status can be neglected. In some patients adjuvant external radiotherapy is advised to improve progression free survival $[38,39]$. Additionally, it has a psychological burden on a patient, when he knowns 14 that there are potentially cancer cells left behind in his body. As a PSM has such an influence on the life of patients and the medical expenses, it is important to strive for a reduction of the PSM-rate, which might be achieved with technological advances in intraoperative guidance to aid a radical resection.

One of the options to perform perioperative surgical margin assessment is the use of frozen section analysis [40]. With this technique, areas of suspicion are send to pathology during surgery for margin assessment. The result will decide if a re-resection is needed to convert a PSM into a NSM. Still, this technique is labour- and time intensive and prone to sampling errors. Thus, the search towards intraoperative technologies that can guide a more radical resection continues.

\section{CeRENKOV LUMINESCENCE IMAGING}

\section{Principle of Cerenkov radiation}

An emerging technology that might be used to assess surgical margins intraoperatively is Cerenkov luminescence imaging (CLI). It is thought that Marie Curie is the first to describe the Cerenkov phenomenon in the $19^{\text {th }}$ century, as she writes about a blue glow coming from one of the radium-containing bottles. However, Pavel Cerenkov is in 1934 the first one who systematically describes the blue light [41].

Cerenkov radiation is induced when a charged particle (positron/electron) travels faster than the velocity of light in that specific dielectric medium. As a result, it in- 
duces a polarization by displacing the atoms in the medium. This results in an asymmetrical polarization, which causes a dipole electric field. As the charged particle moves through the tissue, the atoms return to their ground state. The difference in energy between these states, is emitted as optical photons, also known as Cerenkov radiation (Figure 4) [42]. In other words, the Cerenkov radiation is produced by the medium as reaction to the charged particle, not by the charged particle itself; it is a so-called secondary emission [43]. The Cerenkov spectrum includes the entire range of 350-900 nm, but has its peak in the ultra-violet blue region [44].

Cerenkov radiation can only be induced if the charged particle has an energy above a certain threshold. Due to the interaction of the charged particle with the surrounding medium, energy is lost which results in an end to the production of Cerenkov radiation. The energy threshold depends on the phase velocity in the medium, and thereby also on the refractive index ( $n)$ of the medium. In water $(n=1.33)$ this threshold is $0.264 \mathrm{MeV}$, whereas in tissue ( $n=1.4)$ this is $0.219 \mathrm{MeV}$ [45]. Consequently, the amount of photons emitted in tissue is higher than in water, as the energy threshold for Cerenkov emission is lower.

CLI images can be acquired by detecting the Cerenkov light from PET tracers using sensitive optical cameras such as electron-multiplying charge-coupled device (emCCD) cameras, one of these devices is shown in Figure 4 [42]. CLI and PET are directly correlated, as both techniques measure photons produced by positron-emitting radiopharmaceuticals; PET measures the annihilation photons, and CLI measures the Cerenkov photons.

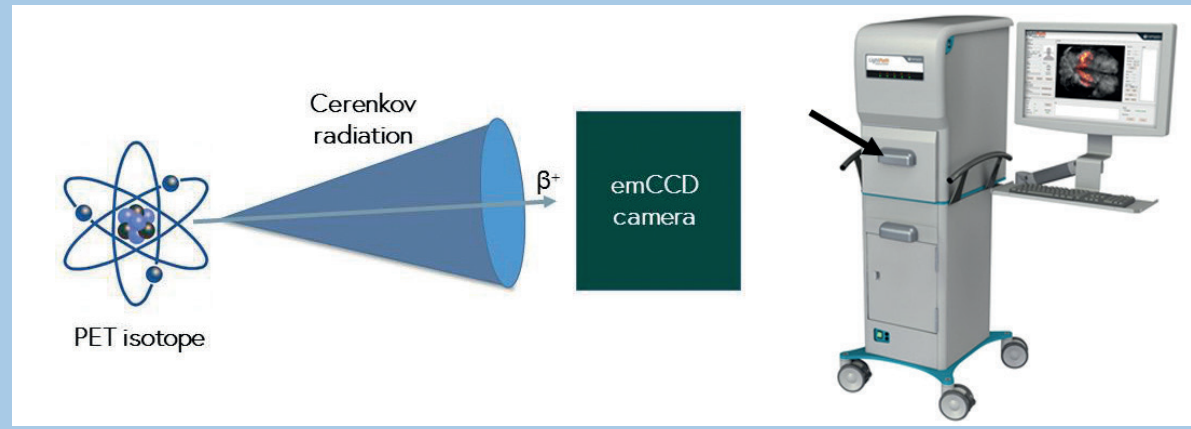

Figure 4. A schematically representation of the principle of Cerenkov radiation. Cerenkov radiation is induced when a charged particle travels faster than the velocity of light in that specific dielectric medium. Resulting in polarization in the tissue. As the charged particle moves further, the atoms return to their ground state thereby emitting Cerenkov photons under a certain angle. On the right a picture of the LightPath system [Lightpoint Medical, Ltd.], this is used to detect Cerenkov radiation by using an EMCCD camera. The black arrow points at the drawer in which the specimen can be placed. 


\section{Clinical application of CLI}

In 2009 the interest in Cerenkov applications was revived, as Robertson et al. was the first to demonstrate the application of PET radiotracers in combination with CLI for imaging cancer in vivo [44]. Since then, multiple Cerenkov applications emerged in the biomedical field such as: photo activation therapy, Cerenkov luminescence imaging dosimetry, radionuclide therapy monitoring [46-49]. CLI allowed rapid translation from pre-clinical into clinical practice, as it uses registered clinical radiopharmaceuticals. Thereby allowing dual-modality molecular imaging, with preoperative PET scans for localization of the lesion, and for example intraoperative information with CLI to guide surgical resection [43]. In 2018, a first clinical trial with CLI was conducted in breast conserving surgery, where CLI was used with ${ }^{18} \mathrm{~F}-\mathrm{FDG}$ to assess the resection margin intraoperatively. This study showed that the application of CLI technology was feasible and it provided promising first results [50].

\section{IMAGE-GUIDED MARGIN ASSESSMENT DURING PROSTATECTOMY}

Despite technical advances in surgery, irradical resection of PCa still occurs frequently as it is difficult to distinguish between malignant and benign tissue intraoperatively only with palpation and visual inspection. PSMs are associated with a higher risk on recurrences and subsequent adjuvant therapy, impacting the quality of life of the patient. At the same time, diagnostic imaging of PCa has progressed dramatically over the last five years, with the introduction of specific tumour targeting tracers. It is hypothesized that combining these tracers with novel imaging sys16 tem, such as CLI, might assist the surgeon with a radical excision and thus reduce PSM rate. In this thesis, the application of PSMA-directed CLI is introduced to shine a new light on prostate cancer surgery. 


\section{Outline of this thesis}

The general aim of the research in this thesis is to investigate the feasibility of ${ }^{68} \mathrm{Ga}-\mathrm{PSMA}-11$ as intraoperative margin assessment technology in prostate cancer surgery. This research includes both the pre-clinical in vitro performance evaluation of the technique, as well as a clinical feasibility trial. In addition, repeatability of the ${ }^{68} \mathrm{Ga}$-PSMA-11 PET/CT in both static and dynamic PET acquisitions was investigated to determine uptake patterns and usability of preoperative ${ }^{68} \mathrm{Ga}-\mathrm{PSMA}-11 \mathrm{PET} / \mathrm{CT}$ in the CLI workflow.

Part I of this thesis focusses on the application of and requirements for intraoperative margin assessment techniques in prostate cancer surgery. Chapter $\mathbf{2}$ is a systematic review of the different options for intraoperative margin assessment in the operating room. The technical background of different methods is provided, the application in prostate cancer surgery, and the advantages and drawbacks for clinical implementation. Prior to investigating the feasibility of intraoperative margin assessment using CLI, the performance of ${ }^{68} \mathrm{Ga}-P S M A-11$ for $\mathrm{CLI}$ needs to be evaluated. So far, $\mathrm{CLI}$ is only used with ${ }^{18} \mathrm{~F}-\mathrm{FDG}$, thus a comparison of the performance with ${ }^{68} \mathrm{Ga}-\mathrm{PSMA}-11$ was executed in Chapter 3 . This chapter further outlines the requirements for ex vivo usage of ${ }^{68} \mathrm{Ga}-\mathrm{PSMA}-11$ in humans, based on in vitro results.

Part II of this thesis introduces the use of ${ }^{68} \mathrm{Ga}-\mathrm{PSMA}-11 \mathrm{PET} / \mathrm{CT}$ in primary PCa for CLI optimization. In current clinical practise, patients undergo a diagnostic PSMA PET/CT scan and prostatectomy is scheduled approximately 4 to 6 weeks later. Since patients are included for ${ }^{68} \mathrm{Ga}-P S M A-11 \mathrm{CLI}$ based on their preoperative PSMA PET/CT scan, similar uptake at the time of surgery needs to be ensured. Therefore, repeatability of ${ }^{68} \mathrm{Ga}$-PSMA-11 uptake was investigated in a 4-week interval, which is described in Chapter 4. Next, in ${ }^{68} \mathrm{Ga}-\mathrm{PSMA}-11$ PET/CT scans the optimal time between injection and imaging is based on the contrast of tumour uptake and activity distribution in the rest of the body. It is unknown whether the same time point is required for CLI imaging, as with CLI only the contrast between benign and tumour tissue in the prostate is relevant for ex vivo imaging. Chapter 5 describes dynamic ${ }^{68} \mathrm{Ga}-\mathrm{PSMA}-11$ PET/CT scans in test-retest setting to evaluate the repeatability of early uptake in the prostate.

Part III explores the use of ${ }^{68} \mathrm{Ga}$-PSMA-11 CLI, as an intraoperative margin assessment technology. Intraoperative detection of PSM might aid a radical excision, thus improving the patients' outcome. To investigate the feasibility of the technique, the results of the first five patients included in the CLI study are discussed in Chapter 6. This interim analysis showed that CLI is feasible and safe for intraoperative application. Important clinical knowledge was acquired necessary to optimize the acquisition protocol and workflow. The study was continued and the results of $\mathrm{CLI}$ accuracy compared to histopathology are reported in Chapter 7. Furthermore, this chapter describes and characterizes a newly identified bioluminescence signal, which might influence the interpretation of the CLI images. 
This thesis ends with a discussion and the future perspectives in Chapter $\mathbf{8}$, followed by summaries in English and Dutch in Chapter 9 . 


\section{REFERENCES}

1. Netherlands Cancer Registry. Managed by IKNL, March 2018. www.cijfersoverkanker.nl.

2. Society CC. The prostate - Canadian Cancer Society. https://www.cancer.ca/en/cancer-information/cancer-type/prostate/prostate-cancer/the-prostate/?region=on.

3. Schröder FH, Van Der Maas P, Beemsterboer P, et al. Evaluation of the digital rectal examination as a screening test for prostate cancer. J Natl Cancer Inst. 1998;90:1817-1823.

4. Richie JP, Catalona WJ, Ahmann FR, et al. Effect of patient age on early detection of prostate cancer with serum prostate-specific antigen and digital rectal examination. Urology. 1993;42:365-374.

5. Freedland SJ, Csathy GS, Dorey F, et al. Percent prostate needle biopsy tissue with cancer is more predictive of biochemical failure or adverse pathology after radical prostatectomy than prostate specific antigen or Gleason score. J Urol. 2002;167:516-520.

6. Kattan MW, Eastham JA, Wheeler TM, et al. Counseling men with prostate cancer: A nomogram for predicting the presence of small, moderately differentiated, confined tumors. J Urol. 2003;170:1792-1797.

7. Ash D, Flynn A, Battermann J, et al. ESTRO/EAU/EORTC recommendations on permanent seed implantation for localized prostate cancer. Radiother Oncol. 2000;57:315-321.

8. Afshar-Oromieh A, Avtzi E, Giesel FL, et al. The diagnostic value of PET/CT imaging with the ${ }^{68} \mathrm{Ga}$-labelled PSMA ligand HBED-CC in the diagnosis of recurrent prostate cancer. Eur J Nucl Med Mol Imaging. 2014;42:197-209.

9. Afshar-Oromieh A, Zechmann CM, Malcher A, et al. Comparison of PET imaging with a ${ }^{68} \mathrm{Ga}-\mathrm{la}-$ belled PSMA ligand and ${ }^{18} \mathrm{~F}$-choline-based PET/CT for the diagnosis of recurrent prostate cancer. Eur J Nucl Med Mol Imaging. 2014;41:11-20.

10. Lengana T, Lawal IO, Boshomane TG, et al. ${ }^{68} \mathrm{Ga}$-PSMA PET/CT Replacing Bone Scan in the Initial Staging of Skeletal Metastasis in Prostate Cancer: A Fait Accompli? Clin Genitourin Cancer. 2018;16:392-401.

11. Hofman MS, Lawrentschuk N, Francis RJ, et al. Prostate-specific membrane antigen PET-CT in patients with high-risk prostate cancer before curative-intent surgery or radiotherapy (proPSMA): a prospective, randomised, multicentre study. Lancet. 2020;395:1208-1216.

12. Maurer T, Gschwend JE, Rauscher I, et al. Diagnostic Efficacy of ${ }^{68} \mathrm{Gallium}$-PSMA Positron Emission Tomography Compared to Conventional Imaging for Lymph Node Staging of 130 Consecutive Patients with Intermediate to High Risk Prostate Cancer. J Urol. 2016;195:1436-1443.

13. Herlemann A, Wenter V, Kretschmer A, et al. ${ }^{68} \mathrm{Ga}$-PSMA Positron Emission Tomography/Computed Tomography Provides Accurate Staging of Lymph Node Regions Prior to Lymph Node Dissection in Patients with Prostate Cancer. Eur Urol. 2016;70:553-557.

14. Corfield J, Perera M, Bolton D, et al. ${ }^{68} \mathrm{Ga}$-prostate specific membrane antigen (PSMA) positron emission tomography (PET) for primary staging of high-risk prostate cancer: a systematic review. World J Urol. 2018;36:519-527.

15. Donswijk ML, Leeuwen PJ van, Vegt E, et al. Clinical impact of PSMA PET/CT in primary prostate cancer compared to conventional nodal and distant staging: a retrospective single center study. BMC Cancer. 2020:20;723.

16. Han S, Woo S, Kim YJ, et al. Impact of ${ }^{68} \mathrm{Ga}$-PSMA PET on the Management of Patients with Prostate Cancer: A Systematic Review and Meta-analysis. Eur Urol. 2018;74:179-190.

17. Nederlandse Vereniging voor Urologie. Prostaatcarcinoom Landelijke richtlijn, Versie: 2.2. 2019. 
18. Wahl RL, Jacene H, Kasamon Y, et al. From RECIST to PERCIST: Evolving Considerations for PET Response Criteria in Solid Tumors. J Nucl Med. 2009;50:122S-150S.

19. Sharifi N, Gulley JL, Dahut WL. Androgen Deprivation Therapy for Prostate Cancer. JAMA. 2005;294:238-244.

20. Tewari A, Sooriakumaran P, Bloch DA, et al. Positive surgical margin and perioperative complication rates of primary surgical treatments for prostate cancer: A systematic review and meta-analysis comparing retropubic, laparoscopic, and robotic prostatectomy. Eur Urol. 2012;62:1-15

21. Novara G, Ficarra V, Mocellin S, et al. Systematic Review and Meta-analysis of Studies Reporting Oncologic Outcome After Robot-assisted Radical Prostatectomy. Eur Urol. 2012;62:382404.

22. Briganti A, Larcher A, Abdollah F, et al. Updated nomogram predicting lymph node invasion in patients with prostate cancer undergoing extended pelvic lymph node dissection: The essential importance of percentage of positive cores. Eur Urol. 2012;61:480-487.

23. Tan PH, Cheng L, Srigley JR, et al. International Society of Urological Pathology (ISUP) consensus conference on handling and staging of radical prostatectomy specimens. Working group 5: surgical margins. Mod Pathol. 2011;24:48-57.

24. Stephenson AJ, Wood DP, Kattan MW, et al. Location, Extent and Number of Positive Surgical Margins Do Not Improve Accuracy of Predicting Prostate Cancer Recurrence After Radical Prostatectomy. J Urol. 2009;182:1357-1363.

25. Yossepowitch O, Briganti A, Eastham JA, et al. Positive Surgical Margins After Radical Prostatectomy: A Systematic Review and Contemporary Update. Eur Urol. 2014;65:303-313.

26. Evans SM, Millar JL, Frydenberg M, et al. Positive surgical margins: Rate, contributing factors and impact on further treatment: Findings from the Prostate Cancer Registry. BJU Int. 2014;114:680-690.

27. Thompson JE, Egger S, Böhm M, et al. Superior Biochemical Recurrence and Long-term Quality-of-life Outcomes Are Achievable with Robotic Radical Prostatectomy After a Long Learning Curve-Updated Analysis of a Prospective Single-surgeon Cohort of 2206 Consecutive Cases. Eur Urol. 2018;73:664-671.

28. Eastham JA, Kuroiwa K, Ohori M, et al. Prognostic Significance of Location of Positive Margins in Radical Prostatectomy Specimens. Urology. 2007;70:965-969.

29. Koskas $Y$, Lannes F, Branger N, et al. Extent of positive surgical margins following radical prostatectomy: Impact on biochemical recurrence with long-term follow-up. BMC Urol. 2019;19:1-8.

30. Bianco FJ, Scardino PT, Eastham JA. Radical prostatectomy: Long-term cancer control and recovery of sexual and urinary function ("trifecta"). Urology. 2005;66:83-94.

31. Vis AN, Schroder FH, van der Kwast TH. The actual value of the surgical margin status as a predictor of disease progression in men with early prostate cancer. Eur Urol. 2006;50:258-265.

32. Epstein JI, Partin AW, Sauvageot J, et al. Prediction of Progression Following Radical Prostatectomy. Am J Surg Pathol. 1996;20:286-292.

33. Connolly SS, O'Toole GC, O'Malley KJ, et al. Positive Apical Surgical Margins after Radical Retropubic Prostatectomy, Truth or Artefact? Scand J Urol Nephrol. 2004;38:26-31.

34. Swindle P, Eastham JA, Ohori M, et al. Do margins matter? The prognostic significanse of positive surgical margins in radical prostectomy specimens. J Urol. 2005;174:903-907.

35. Karakiewicz PI, Eastham JA, Graefen M, et al. Prognostic impact of positive surgical margins in surgically treated prostate cancer: Multi-institutional assessment of 5831 patients. Urology. 
2005;66:1245-1250.

36. Ohori M, Abbas F, Wheeler TM, et al. Pathological features and prognostic significance of prostate cancer in the apical section determined by whole mount histology. J Urol. 1999;161:500504.

37. Van den Ouden D, Bentvelsen FM, Boeve ER, et al. Positive Margins after Radical Prostatectomy: Correlation with Local Recurrence and Distant Progression. Br J Urol. 1993;72:489-494.

38. Valicenti RK, Thompson I, Albertsen P, et al. Adjuvant and salvage radiation therapy after prostatectomy: American society for radiation oncology/american urological association guidelines. Int J Radiat Oncol Biol Phys. 2013;86:822-828.

39. Bolla M, van Poppel H, Tombal B, et al. Postoperative radiotherapy after radical prostatectomy for high-risk prostate cancer: long-term results of a randomised controlled trial (EORTC trial 22911). Lancet. 2012;380:2018-2027.

40. Goharderakhshan RZ, Sudilovsky D, Carroll LA, et al. Utility of intraoperative frozen section analysis of surgical margins in region of neurovascular bundles at radical prostatectomy. Urology. 2002;59:709-714.

41. Čerenkov PA. Visible Radiation Produced by Electrons Moving in a Medium with Velocities Exceeding that of Light. Phys Rev. 1937;52:378-379.

42. Ciarrocchi E, Belcari N. Cerenkov luminescence imaging: physics principles and potential applications in biomedical sciences. EJNMMI Phys. 2017;4:14.

43. Grootendorst MR, Cariati M, Kothari A, et al. Cerenkov luminescence imaging (CLI) for image-guided cancer surgery. Clin Transl Imaging. 2016;4:353-366.

44. Robertson R, Germanos MS, Li C, Mitchell GS, et al. Optical imaging of Cerenkov light generation from positron-emitting radiotracers. Phys Med Biol. 2009;54:N355-365.

45. Gill RK, Mitchell GS, Cherry SR. Computed Cerenkov luminescence yields for radionuclides used in biology and medicine. Phys Med Biol. 2015;60:4263-4280.

46. $\mathrm{Xu} \mathrm{Y}$, Liu H, Cheng Z. Harnessing the power of radionuclides for optical imaging: Cerenkov luminescence imaging. J Nucl Med. 2011;52:2009-2018.

47. Thorek DL, Robertson R, Bacchus WA, et al. Cerenkov imaging - a new modality for molecular imaging. Am J Nucl Med Mol Imaging. 2012;2:163-73.

48. Spinelli AE, Boschi F. Novel biomedical applications of Cerenkov radiation and radioluminescence imaging. Phys Medica. 2015;31:120-129.

49. Tanha K, Pashazadeh AM, Pogue BW. Review of biomedical Čerenkov luminescence imaging applications. Biomed Opt Express. 2015;6: 3053-3065.

50. Grootendorst MR, Cariati M, Pinder SE, et al. Intraoperative Assessment of Tumor Resection Margins in Breast-Conserving Surgery Using ${ }^{18} \mathrm{~F}-\mathrm{FDG}$ Cerenkov Luminescence Imaging: A Firstin-Human Feasibility Study. J Nucl Med. 2017;58:891-898. 



\section{PART I}

\section{REQUIREMENTS FOR INTRAOPERATIVE MARGIN ASSESSMENT IN PROSTATE CANCER SURGERY}





\section{CHAPTER 2}

STATE-OF-THE-ART INTRAOPERATIVE IMAGING TECHNOLOGIES FOR PROSTATE MARGIN ASSESSMENT:

ASYSTEMATIC REVIEW

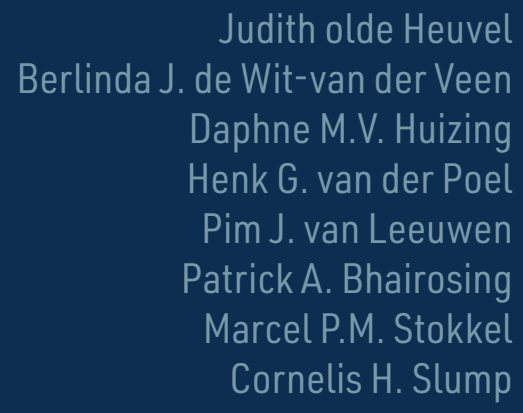

European Urology Focus 2020 [e-pub ahead of print] 


\section{Abstract}

\section{INTRODUCTION}

The main challenge in radical prostatectomy is complete excision of malignant tissue, while preserving continence and erectile function. Positive surgical margins (PSM) occur in up to $38 \%$ of cases, are associated with tumour recurrences, and may result in debilitating additional therapies. Despite surgical developments for prostate cancer ( $\mathrm{PCa}$ ), no technology is yet implemented to assess surgical margins of the entire prostatic surface intraoperatively. The aim of this systematic review is to provide an overview of novel imaging methods developed for intraoperative margin assessment in PCa surgery which are compared to standard postoperative histopathology.

\section{Methods}

A literature search of the last 10 years was conducted in the Scopus, PubMed and Embase (Ovid) databases. Eligible articles had to report the PSM rate according to their intraoperative margin assessment technology in comparison to standard 26 histopathology.

\section{Results}

The search resulted in 616 original articles, of which 11 articles were included for full-text review. The main technical developments in PCa margin assessment included Optical Coherence Tomography, Photodynamic Diagnosis with 5-Aminolevulinic Acid, Spectroscopy, and Enhanced Microscopy. These techniques are described and their main advantages, limitations, and applications in the clinical setting are discussed.

\section{CONCLUSION}

Several imaging methods are suggested in literature for detection of PSM during PCa surgery. Despite promising qualifications of the mentioned technologies, many struggle to find implementation in the clinic. Surgical conditions hampering the signal, long imaging times and comparison with histopathology are mutual challenges. The next step towards reduction of PSM in PCa surgery includes evaluation of these technologies in large clinical trials. 


\section{Introduction}

The main objective of radical prostatectomy (RP) is to ensure complete tumour resection while minimizing nerve, bladder and membranous urethra damage. Successful prostate carcinoma (PCa) surgery is established by both securing good therapeutic outcome and preserving sexual function and continence, the so-called trifecta [1]. However, incomplete tumour resections or positive surgical margins (PSM), defined as tumour on ink in histopathology, are observed in up to $38 \%$ of cases [2-4]. The risk on a PSM is higher with a higher T-category and biopsy Gleason score (GS). A PSM correlates with a shorter time to progression and increased rate of biochemical recurrence (BCR). Subsequently, complementary therapies such as androgen deprivation therapy or radiotherapy might be necessary for these males [5-7]. The presence of a PSM is associated with high preoperative PSA levels and high GS, pathological T-category and the surgeon's experience. These parameters only predict the chance of a PSM preoperatively, however the surgeon is not guided during the surgery to reduce the number of PSM observed post-surgery. The PSM rate is reduced when experienced surgeons perform RP, however, it is still present in up to $17 \%$ of the cases [8].

In current practise, the intraoperative frozen section (IFS) technique is available for margin assessment of suspected areas. After resection, the specimen is immediately frozen and stained, and areas of interest are evaluated for the presence of cancer cells. However, being a time-consuming procedure with low sensitivity (42\%), the clinical use of IFS is controversial $[9,10]$. Recently, an approach with improved sensitivity called Neurovascular Structure-Adjacent Frozen-section Examination (NeuroSAFE) was introduced to enable assessment of neurovascular structures adjacent to the prostate $[11,12]$. NeuroSAFE enables nerve sparing surgery in a larger number of patients with $93.5 \%$ sensitivity and $98.8 \%$ specificity compared to standard histopathology. The study of Schlomm et al. showed that patients with NeuroSAFE-evaluation had less post-surgical histopathological confirmed PSM compared to patients without NeuroSAFE (15.2\% vs. $21.7 \%)$, however no difference in BCR percentage was found [11]. A disadvantage of this technique is the requirement for standby qualified pathological personnel and the absence of the entire prostate circumference assessment. Although the examination time has a duration of at least 35 minutes, there is no prolonged surgical time in case of lymph node dissection following prostate removal [13]. 
There are several conditions to which imaging technologies should adhere in order to be used for intraoperative assessment in clinical practice. Ideally, margins are evaluated in vivo which obliges incorporation in minimally invasive surgery tools. Other requirements for margin assessment tools include fast, preferably real-time, examination times (order of minutes) without complicated sample preparation to minimize surgical delay. Next, the entire surface of the prostate specimen $(3-5 \mathrm{~cm}$ diameter) should be assessed to evaluate the total margin status and distance of tumour cells to the nerves. Furthermore, a high detection efficiency, sensitivity for micro metastases and a tumour to non-tumour distinction is mandatory without suffering from changes in tissue due to surgical intervention (i.e. coagulation) $[5,14,15]$. Finally, the technology should be user friendly, safe for both personnel and patients, and the specimen should be left sufficiently intact to perform standard diagnostic histopathological examination.

The aim of this systematic review is to identify new technologies for intraoperative tumour margin assessment for PCa, and subsequently, to evaluate the performance of these technologies compared to standard postoperative histopathology in detecting PSM status. 


\section{Methods}

\section{EVIDENCE ACQUISITION}

This review was registered on PROSPERO under registration number CRD42019124616 [16]. The literature search was performed by an information specialist (PAB) in Scopus, PubMed and Embase (Ovid) databases, according to the Preferred Reporting Items for Systematic Review and Meta-analysis (PRISMA) statement [17]. Different associations of the following keywords were applied in abstract and or title: prostate cancer; prostatectomy; margin; intraoperative; technology; imaging. Studies published between 2008 and 2019 were included. If imaging modalities were not implemented within 10 years, we presume that technologies were not suitable enough to be implemented in the clinic.

Letters, commentaries, editorials, case reports, reviews and conference abstracts were excluded, even as non-English manuscripts. Initially, titles and abstracts were screened by two reviewers independently $(\mathrm{JoH}, \mathrm{DMVH})$ to select publications for full-text review. Disagreement was resolved in consensus or with help of an independent reviewer (BJdWvdV).

\section{SELECTION Of FulL-TEXT PUblications}

Articles identified based on the search strategy were assessed for relevance and scientific quality. The technologies should compare their findings with standard histopathology. The articles have to focus on surgical margin assessment in PCa with an aim for actual intraoperative application. Studies were excluded if the technology was not applied in a surgical setting, for example only referred to preoperative margin prediction. Articles should at least include (1) basic description of the intraoperative technology, (2) quantitative or qualitative description of the margin, and (3) comparison of margin status to histopathological examination. Additional publications could be added to this review based on cross-referencing. Two independent reviewers screened full texts for selection (JoH, DMVH).

\section{RISK OF BIAS ASSESSMENT}

The included publications were assessed for Risk of Bias (RoB) by 3 reviewers (JoH, DMVH, BJdWvdV) independently, using criteria of the Quality Assessment of Diagnostic Accuracy Studies (QUADAS-2) tool [18]. As proposed by the QUADAS guidelines, four items were scored on having a low, high, or unclear RoB. Patient selection, 
index test (i.e., new technology), and reference standard (i.e., histopathology) were also assessed in terms of applicability. Initial disagreement between reviewers was resolved by discussion and consensus. 


\section{Results}

\section{GENERAL FINDINGS}

An overview of the selection procedure is visualized in Figure 1. In total 616 records were reviewed, in which 35 full text analysis were performed and a total of 11 articles fulfilled all selection criteria. In the following four consecutive sections are described: Optical Coherence Tomography (OCT) $(n=1)$, Photodynamic Diagnosis (PDD) with 5-Aminolevulinic Acid (ALA) ( $n=6)$, Spectroscopy $(n=2)$, and Enhanced Microscopy $(n=2)$.

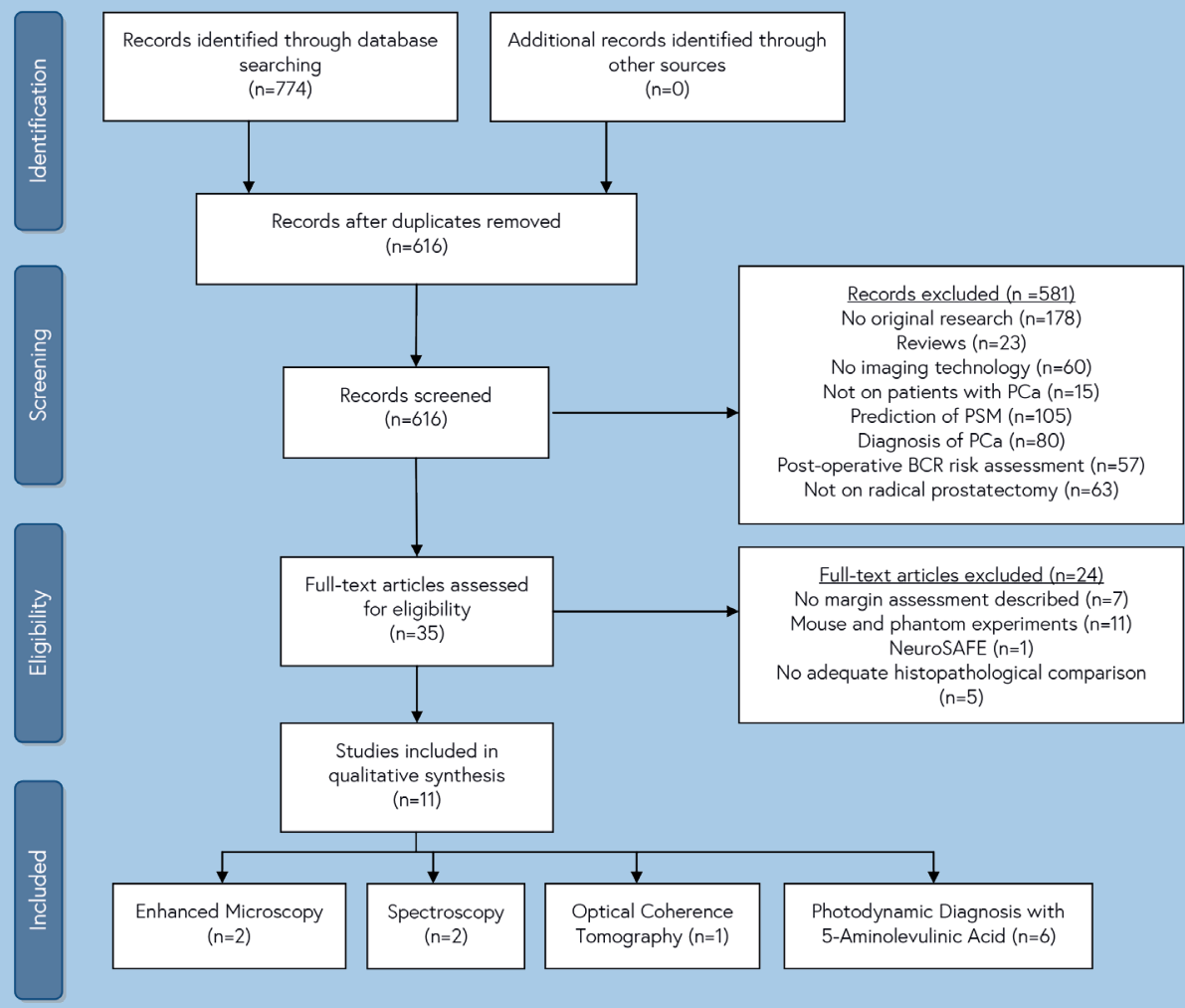

Figure 1. Selection workflow query, according to the PRISMA 2009 Flow Diagram [17]. PSM = Positive surgical margin, $\mathrm{PCa}=$ Prostate carcinoma

Table 1 shows the results of the Risk of Bias analysis performed with the QUADAS-2 tool of diagnostic studies. No study scored a low RoB on all seven items, whereas four publications had lower than five items with low RoB and applicability concerns. All studies had an unclear RoB considering reference standard, since either the ref- 
erence standard was performed with knowledge of the index test or no reference to the histopathological protocol was present. The RoB of the index test was unclear in some studies, due to an incomplete description of interpretation of the index test results. All included technologies were evaluated if requirements were met according to the previously stated criteria. Technical characteristics of all technologies are summarized in Table 2 and an overview of advantages and limitations is provided in Table 3.

\begin{tabular}{|c|c|c|c|c|c|c|c|}
\hline \multirow[b]{2}{*}{ Study } & \multicolumn{4}{|c|}{ Risk of bias } & \multicolumn{3}{|c|}{ Applicability concerns } \\
\hline & $\begin{array}{l}\text { Patient } \\
\text { selection }\end{array}$ & Index test & $\begin{array}{l}\text { Reference } \\
\text { standard }\end{array}$ & $\begin{array}{c}\text { Flow and } \\
\text { timing }\end{array}$ & $\begin{array}{l}\text { Patient } \\
\text { selection }\end{array}$ & Index test & $\begin{array}{l}\text { Reference } \\
\text { standard }\end{array}$ \\
\hline Dangle [19] & & & & & & & \\
\hline Ganzer [20] & & & & & & & \\
\hline Adam [21] & & & & & & & \\
\hline Inoue [22] & & & & & & & \\
\hline Fukuhara [2] & & & & & & & \\
\hline Fukuhara [2 & & & & & & & \\
\hline Zaak [25] & & & & & & & \\
\hline Lay [26] & & & & & & & \\
\hline Morgan [27] & & & & & & & \\
\hline Lopez [28] & & & & & & & \\
\hline Wang [29] & & & & & & & \\
\hline
\end{tabular}

Table 1. Risk of Bias assessment of the included studies obtained with the QUADAS-2 tool [18]. White = low risk, grey= unclear risk, black = high risk.

\section{OPTICAL COHERENCE TOMOGRAPHY}

OCT emits coherent light through tissue and the reflected light is used to reconstruct a cross-section of the imaged tissue. Light reflections are accordingly reconstructed in a 2D representation of the tissue architecture, a so-called tomogram [19]. OCT enables real-time evaluation of the entire prostatic circumference, but requires careful rinsing of the specimen to remove blood clots and non-prostatic tissue and manual rotation of the specimen. The analysis can be performed within 5 minutes with a commercially available CE-marked portable machine. OCT is able to assess extra prostatic extension and seminal vesicle invasion, in addition to margin evaluations [19]. Using OCT, Dangle et al. observed 21 out of 100 evaluated speci- 
mens with PSM and comparison with standard histopathology showed seven true positive and 14 false positive measurements (sensitivity 70\%, specificity 84\%) [19].

\section{Photodynamic Diagnosis WITH 5-AminOleVUliniC ACID}

5-ALA is a natural amino acid which is converted to photoactive protoporphyrin IX (PpIX) within the cell. 5-ALA shows higher accumulation in tumorous tissue compared to healthy tissue and is orally administered three to four hours prior to surgery for adequate uptake [20-22]. PpIX emits red fluorescence light after excitation with blue light, which can be detected using a commercially available laparoscopic compatible photodynamic camera. 5-ALA was evaluated in different small sample size studies (6-52 patients) and compared to histopathology [20-25]. These studies showed sensitivities ranging from 75\%-82\% and specificities between 68\%-88\%. The light source can be switched from white to blue light during surgery to excite PpIX, thereby not delaying the operating time [23]. Next, the 5-ALA PDD camera has the same view as the robotic camera, enabling real-time cancer location visualization in vivo.

\section{Light Reflectance Spectroscopy}

Spectroscopy visualizes the interaction between matter and electromagnetic radiation. Healthy and tumorous tissue are characterized by different tissue properties, regarding nuclear sizes and cell density [26], enabling tissue discrimination. Light reflectance spectroscopy (LRS) measures the intensity and spectrum of reflected or back scattered light. Using LRS, 17 specimens were analysed in the study of Morgan et al. and PSM were observed with $86 \%$ sensitivity and $85 \%$ specificity [27]. Lay et al. performed LRS measurements on 50 specimens, of which 197 areas were analysed. LRS was able to detect PSM in tumours with GS $\geq 7$ with $91 \%$ sensitivity and $93 \%$ specificity, in contrast to $65 \%$ and $88 \%$ in specimens with GS 6, respectively [26].

\section{CONFOCAL LASER ENDOMICROSCOPY}

Confocal laser endomicroscopy (CLE) is an endoscopic imaging tool based on standard confocal microscopy. The light beam penetrates at a specific depth at a certain time, from the multiple 2D images obtained, a 3D reconstruction of the specimen can be created. In the study of Lopez et al. a $488 \mathrm{~nm}$ laser was used for prostate imaging, in concurrence with a FDA approved fluorophore (sodium fluorescein) intravenously injected 20 minutes before CLE. Sensitivity and specificity were not 
mentioned in the study, and no PSM was found [28].

\section{Structured IlLUMination Microscopy}

Structured Illumination Microscopy (SIM) is an optical sectioning technique using a wide field illumination. In the study by Wang et al. a video-rate SIM (VR-SIM) is used to record images of the entire circumference in 24 patients. Each tissue frame is stitched together, to get a circumferential image of the entire prostate specimen. VR-SIM was able to detect a PSM in three of the four histologically confirmed cases, which had a size of $>1 \mathrm{~mm}$. In one case, VR-SIM detected a PSM which was not confirmed by histopathology and one PSM was missed by VR-SIM [29]. Sensitivity and specificity were not specified in the article, however calculation using the published data resulted in $75 \%$ sensitivity and $94 \%$ specificity. 
Please rotate the book

for the optimal experience

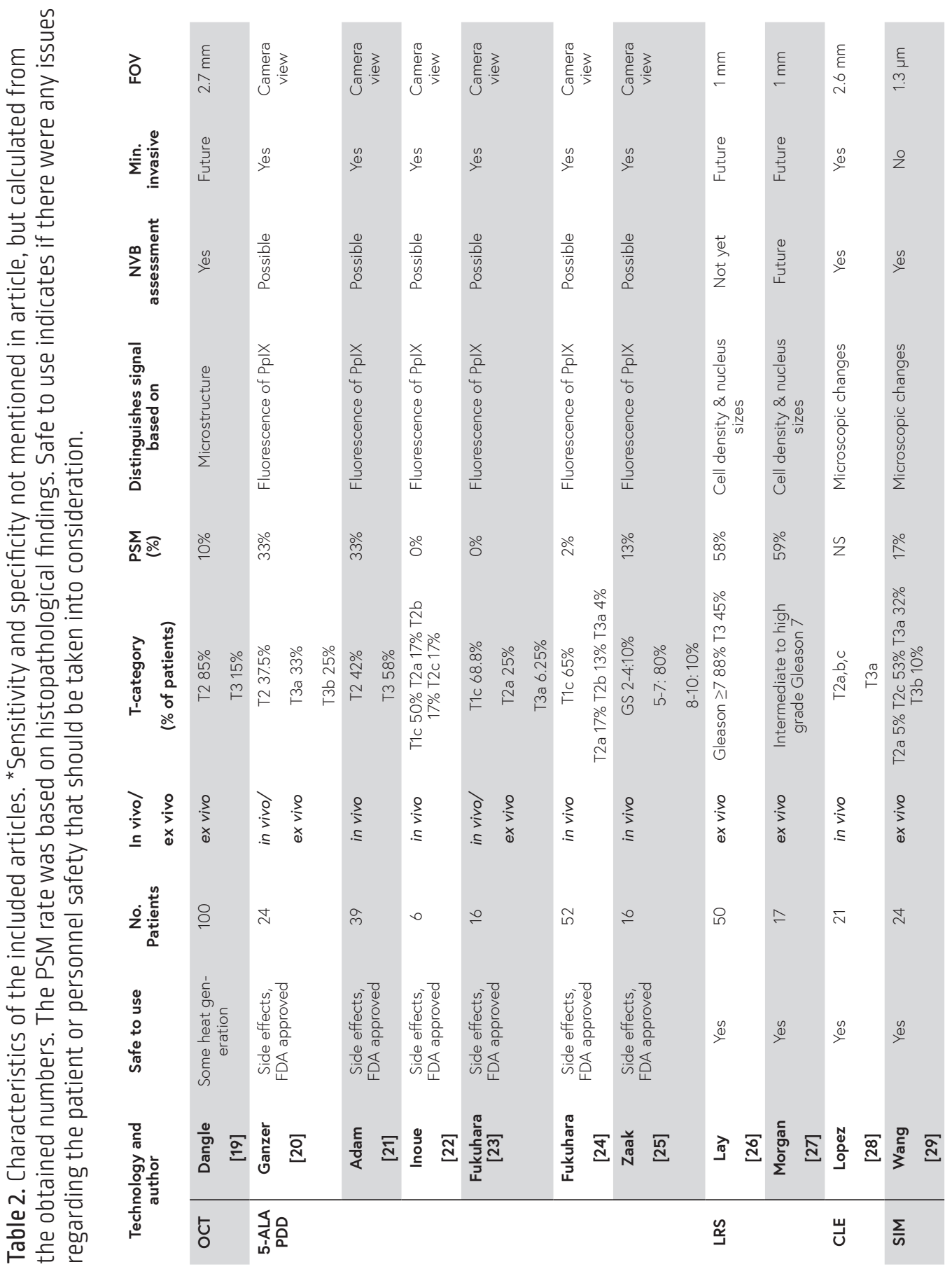




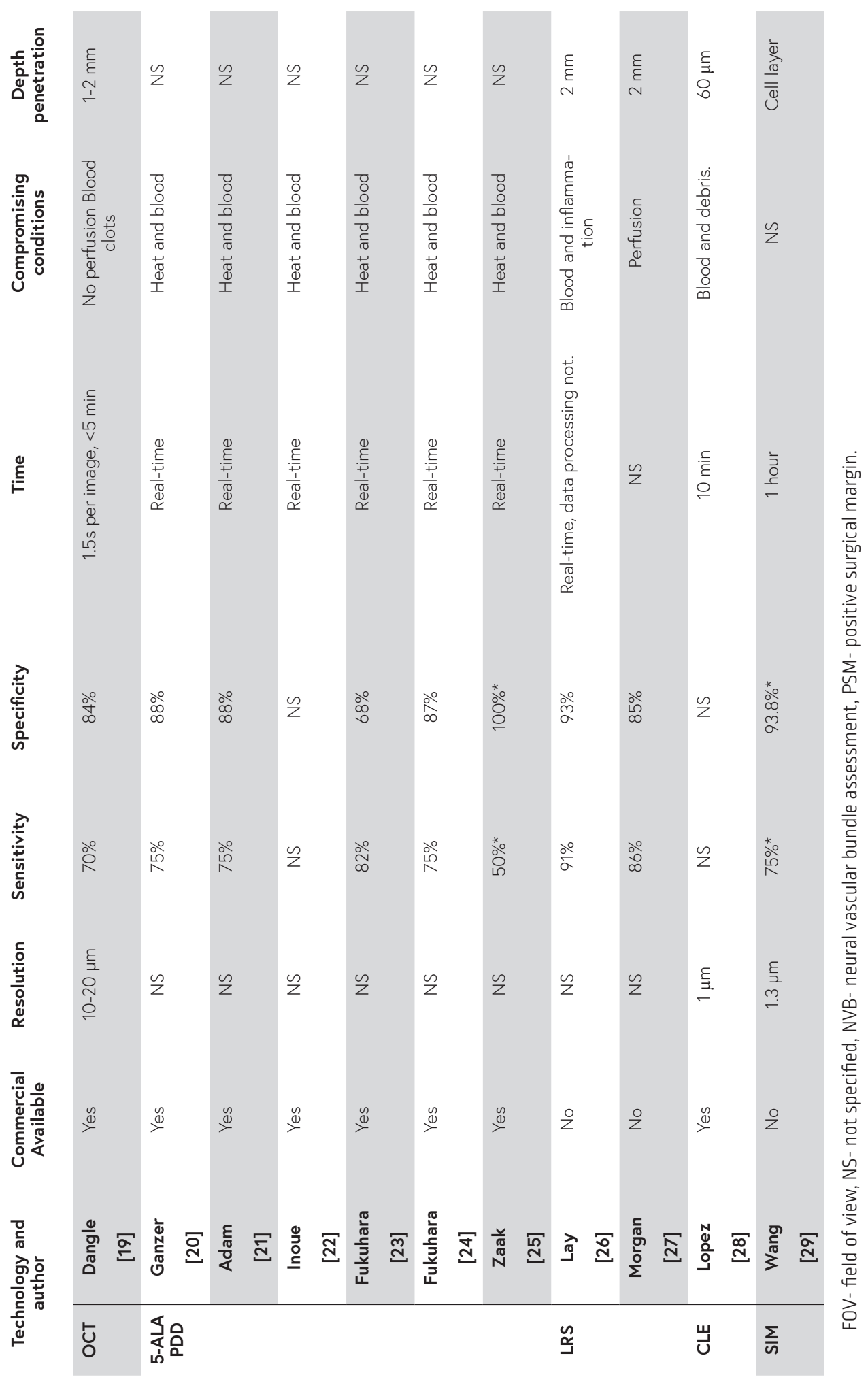




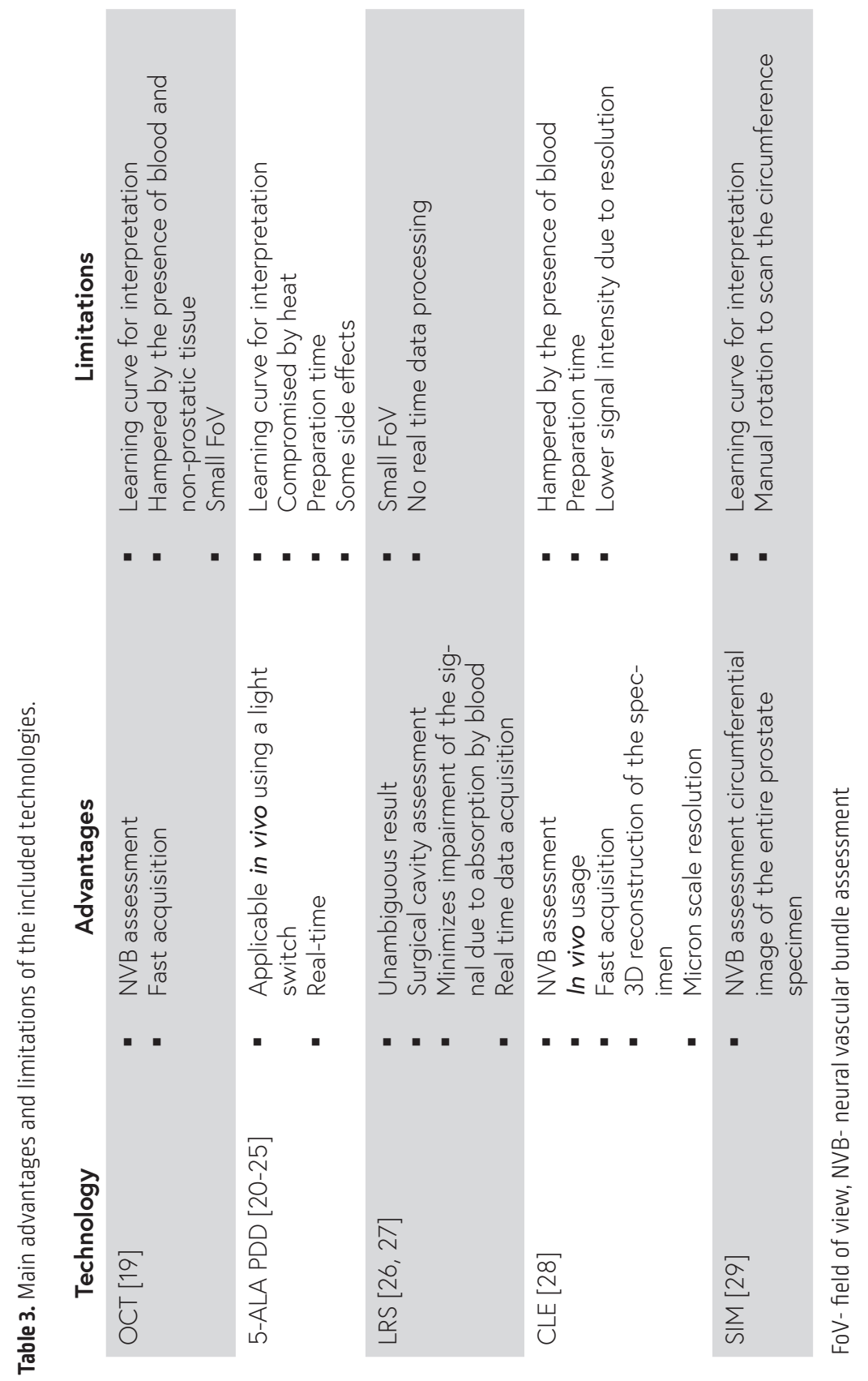




\section{Discussion}

Intraoperative margin assessment contributes to reduction of positive surgical margins in PCa surgery. This systematic review provides an overview of current possibilities for intraoperative margin assessment, including 5 different technologies. All included technologies are based on optical imaging of cellular differences between cancerous and normal tissue. Technologies like OCT and LRS seem promising, although still face drawbacks before clinical implementation is possible. So far, none of these techniques affect clinical decisions as they are purely used in research situations.

A Risk of Bias assessment of all selected studies was performed with the QUADAS-2 tool. A few points of consideration were extracted from this assessment. First observation was the general lack of studies with a methodology to answer our research question. Since most studies were still in the feasibility phase, they failed to compare their results properly to standard histopathology. Second, several studies performed their reference test (i.e., histopathology) not blinded for the results of the index test (i.e., new technology). This may result in a risk of bias regarding the 38 sensitivity and specificity, since it is possible that assessors alter histopathology results based on the technology's suggestions. On the other hand, marking suspicious locations based on the technology results enabled a direct comparison with histopathology in that specific location.

Previous reviews on technologies for surgical margin assessment in other tumour types predicted in 2014 an increase in the clinical use of these technologies [30-33]. Currently, only NeuroSAFE is embedded for PCa in daily practice in specific institutions, and one could ask why these technologies are not incorporated on a more frequent basis. This might be directly related to the strict histopathological definition of PSM in PCa, which is 'tumour on ink'. Hence, to be as precise as histopathology, a technology should assess margins with a depth resolution of one cell layer, which is challenging for the described intraoperative imaging technologies. In general, the sensitivity and specificity of the included technologies are inferior to the $93.5 \%$ sensitivity and $98.8 \%$ specificity of NeuroSAFE. However, the technologies included in this review are evaluated in limited sample sizes (range 6-100 patients) and are still under development. Next to that, with the introduction of new techniques a learning curve is common and could result in suboptimal detection rates. Still, the results were promising with specificities above 84\% (except for Fukuhara et al. [23]) 
and sensitivity ranging from 50-91\%.

Real-time tumour visualisation during PCa surgery may improve patient outcome and survival by assisting towards a more radical resection, while preserving vital normal tissues. The trifecta of surgical outcome are cancer control, sexual function and presence of continence. The first two can be evaluated if intraoperative margin assessment was combined with nerve sparing surgery, for example using the OCT, CLE, and SIM techniques to assess the neural vascular bundle. Furthermore, easy incorporation in clinical routine requires a user-friendly technology and an unambiguous interpretation of the results. A learning curve was required to distinguish OCT signals between tumour and normal tissue due to variations in prostate anatomy. This applies for 5-ALA as well, where training is required for the subjective interpretation of 5-ALA PDD [20,21]. In contrast, since spectroscopy technologies are based on computer-based algorithms, the conclusion will not primarily depend on user interpretation $[26,27]$.

Most included studies, except for the 5-ALA PDD and CLE studies, are currently only performed ex vivo yet all hold potential for in vivo usability. CLE images were acquired in vivo, though image analysis and interpretation were performed afterwards [28]. A general obstacle for in vivo usage is the influence of the surgical conditions on image acquisition. For example, the 5-ALA signal is compromised by heat $[21,23,24]$. Thus, the use of diathermy should be avoided is critical areas. To overcome this effect, the specimen can be prepared using a cold knife without electric devices [23]. Besides that, the signal of multiple technologies (5-ALA, OCT, CLE) is hampered by the presence of blood. Hence, careful rinsing of the prostate is required, complicating actual in vivo usage. However, research into different wavelengths which do not overlap with the heme-peak may overcome this problem. This could be performed for example using a specific wavelength range for LRS (700-850nm), which minimizes impairment of the signal due to absorption by blood $[26,27]$. Finally, OCT images can be altered by non-prostatic tissue through tissue interference and long periods without perfusion [19].

A shared disadvantage of all described techniques includes long assessment times, either due to long-lasting data processing or long acquisition times to assess the entire prostate with a small field of view (FoV). Spectroscopy, OCT, and microscopic techniques all have a FoV less than $5 \mathrm{~mm}$, therefore scanning the prostate circumference can take up to one hour. Full assessment of the prostate using a technology 
should be within 35 minutes to compete with intraoperative time constraints and to improve upon NeuroSAFE [11-13]. With future technical developments, the aforementioned technologies should be able to reduce assessment times. Preparation time needs to be considered when using fluorescent dyes with 5-ALA and CLE, as imaging should be performed 3-4 hours and within 20 minutes after administration, respectively [20-22,28]. Additionally, some side effects of 5-ALA have been reported [21], resulting in several contraindications for the oral use and restriction from sunlight after surgery to avoid skin reactions [20,23,24].

If a PSM is detected ex vivo by an optical technology, one of the problems is to map the PSM back to the surgical cavity to resect additional tissue. This mapping can be difficult, due to changes in the surgical field, thus a limiting factor for ex vivo imaging. Ideally, the technology would assess margins within the surgical cavity, for example using LRS which has the ability to measure the surgical cavity besides the excised specimen [26,27]. Based on all previous mentioned advantages and drawbacks, currently no technique is optimal for intraoperative PSM detection in PCa. Therefore, the search for alternative technologies persists and is likely to end-up in the use of fluorescent or radioactive markers to enhance signal intensities.

\section{Alternative AND FUtURe teCHNOLOGies}

This review focused on the clinical applicability of intraoperative margin assessment and several innovative technologies are developed for optical guidance during PCa surgery. Another strategy to decrease positive margins are surgical experience, preoperative selection, preoperative models and radio-guided surgery [34-36]. Investigations in the latter area are already ongoing, as well in combination with augmented and virtual reality [37,38]. Promising other techniques which are still in a pre-clinical development stage include fluorescence coupled to tumour targeted probes, like the prostate specific membrane antigen (PSMA). Tumour-targeting ligands to NIR fluorophores are already studied in (pre)-clinical trials in other cancer types. PSMA is often overexpressed by PCa cells and the ligand can be bound to an infrared fluorescent dye or Cy5 dye. This dye has absorption and emission wavelengths in the NIR range and in the far red range, enabling fluorescence imaging using a fluorophore [39-41]. PSMA can also be coupled to gallium-68 $\left({ }^{68} \mathrm{Ga}\right)$, enabling Cerenkov Luminescence Imaging during surgery using positron emitting properties of ${ }^{68} \mathrm{Ga}$. This technique has shown promising results in a pre-clinical setting [41]. 
In conclusion, several technologies are suggested to overcome the problem of post-surgery PSM in PCa. Despite promising specifications of the technologies mentioned, many struggle to find implementation in the clinic. Surgical conditions hampering the signal, long acquisition times and accurate comparison with histopathology are mutual challenges. Furthermore, large clinical trials are needed to investigate the added value of each technology in terms of improved patient outcome and cost-effectiveness, before incorporating margin assessment into clinical practice. Finally, improvements of the techniques are required to enhance embedding of intraoperative assessment of surgical margins. 


\section{REFERENCES}

1. Bianco FJ, Scardino PT, Eastham JA. Radical prostatectomy: Long-term cancer control and recovery of sexual and urinary function ("trifecta"). Urology. 2005;66:83-94

2. Samaratunga $H$, Montironi R, True L, et al. International Society of Urological Pathology (ISUP) Consensus Conference on Handling and Staging of Radical Prostatectomy Specimens. Working group 1: specimen handling. Mod Pathol. 2011;24:6-15

3. Izard JP, True LD, May P, et al. Prostate Cancer That Is Within $0.1 \mathrm{~mm}$ of the Surgical Margin of a Radical Prostatectomy Predicts Greater Likelihood of Recurrence. Am J Surg Pathol. 2014;38:333-338.

4. Stephenson AJ, Wood DP, Kattan MW, et al. Location, extent and number of positive surgical margins do not improve accuracy of predicting prostate cancer recurrence after radical prostatectomy. J Urol. 2009;182:1357-1363.

5. Ohori M, Wheeler TM, Kattan MW, et al. Prognostic Significance of Positive Surgical Margins in Radical Prostatectomy Specimens. J Urol. 1995;154:1818-24.

6. Silberstein J, Eastham J. Significance and management of positive surgical margins at the time of radical prostatectomy. Indian J Urol. 2014;30:423-428.

7. Bolla M, van Poppel H, Tombal B, et al. Postoperative radiotherapy after radical prostatectomy for high-risk prostate cancer: long-term results of a randomised controlled trial (EORTC trial 22911). Lancet. 2012;380:2018-2027.

8. Thompson JE, Egger S, Böhm M, et al. Superior Biochemical Recurrence and Long-term Quality-of-life Outcomes Are Achievable with Robotic Radical Prostatectomy After a Long Learning Curve-Updated Analysis of a Prospective Single-surgeon Cohort of 2206 Consecutive Cases. Eur Urol. 2018;73:664-671.

9. Goharderakhshan RZ, Sudilovsky D, Carroll LA, et al. Utility of intraoperative frozen section analysis of surgical margins in region of neurovascular bundles at radical prostatectomy. Urology. 2002;59:709-714.

10. Tsuboi T, Ohori M, Kuroiwa K, et al. Is intraoperative frozen section analysis an efficient way to reduce positive surgical margins? Urology. 2005;66:1287-1291.

11. Schlomm T, Tennstedt P, Huxhold C, et al. Neurovascular structure-adjacent frozen-section examination (NeuroSAFE) increases nerve-sparing frequency and reduces positive surgical margins in open and robot-assisted laparoscopic radical prostatectomy: experience after 11,069 consecutive patients. Eur Urol. 2012;62:333-340.

12. Mirmilstein G, Rai BP, Gbolahan $\mathrm{O}$, et al. The neurovascular structure-adjacent frozen-section examination (NeuroSAFE) approach to nerve sparing in robot-assisted laparoscopic radical prostatectomy in a British setting - a prospective observational comparative study. BJU Int. 2018;121:854-862.

13. Beyer B, Schlomm T, Tennstedt P, et al. A feasible and time-efficient adaptation of NeuroSAFE for da Vinci robot-assisted radical prostatectomy. Eur Urol. 2014;66:138-144.

14. Ackerman DA, Barry JM, Wicklund RA, et al. Analysis of risk factors associated with prostate cancer extension to the surgical margin and pelvic node metastasis at radical prostatectomy. J Urol. 1993;150:1845-1850.

15. Wieder JA, Soloway MS. Incidence, etiology, location, prevention and treatment of positive surgical margins after radical prostatectomy for prostate cancer. J Urol. 1998;160:299-315.

16. olde Heuvel J, Huizing DMV, de Wit - van der Veen BJ, et al. State-of-the-art intraoperative imaging technologies for prostate margin assessment: a systematic review. 2019 PROSPERO 
CRD42019124616.

17. Moher D, Liberati A, Tetzlaff J, et al. Preferred reporting items for systematic reviews and meta-analyses: the PRISMA statement. Ann Intern Med. 2009;151:264-269.

18. Whiting PF. QUADAS-2: A Revised Tool for the Quality Assessment of Diagnostic Accuracy Studies. Ann Intern Med. 2011;155: 529-536.

19. Dangle PP, Shah KK, Kaffenberger B, et al. The use of high resolution optical coherence tomography to evaluate robotic radical prostatectomy specimens. Int Braz J Urol. 2009;35:344-353.

20. Ganzer R, Blana A, Denzinger S, et al. Intraoperative photodynamic evaluation of surgical margins during endoscopic extraperitoneal radical prostatectomy with the use of 5-aminolevulinic acid. J Endourol. 2009;23:1387-1394.

21. Adam C, Salomon G, Walther S, et al. Photodynamic diagnosis using 5-aminolevulinic acid for the detection of positive surgical margins during radical prostatectomy in patients with carcinoma of the prostate: a multicentre, prospective, phase 2 trial of a diagnostic procedure. Eur Urol. 2009;55:1281-1288.

22. Inoue K, Ashida S, Fukuhara H, et al. Application of 5-aminolevulinic acid-mediated photodynamic diagnosis to robot-assisted laparoscopic radical prostatectomy. Urology. 2013;82:11751178.

23. Fukuhara $\mathrm{H}$, Inoue K, Satake $\mathrm{H}$, et al. Photodynamic diagnosis of positive margin during radical prostatectomy: Preliminary experience with 5-aminolevulinic acid. Int J Urol. 2011;18:585-591.

24. Fukuhara H, Inoue K, Kurabayashi A, et al. Performance of 5-aminolevulinic-acid-based photodynamic diagnosis for radical prostatectomy. BMC Urol. 2015;15:78.

25. Zaak D, Sroka R, Khoder W, et al. Photodynamic diagnosis of prostate cancer using 5-aminolevulinic acid--first clinical experiences. Urolog.y 2008;72:345-348.

26. Lay AH, Wang X, Morgan MS, et al. Detecting positive surgical margins: utilisation of light-reflectance spectroscopy on ex vivo prostate specimens. BJU Int. 2016;118:885-889.

27. Morgan MSC, Lay AH, Wang X, et al. Light Reflectance Spectroscopy to Detect Positive Surgical Margins on Prostate Cancer Specimens. J Urol. 2016;195:479-484.

28. Lopez A, Zlatev D V, Mach KE, et al. Intraoperative Optical Biopsy during Robotic Assisted Radical Prostatectomy Using Confocal Endomicroscopy. J Urol. 2016;195:1110-1117.

29. Wang M, Tulman DB, Sholl AB, et al. Gigapixel surface imaging of radical prostatectomy specimens for comprehensive detection of cancer-positive surgical margins using structured illumination microscopy. Sci Rep. 2016;6:27419.

30. Visgauss JD, Eward WC, Brigman BE. Innovations in Intraoperative Tumor Visualization. Orthop Clin North Am. 2016;47:253-264.

31. Keereweer $S$, Kerrebijn JDF, van Driel PBAA, et al. Optical Image-guided Surgery-Where Do We Stand? Mol Imaging Biol. 2011;13:199-207.

32. De Boer E, Harlaar NJ, Taruttis A, et al. Optical innovations in surgery. Br J Surg. 2015;102:5672.

33. Hsu M, Gupta M, Su LM, et al. Intraoperative Optical Imaging and Tissue Interrogation During Urologic Surgery. Curr Opin Urol. 2014;345:455-459.

34. Mohareri $\mathrm{O}$, Ischia J, Black PC, et al. Intraoperative Registered Transrectal Ultrasound Guidance for Robot-Assisted Laparoscopic Radical Prostatectomy. J Urol. 2015;193:302-312.

35. Ukimura $\mathrm{O}$, Aron M, Nakamoto $M$, et al. Three-Dimensional Surgical Navigation Model with 
TilePro Display During Robot-Assisted Radical Prostatectomy. J Endourol. 2014;28:625-630.

36. Shoji S, Aron M, de Castro Abreu AL, et al. Intraoperative ultrasonography with a surgeon-manipulated microtransducer during robotic radical prostatectomy. Int J Urol. 2014;21:736-739.

37. Porpiglia F, Fiori C, Checcucci E, et al. Augmented Reality Robot-assisted Radical Prostatectomy: Preliminary Experience. Urology. 2018;115:184.

38 Mehralivand S, Kolagunda A, Hammerich K, et al. A multiparametric magnetic resonance imaging-based virtual reality surgical navigation tool for robotic-assisted radical prostatectomy. Turkish J Urol. 2019;45:357-365.

39. Tringale KR, Pang J, Nguyen QT. Image-guided surgery in cancer: A strategy to reduce incidence of positive surgical margins. WIREs Syst Biol Med. 2018;10:1-18.

40. Kularatne SA, Thomas M, Myers CH, et al. Evaluation of Novel Prostate-Specific Membrane Antigen-Targeted Near-Infrared Imaging Agent for Fluorescence-Guided Surgery for Prostate Cancer. Clin Cancer Res. 2018;25:177-187

41. olde Heuvel J, de Wit - van der Veen BJ, Vyas KN, et al. Performance evaluation of Cerenkov Luminescence Imaging: A comparison of ${ }^{68} \mathrm{Ga}$ with ${ }^{18} \mathrm{~F}$. EJNMMI Phys. 2019;6:17 


\section{CHAPTER 3}

\section{PERFORMANCE EVALUATION OF CERENKOV LUMINESCENCE IMAGING:}

A COMPARISON OF ${ }^{68}$ GAWITH ${ }^{18} \mathrm{~F}$

Judith olde Heuvel

Berlinda J. de Wit-van der Veen

Kunal N. Vyas

David S. Tuch

Maarten R. Grootendorst

Marcel P.M. Stokkel

Cornelis H. Slump

EJNMMI Physics 2019;6:17 


\section{Abstract}

\section{INTRODUCTION}

Cerenkov luminescence imaging (CLI) is an emerging technology for intraoperative margin assessment. Previous research only evaluated radionuclide fluorine-18 $\left({ }^{18} \mathrm{~F}\right)$, however, for future applications in prostate cancer gallium-68 $\left({ }^{68} \mathrm{Ga}\right)$ seems more suitable, given its higher positron energy. Theoretical calculations predict that ${ }^{68} \mathrm{Ga}$ should offer a higher signal to noise ratio than ${ }^{18} \mathrm{~F}$; this is the first experimental confirmation. The aim of this study is to investigate the technical performance of CLI by comparing ${ }^{68} \mathrm{Ga}$ to ${ }^{18} \mathrm{~F}$.

\section{Methods}

The linearity of the system, detection limit, spatial resolution, and uniformity were determined with the LightPath imaging system. All experiments were conducted with clinically relevant activity levels in vitro, using dedicated phantoms.

\section{Results}

For both radionuclides, a linear relationship between the activity concentration and detected light yield was observed $\left(R^{2}=0.99\right) .{ }^{68} \mathrm{G}$ a showed approximately 22 times more detectable Cerenkov signal compared to ${ }^{18} \mathrm{~F}$. The detectable activity concentration after a 120 s exposure time and $2 \times 2$ binning of ${ }^{18} \mathrm{~F}$ was $23.7 \mathrm{kBq} / \mathrm{mL}$ and 1.2 $\mathrm{kBq} / \mathrm{mL}$ for ${ }^{68} \mathrm{Ga}$. The spatial resolution was $1.31 \mathrm{~mm}$ for ${ }^{18} \mathrm{~F}$ and $1.40 \mathrm{~mm}$ for ${ }^{68} \mathrm{Ga}$. The coefficient of variation of the uniformity phantom was 0.07 for the central field of view.

\section{CONCLUSION}

${ }^{68} \mathrm{Ga}$ was superior over ${ }^{18} \mathrm{~F}$ in terms of light yield and minimal detection limit. However, as could be expected the resolution was $0.1 \mathrm{~mm}$ less for ${ }^{68} \mathrm{Ga}$. Given the clinical constraints of an acquisition time less than 120 s and a spatial resolution $<2 \mathrm{~mm}, \mathrm{CLI}$ for intraoperative margin assessment using ${ }^{68} \mathrm{Ga}$ could be feasible. 


\section{Introduction}

In cancer surgery, the intraoperative distinction between malignant and healthy tissue is difficult. Incomplete surgical resection heavily impacts patient outcome because of the higher chance of additional therapy and worse prognosis [1-3]. A positive surgical margin (PSM), defined as tumour cells on the inked tissue margin on postoperative histopathology, increases the risk of disease recurrence. In radical prostatectomy it has been described that $11-38 \%$ of patients have a PSM [4-7].

An emerging optical technology in this respect is Cerenkov luminescence imaging (CLI) that visualizes the presence of $\beta$-emitting radionuclides by the detection of Cerenkov photons [8-11]. These photons originate when a $\beta$-particle travels faster than light in a specific dielectric medium [11-13]. The combination of nuclear and optical imaging has great potential for margin assessment as specific radiotracers are developed to target cancerous tissue and the Cerenkov radiation has a superficial penetration depth in tissue. Additionally, CLI has the advantage of using clinically approved imaging agents, which facilitates fast clinical translation of the technology. Academic researchers worldwide have already demonstrated the (pre) clinical feasibility of CLI for intraoperative specimen analysis [8,14-16]. The first clinical trial for margin assessment with CLI, performed in breast cancer surgery with fluorine-18-Fluorodeoxyglucose $\left({ }^{18} \mathrm{~F}-\mathrm{FDG}\right)$, has shown promising initial results [16].

To date, (pre-)clinical research in CLI has been mostly restricted to the metabolic radiotracer ${ }^{18} \mathrm{~F}-\mathrm{FDG}$ [11]. However, ${ }^{18} \mathrm{~F}-\mathrm{FDG}$ is not suitable as diagnostic imaging tool for prostate cancer, since prostate cancer is considered hypometabolic, and hence, accumulates only limited amounts of FDG $[17,18]$. Therefore, the more specific positron emitting radiotracer $\left[{ }^{68} \mathrm{Ga}\right] \mathrm{Ga}$-Prostate-Specific Membrane Antigen $\left({ }^{68} \mathrm{Ga}-\mathrm{PS}\right.$ MA-11) was introduced several years ago. PSMA is a transmembrane protein with significantly elevated expression on prostate cancer cells in comparison with benign prostatic tissue $[19,20]$. Furthermore, the isotope ${ }^{68} \mathrm{Ga}$ may be advantageous regarding Cerenkov light yield compared to ${ }^{18} \mathrm{~F}$ because of its higher initial positron energy. This results in more Cerenkov radiation from the positron, due to the energy content itself and the fact that more positrons will reach the Cerenkov threshold. This signal boost can be of importance, due to the generally low signal-to-noise ratio (SNR) of CLI [12]. The higher signal could reduce the injected activity and the acquisition time while reducing the imaging noise. According to Monte-Carlo simulations, ${ }^{68} \mathrm{Ga}$ can have theoretically a 26 times higher signal yield compared to ${ }^{18} \mathrm{~F}$ 
[21]. Nonetheless, it is known that these simulations may not comply with physical experiments $[12,21,22]$. Ciarrocchi et al. suggested as well, after in vitro studies concerning the performance of $\mathrm{CLI}$ using ${ }^{18} \mathrm{~F}$, to evaluate the impact of different PET radiotracers [23]. Thus, the aim of this research was to evaluate the technical performance of $\mathrm{CLI}$ using ${ }^{68} \mathrm{Ga}$, and to relate these outcomes to ${ }^{18} \mathrm{~F}$. Based on these study outcomes a CLI protocol that will fit clinical needs for margin assessment using ${ }^{68} \mathrm{Ga}$-PSMA-11 during prostate cancer surgery will be proposed. 


\section{Methods}

\section{EXPERIMENTAL DESIGN AND REQUIREMENTS}

The present study was designed to evaluate the performance of CLI comprising two relevant considerations; experiments should be conducted with clinically relevant activity levels and imaging protocols. Next to that, experiments were optimized with the following practical requirements: fast acquisition time, radiation safety, high sensitivity for small lesions and good tumour to non-tumour distinction. Radioisotopes used in this study were 2-Deoxy-2-(18F) Fluoroglucose (IBA Molecular) and ${ }^{68} \mathrm{Ga}$ coupled to Glu-urea-Lys (Ahx)-HBED-CC (Scintomics GmbH). The measured activity levels for ${ }^{68} \mathrm{Ga}$-PSMA-11 in prostate cancer were leading for all experiments, and ${ }^{18} \mathrm{~F}$ activity levels were adapted accordingly.

\section{TUMOUR UPTAKE ON PET SCANS}

The ${ }^{68} \mathrm{Ga}$-PSMA-11 concentration of 30 primary prostate cancer tumours and benign prostate tissue was measured on a Positron Emission Tomography/Computed Tomography (PET/CT) scan in order to conduct the in vitro experiments with clinically relevant radioactivity concentrations. Patients had undergone a PET/CT scan on a Philips Gemini TF system (Philips, Best, The Netherlands), 45 minutes after an intravenous injection of $\sim 100 \mathrm{MBq}{ }^{68} \mathrm{Ga}-\mathrm{PSMA}-11$. Acquisitions were performed from midthigh to skull base starting with 3 minutes per bed position in the pelvic area and 2 minutes per bed over the remaining body. PET images were reconstructed using BLOB-OS-TF without any post-reconstruction filter, including corrections for decay, random coincidences, dead time, low dose CT-based attenuation and scatter. The average tumour uptake in Becquerel $(\mathrm{Bq})$ per $\mathrm{mL}$ was measured using a spherical $\mathrm{VOI}$ placed around the tumour lesion, with a minimal size of $3.3 \mathrm{~cm}^{2}$ (Osirix MD DICOM viewer v.9.2, Pixmeo SARL, Bernex, Switzerland).

\section{Cerenkov Luminescence ImAGING SYSTEM AND ACQUISITION SETTINGS}

The LightPath optical imaging system (Lightpoint Medical Ltd, Chesham, United Kingdom) is developed for intraoperative margin assessment using the Cerenkov radiation induced by $\beta$-emitting radionuclides. This system is equipped with a camera lens $\left(F / 0.95,512 \times 512\right.$ pixels) coupled through optics to a $-80^{\circ} \mathrm{C}$ cooled electron multiplying charge coupled device (EMCCD, Andor iXon Ultra 897). The EMCCD is shielded with a tungsten plate and folded optics are used to reduce the number of gamma photons striking the EMCCD sensor. A standard optical camera (F/1.4 lens, 
$1600 \times 1200$ pixels) was used to acquire white light reference images. The system has a light tight imaging chamber to shield from ambient light.

In this study, images were initially acquired using the previously published protocol for ${ }^{18} \mathrm{~F}$ with an exposure time of 300s and 8-pixel binning (E300B8) [16]. To find a suitable protocol for clinical application using ${ }^{68} \mathrm{Ga}$, acquisition settings were varied with exposure times of 60,120, and 300s without or with the use of 2, 4, or 8-pixel binning. Acquisition protocols applied for both radionuclides. Unless otherwise specified, images were acquired without an optical filter. Data analysis was performed using MATLAB R2017b (The MathWorks, Natick, 2017).

\section{LINEARITY AND DETECTION LIMIT}

Three $2 \mathrm{~mL}$ Eppendorf tubes of both radionuclides were filled with 2.5, 12.5 and $45 \mathrm{kBq} / \mathrm{mL}$ diluted in $1 \mathrm{~mL}$ water to investigate the linearity of the system, i.e. to evaluate the correlation between the signal intensity and activity concentration. CLI imaging was performed every 20 minutes during four subsequent hours and images were processed with the LightPath software, by applying a median filter $(3 \times 3$ pixels) and Gaussian filter ( 3 pixels) to reduce the noise from high energy photons, also known as gamma strikes. The latter are $511 \mathrm{keV}$ annihilation photons emitted from the radionuclide, visualised as a local high signal spike with a characteristic tail. After filtering, CLI images were aligned to the white light reference image.

The radiance (photons $/ \mathrm{s} / \mathrm{cm}^{2} / \mathrm{sr}$ ) per activity concentration was used to compare the signal of both radionuclides. The average radiance was obtained by manually selecting a region of interest (ROI) of 150 pixels around the Cerenkov signal of the Eppendorf tubes, and a $\mathrm{ROI}$ outside the Eppendorf tubes to determine the average background signal. A possible linear relationship between radiance and activity concentration will be verified by comparing the radiance half-life $\left(R_{t 1 / 2}\right)$ to the decay half-life of both radionuclides. The radiance half-life is the time required to reduce the radiance to half of its initial value.

The radiance per activity concentration was used to determine the detection limit. The lowest detectable activity concentration was defined as the activity concentration where the SNR is 2, which should be sufficient to distinguish the signal in the tumour from the background [24]. The SNR represents difference between the mean signal and the mean background, divided by the standard deviation of the background. The detection limit was determined for both radionuclides and using 
multiple acquisition settings.

All of the described experiments were repeated under $\sim 1 \mathrm{~mm}$ raw chicken breast fillet, to simulate the influence of tissue on the signal attenuation and minimal detectable activity concentration. Raw chicken breast was stacked on top of three Eppendorf tubes containing the same activity concentrations as the ones described above.

\section{EFFECTIVE SPATIAL RESOLUTION}

The spatial resolution was determined using glass capillary tubes (outer diameter (OD) $1.1 \mathrm{~mm}$ ) filled with $33 \mathrm{kBq}{ }^{18} \mathrm{~F}$ and ${ }^{68} \mathrm{Ga}$. The effect of tissue on the spatial resolution was determined using $\sim 1 \mathrm{~mm}$ chicken breast fillet on top of the capillaries. The spatial resolution was obtained by averaging intensity profiles perpendicular through cross sections of the imaged line source on raw imaging data without filtering. The average full width half maximum (FWHM) was calculated over $40 \mathrm{~mm}$. Signal intensities were corrected for the zero background level, which was the level of digital counts in the image without radioactivity present. Thereafter, intensities were normalised to correct for possible difference in activity and maximum intensity of ${ }^{18} \mathrm{~F}$ and ${ }^{68} \mathrm{Ga}$.

\section{UNIFORMITY}

The uniformity of the field of view (FoV) was determined using a square uniform Perspex phantom $(60 \times 60 \mathrm{~mm})$ with $4.3 \mathrm{MBq}{ }^{68} \mathrm{Ga}$ diluted in $15 \mathrm{~mL}$ water. Three subsequent images were acquired using an exposure of 60 seconds without binning. Uniformity was determined on the unfiltered image and after filtering with a median and Gaussian. The median value of three subsequent CLI images were used for analysis, to reduce measurement uncertainty and the effect of the high energy gamma photons. Uniformity was measured by the mean and standard deviation of the signal by using a ROI over the entire useful field of view, UFoV and accordingly quantified using the coefficient of variation (CoV). The uniformity of the central field of view (CFoV), defined as $0.75 \times$ UFoV, was determined as well [25].

\section{SIGNAL-TO-BACKGROUND RATIO}

A signal-to-background ratio (SBR) experiment was conducted to assess the capability of $\mathrm{CLI}$ to distinguish tumour from background tissue. The mean ${ }^{68} \mathrm{Ga}-\mathrm{PSMA}-11$ concentrations $(\mathrm{kBq} / \mathrm{mL})$ of the tumour and the background, derived from the clinical PET scans, were diluted in an Agar solution (0.4 g agar powder in $20 \mathrm{~mL}$ of 
distilled water). Agar is a mixture of agarose and agaropectin, resulting in a gel like substance. From both solidified Agar solutions, a cube $(2 \times 2 \mathrm{~cm})$ was excised and exchanged with the other Petri dish (see Figure 3), and a SBR of 2:1 was obtained. The petri dish was subsequently imaged with different acquisition settings to verify the ability to distinguish the signal. An intensity profile was drawn over the cross section of the Petri dish, to obtain the digital counts per pixel over this line. 


\section{Results}

\section{TUMOUR UPTAKE ON PET SCANS}

The average ${ }^{68} \mathrm{Ga}$-PSMA-11 PET/CT uptake in prostate cancer tumours and in benign tissue is presented in Table 1, showing a large interpatient variation in tumour uptake, tumour volume, and tumour-to-background ratio (TBR). To fit the clinical needs, the $\mathrm{CLI}$ should at least detect an activity concentration of $1.59 \mathrm{kBq} / \mathrm{mL}$. In vitro experiments should be conducted with the clinical uptake range of $1.59-8.54 \mathrm{kBq} / \mathrm{mL}$.

Table 1. Overview of ${ }^{68} \mathrm{Ga}$-PSMA-11 tumour uptake on PET/CT. VOI-volume of interest

\begin{tabular}{c|cccc} 
& $\begin{array}{c}\text { Volume of } \\
\text { Interest } \\
(\mathbf{m L})\end{array}$ & $\begin{array}{c}\text { Mean Tumour } \\
\text { Uptake } \\
(\mathbf{k B q} / \mathbf{m L})\end{array}$ & $\begin{array}{c}\text { Mean Uptake } \\
\text { Tumour } \times \text { VOI }\end{array}$ & $\begin{array}{c}\text { Tumour to } \\
\text { Background Ratio }\end{array}$ \\
\hline Average & 17.4 & 3.35 & $\mathbf{( k B q )}$ & \\
\hline Standard deviation & 11.4 & 1.53 & 80.96 & 2.7 \\
\hline Minimum & 3.3 & 1.59 & 71.50 & 1.1 \\
\hline Maximum & 53.0 & 8.45 & 11.20 & 5.7 \\
\hline Median & 14.3 & 2.62 & 275.24 & 2.5 \\
\hline
\end{tabular}

\section{LINEARITY AND DETECTION LIMIT}

For both radionuclides, an excellent linear relationship between the radioactivity concentration and detected light yield (radiance) was observed $\left({ }^{18} \mathrm{~F}: \mathrm{R}^{2}=0.98\right.$; ${ }^{68} \mathrm{Ga}: R^{2}=0.99$ ) for Exposure 120 s and Binning 2 (E120B2), Figure 1. ${ }^{68} \mathrm{Ga}$ resulted in approximately 22 times more signal compared to ${ }^{18} \mathrm{~F}$ with similar imaging settings. CLI signal linearity within the clinical prostate cancer tumour uptake range on ${ }^{68} \mathrm{Ga}$-PSMA-11 PET/CT was for above $\mathrm{R}^{2}=0.95$, whereas ${ }^{18} \mathrm{~F}$ decreased to $\mathrm{R}^{2}=0.74$ and $R^{2}=0.42$, without and with tissue surrogate respectively (supplemental Figure 1 ). The linear relationship remained with the addition of tissue surrogate on the Eppendorf tubes ( $\left.{ }^{18} \mathrm{~F}: \mathrm{R}^{2}=0.95 ;{ }^{68} \mathrm{Ga}: \mathrm{R}^{2}=0.99\right)$, Figure 1. Although, the signal intensity decreased to $73 \%$ and $62 \%$ of the original ${ }^{18} \mathrm{~F}$ and ${ }^{68} \mathrm{Ga}$ signal, respectively. Acquisition with Exposure 300s and Binning 8 (E300B8) setting, resulted in a linear relationship of ${ }^{18} \mathrm{~F}: \mathrm{R}^{2}=0.97 / 0.95$ and ${ }^{68} \mathrm{Ga}: \mathrm{R}^{2}=0.97 / 0.93$ with and without tissue surrogate, respectively. The radiance half-life of CLI was attained in $115 \mathrm{~min}$ for ${ }^{18} \mathrm{~F}\left(\tau=0.006 \mathrm{~min}^{-1}\right.$; $\left.\mathrm{R}^{2}=0.98\right)$ and within $69 \mathrm{~min}$ for ${ }^{68} \mathrm{Ga}\left(\tau=0.01 \mathrm{~min}^{-1} ; \mathrm{R}^{2}=0.99\right)$, both approximating the radionuclide half-life times of 109 and 68 min, respectively. The detection limit for both radionuclides, with and without tissue surrogate can be found in Table 2 (See also supplemental Figure 2 for the visual representation of the detection limit). 

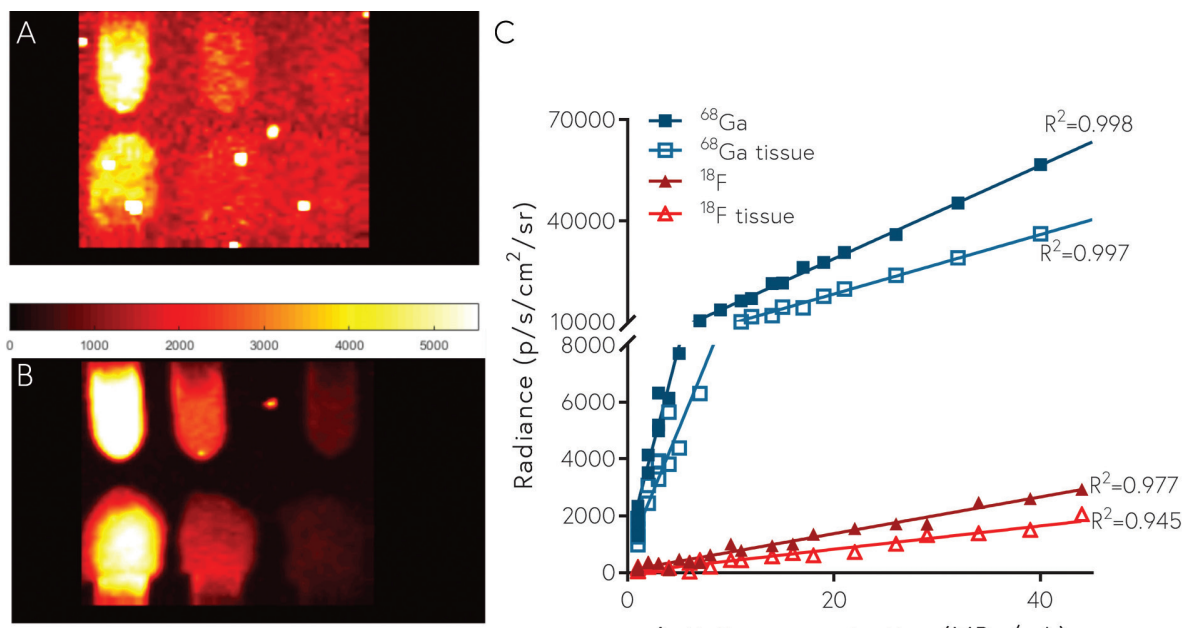

Activity concentration $(\mathrm{MBq} / \mathrm{mL})$

Figure 1. Example of two CLI images of 6 Eppendorf tubes filled with ${ }^{18} \mathrm{~F}[\mathrm{~A}]$ and ${ }^{68} \mathrm{Ga}[\mathrm{B}]$, where the bottom row is overlaid with $1 \mathrm{~mm}$ tissue [A]. From left to right the tubes are filled with $1 \mathrm{~mL}$ of $45,12.5$ and $2.5 \mathrm{kBq} / \mathrm{mL}$ activity. Effect of tissue on linearity and signal intensity ${ }^{18} \mathrm{~F}$ and ${ }^{68} \mathrm{Ga}$ [C]. Data acquired with an exposure time of 120 s and binning $2 \times 2$. ${ }^{68} \mathrm{G}$ a gives a 22 times higher light yield compared to ${ }^{18} \mathrm{~F}$ on average. The break line was added to the graph, to visualize the effect of tissue on the ${ }^{18} \mathrm{~F}$ radiance. Without break line, this was not visible, due to the high radiance of ${ }^{68} \mathrm{Ga}$. Please note, the bright dots not relative to the object are noise signal, also known as gamma strikes.

Table 2. Minimal detectable activity concentration $[\mathrm{kBq} / \mathrm{mL}]$ for ${ }^{18} \mathrm{~F}$ and ${ }^{68} \mathrm{Ga}$ with $\mathrm{SNR}=2$, using different acquisition protocols.

\begin{tabular}{|c|c|c|c|c|}
\hline \multirow[b]{2}{*}{ Acquisition } & \multicolumn{2}{|c|}{${ }^{18} \mathrm{~F}$} & \multicolumn{2}{|c|}{${ }^{68} \mathrm{Ga}$} \\
\hline & 300 s \& bin $8 \times 8$ & $120 s \&$ bin $2 \times 2$ & $300 s$ \& bin $8 \times 8$ & $120 s \&$ bin $2 \times 2$ \\
\hline No tissue & 3.42 & 23.66 & 0.14 & 1.18 \\
\hline Tissue & 5.04 & 39.16 & 0.24 & 1.78 \\
\hline
\end{tabular}

\section{EFFECTIVE SPATIAL RESOLUTION}

The FWHM at E120B2 and E300B8 was for ${ }^{18} \mathrm{~F} 1.31$ and $1.61 \mathrm{~mm}$, respectively and for ${ }^{68} \mathrm{Ga}: 1.40$ and $1.85 \mathrm{~mm}, 1 \mathrm{~mm}$ respectively (see Figure 2). The addition of $\sim 1 \mathrm{~mm}$ tissue surrogate increased the FWHM for both settings 2.40 and $2.85 \mathrm{~mm}$ for ${ }^{18} \mathrm{~F}$ and 2.73 and $3.40 \mathrm{~mm}$ for ${ }^{68} \mathrm{Ga}$, respectively. 

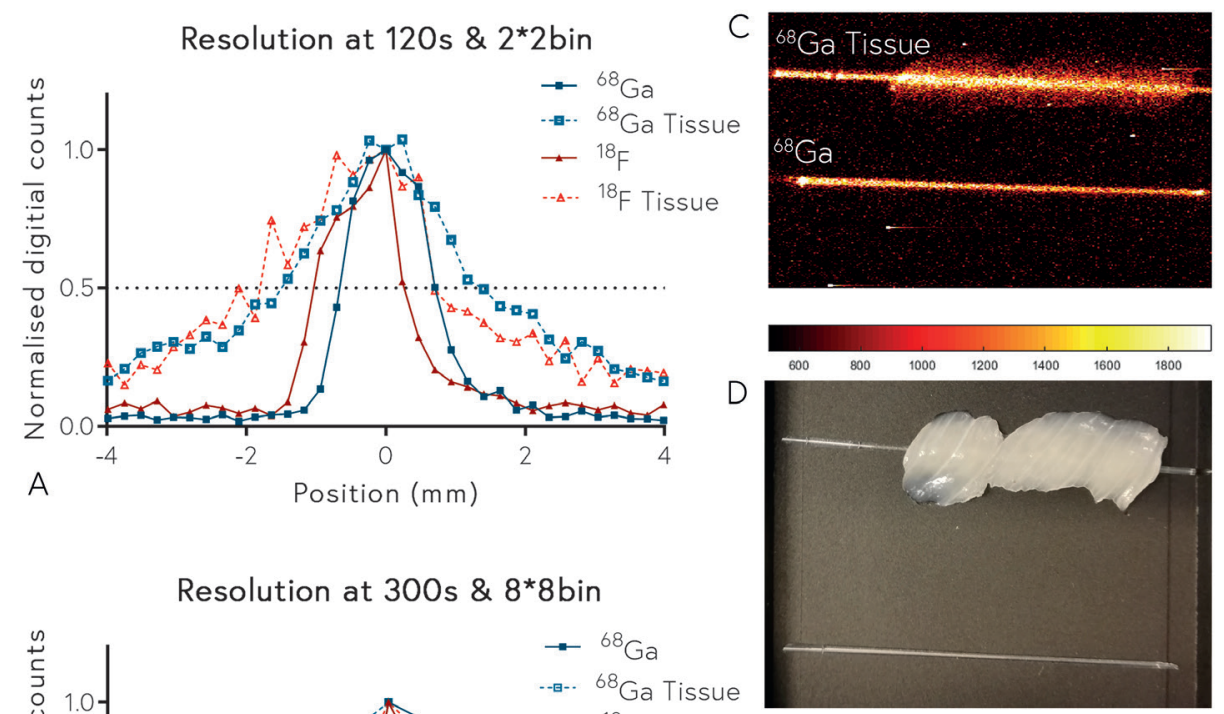

Resolution at 300 s $\& 8$ * 8 bin
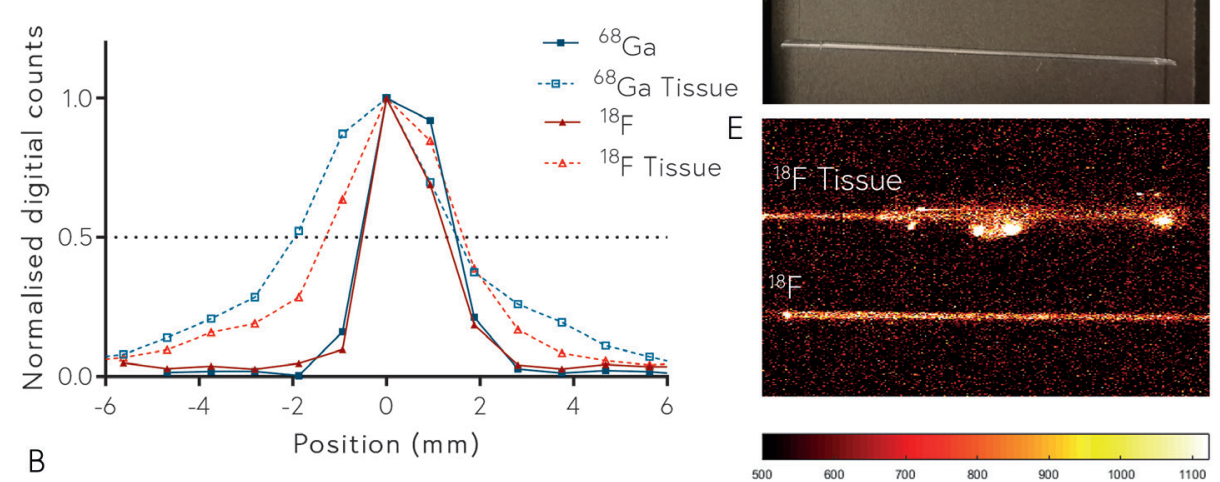

Figure 2. The FWHM of ${ }^{18} \mathrm{~F}$ (blue] and ${ }^{68} \mathrm{Ga}$ (red] for 120 s and $2 \times 2$ binning $[\mathrm{A}$ ), 300 s and $8 \times 8$ binning $[\mathrm{B}$ ), the influence of tissue on spatial resolution is displayed in light blue and light red. Signal intensities were normalised, to correct for the difference in activity and intensity of ${ }^{18} \mathrm{~F}$ and ${ }^{68} \mathrm{Ga}$. D shows a white light image of the glass capillary tubes with and without tissue. In C the corresponding CLI image of ${ }^{68} \mathrm{Ga}$ and in E the CLI images where the capillaries are filled with ${ }^{18} \mathrm{~F}$. The CLI images visualized were used as input to determine the FWHM over a line profile. The displayed images were acquired with 120 s and $2 \times 2$ binning. Please note, the bright dots not relative to the object are noise signal, also known as gamma strikes.

\section{UNIFORMITY}

The unfiltered median of three uniformity phantom CLI images showed a CoV of 0.18 of both the UFoV and CFoV. After Gaussian and median filtering, the uniformity of the images improved to a CoV of 0.09 in the UFoV and 0.07 in the CFoV (Supplemental Figure 3). 


\section{SIGNAL-TO-BACKGROUND RATIO}

Figure 3 shows the results of the signal-to-background experiment, where binning improved the SBR, yet compromised the sharpness of the transition from background to signal. Analysis of the intensity profiles showed a SBR of 2.1 using the E60B4 protocol, which is comparable to the activity concentration ratio of the tumour and background Agar solutions. E60B1 and E120B2 resulted in a SBR of 2.16

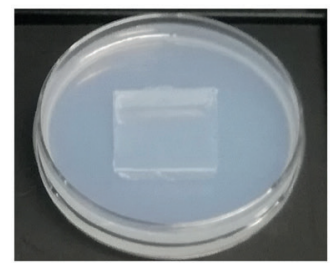

A

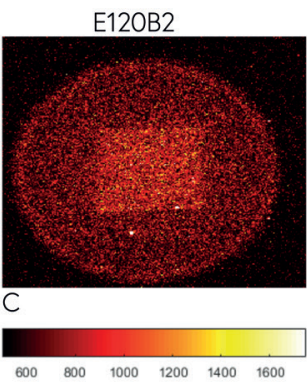

E60B4

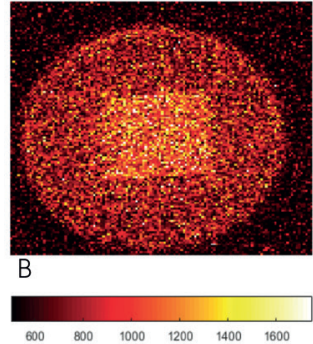

E60B1

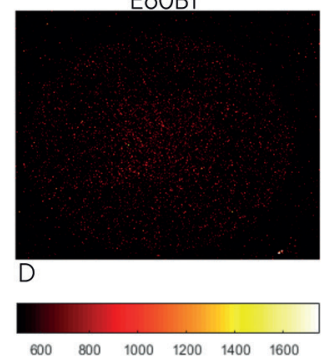

Signal to Background Ratio (2:1)

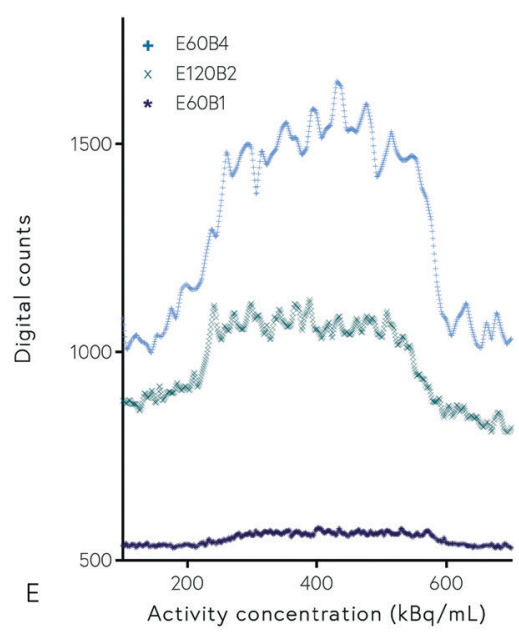

Activity concentration $(\mathrm{kBq} / \mathrm{mL})$

Figure 3. A representative image of the signal-to-background ratio experiment setup [A]. The result of three different exposure times and binning settings [B-D]. The graph of intensity profile across the previous three Petri dishes [E]. Please note, the bright dots not relative to the object are noise signal, also known as gamma strikes. 


\section{Discussion}

In this study the optical properties and performance of $\mathrm{CLI}$ using ${ }^{68} \mathrm{Ga}$ were compared to those of ${ }^{18} \mathrm{~F}$, under clinically relevant activity levels. The radiance was linearly associated with the activity of both radionuclides $\left({ }^{18} \mathrm{~F}: \mathrm{R}^{2}=0.98 ;{ }^{68} \mathrm{Ga}: \mathrm{R}^{2}=0.99\right)$. The linear relationship persisted with the addition of roughly $1 \mathrm{~mm}$ of the tissue surrogate. ${ }^{68} \mathrm{G}$ a showed a 22 times stronger radiance compared to ${ }^{18} \mathrm{~F}$ in the current study. Glaser et al. simulated the fluence rates with presence of scatter and absorption, to mimic optical properties of tissue, where a higher fluence rate was observed for ${ }^{68} \mathrm{Ga}$ [26]. Fan et al. and Beattie et al. compared fluence rates for ${ }^{18} \mathrm{~F}$ and ${ }^{68} \mathrm{Ga}$ measured with IVIS cameras, and found radiation rates of 15 and 19 times higher for ${ }^{68} \mathrm{Ga}[22,27]$. This signal intensity difference between ${ }^{18} \mathrm{~F}$ and ${ }^{68} \mathrm{G}$ a was slightly lower than the 26 times factor the Monte-Carlo simulations found in literature [21]. This inconsistency may be explained by certain definitions in Monte-Carlo experiments, such as the use of true point sources in MC-simulations, whereas the relatively larger volume of an Eppendorf tube will radiate from multiple angles onto the camera. Other effects may include machine differences and non-linear detector efficiencies of the different camera systems. Furthermore, in real-life experiments a fraction of positrons will escape from the medium before emitting Cerenkov photons [12]. Still, our results underline the superiority of ${ }^{68} \mathrm{Ga}$ for Cherenkov imaging with respect to signal yield.

Our results indicate that this specific CLI system was not homogeneous over the entire FoV, given the found CoV of 0.18 in the raw image. Visual inspection of the $\mathrm{CL}$ image shows a weaker signal near the edges of the uniformity phantom. Though this observation could be due to a commonly encountered phenomenon in optics known as lens vignetting, the results may also have been influenced by the relatively small size of the phantom which matches the $60 \times 60 \mathrm{~mm}$ FoV exactly. According$l y$, the edges of the phantom are visualised as 'darker' due to the lack of positron emissions from the material just outside the FoV. To account for this problem a uniformity phantom should cover the FoV with an additional margin on all sides that is greater than the positron range. The vignetting in the FoV does not hamper the clinical assessment, since the size of average prostate is small enough to fit in the CFoV [28]. Thus, it is suggested to only use the CFoV to image the specimen. The $\mathrm{CoV}$ of the processed image, which is part of the clinical CLI protocol, in the CFoV was 0.07 without binning. This was considered uniform enough to leave out addi- 
tional post-processing steps to improve the uniformity. The experiment was only performed with ${ }^{68} \mathrm{Ga}$, since uniformity of the system is expected to be independent of the radionuclide. However, the larger positron range could alter the texture of the image in comparison to a shorter range.

The resolution response was determined using a glass capillary with an outer diameter of $1.1 \mathrm{~mm}$. Though the camera is able to image up to $158 \mu \mathrm{m}$ according to the specifications, a better resolution is not deemed clinically relevant, as the surgeon is not able to resect with a higher accuracy. Still, we underline that difference in resolution between ${ }^{18} \mathrm{~F}$ and ${ }^{68} \mathrm{G}$ a found in this study is not entirely trivial, especially when the tissue surrogate was added. At higher binning (E300B8) this difference with tissue was roughly $0.5 \mathrm{~mm}$. If a PSM occurs, surgeons are only able to shave in a specific area surrounding the PSM. Though this shaving cannot be performed with a submillimetre precision, the localisation of the suspected area should be as precise as possible. That being said, a PSM found on ex vivo measurement is difficult to map back in vivo, therefore the lower resolution of ${ }^{68} \mathrm{Ga}$ is will not hamper clinical implementation. The smaller resolution of ${ }^{18} \mathrm{~F}$ found in this study complies with literature $[12,22,29]$, and can be explained by the larger positron range of ${ }^{68} \mathrm{Ga}$ compared to ${ }^{18} \mathrm{~F}[30]$. Direct compar60 ison of the spatial resolution is difficult, since other groups used different setups to determine the effective spatial resolution [22,23]. Binning is an important factor that influences the spatial resolution, since data of different pixels is combined to enhance the signal and reduce the effects of noise. Although, a binning of 2 reduces the spatial resolution with roughly $10 \%$ ( $1.08 \mathrm{~mm}$ to $1.16 \mathrm{~mm}$, for a 60 s exposure) the gain in signal intensity is fourfold, thus justifying the use of pixel binning in a clinical setting.

To come to a clinical image acquisition protocol that could be used during prostatectomy various acquisition times and pixel binning setting were evaluated. The most optimal setting for clinical ex vivo ${ }^{68} \mathrm{Ga}$ research is considered 120 s and $2 \times 2$ binning, thus acquiring a good spatial resolution within an acceptable timeframe for intraoperative usage and sufficient sensitivity. This is a shorter exposure time and binning factor, as the default setting for ${ }^{18} \mathrm{~F}$ (E300B8) $[16,23]$. Since the light yield is higher with ${ }^{68} \mathrm{Ga}$, the acquisition time and binning factor can be decreased without compromising the quality of the image. For clinical implementation time is important. For rapid assessment, the operation cannot be delayed for more than 10 minutes. Uptake in the prostate tumour, from analysis, shows that the uptake is sufficient for $\mathrm{CLI}$ imaging with ${ }^{68} \mathrm{Ga}-\mathrm{PSMA}-11$, with the required time for prostatectomy and lesion removal. 


\section{IMPLICATIONS FOR CLI DURING PROSTATECTOMY}

${ }^{68} \mathrm{Ga}-\mathrm{PSMA}-11$ tumour uptake measurements were performed in a heterogeneous group of prostate cancer patients. Although large variations are observed in the intensity of PSMA accumulation, we have determined an average and minimal uptake to enable clinically relevant measurements. It was stated that CLI should be able to visualize an average concentration of $3.35 \mathrm{kBq} / \mathrm{mL}$. Based on our in vitro results the detection limit and contrast for ${ }^{68} \mathrm{Ga}$ (with and without tissue) is sufficient to detect this average tumour uptake, even with an exposure time as low as $120 \mathrm{~s}$. The detectable activity concentration with $1 \mathrm{~mm}$ tissue asks for an injection of $2.6 \mathrm{MBq} / \mathrm{kg}{ }^{68} \mathrm{Ga} 45$ minutes prior to $\mathrm{CLI}$, assuming a uniform distribution and water density in the body. Nevertheless, the tumour has 100 times more receptors as benign tissue [19,20], thus injected dose could be lowered for CLI visualisation. Thereby, complying the ${ }^{68} \mathrm{Ga}-\mathrm{PS}-$ MA-11 guidelines for PET imaging [31]. The standard clinical injection of 100 MBq, would be sufficient for intraoperative application with a protocol that fits the clinical requirements. When increasing either the binning or the exposure time, potentially an even lower radioactive dosage can be used. Still, precise patient dosage will be determined in our ongoing clinical feasibility study. Prior studies with ${ }^{18} \mathrm{~F}$ showed that the radiation dose to the surgeon due to the CLI procedure, was $34 \mu \mathrm{Sv}$ per procedure and $2-20 \mu$ Sv per scrub nurse $[14,16]$. The use of ${ }^{68} \mathrm{Ga}-\mathrm{PSMA}-11$ decreases the injected dose, thus improves the radiation safety for both the patient and personnel.

The Cerenkov signal through $\sim 1 \mathrm{~mm}$ tissue surrogate reduced to $73 \%$ of the original signal for ${ }^{18} \mathrm{~F}$, and $62 \%$ for ${ }^{68} \mathrm{Ga}$ according to our measurements. However, this experimental setting does not mimic the exact clinical situation as the chicken breast was not perfused and the optical properties do not comply. The signal is influenced by scattering and absorption in tissue, the attenuation found in the current study (73\%), approximates the value found in literature. Theoretical calculations of $1 \mathrm{~mm}$ tissue, resulted in a decrease in signal intensity of ${ }^{18} \mathrm{~F}$ of $77 \%$ [32]. The same approximation was also made for higher energy nuclides like ${ }^{68} \mathrm{Ga}$. However, the addition of $1 \mathrm{~mm}$ surrogate tissue, resulted in more signal decay in our experiments (62\%) than expected from literature (77\%). Difference in this attenuation percentage could be explained by the influence of the refractive index ( $n$ ). Tissue has a higher $\mathrm{n}$ resulting in a higher number of Cerenkov photons produced, see Equation 1. The corresponding higher mass density results in a higher $\beta$ attenuation cross section and concomitant reduced $\beta$-particle range. Increased density therefore tends to reduce Cerenkov Radiation production efficiency, but for radionuclides that emit relatively 
low energy $\beta$ 's, the increased $n$ dominates resulting in higher light yield for higher density materials. For the high energy $\beta^{\prime} s$ of ${ }^{68} \mathrm{Ga}$, however, the impact of $\mathrm{n}$ is small and the reduction in light yield due to the density effect dominates [22]. Additionally, Glaser et al. showed that a larger refractive index has more impact on the fluence rate of ${ }^{18} \mathrm{~F}$, as ${ }^{68} \mathrm{Ga}[26]$.

Equation 1. The number of Cerenkov photons $N$ emitted per distance travelled $x$, which is derived from the Frank-Tamm equation [21]. $\beta=$ particle velocity, $n=$ refractive index, $\lambda=$ wavelength $(\mathrm{nm}) \mathrm{a}=$ fine structure constant $[a=1 / 137]$.

$$
\frac{\mathrm{d} N}{\mathrm{~d} x}=2 \pi \alpha\left(1-\frac{1}{\beta^{2} \eta^{2}}\right)\left(\frac{1}{\lambda_{1}}-\frac{1}{\lambda_{2}}\right)
$$

\section{LIMITATIONS}

The interference of tissue and blood reduces the obtained signal; however, these influences were not simulated in the current in vitro set up. Therefore, it is difficult to suggest a definite ex vivo acquisition protocol upon solely in vitro measurements. Cerenkov light is predominant in the blue range of the spectrum, and attenuates towards the red part of the spectrum [33]. The weight of the spectrum changes with the influence of tissue, since the blue part is more strongly absorbed [10] as haemoglobin absorbs mostly in this part of the spectrum. For margin assessment accuracy in the penetration depth is important, although not considered in this study. To estimate the penetration depth, it is important to have a phantom with the optical properties of the prostate, since it is influenced by scattering and absorption. The development of a prostate-like-phantom was outside the scope of this paper. For clinical application, the use of numerous filters is recommended, since it enables the possibility to only obtain emitted photons generated near the surface of the tissue. Filters would be needed to determine the depth of the lesion, as the tissue scatters. Scattering is more present in tissue and has a larger influence on light from longer distances [34]. The resulting attenuation is considered positive for margin assessment using $\mathrm{CLI}$, as it benefits superficial imaging to guide complete tumour resection. This manuscript solely focusses on ${ }^{18} \mathrm{~F}$ and ${ }^{68} \mathrm{Ga}$, as ${ }^{68} \mathrm{Ga}-\mathrm{PSMA}-11$ is the most suitable tracer for prostate cancer. However, a variety of isotopes can be used for Cerenkov imaging multiple purposes in medicine $[35,36]$. 


\section{Conclusion}

The performance of the CLI system was determined in vitro using both ${ }^{68} \mathrm{Ga}$ and ${ }^{18} \mathrm{~F}$. The system is linear for both radionuclides at clinically relevant radioactivity concentrations, considering uptake in prostate tumours. ${ }^{68} \mathrm{G}$ a was superior over ${ }^{18} \mathrm{~F}$ in terms of light yield, detectable activity concentration, with and without the addition of chicken tissue. However, as could be expected, the spatial resolution was lower for ${ }^{68} \mathrm{Ga}$. Still, in combination with the short acquisition time (120s) and clinically sufficient detection limit $(1.8 \mathrm{kBq} / \mathrm{mL})$, it seems feasible to obtain ex vivo CLI images for intraoperative margin assessment during prostate cancer surgery using ${ }^{68} \mathrm{Ga}$-PSMA-11. Future studies should confirm the feasibility of this system as intraoperative margin assessor by comparison with histopathology. 


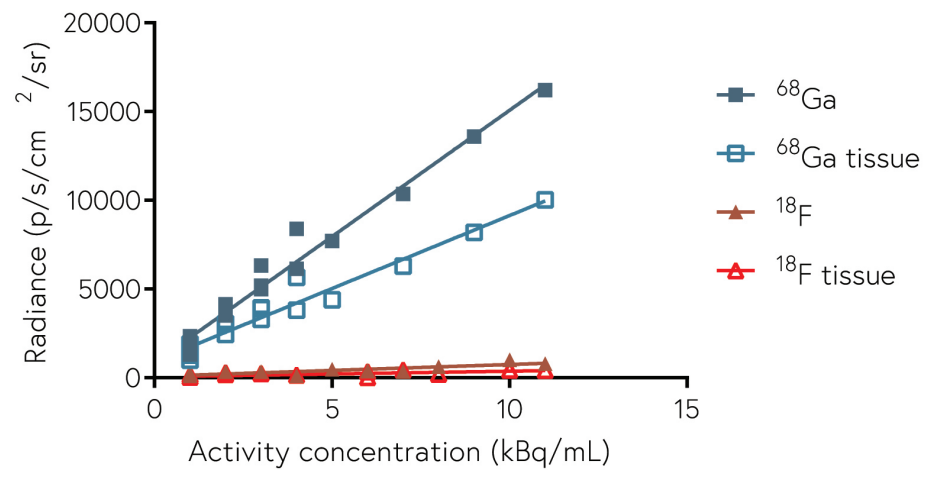

Supplemental Figure 1. Effect of tissue on linearity and signal intensity ${ }^{18} \mathrm{~F}$ and ${ }^{68} \mathrm{Ga}$ within the range of ${ }^{68} \mathrm{Ga}$-PSMA-11 uptake of the prostate tumour according to measurement on the PET/CT scans. Data was acquired with an exposure time of $120 \mathrm{~s}$ and binning $2 \times 2$. The goodness-of-fit $\left[\mathrm{R}^{2}\right]$ is displayed at every fit.

64

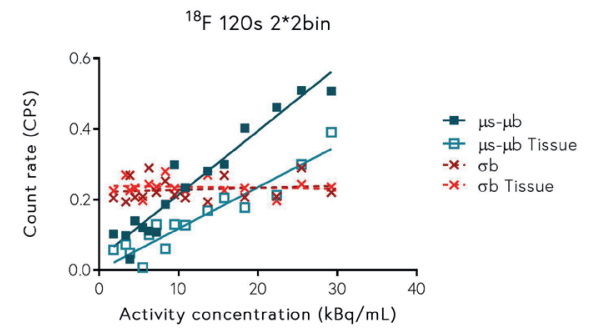

${ }^{68} \mathrm{Ga} 120$ s $2^{\star} 2$ bin

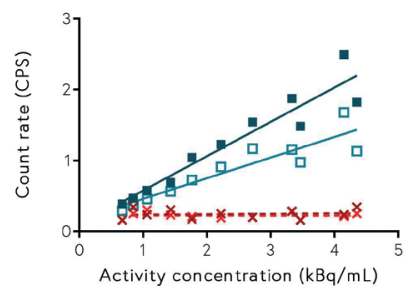

${ }^{18} \mathrm{~F} 300 \mathrm{~s} 8 * 8$ bin

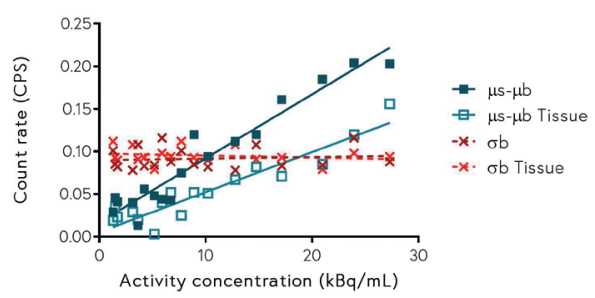

${ }^{68} \mathrm{Ga} 300 \mathrm{~s} 8 * 8$ bin

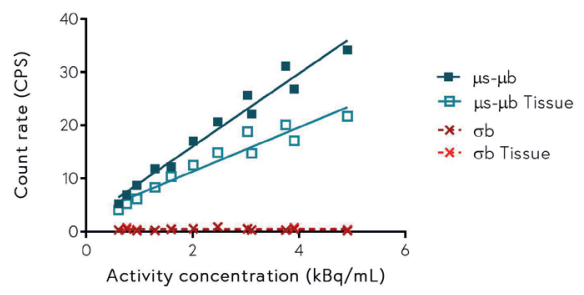

Supplemental Figure 2. Graph representing the signal and the noise floor of ${ }^{18} \mathrm{~F}$ (top] and ${ }^{68} \mathrm{Ga}$ [bottom], with and without overlying tissue. The crossing of the signal with background correction [ $\mu \mathrm{s}-\mu \mathrm{b}]$ and standard deviation of the background signal $(\sigma \mathrm{b})$ represents the minimal detectable activity concentration for $S N R=1$. CPS = counts per second. 

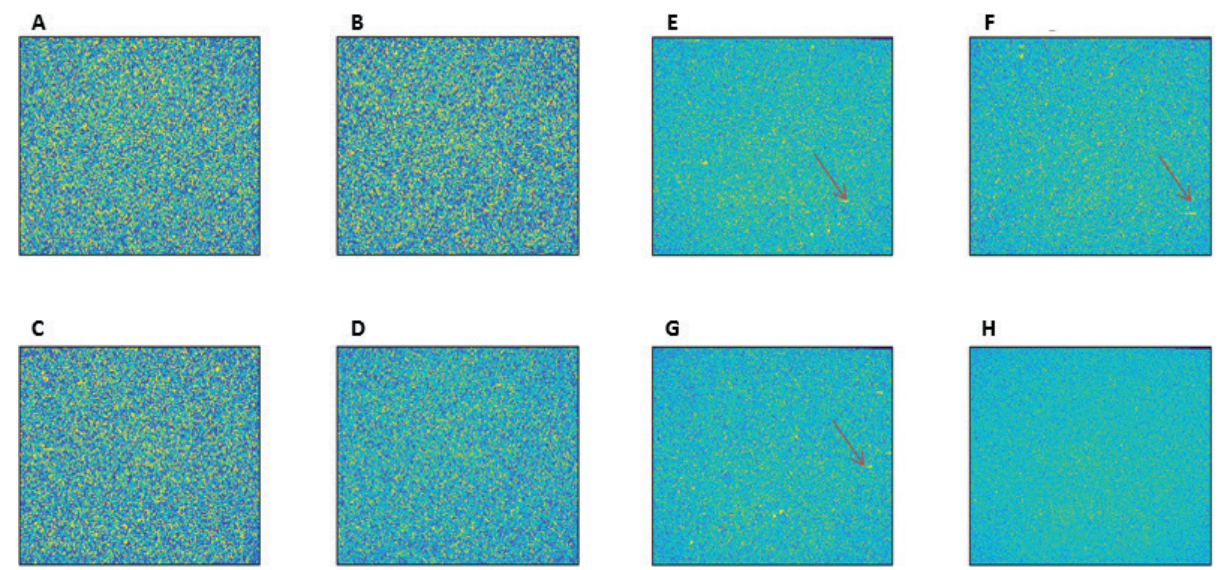

Supplemental Figure 3. Three subsequent images of the uniform flood source $[A-C]$ and the image reconstructed of the median values of the three raw images [D], and the three post-processed images $[E-G]$, with the reconstructed median image $[H]$. The use of the median value $[H]$ reduced the influence of gamma strikes (yellow stripes at the red arrows) in E-F. The same intensity scaling was used in all eight images. 


\section{REFERENCES}

1. Silberstein J, Eastham J. Significance and management of positive surgical margins at the time of radical prostatectomy. Indian J Urol. 2014;30:423- 428

2. Bolla $M$, van Poppel $H$, Tombal B, et al. Postoperative radiotherapy after radical prostatectomy for high-risk prostate cancer: long-term results of a randomised controlled trial (EORTC trial 22911). Lancet. 2012;380:2018-2027.

3. Swanson GP, Riggs MW, Earle JD, et al. Long-term follow-up of radical retropubic prostatectomy for prostate cancer. Eur Urol. 2002;42:212-216.

4. Meeks JJ, Eastham JA. Radical prostatectomy: Positive surgical margins matter. Urol Oncol Semin Orig Investig. 2013;31:974-979.

5. Mauermann J, Fradet V, Lacombe L, et al. The Impact of Solitary and Multiple Positive Surgical Margins on Hard Clinical End Points in 1712 Adjuvant Treatment-Naive pT2-4 NO Radical Prostatectomy Patients. Eur Urol. 2013;64:19-25.

6. Yossepowitch O, Bjartell A, Eastham JA, et al. Positive Surgical Margins in Radical Prostatectomy: Outlining the Problem and Its Long-Term Consequences. Eur Urol. 2009;55:87-99.

7. Novara G, Ficarra V, Mocellin S, et al. Systematic Review and Meta-analysis of Studies Reporting Oncologic Outcome After Robot-assisted Radical Prostatectomy. Eur Urol. 2012;62:382404.

8. Holland JP, Normand G, Ruggiero A, et al. Intraoperative imaging of positron emission tomographic radiotracers using Cerenkov luminescence emissions. Mol Imaging. 2011;10:177-186

9. Robertson R, Germanos MS, Li C, et al. Optical imaging of Cerenkov light generation from positron-emitting radiotracers. Phys Med Biol. 2009;54:N355-365.

10. Spinelli $A E, D^{\prime}$ Ambrosio $D$, Calderan $L$, et al. Cerenkov radiation allows in vivo optical imaging of positron emitting radiotracers. Phys Med Biol. 2010;55:483-495.

11. Grootendorst MR, Cariati M, Kothari A, et al. Cerenkov luminescence imaging (CLI) for image-guided cancer surgery. Clin Transl Imaging. 2016;4:353-366.

12. Mitchell GS, Gill RK, Boucher DL, et al. In vivo Cerenkov luminescence imaging: a new tool for molecular imaging. Philos Trans A Math Phys Eng Sci. 2011;369:4605-4619.

13. Thorek DLJ, Riedl CC, Grimm J. Clinical Cerenkov Luminescence Imaging of ${ }^{18} \mathrm{~F}-\mathrm{FDG}$. J Nucl Med. 2014;55:95-98.

14. Michel C, Freeman A, Jameson C, et al. Intraoperative margin detection using Cerenkov luminescence Imaging during radical prostatectomy-initial results from the PRIME study. Eur J Cancer. 2015;41:PS271.

15. Liu H, Carpenter CM, Jiang H, et al. Intraoperative Imaging of Tumors Using Cerenkov Luminescence Endoscopy: A Feasibility Experimental Study. J Nucl Med. 2012;53:1579-1584.

16. Grootendorst MR, Cariati M, Pinder SE, et al. Intraoperative Assessment of Tumor Resection Margins in Breast-Conserving Surgery Using ${ }^{18} \mathrm{~F}-\mathrm{FDG}$ Cerenkov Luminescence Imaging: A First-in-Human Feasibility Study. J Nucl Med. 2017;58:891-898.

17. Takahashi N, Inoue T, Lee J, et al. The roles of PET and PET/CT in the diagnosis and management of prostate cancer. Oncology. 2008;72:226-233.

18. Effert PJ, Bares R, Handt S, et al. Metabolic imaging of untreated prostate cancer by positron emission tomography with ${ }^{18}$ fluorine-labeled deoxyglucose. J Urol. 1996;155:994-998.

19. Afshar-Oromieh A, Avtzi E, Giesel FL, et al. The diagnostic value of PET/CT imaging with the 
${ }^{68} \mathrm{Ga}$-labelled PSMA ligand HBED-CC in the diagnosis of recurrent prostate cancer. Eur J Nucl Med Mol Imaging. 2014;42:197-209.

20. Afshar-Oromieh A, Zechmann CM, Malcher A, et al. Comparison of PET imaging with a ${ }^{68} \mathrm{Ga}$-labelled PSMA ligand and ${ }^{18} \mathrm{~F}$-choline-based PET/CT for the diagnosis of recurrent prostate cancer. Eur J Nucl Med Mol Imaging. 2014;41:11-20.

21. Gill RK, Mitchell GS, Cherry SR. Computed Cerenkov luminescence yields for radionuclides used in biology and medicine. Phys Med Biol. 2015;60:4263-4280.

22. Beattie BJ, Thorek DLJ, Schmidtlein CR, et al. Quantitative Modeling of Cerenkov Light Production Efficiency from Medical Radionuclides. PLoS One. 2012;7:e31402.

23. Ciarrocchi E, Vanhove C, Descamps B, et al. Performance evaluation of the LightPath imaging system for intraoperative Cerenkov luminescence imaging. Phys Medica. 2018;52:122-128.

24. Hobbie RK, Roth BJ. Medical Use of X Rays In: Intermediate physics for medicine and biology. Springer Science \& Business Media. 2007:451-452

25. Nema NU. NU 1-2012 Performance Measurements of Gamma Cameras. The National Electrical Manufactures Association (NEMA). 2012.

26. Glaser AK, Zhang R, Andreozzi JM, et al. Cherenkov radiation fluence estimates in tissue for molecular imaging and therapy applications. Phys Med Biol. 2015;60:6701-6718

27. Fan D, Zhang $X$, Zhong $L$, et al. ${ }^{68} \mathrm{Ga}$-Labeled 3PRGD2 for Dual PET and Cerenkov Luminescence Imaging of Orthotopic Human Glioblastoma. Bioconjug Chem. 2015;26:1054-1060.

28. Newton MR, Phillips S, Chang SS, et al. Smaller prostate size predicts high grade prostate cancer at final pathology. J Urol. 2010;184:930-937.

29. Ciarrocchi E, Belcari N. Cerenkov luminescence imaging: physics principles and potential applications in biomedical sciences. EJNMMI Phys. 2017;4:14.

30. Champion C, Le Loirec C. Positron follow-up in liquid water: II. Spatial and energetic study for the most important radioisotopes used in PET. Phys Med Biol. 2007;52:6605-6625.

31. Fendler WP, Eiber M, Beheshti $M$, et al. ${ }^{68} \mathrm{Ga}$-PSMA PET/CT: Joint EANM and SNMMI procedure guideline for prostate cancer imaging: version 1.0. Eur J Nucl Med Mol Imaging. 2017:44:1014-1024

32. Chin PTK, Welling MM, Meskers SCJ, et al. Optical imaging as an expansion of nuclear medicine: Cerenkov-based luminescence vs fluorescence-based luminescence. Eur J Nucl Med Mol Imaging. 2013;40:1283-1291.

33. Weissleder R, Ntziachristos V. Shedding light onto live molecular targets. Nat Med. 2003;9:123-128.

34. Axelsson J, Glaser AK, Gladstone DJ, et al. Quantitative Cherenkov emission spectroscopy for tissue oxygenation assessment. Opt Express. 2012;20:5133-5142

35. Lee HJ, Ehlerding EB, Jiang D, et al. Dual-labeled pertuzumab for multimodality image-guided ovarian tumor resection. Am J Cancer Res. 2019;9:1454-1468.

36. Jiménez-Mancilla NP, Isaac-Olivé K, Torres-García E, et al. Theoretical and experimental characterization of emission and transmission spectra of Cerenkov radiation generated by ${ }^{177} \mathrm{Lu}$ in tissue. J Biomed Opt. 2019;24:076002 


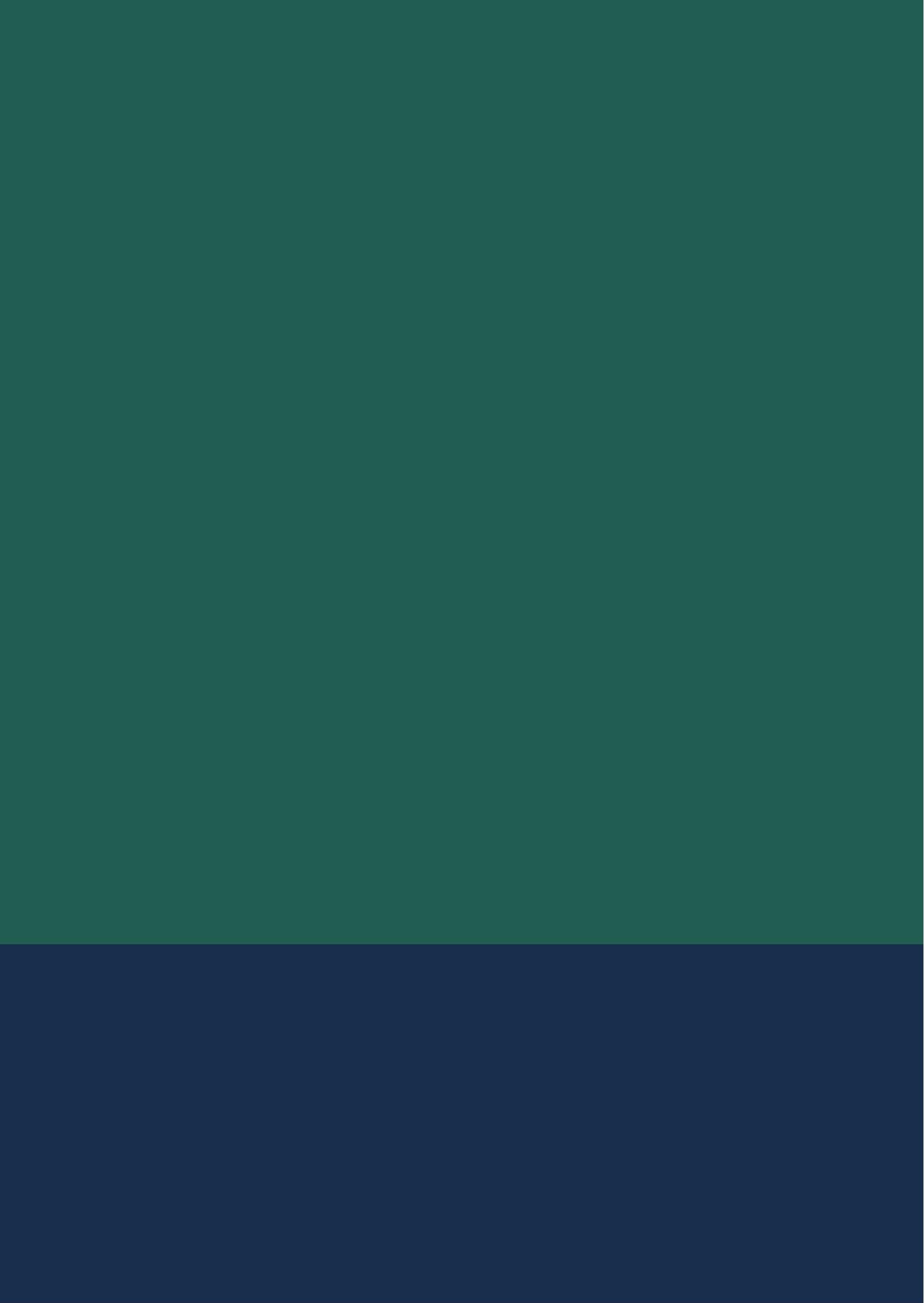




\section{PART II}

\section{USE OF ${ }^{68}$ GA-PSMA-11 PET/CT FOR CLI OPTIMIZATION}




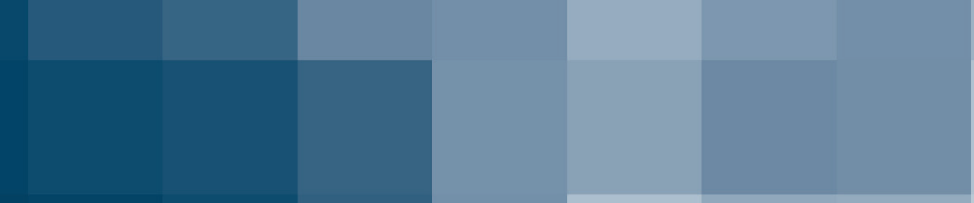




\section{CHAPTER 4}

DAY-TO-DAY VARIABILITY OF ${ }^{68}$ GA-PSMA- 11
ACCUMULATION IN PRIMARY PROSTATE CANCER:

EFFECTS ON TRACER UPTAKE AND VISUAL INTERPRETATION

Judith olde Heuvel

Berlinda J. de Wit-van der Veen Maarten L. Donswijk

Marcel P.M. Stokkel

Cornelis H. Slump

EJNMMI Research 2020;10:132 


\section{Abstract}

\section{INTRODUCTION}

Prostate-specific membrane antigen (PSMA) agents, such as ${ }^{68} \mathrm{Ga}-\mathrm{PSMA}-11$, have an unprecedented accuracy in staging prostate cancer (PCa) and detecting disease recurrence. PSMA PET/CT may also be used for response monitoring by displaying molecular changes, instead of morphological changes alone. However, there is still limited data available on the variability in biodistribution and intra-prostatic uptake of PSMA targeting radiotracers. Therefore, the aim of this study was to assess the repeatability of ${ }^{68} \mathrm{Ga}$-PSMA-11 uptake in primary prostate cancer (PCa) patients in a four-week interval.

\section{Methods}

Twenty-four primary PCa patients were prospectively included, who already were scheduled for ${ }^{68} \mathrm{Ga}-\mathrm{PSMA}-11$ PET/CT scan on clinical indication ( $\geq \mathrm{cT} 3$, Gleason score $\geq 7$ or PSA $\geq 20 \mathrm{ng} / \mathrm{mL}$ ). These patients received two ${ }^{68} \mathrm{Ga}-\mathrm{PSMA}-11 \mathrm{PET} / \mathrm{CT}$ scans with a four week interval. No treatment was started in between the scans. Semi-quantitative measurements $\left(S U L_{\text {max' }}, S U L_{\text {mean' }}\right.$ and $S U L_{\text {peak }}$ ) were determined in the prostate tumour, normal tissues, and blood pool. The repeatability coefficient of every region was determined. All scans were visually analysed by two nuclear medicine physicians.

\section{RESULTS}

Within-subject coefficient of variation of ${ }^{68} \mathrm{Ga}$-PSMA-11 uptake between the two scans was on average $10 \%$ in the prostate tumour, normal tissues (liver, kidney, parotid), and blood pool. The repeatability coefficient of the prostate tumour was $18 \%$ for $S U L_{\text {peak }}$ and $22 \%$ for $S U L_{\text {max }}$. Lesion uptake was visually different in 5 patients, though not clinically relevant.

\section{CONCLUSION}

Results of test-retest ${ }^{68} \mathrm{Ga}$-PSMA-11 PET/CT scans in a four-week interval show that ${ }^{68} \mathrm{Ga}-\mathrm{PSMA}-11$ uptake is repeatable, with a clinical irrelevant variation in tumour and physiological distribution. Based on the presented repeatable uptake, ${ }^{68} \mathrm{Ga}-\mathrm{PSMA}-11$ $\mathrm{PET} / \mathrm{CT}$ scans can potentially be used for disease surveillance and therapy response monitoring. Changes in uptake larger than the RC are therefore likely to reflect actual biological changes in PSMA expression. 


\section{Introduction}

Prostate cancer ( $\mathrm{PC}$ ) is the second most common cancer amongst men in the world, as recorded in 2018 [1]. Molecular imaging of this malignancy either in primary or metastatic setting is presently dominated by the ligands directed to the prostate specific membrane antigen (PSMA). This is a membrane-bound enzyme which is overexpressed in PCa cells compared to benign prostatic tissue by approximately 100- to 1000-fold [2,3]. Gallium-68-labelled PSMA compounds, such as ${ }^{68} \mathrm{Ga}-\mathrm{PS}$ MA-11, is therefore considered a highly tumour specific radiotracer for PCa. Since PSMA agents, have an unprecedented accuracy in recurrent PCa, it has been rapidly adopted in the clinic over the last years $[4,5]$. In staging of primary PCa Hofman et al. recently published a prospective study, showing the higher diagnostic accuracy of ${ }^{68} \mathrm{Ga}$-PSMA-11 PET/CT in men with high-risk primary prostate cancer, as compared to conventional imaging (CT and bone scan) [6]. Which is as well supported with retrospective studies [7-9].

In many solid tumours, active surveillance and response monitoring with fluorine-18 fluorodeoxyglucose $\left({ }^{18} \mathrm{~F}-\mathrm{FDG}\right) \mathrm{PET} / \mathrm{CT}$ is quite common, and adopted in various guidelines [10]. Variations in ${ }^{18} \mathrm{~F}-\mathrm{FDG}$-accumulation can provide valuable information on the activity and efficacy of new cancer therapeutics. The positron emission tomography response criteria in solid tumours (PERCIST) or European organization for research and treatment of cancer (EORTC-) criteria are often used to quantify the response on therapy using ${ }^{18} \mathrm{~F}-\mathrm{FDG}$ PET/CT. These criteria classify the disease status as 'responder', 'progressed', or 'stable' based on changes in the semi-quantitative standard uptake values (SUV), corrected for lean body mass (SUL) [10]. However, ${ }^{18} \mathrm{~F}-\mathrm{FDG} \mathrm{PET} / \mathrm{CT}$ is usually not suitable in PCa, as most tumours show limited FDG-accumulation, especially in hormone-sensitive setting.

Monitoring the response after therapy with ${ }^{68} \mathrm{Ga}$-PSMA-11 PET/CT may be helpful in PCa, yet this approach is not validated yet [11]. As with FDG-accumulation, the extent and intensity of the PSMA-uptake can be compared between scans to quantify response, and when deemed necessary adjust therapy. However, not many papers are published about response monitoring or active surveillance using ${ }^{68} \mathrm{Ga}$-PSMA-11 PET/CT [12]. One study by Gupta et al. compared the functional criteria PERCIST V1.0 and EORTC in ${ }^{68} \mathrm{Ga}-\mathrm{PSMA}-11$ PET/CT with the morphological criteria according to RECIST V1.1 in patients with metastatic PCa and biochemical progression [13]. 
According to this study, molecular imaging criteria performed best in detecting progression based on changes of $25 \%$ SUV $_{\text {mean }}$ (EORTC) or $30 \%$ SUL $_{\text {peak }}$ (PERCIST) after hormone treatment [13]. However, the study does not describe if the biological variation in ${ }^{68} \mathrm{Ga}-\mathrm{PSMA}-11$ uptake is comparable to that of ${ }^{18} \mathrm{~F}-\mathrm{FDG}$, and if the same cut-off values apply. While only changes that exceed the normal variability should be interpreted as treatment response or disease progression. Although two additional studies assessed this test-retest repeatability in metastatic prostate cancer using ${ }^{68} \mathrm{Ga}$-PSMA-11 [14] and ${ }^{18} \mathrm{~F}-\mathrm{DCFP} y \mathrm{~L}$ [15], no studies in the primary setting are performed to this day, as far as we are aware of. Therefore, the aim of this study was to assess the day-to-day variability of ${ }^{68} \mathrm{Ga}$-PSMA-11 uptake and visual interpretation in patients with primary prostate cancer. 


\section{Methods}

\section{PATIENTS}

This prospective clinical study was performed at the Netherlands Cancer Institute (Amsterdam, the Netherlands). The study protocol was approved by the local medical ethics committee (NL8263 at trialregister.nl) and all patients provided written informed consent. Men (18 year) with biopsy-proven PCa and a clinical indication to perform a ${ }^{68} \mathrm{Ga}$-PSMA-11 PET/CT scan (e.g. either $\geq c T 3$, Gleason score $\geq 7$ or PSA $\geq 20$ $\mathrm{ng} / \mathrm{mL}$ ) were eligible. Patients were excluded if no elevated ${ }^{68} \mathrm{Ga}$-PSMA-11 uptake was visible in the primary prostate tumour on the first scan, or when treatment was started in between the two scans.

\section{STUDY PROTOCOL}

The first ${ }^{68} \mathrm{Ga}-\mathrm{PSMA}-11 \mathrm{PET} / \mathrm{CT}$ scan was performed on clinical indication based on the aforementioned criteria. Both PET/CT scans were performed according to the same local clinical protocol, consisting of adequate oral hydration before an intravenous bolus injection of $100 \pm 10 \mathrm{MBq}{ }^{68} \mathrm{Ga}-\mathrm{Glu}$-urea-Lys-(Ahx)-HBED-CC $\left({ }^{68} \mathrm{Ga}\right.$-PSMA-11), which was radiolabelled in-house using a fully automated system (Scintomics $\mathrm{GmbH}$, Fürstenfeldbruck, Germany). After an incubation time of $45 \pm 5$ minutes, acquisitions were performed on a Vereos digital PET/CT system (Philips, Best, the Netherlands). Acquisition time was 3 minutes per bed position ( $\mathrm{min} / \mathrm{bp}$ ) for the pelvic area and $2 \mathrm{~min} / \mathrm{bp}$ towards base of skull. In the last phase of the study, clinical protocol changed to an administered activity of $150 \pm 15 \mathrm{MBq}{ }^{68} \mathrm{Ga}-\mathrm{PSMA}-11$ with acquisitions of $4.5 \mathrm{~min} / \mathrm{bp}$ around the pelvis to improve image quality. CTs were acquired for attenuation correction and anatomical correlation. In general, no furosemide was given to the patients. The second scan was scheduled roughly 4 weeks later, for which deviations in administered activity within $\pm 10 \%$ and in time between injection and acquisition of within \pm 5 min are aimed for.

\section{IMAGE RECONSTRUCTION AND ANALYSIS}

Data was reconstructed at 3 iterations, 8 subsets with Gaussian blurring $(3 \mathrm{~mm})$ and voxel size of $2 \times 2 \times 2 \mathrm{~mm}$ (matrix size $512 \times 512$ ). Semi-quantitative measures were determined using either Osirix MD (Pixmeo SARL, Switzerland) or 3D Slicer (www. slicer.org). Spherical volumes-of-interest (VOI) were drawn to determine average uptake in the primary tumour $(\varnothing 1.7 \mathrm{~cm})$, normal tissues (i.e., right parotid gland $\varnothing 2.5$ $\mathrm{cm}$, liver $\varnothing 5 \mathrm{~cm}$, right kidney $\varnothing 3.5 \mathrm{~cm}$, $4^{\text {th }}$ lumbar vertebra bone marrow $\varnothing 2.5 \mathrm{~cm}$ ) 
and blood pool activity in the abdominal aorta and ascending aorta $(\varnothing 1.7 \mathrm{~cm})$. Minimal VOI diameter of $1.7 \mathrm{~cm}$ was chosen to reduce partial volume effect. If present, ${ }^{68} \mathrm{Ga}-\mathrm{PSMA}-11$ positive metastatic bone lesions or lymph nodes were included in the analysis. The blood pool was used as reference value $[12,16]$. The standard uptake value corrected for lean body mass (SUL) was used for quantitative analyses [17,18].

In the primary tumour, $S U L_{\text {peak }}$ was defined as the $1 \mathrm{~cm}^{3}$ with the highest activity concentration in the VOI. If multifocal lesions in the prostate were present, the $\mathrm{SUL}_{\text {peak }}$ of the hottest lesion was shown. The tumour-to-blood ratios (TBR) were also determined, as they were found to best describe the tumour tracer uptake [19]. The relative difference between scan 1 and 2 was calculated for all indices.

\section{VISUAL ASSESSMENT}

Visual analysis was performed by two nuclear medicine physicians (MLD and MPMS) with experience in reading ${ }^{68} \mathrm{Ga}-\mathrm{PSMA}-11 \mathrm{PET} / \mathrm{CT}$ scans to assess any visual differences between the acquisitions. Both physicians were blinded to clinical parameters, possible other imaging and to which scan was made first and second. If visual differences were noted, the location of these differences was recorded, and scored 76 as deviation in either bio-distribution or lesion uptake. Any disagreement between the physicians was settled in consensus.

\section{StATISTICAL ANALYSIS}

All statistical analyses were performed using SPSS 25 (IBM, Armonk, USA). The difference between tracer uptake time and injected activities of the two scans was assessed using paired T-tests. Repeatability was evaluated using different metrics, difference (d), relative difference $(D)$, repeatability coefficient $(R C)$, and within-subject coefficient of variation ( $w C V$ ) $[20,21]$. The $w C V$ is the variance of the repeated measurements of individual subjects. The RC denotes the absolute difference between repeated measurements, which lies within the $95 \%$ confidence interval. The smaller these value the better the repeatability. 


\section{Results}

\section{PAtients And PET IMAgIng}

A total of twenty-four patients were included in this study. Two patients were excluded, as no elevated PSMA expression was observed in the prostate on the first scan. Patient demographics are shown in Table 1. The average injected activity was not statistically different between the two scans (99.8 MBq [range 81-113] and 103.8 $\mathrm{MBq}$ [range 96-110]; $p=0.06$, for $n=20)$, as was the interval between radiotracer injection and scan (45.1 min [range 38-57] and $46.1 \mathrm{~min}$ [range 41-64]; $p=0.42$, for $\mathrm{n}=22)$. The average time difference between administration and acquisition for the two scans was $4 \mathrm{~min}$ (0-19 $\mathrm{min}$ ) and 7\% (0-22\%) difference in injected activity. Four patients violated the $10 \%$ difference in injected dose (22\% \& $28 \%$ ) or 5 minute deviation ( 9 \& $19 \mathrm{~min}$ ) in tracer incubation time between the two scans. In two patients, the new protocol was applied, with an injected activity at scan 1 of 136.7 and 146.9 $\mathrm{MBq}$ and at scan 2130.8 and $139.6 \mathrm{MBq}$, respectively.

Table 1. Demographics of the patients included in this study. Data is shown as absolute value [percentage) or as mean [range]. iPSA- initial Prostate Specific Antigen

\begin{tabular}{|c|c|c|}
\hline & & Number $(\%)$ or mean (range) \\
\hline Age (years) & & $71(53-81)$ \\
\hline \multirow[t]{5}{*}{ Gleason score } & 6 & $1(5 \%)$ \\
\hline & $3+4=7$ & $2(9 \%)$ \\
\hline & $4+3=7$ & $9(41 \%)$ \\
\hline & 8 & $5(23 \%)$ \\
\hline & $9+10$ & $5(23 \%)$ \\
\hline \multirow[t]{3}{*}{ iPSA (ng/mL) } & $<10$ & $11(50 \%)$ \\
\hline & $10-20$ & $8(36 \%)$ \\
\hline & $>20$ & $2(9 \%)$ \\
\hline \multirow[t]{3}{*}{$\mathrm{T}$} & cT1 & $9(41 \%)$ \\
\hline & cT2 & $7(32 \%)$ \\
\hline & cT3 & $6(27 \%)$ \\
\hline \multirow[t]{2}{*}{$N$} & NO & $21(95 \%)$ \\
\hline & N1 & $1(5 \%)$ \\
\hline \multirow[t]{2}{*}{ M } & MO & $21(95 \%)$ \\
\hline & M1 & $1(5 \%)$ \\
\hline Prostate volume (cc) on MRI & & $48.0(22-88)$ \\
\hline
\end{tabular}




\section{${ }^{68} \mathrm{Ga}-\mathrm{PSMA}-11$ uptake in normal tissue and blood pool}

The average $S U L_{\text {max }}$ and $S U L_{\text {mean' }}$ the (relative) differences, $w C V$ and RC of every organ are displayed in Table 2. In the blood pool, a relative mean difference in SUL of $1 \%$ (range -29.2 to $+24.5 \%$ ) in ${ }^{68} \mathrm{Ga}$-PSMA-11 uptake was observed. The SUL difference between the bladder (RC: $122 \%$ ) and kidney (RC: $24 \%$ ) are larger than in the rest of the organs. With a $\mathrm{RC}$ of $18 \%$, the smallest difference was observed in the liver. The effects on $\mathrm{RC}$ due to protocol violation ( $\mathrm{n}=4$ patients) and change in acquisition protocol ( $n=2$ patients) are displayed in Supplemental Figure $1 \& 2$. In one patient the parotid gland was not included in the analysis, as quantification was not accurate due to head movement. 
Table 2. SUL $\mathrm{max}, \mathrm{SUL}_{\text {mean }}, \mathrm{SUL} \mathrm{L}_{\text {peak }}$ for normal tissue and the prostate tumour (mean \pm range). Next the absolute difference, the relative difference between scan 1 and scan 2 . The within subjects coefficient of variation (wCV) in \%, the coefficient of repeatability [RC], both in \% as in absolute SUL values.

\begin{tabular}{|c|c|c|c|c|c|c|c|}
\hline Organ & Metric & $\begin{array}{c}\text { Average } \\
\text { SUL }\end{array}$ & $\begin{array}{l}\text { Difference in } \\
\text { SUL scan } 2-1\end{array}$ & $\begin{array}{c}\text { Relative } \\
\text { difference (\%) }\end{array}$ & $\begin{array}{l}\text { wCV } \\
(\%)\end{array}$ & $\begin{array}{c}\mathrm{RC} \\
\text { (SUL) }\end{array}$ & $\begin{array}{l}\mathrm{RC} \\
\text { (\%) }\end{array}$ \\
\hline \multirow[t]{4}{*}{ Parotid Gland } & $S U L_{\max }$ & $15.1 \pm 3.3$ & $0.5 \pm 1.2$ & $4.1 \pm 9.0$ & 11.3 & 2.3 & 17.0 \\
\hline & & $(10.1,23.1)$ & $(-1.4,2.6)$ & $(-9,21)$ & & & \\
\hline & $S U L_{\text {mean }}$ & $9.6 \pm 1.9$ & $0.2 \pm 1.2$ & $3.9 \pm 11.9$ & 8.4 & 2.3 & 23.4 \\
\hline & & $(5.8,13.7)$ & $(-1.9,2.3)$ & $(-15,26)$ & & & \\
\hline \multirow[t]{4}{*}{ Aortic arch } & $\mathrm{SUL}_{\max }$ & $1.8 \pm 0.3$ & $0.0 \pm 0.3$ & $-0.2 \pm 18.6$ & 13.2 & 0.6 & 36.5 \\
\hline & & $(1.0,2.5)$ & $(-0.6,0.8)$ & $(-31,38)$ & & & \\
\hline & $S U L_{\text {mean }}$ & $0.9 \pm 0.1$ & $0.0 \pm 0.1$ & $0.2 \pm 12.2$ & 9.6 & 0.2 & 26.6 \\
\hline & & $(0.5,1.2)$ & $(-0.3,0.1)$ & $(-29,25)$ & & & \\
\hline \multirow[t]{4}{*}{ Liver } & $S U L_{\max }$ & $7.2 \pm 1.5$ & $-0.5 \pm 1.3$ & $-7.1 \pm 15.8$ & 6.5 & 1.4 & 31.1 \\
\hline & & $(5.0,9.6)$ & $(-5.5,1.3)$ & $(-63,21)$ & & & \\
\hline & $S U L_{\text {mean }}$ & $3.7 \pm 0.8$ & $0.3 \pm 0.4$ & $-3.4 \pm 9.2$ & 11.2 & 0.8 & 17.9 \\
\hline & & $(2.3,5.5)$ & $(-1.1,0.6)$ & $(-25,13)$ & & & \\
\hline \multirow[t]{4}{*}{ Kidney } & $S U L_{\max }$ & $42.5 \pm 6.9$ & $0.3 \pm 4.5$ & $1.0 \pm 11.3$ & 8.6 & 8.5 & 22.2 \\
\hline & & $(27.1,56.7)$ & $(-6.4,9.4)$ & $(-17,29)$ & & & \\
\hline & $S U L_{\text {mean }}$ & $19.5 \pm 3.8$ & $-0.5 \pm 2.4$ & $-2.4 \pm 15.0$ & 8.0 & 4.7 & 23.9 \\
\hline & & $(11.6,28.1)$ & $(-5.2,3.4)$ & $(-25,13)$ & & & \\
\hline \multirow[t]{4}{*}{ A. Abdominalis } & $S U L_{\max }$ & $1.9 \pm 0.4$ & $0.0 \pm 0.4$ & $2.6 \pm 18.2$ & 12.9 & 0.7 & 35.6 \\
\hline & & $(1.2,2.8)$ & $(-0.6,0.8)$ & $(-28,44)$ & & & \\
\hline & $S U L_{\text {mean }}$ & $0.9 \pm 0.2$ & $0.0 \pm 0.1$ & $1.8 \pm 14.3$ & 10.7 & 0.3 & 29.7 \\
\hline & & $(0.4,1.1)$ & $(-0.2,0.3)$ & $(-29,35)$ & & & \\
\hline \multirow[t]{4}{*}{ Bone } & $\mathrm{SUL}_{\max }$ & $1.5 \pm 0.4$ & $0.1 \pm 0.4$ & $3.1 \pm 25.2$ & 17.8 & 0.8 & 49.4 \\
\hline & & $(0.9,2.5)$ & $(-0.7,0.8)$ & $(-48,45)$ & & & \\
\hline & $S U L_{\text {mean }}$ & $0.5 \pm 0.2$ & $0.0 \pm 0.1$ & $-6.0 \pm 14.0$ & 10.0 & 0.2 & 27.7 \\
\hline & & $(0.3,0.9)$ & $(-0.2,0.1)$ & $(-33,17)$ & & & \\
\hline \multirow[t]{4}{*}{ Bladder } & $S U L_{\max }$ & $12.5 \pm 8.9$ & $-1.6 \pm 8.9$ & $2.0 \pm 58.1$ & 41.1 & 17.2 & 113.8 \\
\hline & & $(3.3,43.7)$ & $(-40.2,12.4)$ & $(-112,113)$ & & & \\
\hline & $S U L_{\text {mean }}$ & $7.7 \pm 5.2$ & $-0.3 \pm 6.4$ & & 43.9 & 12.3 & 121.8 \\
\hline & & $(1.9,32.2)$ & $(-7.7,22.6)$ & $(-108,128)$ & & & \\
\hline \multirow{4}{*}{$\begin{array}{l}\text { Prostate } \\
\text { tumour }\end{array}$} & $S U L_{\max }$ & $10.7 \pm 7.3$ & $-0.2 \pm 1.1$ & $-0.5 \pm 11.2$ & 7.9 & 2.2 & 21.9 \\
\hline & & $(3.1,32.3)$ & $(-2.2,2.5)$ & $(-18,21)$ & & & \\
\hline & $S U L_{\text {peak }}$ & $6.2 \pm 3.5$ & $0.0 \pm 0.6$ & $1.2 \pm 9.2$ & 6.5 & 1.1 & 18.1 \\
\hline & & $(2.6,13.3)$ & $(-1.2,1.4)$ & $(-15,19)$ & & & \\
\hline \multirow[t]{4}{*}{ TBR } & $S U L_{\max }$ & $5.9 \pm 4.2$ & $-0.2 \pm 1.2$ & $-1.0 \pm 19.7$ & 13.8 & 2.4 & 38.4 \\
\hline & & $(1.7,17.8)$ & $(-3.3,1.9)$ & $(-43,42)$ & & & \\
\hline & $S U L_{\text {peak }}$ & $5.4 \pm 1.3$ & $0.2 \pm 0.6$ & $5.7 \pm 17.1$ & 9.9 & 1.6 & 27.5 \\
\hline & & $(2.1,12.5)$ & $(-1.0,2.4)$ & $(-19.5,27.6)$ & & & \\
\hline
\end{tabular}

Values are displayed as mean \pm standard deviation (min, max). Average SUL of each site was calculated as mean over all subjects and all scans. Difference SUL and the relative difference is calculated as the group average of the difference. 


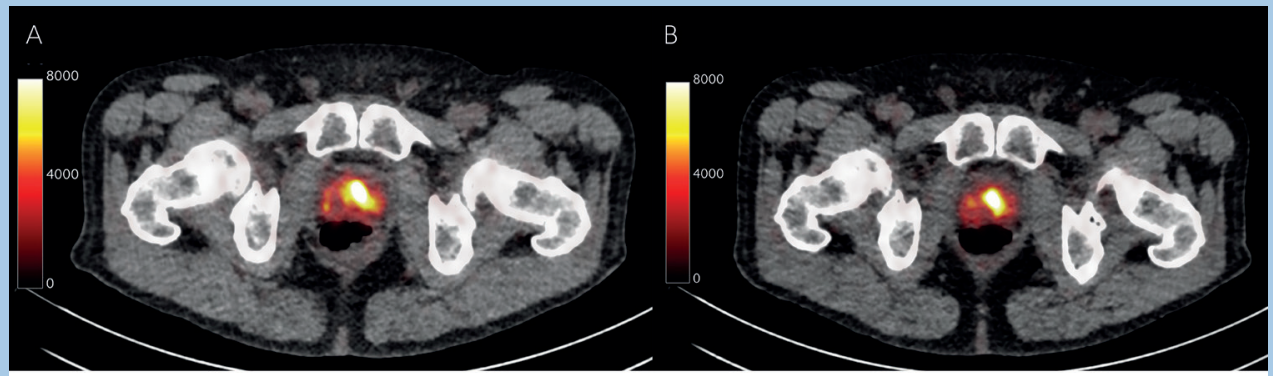

C

D

$E$
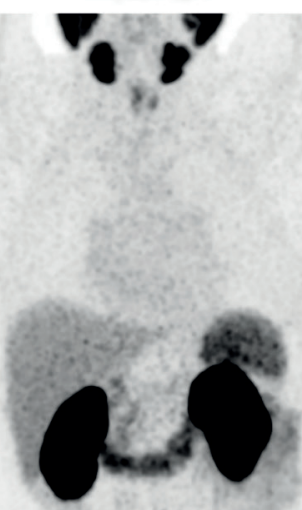

11
$\mathrm{F}$

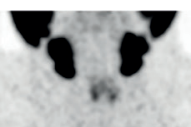

$+$

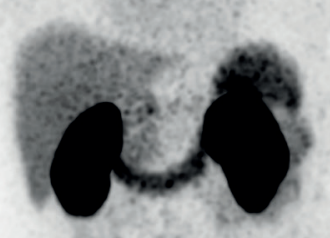

Qutsers

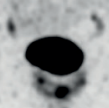

Figure 1. Example of a large difference between $S_{U} L_{\text {peak }}$ between scan $1[A, C, E]$ and $\operatorname{scan} 2[B, D, F]$, the difference in $\mathrm{SUL}_{\text {peak }}$ between both scans is 1.4 


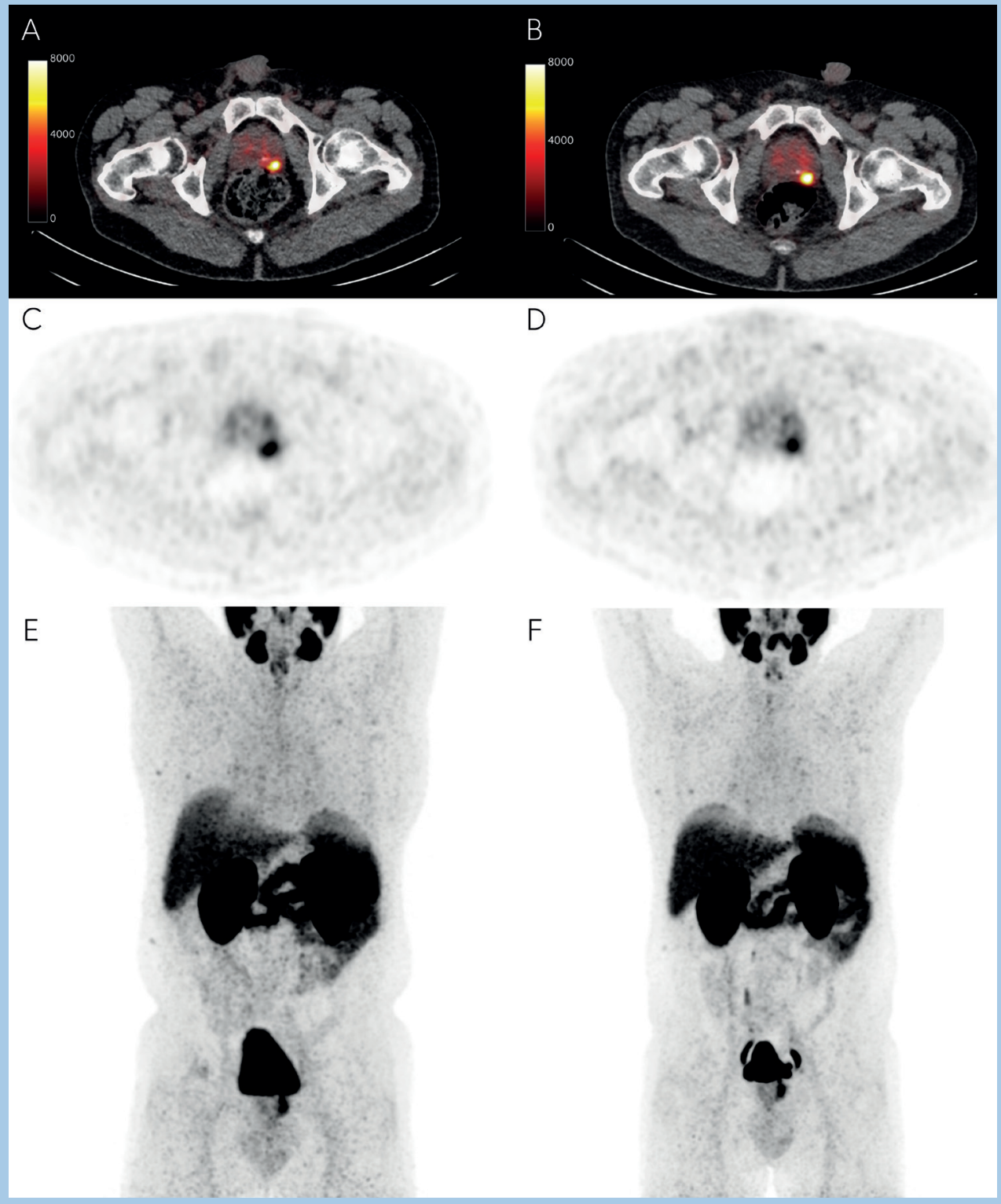

Figure 2. Example of a small difference between $S U L_{\text {peak }}$ between $\operatorname{scan} 1[A, C, E]$ and $\operatorname{scan} 2[B, D, F]$, the difference in $S U L_{\text {peak }}$ between both scans is 0.4 


\section{${ }^{68}$ GA-PSMA-11 UPTAKE IN PRIMARY TUMOUR}

On average, the relative mean difference in $\mathrm{SUL}_{\text {peak }}$ of the prostate tumour between the two scans was $1.2 \%$ (range -14.5 to $+18.6 \%$ ). The RC of SUL max, $_{\text {, }}$ and SUL $L_{\text {peak }}$ was 2.1 $(21.9 \%)$ and $1.1(18.1 \%)$, respectively. In general the $S U L_{\max }$ is somewhat higher due to the larger impact of image noise, compared to $S U L_{\text {peak }}$. Figure 3 shows the absolute differences between the two scans for both metrics against the average value of the two (i.e., Bland-Altman plot). Figure 1 and 2 show two examples of ${ }^{68} \mathrm{Ga}-\mathrm{PSMA}-11$ PET/CTs with relative small and large variation in the $S U L_{\text {peak }}$ of the primary tumour between the two scans. Note that though there are quantitative differences, the distribution pattern within the prostate is comparable in both examples. TBRs had a repeatability coefficient of $38.4 \%$ of $S U L_{\text {max }^{\prime}}$ with an average difference of $-1 \%$ (range -43 to $+41.5 \%$ ). The effects on RC due to protocol violation ( $n=4$ patients) and change in acquisition protocol ( $n=2$ patients) are displayed in Supplemental Figure $1 \& 2$.

Figure 3 shows the Bland Altman plots of the primary tumour, normal tissue and the blood pool. These plots show no clear association, in other words, that the repeatability is equally robust across a wide SUL range in normal tissue and in the prostate. 

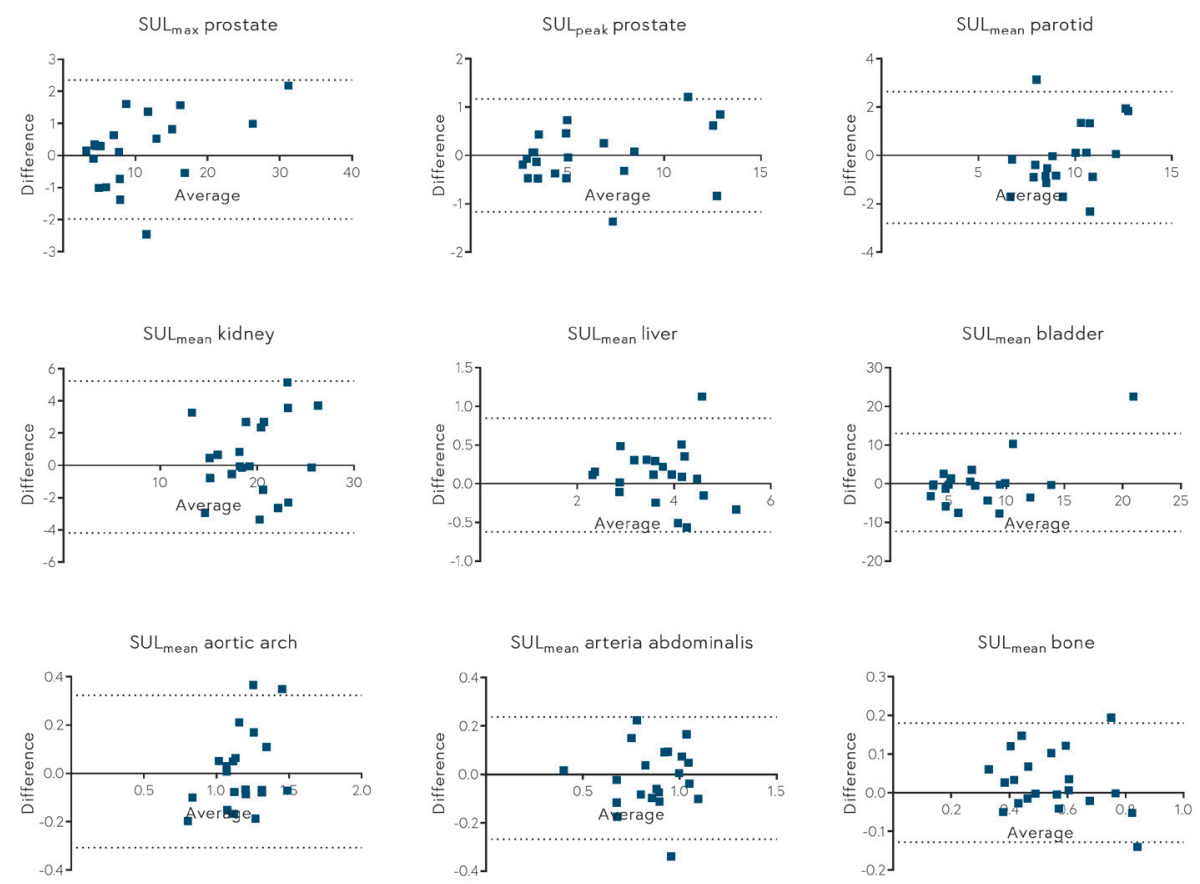

Figure 3. Bland-Altman plots of the absolute difference in $S U L_{\text {max' peak }}$ of the prostate tumour, and $S U L_{\text {mean }}$ of the parotid, liver, bladder, kidney, blood pool (abdominal aorta and ascending aorta), and bone. Horizontal lines represent the mean differences and the 95\% confidence intervals of limits

\section{VISUAL ASSESSMENT}

Visually there was no difference between the two scans in 17 of the 22 cases with respect to the detectability and extent of the lesions. In three patients, visual differences between the two scans were noted with regard to the primary prostate lesion. In two other In these particular cased, the visual differences did not have any impact on the clinical staging and subsequent treatment plan. With regard to the biodistribution, no visual differences in radiotracer accumulation were observed except for differences in bladder and urinary tract activity in six patients. 


\section{Discussion}

To our knowledge, repeatability of ${ }^{68} \mathrm{Ga}-\mathrm{PSMA}-11 \mathrm{PET} / \mathrm{CT}$ scans in the primary PCa setting has not been investigated before. This information is essential to perform response monitoring based on PSMA PET/CT. Therefore, the aim of this study was to assess the repeatability of ${ }^{68} \mathrm{Ga}$-PSMA-11 PET/CT scan in primary PCa patients in a four-week interval.

The repeatability coefficient of SUL $L_{\text {peak }}$ in the prostate tumour was $18.1 \%$, suggesting that below this value, the absolute difference between two scans in one patient (under the same circumstances) should fall within $95 \%$ probability. If the relative difference in $S U L_{\text {peak }}$ is larger than $\pm 18 \%$, then the difference is more likely to be explained by true changes then by measurement errors. The absolute RC in the prostate tumour is probably less useful than the percentage, as there is a broad range in uptake between patients. The SUL $L_{\text {mean }}$ of normal tissue has a RC of $23.8 \%$ on average. In general, the RC of the SUL $L_{\text {max }}$ is higher than the RC of SUL $L_{\text {mean }}$ since SULmax is more susceptible to noise. The RC of the SUL $L_{\text {mean }}$ of the blood pool was $26 \%$. However, due to the very low radiotracer activity in the bone and blood pool, small 84 absolute differences in $S U L_{\text {mean }}$ can already lead to a large percentage difference. Thus, in these organs it is more informative to look at the repeatability coefficient of the absolute difference, which is 0.2 for the blood pool and 0.2 for bone.

The visual differences between the two scans were not considered clinically relevant by the expert readers, and were predominantly related to a variable urinary excretion or variances in noise levels. Particularly, in three patients the activity in the prostate was noisy, therefore the primary tumour was difficult to distinguish from the background, probably explaining the initial inter-observer differences in visual assessment. Next, in one of the patients, the visual appearance of a lymph node was different, which might have been caused by a larger (22\%) deviation in the injected dose between the two scan points in that particular case. Still, in this patient the tumour and other organs were not visually different.

The current study had quite a similar set up as previous studies described in literature, but there are some relevant differences [14,15]. Previous studies looked at metastatic patients or ${ }^{18} \mathrm{~F}-\mathrm{DCFP} y \mathrm{~L}$ instead of ${ }^{68} \mathrm{Ga}$-PSMA-11. Given the similar bio distribution, findings for ${ }^{68} \mathrm{Ga}-\mathrm{PSMA}-11$ may probably be generalized to ${ }^{18} \mathrm{~F}-\mathrm{DCFPyL}$ [22]. Noteworthy is that ${ }^{18} \mathrm{~F}-\mathrm{PSMA}-1007$ has a distinctly different bio distribution 
than the latter radiotracers [23]. Also, most other studies quantified uptake with body-weight corrected SUV instead of SUL, yet the RC of SUL $L_{\max }$ and SUV ${ }_{\max }$ within the same patient in a test-retest setting is comparable. The RC of SUL $L_{\max }$ of the prostate lesion in our study was $21.9 \%$. Pollard et al. reported $30.3 \%$ and Jansen et al. $31.0 \%$ SUV $_{\max }[14,15]$. The WCV of the prostate tumour in our study is $7.9 \%$ for SUL$\max ^{\prime}$ compared to $10.9 \%$ for SUV ${ }_{\max }$ reported in the study by Pollard et al. [14]. These studies investigated patients with metastatic lesions as opposed to the patients with primary prostate cancer in our study. Metastatic lesions have a larger variation in PSMA receptor expression and general expansion of disease between patients than primary PCa tumours, thus possibly resulting in a higher RC than ours [24]. When comparing our TBR findings to Jansen et al. [15], the RC is within the same range (38.4\% vs 37.3\%). The RC of the SUL is smaller than the RC of the TBR $(21.9 \%$ vs 38.4\% SUL max $_{\text {ms }}$ TBR $_{\text {max }}$ ), probably because TBR adds the SUL variation both the blood pool and the tumour. This was found by Jansen et al. as well [15]. In prior studies a shorter window of not more than 7 days for repeatability was used. Due to the fact that prostate cancer is generally an indolent cancer, a four-week interval was considered reliable, as shown in present study.

In general, the SUL $L_{\max }$ values of normal tissues found in literature for PSMA PET/CT scans are comparable to ours [16,25-27]. The differences between the two scans are largest in the ureter and bladder, which can be explained by variations in urine volume and radiotracer activity in the bladder. Though patients were not allowed to receive furosemide according to study protocol study, two patients had a protocol violation and received furosemide before one acquisition, resulting in a clear difference in bladder radiotracer activity (i.e., 2.1 vs $9.6 \mathrm{SUL}_{\text {mean }}$ ). The differences between two scans is lowest in the liver, concordant to the findings of Li et al. [28]. The uptake variation of the parotid is on average repeatable (23.4\%), although there is a large range, and comparable to the results of Pollard et al. (26.5\%) [14]. However, comparing the SUL ${ }_{\text {mean }}$ to ${ }^{18} \mathrm{~F}-\mathrm{DCFPyL}$ found by Li et al., the parotid and kidney SUL${ }_{\text {mean }}$ were lower than our findings, still the SUL $L_{\text {mean }}$ of the liver was equal [28].

The repeatability of ${ }^{68} \mathrm{Ga}$-PSMA-11 uptake in prostatic lesions is not entirely comparable to previous findings of ${ }^{18} \mathrm{~F}-\mathrm{FDG}$ uptake in malignancies [21]. However, ${ }^{18} \mathrm{~F}-\mathrm{FDG}$ scans do require a more concise preparation and patients' adherence to the protocol [29]. In the day-to-day clinical setting, patients are likely to have variation in for instance blood glucose levels or physical activity, thus directly resulting in a less 
reproducible ${ }^{18} \mathrm{~F}-\mathrm{FDG}$ uptake. The advantage of ${ }^{68} \mathrm{Ga}-\mathrm{PSMA}-11 \mathrm{PET} / \mathrm{CT}$ is that signal intensity depends primarily on the number of PSMA-expressing cells and expression density per cell [30]. In contrast to ${ }^{18} \mathrm{~F}-\mathrm{FDG}$, which accumulates to some extent in most tissues in the body, PSMA-ligands tent to accumulate only in specific tissues. Sahakyan et al. found that variability in the liver and kidneys can be caused by intrapatient factors (i.e., time of day, recent meals, hydration status), and that interpatient factors (i.e., weight, height, body composition, medical comorbidities) can influence uptake in the salivary glands [31]. Nonetheless, these influences were described in patients who underwent therapy, so it is difficult to distinguish between therapeutic effects and day-to-day physiological variations.

All these characteristics aid in repeatable quantitative ${ }^{68} \mathrm{Ga}-\mathrm{PSMA}-11$ uptake in PCa lesions, and so it should allow for stable follow-up monitoring of the disease. A change in PSMA uptake in the tumour more than $18 \%$ as mentioned before, may indicate either disease progression or treatment response. In a preclinical setting, PSMA expression is already used for response monitoring in Taxane-based chemotherapy [32]. Note that careful image interpretation is needed when describing an in- or decrease of PSMA uptake [33]. Androgen deprivation therapy can influence the PSMA expression, where up or downregulation is not unambiguously affected by type and duration of medication [34-36]. If PSMA PET/CT is used for response monitoring of radionuclide therapy, the tumour sink effect cannot be neglected [37].

\section{LIMITATIONS}

Our study has some limitations that need some further elaborations. First, there was an alteration in the clinical imaging protocol while performing this study. The prescribed activity of ${ }^{68} \mathrm{Ga}$-PSMA-11 was increased from $100 \mathrm{MBq}(n=20)$ to 150 $\mathrm{MBq}(\mathrm{n}=2)$ in order to improve the image quality of PET/CT images in our institute. Though the effects on the SUL $L_{\text {mean }}$ and $S U L_{\text {peak }} R C$-values are limited (Supplemental Figure 1), the effect is somewhat larger in $\mathrm{SUL}_{\max }$ measurements, as these are more susceptible to noise. Other aspects of the protocol like tracer uptake time, use of furosemide and reconstruction settings were not altered. Although, stringent protocol adherence was aimed for, four patients violated the $\pm 10 \%$ variation in administered dose and \pm 5 min variation in uptake time. As this was not an exclusion criterion and might also occur in clinical practice as well, we decided to include these patients. This however did result in an increased repeatability compared to the use of a clean dataset without these violations (Supplemental Figure 2). Still, if centres 
want to perform response assessment with PSMA-PET/CT, it remains important to adhere to the protocol.

For image processing, VOls were used instead of segmenting the entire organs to provide SUL $L_{\text {mean }}$ values. Still, Li et al. found that there was no significant difference in $\mathrm{SUL}_{\text {mean }}$ of an entire segmented liver and a $\mathrm{VOI}$ with a $3 \mathrm{~cm}$ in diameter [27]. In addition, images were not registered to each other before segmentation, the VOls were drawn by one person based on visual concordance of the location in the two $\mathrm{PET} / \mathrm{CT}$ s. We notice that registration is not commonly used in clinical practice, as it might induce registration errors, and that the chosen segmentation approach mimics our clinical routine for response monitoring.

Next, we did not report on $S U L_{\text {mean }}$ repeatability of the prostate tumour, as threshold-based segmentations using for instance $45 \%$ of SUL max $_{\text {m }}$ proved not an appropriate method, especially for tumours with low SUL $\mathrm{max}_{\text {. }}$ In these patients, almost the entire prostate was segmented with this threshold, thus not representing the actual prostate tumour. To our knowledge, no standardized methods are yet published to accurately define the prostate lesion volume based on PSMA PET/CT. Since SUL $L_{\max }$ is more sensitive to image noise, we chose to obtain the $S U L_{\text {peak }}$ for prostate lesions. 


\section{Conclusion}

${ }^{68} \mathrm{Ga}-\mathrm{PSMA}-11$ PET/CT scans are repeatable in primary prostate cancer patients, with a repeatability coefficient of $18 \% \mathrm{SUL}_{\text {peak }}$ in the primary prostate lesions within a 4-week time period. Variations found in the current study for normal tissues (liver, parotid) were comparable to those previously found in the metastatic setting. Based on these results, ${ }^{68} \mathrm{Ga}-\mathrm{PSMA}-11 \mathrm{PET} / \mathrm{CT}$ scans may be used for accurate surveillance and therapy response monitoring. Still, repeatability and distribution is different from ${ }^{18} \mathrm{~F}-\mathrm{FDG}-\mathrm{PET} / \mathrm{CT}$, indicating that EORTC- or PERCIST-criteria for solid tumours may not be suitable in ${ }^{68} \mathrm{Ga}-\mathrm{PSMA}-11$.

\section{ACKNOWLEDGEMENTS}

We want to thank L. Rooze-Kronenburg, C. Vroonland, M. Kieft and D. Huizing for the help with recruiting and planning the patients. 
Repeatability Coefficient

New (150MBq) and old (100MBq) aqcuisition protocol

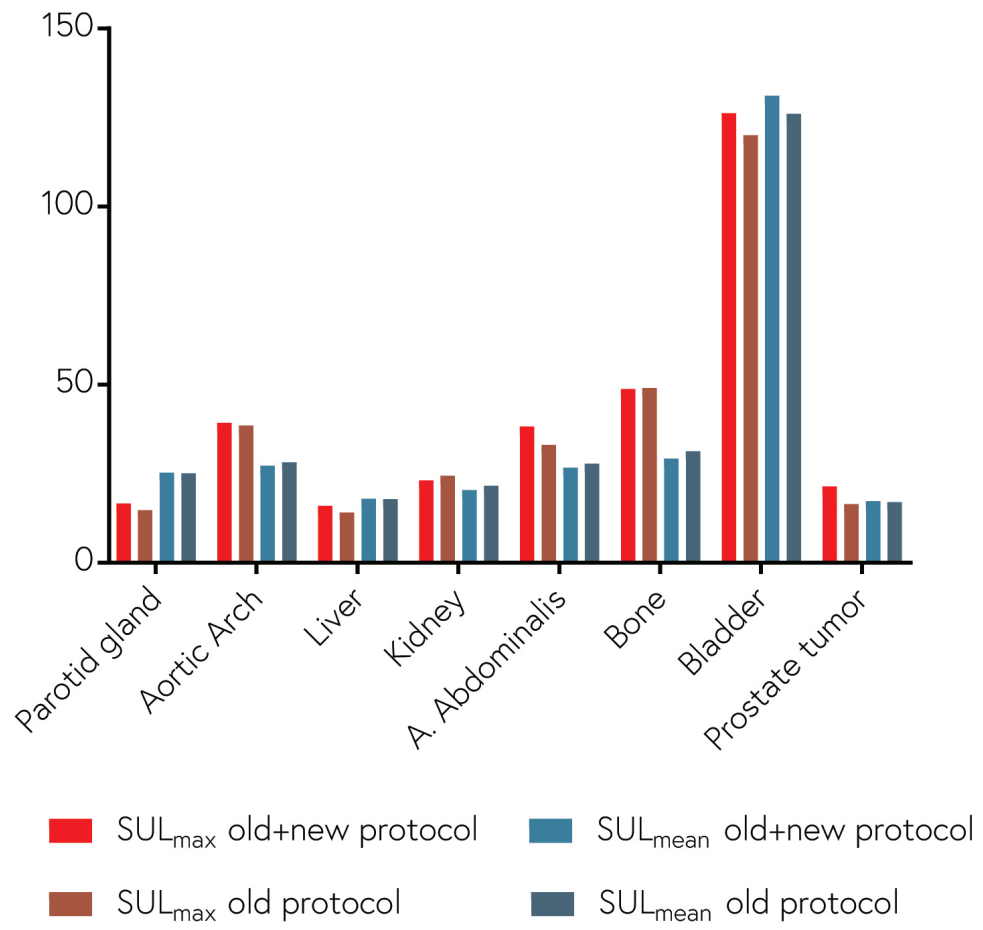

Supplemental Figure 1. Influence of the new protocol (150 MBq \& $4.5 \mathrm{~min} / \mathrm{bp}$ ) on the $\mathrm{RC}_{\text {mean }}$ and $\mathrm{RC} \mathrm{max}_{\text {' }}$ as compared to the old protocol (without protocol violations). Note that the value of the prostate is displayed as SUL peak $_{\text {instead of SUL }}$ mean 


\section{Repeatability Coefficient Influence of aqcuisition protocol violation}

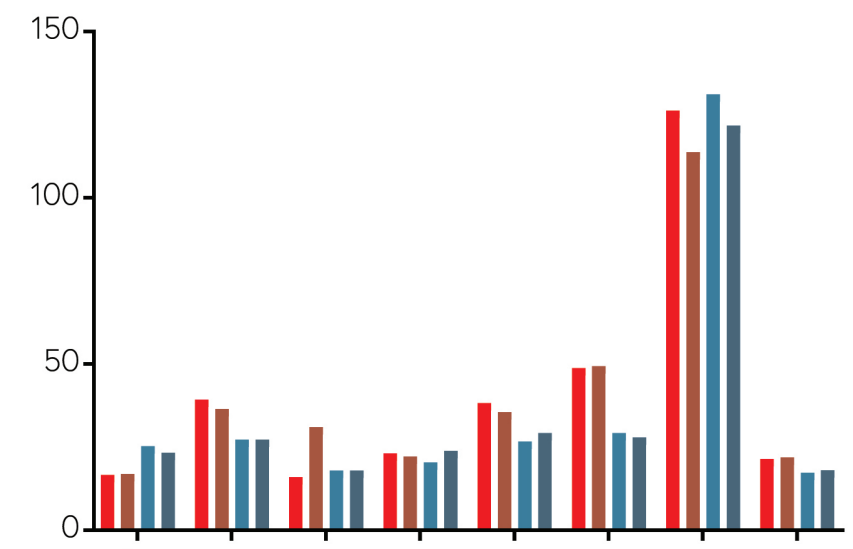

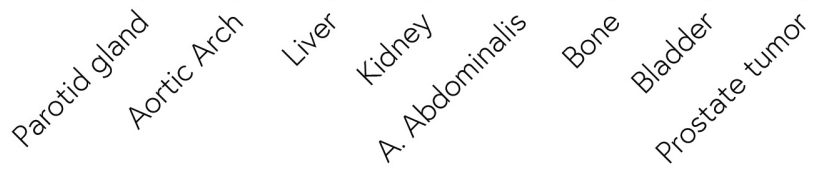

SUL $L_{\text {max }}$ old+new protocol SUL $\quad$ S old+new protocol

SUL $L_{\max }$ with protocol violation $\mathrm{SUL}_{\text {mean }}$ with protocol violation

Supplemental Figure 2. The influence of the protocol violations [ $>10 \%$ difference in administered dose and $>5$ min difference in tracer uptake time] on the $\mathrm{RC}_{\text {mean }}$ and $\mathrm{RC}_{\text {max }}$ were investigated by comparing the RC of the old and new protocol combined to the RC of the old +new protocol with the protocol violations included. Note that the value of the prostate is displayed as $S U L_{\text {peak }}$ instead of $S U L_{\text {mean }}$. Note that the large difference in the SUL ${ }_{\text {max }}$ of the liver is caused by 1 patient, which difference between the scans is very large due to a noisy scan, the RC without this outlier is $18 \%$, more close to the RC without protocol violation [RC 16\%] . 


\section{REFERENCES}

1. Bray F, Ferlay J, Soerjomataram I, et al. Global cancer statistics 2018: GLOBOCAN estimates of incidence and mortality worldwide for 36 cancers in 185 countries. CA. Cancer J Clin. 2018;68:394-424.

2. Mannweiler S, Amersdorfer P, Trajanoski S, et al. Heterogeneity of Prostate-Specific Membrane Antigen (PSMA) Expression in Prostate Carcinoma with Distant Metastasis. Pathol Oncol Res. 2009;15:167-172.

3. Sweat SD, Pacelli A, Murphy GP, et al. Prostate-specific membrane antigen expression is greatest in prostate adenocarcinoma and lymph node metastases. Urology. 1998;52:637640.

4. Maurer T, Weirich G, Schottelius M, et al. Prostate-specific Membrane Antigen-radioguided Surgery for Metastatic Lymph Nodes in Prostate Cancer. Eur Urol. 2015;68:530-534.

5. Perera M, Papa N, Christidis D, et al. Sensitivity, Specificity, and Predictors of Positive ${ }^{68} \mathrm{Ga}-$ Prostate-specific Membrane Antigen Positron Emission Tomography in Advanced Prostate Cancer: A Systematic Review and Meta-analysis. Eur Urol. 2016;70:926-937.

6. Hofman MS, Lawrentschuk N, Francis RJ, et al. Prostate-specific membrane antigen PET-CT in patients with high-risk prostate cancer before curative-intent surgery or radiotherapy (proPSMA): a prospective, randomised, multicentre study. Lancet. 2020;395:1208-1216.

7. Maurer T, Gschwend JE, Rauscher I, et al. Diagnostic efficacy of ${ }^{68} \mathrm{Gallium}-\mathrm{PSMA}$ positron emission tomography compared to conventional imaging for lymph node staging of 130 consecutive patients with intermediate to high risk prostate cancer. J Urol. 2016;195:1436-1442.

8. Herlemann A, Wenter V, Kretschmer A, et al. ${ }^{68}$ Ga-PSMA Positron Emission Tomography/Computed Tomography Provides Accurate Staging of Lymph Node Regions Prior to Lymph Node Dissection in Patients with Prostate Cancer. Eur Urol. 2016;70:553-557.

9. Corfield J, Perera M, Bolton D, et al. ${ }^{68} \mathrm{Ga}$-prostate specific membrane antigen (PSMA) positron emission tomography (PET) for primary staging of high-risk prostate cancer: a systematic review. World J Urol. 2018;36:519-527.

10. Wahl RL, Jacene H, Kasamon Y, et al. From RECIST to PERCIST: Evolving Considerations for PET Response Criteria in Solid Tumours. J Nucl Med. 2009;50:122S-150S.

11. Fendler WP, Eiber M, Beheshti $M$, et al. ${ }^{68}$ Ga-PSMA PET/CT: Joint EANM and SNMMI procedure guideline for prostate cancer imaging: version 1.0. Eur J Nucl Med Mol Imaging. 2017;44:1014-1024.

12. Schmidkonz C, Cordes M, Schmidt D, et al. ${ }^{68}$ Ga-PSMA-11 PET/CT-derived metabolic parameters for determination of whole-body tumour burden and treatment response in prostate cancer. Eur J Nucl Med Mol Imaging. 2018;45:1862-1872.

13. Gupta M, Choudhury PS, Rawal S, et al. Evaluation of RECIST, PERCIST, EORTC, and MDA Criteria for Assessing Treatment Response with ${ }^{68} \mathrm{Ga}$-PSMA PET-CT in Metastatic Prostate Cancer Patient with Biochemical Progression: a Comparative Study. Nucl Med Mol Imaging. 2018;52:420-429.

14. Pollard J, Raman C, Zakharia Y, et al. Quantitative test-retest measurement of ${ }^{68} \mathrm{Ga}-\mathrm{PSMA}-$ HBED-CC (PSMA-11) in tumour and normal tissue. J Nucl Med. 2019; 61:1145-1152.

15. Jansen BHE, Cysouw MCF, Vis AN, et al. Repeatability of Quantitative ${ }^{18} \mathrm{~F}-\mathrm{DCFPyL}$ PET/CT Measurements in Metastatic Prostate Cancer. J Nucl Med. 2020;61:1320-1325 
16. Liu C, Liu T, Zhang N, et al. ${ }^{68} \mathrm{Ga}-\mathrm{PSMA}-617 \mathrm{PET} / \mathrm{CT}$ : a promising new technique for predicting risk stratification and metastatic risk of prostate cancer patients. Eur J Nucl Med Mol Imaging. 2018;45:1852-1861.

17. Gafita A, Calais J, Franz C, et al. Evaluation of SUV normalized by lean body mass (SUL) in ${ }^{68} \mathrm{Ga}-\mathrm{PSMA} 11$ PET/CT: a bi-centric analysis. EJNMMI Res. 2019;9:9-14.

18. James WPT, Waterlow JC. Research on obesity: a report of the DHSS/MRC group. HM Stationery Office; 1976.

19. Jansen BHE, Yaqub M, Voortman J, et al. Simplified Methods for Quantification of ${ }^{18}$ F-DCFPyL Uptake in Patients with Prostate Cancer. J Nucl Med. 2019;60:1730-1735.

20. Bland JM, Altman D. Statistical methods for assessing agreement between two methods of clinical measurement. Lancet. 1986;327:307-310.

21. Lodge MA. Repeatability of SUV in Oncologic ${ }^{18}$ F-FDG PET. J. Nucl. Med. 2017;58:523-532.

22. Ferreira G, Iravani A, Hofman MS, et al. Intra-individual comparison of ${ }^{68} \mathrm{Ga}-\mathrm{PSMA}-11$ and ${ }^{18}$ F-DCFPyL normal-organ biodistribution. Cancer Imaging. 2019;19:23.

23. Giesel FL, Hadaschik B, Cardinale J, et al. F-18 labelled PSMA-1007: biodistribution, radiation dosimetry and histopathological validation of tumour lesions in prostate cancer patients. Eur J Nucl Med Mol Imaging. 2017;44:678-688.

24. O'Keefe DS, Bacich DJ, Huang SS, et al. A Perspective on the Evolving Story of PSMA Biology, PSMA-Based Imaging, and Endoradiotherapeutic Strategies. J Nucl Med. 2018;59:1007-1013.

25. Kabasakal L, Demirci E, Ocak M, et al. Evaluation of PSMA PET/CT imaging using a ${ }^{68}$ GaHBED-CC ligand in patients with prostate cancer and the value of early pelvic imaging. Nucl Med Commun. 2015;36:582-587.

26. Afshar-Oromieh A, Sattler LP, Mier W, et al. The Clinical Impact of Additional Late PET/CT Imaging with ${ }^{68} \mathrm{Ga}-\mathrm{PSM} \mathrm{M}-11(\mathrm{HBED}-\mathrm{CC})$ in the Diagnosis of Prostate Cancer. J Nucl Med. 2017;58:750-755.

27. Afshar-Oromieh A, Hetzheim H, Kübler W, et al. Radiation dosimetry of ${ }^{68}$ Ga-PSMA-11(HBEDCC) and preliminary evaluation of optimal imaging timing. Eur. J Nucl Med Mol Imaging. 2016:43:1611-1620.

28. Li X, Rowe SP, Leal JP, et al. Semiquantitative Parameters in PSMA-Targeted PET Imaging with ${ }^{18 F-D C F P y L: ~ V a r i a b i l i t y ~ i n ~ N o r m a l-O r g a n ~ U p t a k e . ~ J ~ N u c l ~ M e d . ~ 2017 ; 58: 942-946 . ~}$

29. Boellaard R, Delgado-Bolton R, Oyen WJG, et al. FDG PET/CT: EANM procedure guidelines for tumour imaging: version 2.0. Eur J Nucl Med Mol Imaging. 2014;42:328-354.

30. Lückerath K, Stuparu AD, Wei L, et al. Detection Threshold and Reproducibility of ${ }^{68} \mathrm{Ga}-\mathrm{PS}-$ MA11 PET/CT in a Mouse Model of Prostate Cancer. J Nucl Med. 2018;59:1392-1397.

31. Sahakyan K, Li X, Lodge MA, et al. Semiquantitative Parameters in PSMA-Targeted PET Imaging with $\left[{ }^{18} \mathrm{~F}\right] \mathrm{DCFPyL}$ : Intrapatient and Interpatient Variability of Normal Organ Uptake. Mol Imaging Biol. 2020;22:181-189.

32. Beauregard JM, Hofman MS, Kong G, et al. The tumour sink effect on the biodistribution of ${ }^{68} \mathrm{Ga}$-DOTA-octreotate: implications for peptide receptor radionuclide therapy. Eur J Nucl Med Mol Imaging. 2012;39:50-56.

33. Evans MJ, Smith-Jones PM, Wongvipat J, et al. Noninvasive measurement of androgen receptor signaling with a positron-emitting radiopharmaceutical that targets prostate-specific 
membrane antigen. Proc Natl Acad Sci. 2011;108:9578-9582.

34. Meller B, Bremmer F, Sahlmann CO, et al. Alterations in androgen deprivation enhanced prostate-specific membrane antigen (PSMA) expression in prostate cancer cells as a target for diagnostics and therapy. EJNMMI Res. 2015;5:66.

35. Emmett L, Yin C, Crumbaker M, et al. Rapid Modulation of PSMA Expression by Androgen Deprivation: Serial ${ }^{68} \mathrm{Ga}$-PSMA-11 PET in Men with Hormone-Sensitive and Castrate-Resistant Prostate Cancer Commencing Androgen Blockade. J Nucl Med. 2019;60:950-954.

36. Wright $G L$, Haley $C$, Beckett $M L$, et al. Expression of prostate-specific membrane antigen in normal, benign, and malignant prostate tissues. Urol Oncol Semin Orig Investig. 1995;1:1828.

37. Hillier SM, Kern AM, Maresca KP, et al. ${ }^{123}$ I-MIP-1072, a Small-Molecule Inhibitor of Prostate-Specific Membrane Antigen, Is Effective at Monitoring Tumour Response to Taxane Therapy. J Nucl Med. 2011;52:1087-1093 


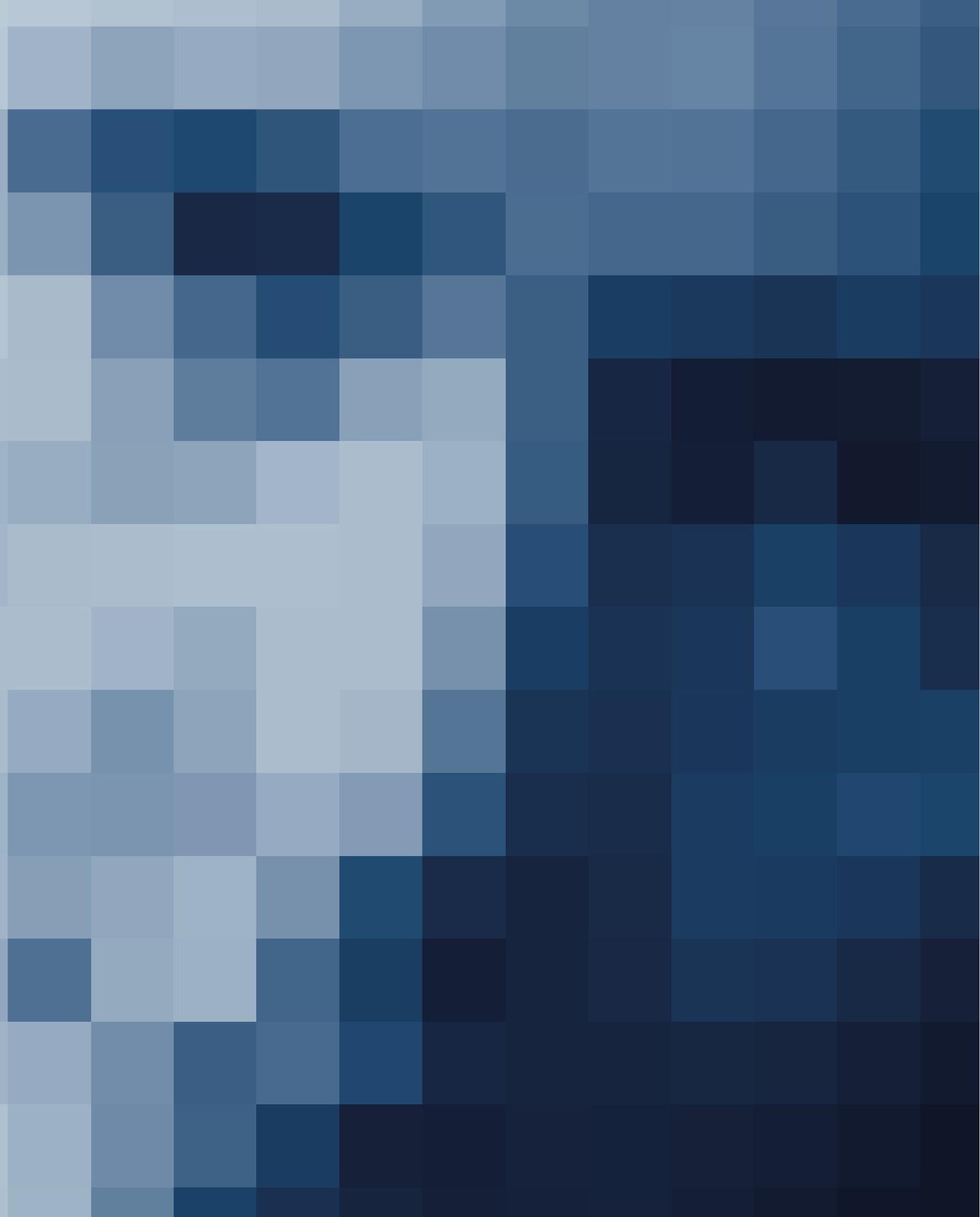




\section{CHAPTER 5}

\section{EARLY DIFFERENCES IN DYNAMIC UPTAKE OF ${ }^{68}$ GA-PSMA-11 IN PRIMARY PROSTATE CANCER:}

ATEST-RETEST STUDY

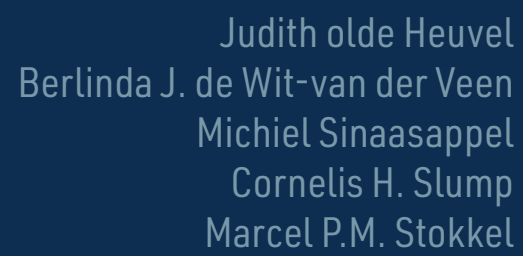

PLOS ONE 2021 16(2): e0246394 


\section{Abstract}

\section{INTRODUCTION}

Dynamic PET/CT allows visualization of pharmacokinetics over the time, in contrast to static whole body PET/CT. The objective of this study was to assess ${ }^{68} \mathrm{Ga}-\mathrm{PSMA}-11$ uptake in pathological lesions and benign tissue, within 30 minutes after injection in primary prostate cancer ( $\mathrm{PC}$ a) patients in test-retest setting.

\section{Methods}

Five patients, with biopsy proven PCa, were scanned dynamically in list mode for 30 minutes on a digital PET/CT scanner directly after an intravenous bolus injection of $100 \mathrm{MBq}{ }^{68} \mathrm{Ga}-\mathrm{PSMA}-11$. Approximately 45 minutes after injection a static whole body scan was acquired, followed by an one bed position scan of the pelvic region. The scans were repeated approximately four weeks later, without any intervention in between. Semi-quantitative assessment was performed using regions-of-interest in the prostate tumour, bladder, gluteal muscle and iliac artery. Time-activity curves were extracted from the counts in these regions and the intra-patient variability between both scans was assessed.

\section{Results}

The uptake of the iliac artery and gluteal muscle reached a plateau after 5 and 3 minutes, respectively. The population fell apart in two groups with respect to tumour uptake: in some patients the tumour uptake reached a plateau after 5 minutes, whereas in other patients the uptake kept increasing, which correlated with larger tumour volumes on PET/CT scan. Median intra-patient variation between both scans was $12.2 \%$ for artery, $9.7 \%$ for tumour, $32.7 \%$ for the bladder and $14.1 \%$ for the gluteal muscle.

\section{CONCLUSION}

Dynamic ${ }^{68} \mathrm{Ga}$-PSMA-11 PET/CT scans, with a time interval of four weeks, are reproducible with a $10 \%$ variation in uptake in the primary prostate tumour. An uptake plateau was reached for the iliac artery and gluteal muscle within 5 minutes post-injection. A larger tumour volume seems to be related to continued tumour uptake. This information might be relevant for both response monitoring and PSMA-based radionuclide therapies. 


\section{Introduction}

$\mathrm{PET} / \mathrm{CT}$ imaging is increasingly used for targeted diagnostic imaging in prostate cancer ( $\mathrm{PC}$ ) , since radiopharmaceuticals labelled to the prostate-specific membrane antigen (PSMA) were introduced 5 years ago [1-6]. The first studies with PSMA-labelled radiotracers from the group of Haberkorn and Eder mainly focused on its' clinical applicability in recurrent PCa. These studies suggested that a 60 minutes tracer uptake is optimal for good visual tumour-to-background contrast in ${ }^{68} \mathrm{Ga}$-PSMA-11 PET/CT imaging [7-9]. The current EANM/SNMMI procedure guideline for PSMA-PET/CT also adapted this uptake time (acceptable range 50-100 minutes), with the remark that late imaging up to 3-4 hours after injection may increase sensitivity for lesion detection. Though uptake-times longer than 100 minutes have reduced count rate (especially relevant for ${ }^{68} \mathrm{Ga}$ ), late imaging may help better identify lesions close to ureters or in patients with low PSMA-expression or PSA levels $[9,10]$.

Nowadays, PSMA-PET/CT not only plays an eminent role in localizing recurrent PCa [11], but also in the diagnosis and staging of primary PCa, especially for high risk patients [12]. Intense bladder accumulation present 45-60 minutes post-injection is known to hamper the visual assessment of the primary tumour, especially on the basal side of the prostate $[13,14]$. Furosemide can be administered after PSMA-ligand injection to increase renal clearance, and hence, reduce tracer accumulation in the urinary system. Early time-point imaging of the pelvic area on the other hand may also reduce the effects of urine accumulation. Multiple studies have reported on dynamic or multi time-point ${ }^{68} \mathrm{Ga}-\mathrm{PSMA}-11$ imaging in primary and recurrent $\mathrm{PCa}$, to evaluate the effectiveness in primary PCa (see supplemental Table 1 for details) [9,10,15-20]. In this setting, 'true' dynamic PET/CT (dPET/CT) refers to visualization of a limited field of view (FoV) with high temporal sampling (typical $<5$ minutes), while multi time-point imaging revers to static acquisitions of a larger FoV with a lower temporal sampling (typical $>5$ minutes). Both imaging approaches provide temporal data which may also hold valuable biological information for lesion characterization.

Yet, limited intra-prostatic imaging data is currently available regarding the distribution phase of ${ }^{68} \mathrm{Ga}-\mathrm{PSMA}-11$ (e.g., the first 30 minutes post-injection). The repeatability of visual aspects and quantitative measures describing both the temporal uptake profile and spatial intra-prostatic distribution pattern are currently unknown, but is relevant when applying early time-point PET for visualization of the basal 
prostate. Additionally, this information is needed for our research regarding intraoperative ${ }^{68} \mathrm{Ga}$-PSMA-11 Cerenkov imaging, where early dynamics might gain insight in the timing of intraoperative injection (NL8256). Next, if considered repeatable, preoperative PET imaging can be used as guidance for intraoperative injection [21]. So, the objective of this study was to assess intra-prostatic ${ }^{68} \mathrm{Ga}-\mathrm{PSMA}-11$ uptake during the first 30 minutes after injection in patients with primary PCa in test-retest setting. 


\section{Methods}

\section{STUDY POPULATION}

This prospective observational cohort study was approved by the Antoni van Leeuwenhoek medical ethics committee (NL8263) and all patients signed written informed consent. No sample size calculation was performed, as the study has only a explorative and descriptive nature, additionally this dynamic imaging sub study was part of a larger clinical trial. Patients were selected at the multidisciplinary team meeting between December 2018 and February 2019 in the Netherlands Cancer Institute - Antoni van Leeuwenhoek. Eligible patients were aged 18 years or older, had histologically confirmed PCa (biopsy proven) and fulfilled one of the three local criteria for the ${ }^{68} \mathrm{Ga}$-PSMA-11 PET/CT scan ( $\geq$ cT3, Gleason score $\geq 4+3=7$, or PSA $\geq 20$ $\mathrm{ng} / \mathrm{mL})$. Six patients were recruited by nurse practitioners or the researcher (JoH) and the study took place in the Netherlands Cancer Institute - Antoni van Leeuwenhoek. Patients were excluded if no ${ }^{68} \mathrm{Ga}-\mathrm{PSMA}-11$ expression was visible on the first PET-scan or when treatment was started in between the two scans.

\section{PET/CTIMAGE ACQUISITION}

Both dynamic and static ${ }^{68} \mathrm{Ga}-\mathrm{PSMA}-11$ PET/CT scans were acquired twice on a digital PET/CT system (Vereos, Philips, Best, the Netherlands) with a four-week interval. The ${ }^{68} \mathrm{Ga}$-Glu-urea-Lys(Ahx)-HBED-CC ( $\left.{ }^{68} \mathrm{Ga}-P S M A-11\right)$ (Scintomics, Fürstenfeldbruck, Germany) was produced as described previously [22].

Patients were not required to fast prior to the scans, and in contrast to EANM guidelines, no furosemide was administered prior to dynamic imaging so not to induce the urge to void during the scan. A dPET/CT scan was acquired in list mode 30 seconds before an intravenous bolus injection $(10 \mathrm{~mL})$ of $100 \mathrm{MBq}$ of ${ }^{68} \mathrm{Ga}-\mathrm{PS}$ MA-11 according to local clinical protocol, until 30 minutes post-injection (p.i.). The acquisition was performed from one bed position $(16.4 \mathrm{~cm})$ with the prostate in the central FoV. A low-dose CT-scan (with 120 kV, 30 mAs with dose modulation, spiral acquisition, reconstruction in $2 \mathrm{~mm}$ slices, and scan range from proximal femora to iliac crest) was made before tracer injection for attenuation correction and anatomic localization [15]. Dynamic data was binned into 30 frames of 1 minute for quantitative evaluation and into 6 frames of 5 minutes for visual assessment. OSEM image reconstruction was performed with isotropic voxel size of $4 \mathrm{~mm}$ at 3 iterations and 
15 subsets without filters or PSF correction, while applying all usual corrections such as for decay, scatter, attenuation, randoms and dead-time.

Subsequently, a static whole body PET/CT scan was performed according to local clinical protocol 45 minutes p.i. and reconstructed with voxel size of $4 \mathrm{~mm}$ (3 iterations, 15 subsets). CT settings were equal to the dynamic settings, but the scan range was from proximal femora to skull base. Subsequently, an additional acquisition of one bed position around the prostate was acquired ( 70 minutes p.i.). The second PET/CT acquisition session was scheduled four weeks later according to the same protocol with an allowed deviation of $<10 \%$ in administered activity.

\section{IMAGE ANALYSIS}

Semi-quantitative evaluation of the dPET data was performed with MATLAB R2017b (The MathWorks, Natick, US, 2017). Regions of interest (ROIs) were manually drawn in the prostate tumour, bladder, gluteal muscle and common iliac artery, and should have a diameter of at least $15 \mathrm{~mm}\left(1.8 \mathrm{~cm}^{3}\right)$ to reduce partial volume effects. The prostate tumour was manually segmented, based on the location and lesion size as displayed on the T2-weighted MRI scan. The arterial ROI (diameter $10 \mathrm{~mm}$ ) was placed in the

102 first frame over 5 consecutive slices and propagated to the subsequent frames. The ROIs for the tumour and gluteal muscle were placed in the last time frame and back propagated to prior frames. Time-activity curves (TACs) were generated for the entire dynamic time range. An image derived input function from the common iliac artery was used to describe the distribution of ${ }^{68} \mathrm{Ga}-\mathrm{PSMA}-11$ in the blood pool, as described in [23]. For the second $\mathrm{dPET} / \mathrm{CT}$, all ROls were redefined and not copied from the previous scan. The volume of the prostate lesion was assessed on the static ${ }^{68} \mathrm{Ga}-\mathrm{PS}$ MA-11 PET/CT scan, using 3D slicer v4.10.0 (slicer.org) to segment the tumour using the PET segmentation extension. This is based on the optimal surface segmentation approach [24], and the segmentation could be manually refined, again also keeping in mind the location and size of the lesion on MRI.

Tracer uptake was defined as either lean body mass (LBM) corrected standardized uptake value $\left(S U L_{\text {mean }}\right.$ ) or tumour-to-blood ratio (TBR). The intra-patient variation was expressed as percentage change and the inter-patient variation is expressed as the Coefficient of Variation (CoV). An uptake 'plateau' was defined as the time where the slope of the uptake is close to zero with a maximum of 0.2. The start of bladder filling was defined as the time where the uptake in the bladder is twice the uptake of the gluteal muscle (background). 


\section{Results}

Six patients were enrolled in this prospective observational cohort study. One patient was excluded for analysis, since this patient had a PSMA-negative tumour. The median time between two scans was 24 days (range 22-28 days). The median net injected activity was 105.9 MBq (range 80.7-108.4 MBq) for the first and 102.5 MBq (range 96.23-106.46 MBq) for the second scan. Further demographics are shown in Table 1.

Table 1. Demographics of the 5 patients included in this study. Data is shown as absolute number [percentage] or as mean [interval).

\begin{tabular}{|c|c|c|c|c|c|}
\hline Patient & 1 & 2 & 3 & 4 & 5 \\
\hline Age (years) & 70 & 73 & 67 & 73 & 76 \\
\hline Gleason score biopsy & $5+4=9$ & $3+4=7$ & $4+3=7$ & $4+3=7$ & $4+3=7$ \\
\hline Gleason score post RALP & $5+4=9$ & $4+4=8$ & x & $3+4=7$ & $4+3=7$ \\
\hline iPSA (ng/mL) & 6.5 & 8.2 & 60 & 6.9 & 16.4 \\
\hline TNM & cT2NOMO & cT3bNOMO & cT3aN1MO & cT1cNOMO & cT1cNOMO \\
\hline Prostate volume on MRI (cc) & 70 & 69 & 50 & 54 & 46 \\
\hline Lesion size on MRI (mm) & $17 \times 8$ & $\begin{array}{c}34 \times 44 \times 28(L) \\
11 \times 11(S)\end{array}$ & $41 \times 15 \times 41$ & $9 \times 5 \times 5$ & $\begin{array}{l}\text { Whole pros- } \\
\text { tate }\end{array}$ \\
\hline Dosage scan 1 / scan 2 (MBq) & $106.5 / 83.2$ & $108.4 / 105.4$ & $98.8 / 80.7$ & $105.1 / 96.2$ & $105.3 / 102.5$ \\
\hline Lesion size on PET (cc) & 4 & $\begin{array}{l}31(\mathrm{~L}) \\
2(\mathrm{~S})\end{array}$ & 48 & 2 & 30 \\
\hline Tumor-blood ratio on PET & 2.0 & 9.1 & 10.8 & 3.09 & 6.3 \\
\hline Therapy & RALP & RALP & exRT & RALP & RALP \\
\hline $\begin{array}{l}\text { PSMA accumulation (stable or } \\
\text { rising) }\end{array}$ & Stable & Rising & Rising & Stable & Rising \\
\hline $\begin{array}{l}A D C \text { value on } M R I\left(\mathrm{~mm}^{2} / \mathrm{s}\right) \\
\text { b-value }(0-200-800)\end{array}$ & 1076 & $\begin{array}{l}793(L) \\
886(S)\end{array}$ & 720 & 826 & 1121 \\
\hline
\end{tabular}

iPSA - Prostate specific antigen, RALP - Robotic assisted Laparoscopic Prostatectomy, exRT - external radiotherapy. Note that patient 2 has a large $(L)$ and a small (S) lesion

\section{REPEATABILITY OF ${ }^{68}$ GA-PSMA-II ACCUMULATION}

A typical example of ${ }^{68} \mathrm{Ga}-\mathrm{PSMA}-11$ accumulation over time in the pelvic region is presented in Figure 1. Evaluation of the dynamic data shows tumour accumulation already within the first 5 minutes in all patients, while mean \pm SD bladder filling was seen at $9 \pm 5$ minutes p.i. (see TACs in Figure 2). The arterial blood input curve demonstrates a steep inflow phase with highest $S U L_{\text {mean }}$ in the first minute $\left(S U L_{\text {mean }}\right.$ 4.3 [range: $3.6-5.4$ ]), whereas after $5 \pm 1$ minutes a plateau was reached in all patients $\left(S U L_{\text {mean }} 1.2\right.$ [0.8-1.8]). In all patients, the uptake plateau in the gluteal muscle 
was reached after $3 \pm 1 \mathrm{~min}$. Continuing accumulation in the prostate tumour was observed in two patients for the full duration (30 minutes) of the scan, while in two patients tumour accumulation plateaued after $5 \pm 1$ min. One patient (no. 2) had two lesions, one which showed increased uptake and one who plateaued (Figure 1\&2).

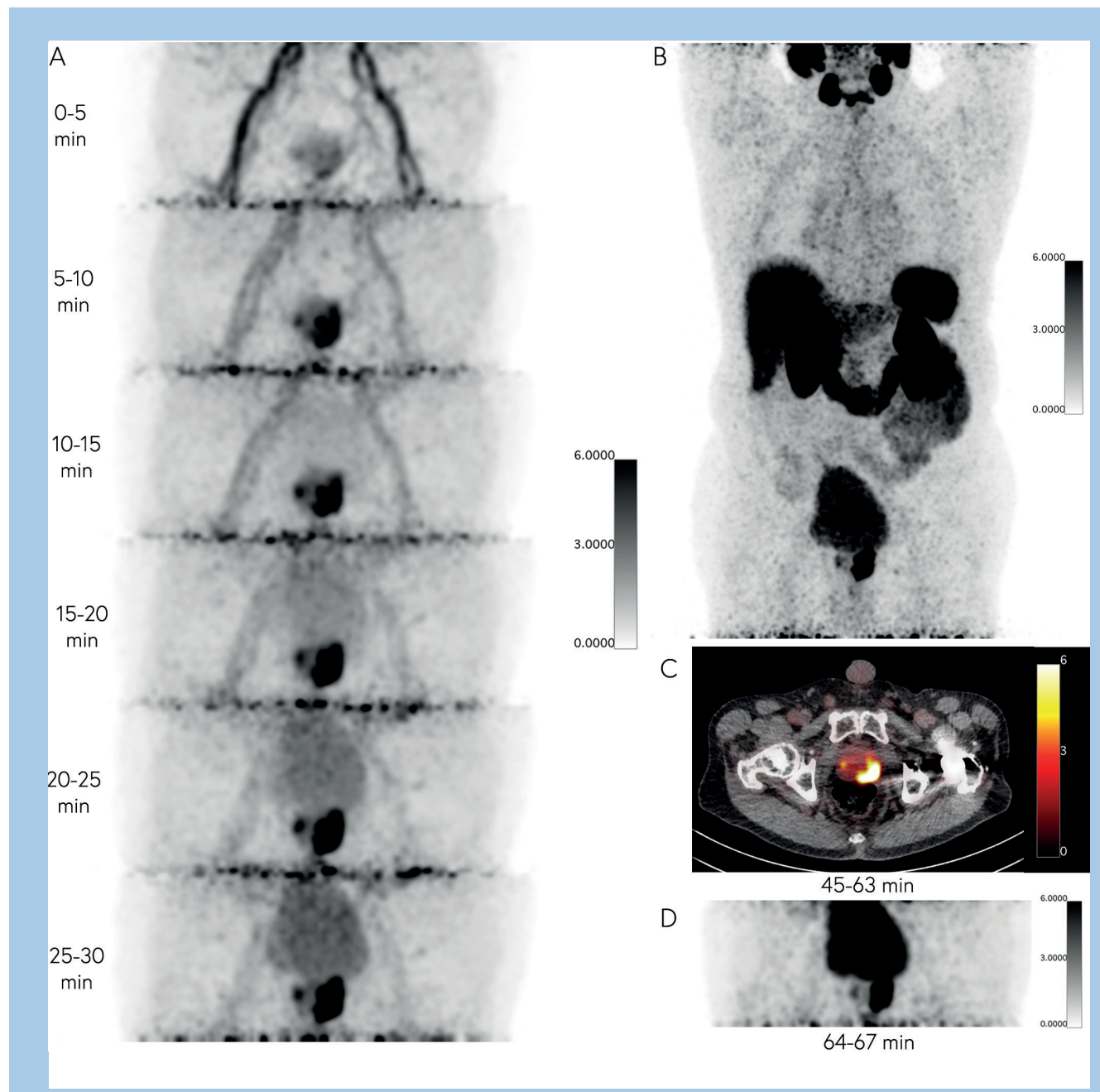

Figure 1. A typical example of dynamic and static ${ }^{68} \mathrm{Ga}-\mathrm{PSMA}-11$ uptake [patient 2]. A] The dynamic PET-data is reconstructed into a time frame of 5 minutes. One can clearly see the prostate tumour uptake in minute 5-10. In the last time frame, the bladder activity is clearly visible. B] Maximum Intensity Profile of the regular clinical static scan in shown. C] PET/CT fusion of the static scan in transverse direction. In D] the delayed static scan of the pelvic region is shown. 

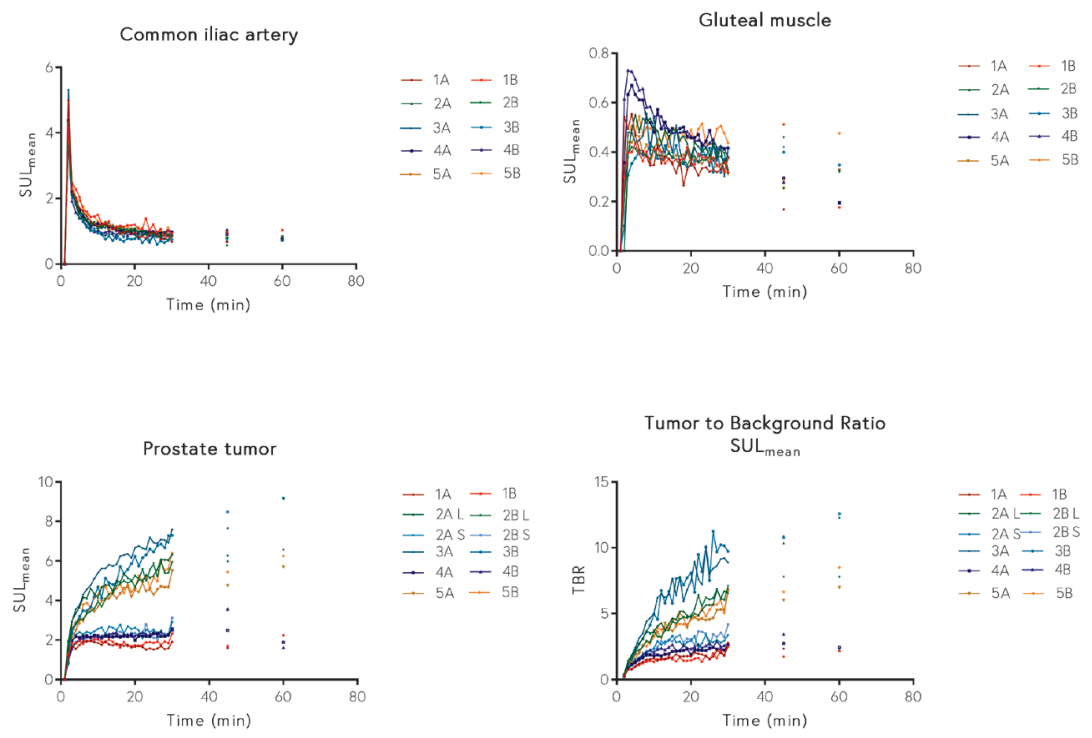

Figure 2. The $S U L_{\text {mean }}$ time activity curve of all patients for the common iliac artery [A], gluteal muscle [B], prostate tumour [C], and the TBR [D]. The TAC of the prostate tumour shows clear deviation in patients $1.2 S, 4$ which become stable in uptake after 5 minutes and the 2L,3,5 which keep increasing. Note that patient 2 has a large $(L)$ and a small $[S$ ) lesion.

${ }^{68} \mathrm{Ga}-\mathrm{PSMA}-11$ uptake at the second $\mathrm{dPET} / \mathrm{CT}$ scan followed the same uptake pattern in the prostate tumour, gluteal muscle and common iliac artery (Figure 2). The intra- and interpatient variation in uptake is provided in Table 2. The largest intra patient variation was observed in bladder TAC (median 32.7\%), while tumour (9.7\%), gluteal (14.1\%) and arterial accumulation (12.2\%) proved to be repeatable over time.

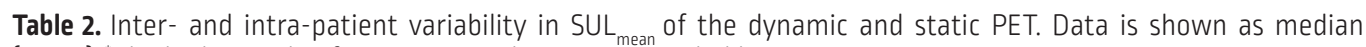
[range]. ${ }^{*}$ The highest value for $\mathrm{SUL}_{\text {mean }}$ on the PET is provided here.

\begin{tabular}{|c|c|c|c|c|c|c|}
\hline \multirow[b]{2}{*}{ Site } & \multicolumn{3}{|c|}{ Dynamic PET } & \multicolumn{3}{|c|}{ Whole body static PET } \\
\hline & $\mathrm{SUL}_{\text {mean }}{ }^{*}$ & Intra-patient (\%) & $\begin{array}{c}\text { Inter-patient } \\
\text { CoV (\%) }\end{array}$ & $S U L_{\text {mean }}$ * & Intra-patient (\%) & $\begin{array}{c}\text { Inter-patient } \\
\text { CoV (\%) }\end{array}$ \\
\hline Artery & $\begin{array}{c}4.3 \\
(3.6-5.3)\end{array}$ & $\begin{array}{c}12.2 \\
(3.4-13.8)\end{array}$ & 12.5 & $\begin{array}{c}0.8 \\
(0.6 / 1.0)\end{array}$ & $\begin{array}{c}14.1 \\
(3.5-45)\end{array}$ & 17.1 \\
\hline Tumor & $\begin{array}{c}5.7 \\
(1.9 / 7.6)\end{array}$ & $\begin{array}{c}9.7 \\
(1.1-16.5)\end{array}$ & 40.5 & $\begin{array}{c}5.1 \\
(1.5 / 8.5)\end{array}$ & $\begin{array}{c}10.7 \\
(4.4-43.1)\end{array}$ & 50.3 \\
\hline Muscle & $\begin{array}{c}0.4 \\
(0.3 / 0.5)\end{array}$ & $\begin{array}{c}14.1 \\
(3.0-18.1)\end{array}$ & 27.0 & $\begin{array}{c}0.3 \\
(0.2 / 0.5)\end{array}$ & $\begin{array}{c}13.2 \\
(5.8-22.9)\end{array}$ & 32.8 \\
\hline Bladder & $\begin{array}{c}8.4 \\
(1.5 / 20.2)\end{array}$ & $\begin{array}{c}32.7 \\
(11.8-100.4)\end{array}$ & 94.1 & $\begin{array}{c}6.4 \\
(2.1 / 26.8)\end{array}$ & $\begin{array}{c}47.3 \\
(5.0-118.8)\end{array}$ & 90.4 \\
\hline
\end{tabular}




\section{TUMOUR PSMA ACCUMULATION}

As mentioned before, not all patients showed an increase in tumour accumulation for the entire duration of the scan. The six lesions of the five patients can be divided into two groups based on the TACs after 5 minutes: one group with stable uptake after 5 minutes $(n=3)$ and the other with an ongoing increase in uptake after 5 minutes $(n=3)$. Note that one patient had two intra-prostatic lesions both with a different uptake pattern. Looking at the first 5 minutes (absorption phase), both groups follow the same pattern. At this absorption phase the SUL varies between the groups, though nog significant (3.5 versus 2.1, p-value 0.1), while the SUL at 20 minutes post-injection differs significantly (5.4 versus $2.1, p$-value 0.003). These patterns were visible both in the test and retest setting.

No evident difference in demographics (Gleason score or initial PSA) was observed between the two groups (Table 1). In addition, also no differences between arterial and gluteal muscle uptake patterns (Figure 3) or tissue diffusion as measured on ADC-MRI was present. The only evident difference was tumour volume, which is about 12 times higher in patients that kept rising (36 cc versus $3 \mathrm{cc}$ ). This difference also occurred in a patient with two different uptake patterns within one scan, with a large and small lesion (31 cc versus 2 cc).

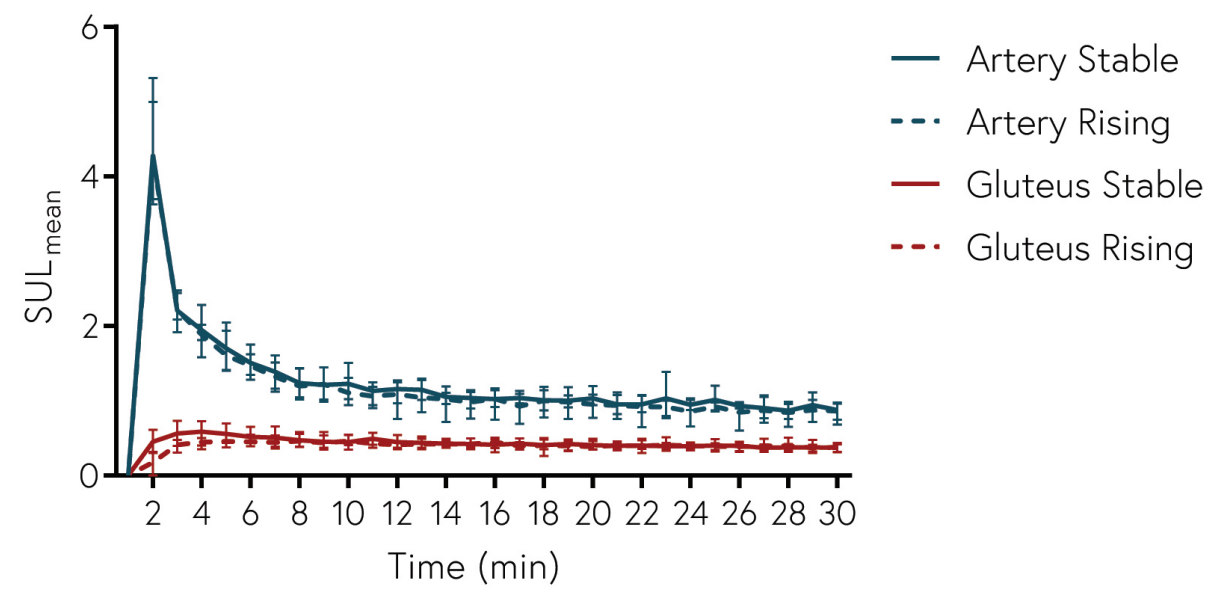

Figure 3. Average time activity curve [trange] of the artery and gluteal muscle, separated into two groups with different uptake patterns in the tumour, in blue the stable group and in red the rising group. 


\section{Discussion}

In the present study, we have evaluated the intra-prostatic ${ }^{68} \mathrm{Ga}-\mathrm{PSMA}-11$ uptake during the first 30 minutes after injection in patients with primary PCa in test-retest setting. The goal was to gain insight into the dynamic behaviour and its repeatability related to intraoperative CLI imaging [21]. The intra patient variation of the TACs was below 15\% for the artery, tumour and gluteal muscle, showing the repeatability of dynamic imaging in primary prostate cancer. The variation of the bladder was higher (32.7\%), which is in line with the expectations based on urinary excretion. This finding is consistent with the repeatability of whole body static PSMA PET/CT scans $45-60$ minutes p.i. [25,26].

With respect to tumour accumulation, this was already visible within the first 5 minutes p.i., which is consistent with the studies by Kabasakal et al. [16], Uprimny et al. [15] and Schmuck et al. [17]. The latter two studies, showed comparable TACs of the gluteal muscle and artery as found in our study, with stable uptake after 5 and 3 minutes respectively. Early imaging might enable better visualization of the prostate tumour, especially at the basal side, as intense urine accumulation in the bladder was seen at $9 \pm 5$ minutes p.i. In contrast, some studies promote late PSMA-PET scans [10,17], which can be especially beneficial for lymph node detection. However, imaging four hours p.i. means that the PET-signal is lower and effects of image noise are higher. Thus, SUL values in late time-point scans should be carefully interpreted. Though early and late time-point imaging both have their pros and cons, there is no clear difference in the number of lesions detected between the two approaches $[16,17]$. So, regarding intraoperative CLI imaging with ${ }^{68} \mathrm{Ga}$-PSMA-11 it was concluded that test-retest accumulation patterns are highly repeatable, and intraoperative detection can commence as early as 5 minutes p.i..

\section{STABLE AND RISING UPTAKE IN PCA}

Visualization of the individual prostate tumour TACs revealed a distinct difference between the patients. Three lesions showed a stable uptake profile after five minutes, whereas three lesions had increasing tumour uptake over time. This effect is not yet described in literature, we think, mainly because previous literature reported the mean TAC of all patients, thus obscuring this effect. The difference in TAC shape might be explained by multiple causes, such as systemic distribution of ${ }^{68} \mathrm{Ga}-\mathrm{PSMA}-11$, tumour biology, technical differences and tumour perfusion, which each will be discussed below. 
After intravenous injection of ${ }^{68} \mathrm{Ga}-\mathrm{PSMA}-11$, it is distributed proportionally through all tissues by the systemic blood flow, so a reduced tumour accumulation might be explained by a limited systemic supply of ${ }^{68} \mathrm{Ga}-\mathrm{PSMA}-11$. When comparing the uptake in the common iliac artery and gluteal muscle, there was no clear difference between both patient groups (arterial SUL $L_{\text {mean }(t=2 \min )}$ stable: 4.3 versus rising: 4.2; gluteal SUL $L_{\text {mean (t=30min) }}$ stable: 0.4 versus rising: 0.4 ). Therefore, it is unlikely that the difference in tumour uptake is explained by a reduced systemic supply of ${ }^{68} \mathrm{Ga}-\mathrm{PS}$ MA-11.

Technical differences, like reconstruction parameters or tracer activity and uptake time, are also known to influence the quantitative values especially in smaller lesions. However, present patients were scanned on a the same scanner, with equal imaging and reconstruction protocols. Furthermore, the measures of each individual patient were repeatable as shown in Table 2. Since tumour volume turned out to be the only relevant parameter, the partial volume effect might explain the difference in uptake [27]. In quantitative PET-imaging, the recovery coefficient is lower for smaller lesions [28], thus implying that the measured uptake is proportional to lesion size. Still, in the current study, the lesion volume did not change over time resulting in a constant underestimation for small lesions, thus not explaining the different shapes of the TACs between the groups.

Perfusion, defined as the rate in which blood flows through the vascular bed of a tissue, can induce heterogeneous accumulation patterns. In large tumours one can presume that not the entire tumour is adequately supplied, despite the upregulated angiogenesis and increased permeability of vessels [29,30]. With dynamic contrast-enhanced MRI perfusion and vascular permeability can be assessed [31], so in future studies this might be considered.

The actual tumour phenotype might also be a factor to clarify the different uptake curves. Since ${ }^{68} \mathrm{Ga}-\mathrm{PSMA}-11$ binds to the PSMA-receptor, the difference might be caused by variations in expression. A plateau in the TAC graphs might indicate total occupancy of the receptors by ${ }^{68} \mathrm{Ga}$-PSMA-11 suggesting that the number of receptors is low in smaller sized tumours. In bigger tumours, on the other hand, there is such a large amount of receptors that the saturated state will not be accomplished within the scanning time of 30 minutes. To test this hypothesis, one should look at the receptor immunohistochemistry staining that matches with the radiolabelled PSMA-ligand. Woythal et al. found that a high SUV ${ }_{\max } 60$ minutes post-injection 
correlates to a tumour lesion with a high immunoreactivity score. Still, this does not mean by definition a bigger lesion, as they did not find a correlation between the size of the lesion and SUV $V_{\max }$ [32]. If indeed a selection of patients show saturation after administration of the ligand, this observation will be relevant for both response monitoring and PSMA-based radionuclide therapies. The amount of PSMA peptide in ${ }^{68} \mathrm{Ga}$-PSMA-11 is typically lower than $10 \mu \mathrm{g}$, and for Lutetium-177 PSMA this is much higher $250 \mu \mathrm{g}$ per cycle. So, for diagnostic imaging this induces a dependency of SUV-measures from ligand concentration and tumour load, whereas for peptide therapies it may result in overdosing of patients with limited tumour load. However, the current population is small, and more research is needed to confirm these findings.

\section{LIMITATIONS}

The current study has some limitations that need to be addressed. First of all, the number of patients is too small to draw definite conclusions, even though the differences in absorption phase and tumour $S U L_{t=20 m i n}$ between the groups seem significant. Still, we think our findings demonstrate an interesting phenomenon, which is worth exploring in the future. Second, the TACs of the first 30 minutes were not continued to the two later static time points, since extrapolating the curve from this single time point will enter too much uncertainty about the uptake pattern. Nevertheless, those standalone points gave valuable information, since tumour uptake was divided in the same groups. As there is still no consensus or standardization in segmentation of primary prostate cancer lesions on PSMA-PET/CT, manual segmentation was used in this study instead of a threshold-based segmentation. Though generally not preferred, a study by Zamboglou et al. showed that manual segmentation outperformed the fixed threshold method in PSMA-PET/CT, when comparing the size of the segmentation to the histopathological size of the lesion [33]. Still, accumulation on PET is inherently prone to partial volume effects and only provides average accumulation over relatively large voxels, thus thereby limiting the ability to assess intra-tumour heterogeneity and multifocality. Next, in this study we did not perform arterial blood sampling, to verify plasma tracer input functions. However, image-based tumour-to-blood ratios were validated as a simplified method to quantify uptake [34] as shown in Figure 2. 


\section{Conclusion}

Dynamic ${ }^{68} \mathrm{Ga}$-PSMA-11 PET/CT scans are reproducible within a 4 week time period. In general, 5 minutes post injection a plateau is reached for the artery, gluteal muscle and for some prostate tumours. There seems to be a deviation after 5 minutes in patients with a large tumour volume where uptake increases over time and stable uptake in patients with a small tumour volume. This information might be beneficial for patient selection for radionuclide therapy with PSMA as target and early timepoint imaging. 
Please rotate the book

for the optimal experience

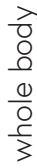

$\stackrel{\infty}{3}$

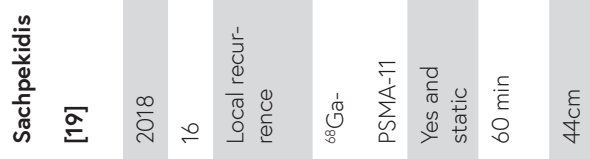

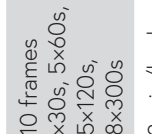

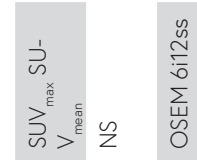

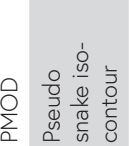

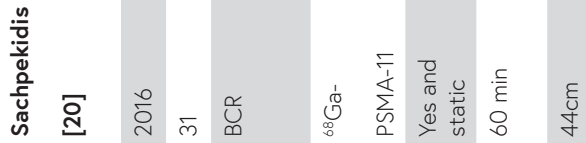

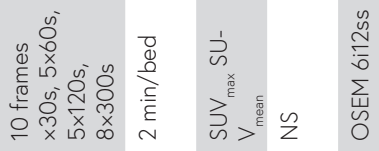

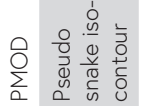

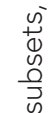

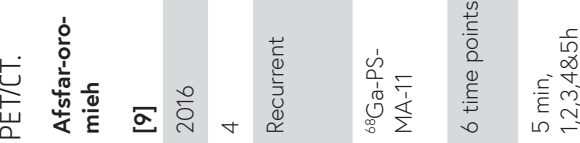

卓

站

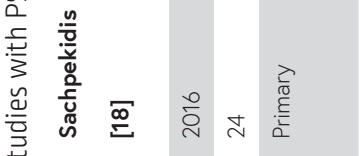

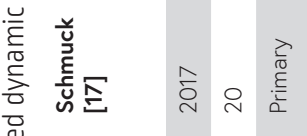

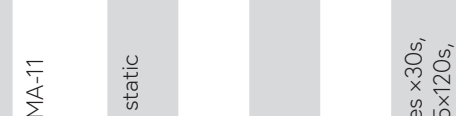

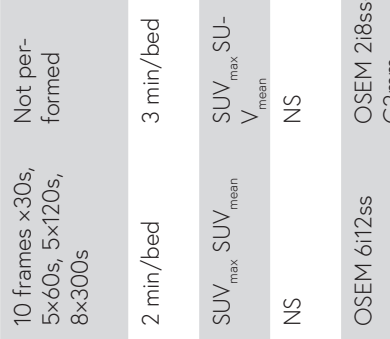

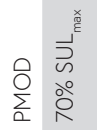

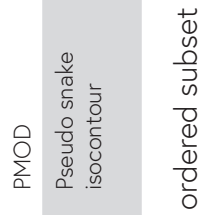

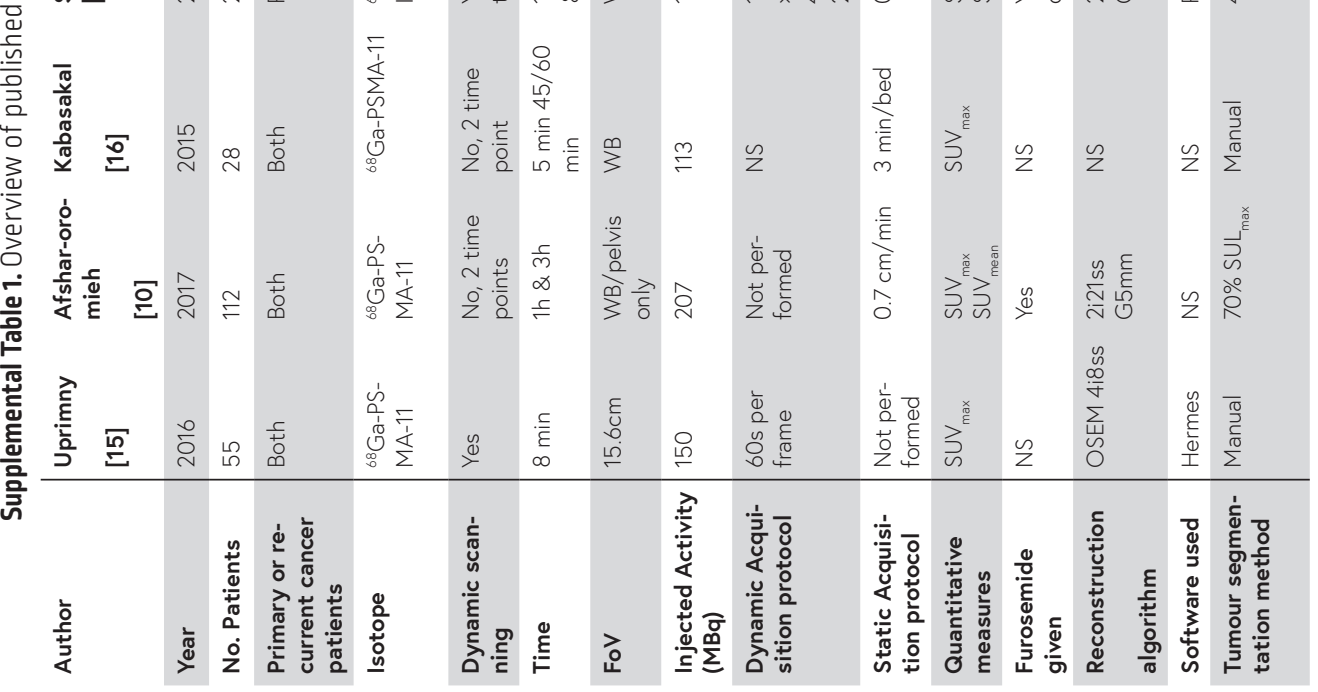

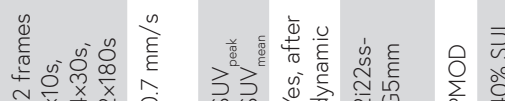

$\sum_{\text {岗 }}^{1}$

$\frac{0}{4}$

in

茫

n

完竞

西 


\section{REFERENCES}

1. Mafeld S, Vasdev N, Patel A, et al. Evolving role of positron emission tomography (PET) in urological malignancy. BJU Int. 2015;116:538-545.

2. Demirkol MO, Acar Ö, Uçar B, et al. Prostate-specific membrane antigen-based imaging in prostate cancer: Impact on clinical decision making process. Prostate. 2015;75:748-757.

3. Eder $M$, Neels $O$, Müller $M$, et al. Novel preclinical and radiopharmaceutical aspects of $\left.{ }^{68} \mathrm{Ga}\right]$ Ga-PSMA-HBED-CC: A new PET tracer for imaging of prostate cancer. Pharmaceuticals. 2014;7:779-796

4. Lütje S, Heskamp S, Cornelissen AS, et al. PSMA ligands for radionuclide imaging and therapy of prostate cancer: Clinical status. Theranostics. 2015;5: 1388-1401.

5. Afshar-Oromieh A, Zechmann CM, Malcher A, et al. Comparison of PET imaging with a ${ }^{68} \mathrm{Ga}-\mathrm{la}-$ belled PSMA ligand and ${ }^{18} \mathrm{~F}$-choline-based PET/CT for the diagnosis of recurrent prostate cancer. Eur J Nucl Med Mol Imaging. 2014;41: 11-20

6. Perera M, Papa N, Christidis D, et al. Sensitivity, Specificity, and Predictors of Positive ${ }^{68} \mathrm{Ga}-$ Prostate-specific Membrane Antigen Positron Emission Tomography in Advanced Prostate Cancer: A Systematic Review and Meta-analysis. Eur Urol. 2016;70: 926-937.

7. Eiber M, Maurer T, Souvatzoglou M, et al. Evaluation of Hybrid ${ }^{68}$ Ga-PSMA Ligand PET/ CT in 248 Patients with Biochemical Recurrence After Radical Prostatectomy. J Nucl Med. 2015;56:668-674.

8. Afshar-Oromieh A, Avtzi E, Giesel FL, et al. The diagnostic value of PET/CT imaging with the ${ }^{68} \mathrm{Ga}$-labelled PSMA ligand HBED-CC in the diagnosis of recurrent prostate cancer. Eur J Nucl Med Mol Imaging. 2014;42:197-209.

9. Afshar-Oromieh A, Hetzheim H, Kübler W, et al. Radiation dosimetry of ${ }^{68} \mathrm{Ga}$-PSMA-11 (HBEDCC) and preliminary evaluation of optimal imaging timing. Eur J Nucl Med Mol Imaging. 2016;43:1611-1620.

10. Afshar-Oromieh A, Sattler LP, Mier W, et al. The Clinical Impact of Additional Late PET/CT Imaging with ${ }^{68} \mathrm{Ga}-\mathrm{PSMA}-11$ (HBED-CC) in the Diagnosis of Prostate Cancer. J Nucl Med. 2017;58:750-755.

11. Evans JD, Jethwa KR, Ost P, et al. Prostate cancer-specific PET radiotracers: A review on the clinical utility in recurrent disease. Pract Radiat Oncol. 2018;8:28-39.

12. Hofman MS, Lawrentschuk N, Francis RJ, et al. Prostate-specific membrane antigen PET-CT in patients with high-risk prostate cancer before curative-intent surgery or radiotherapy (proPSMA): a prospective, randomised, multicentre study. Lancet. 2020;395:1208-1216.

13. Rauscher I, Maurer T, Fendler WP, et al. ${ }^{68} \mathrm{Ga} a \mathrm{PSMA}$ ligand PET/CT in patients with prostate cancer: How we review and report. Cancer Imaging. 2016;16:1-10.

14. Demirci E, Sahin OE, Ocak M, et al. Normal distribution pattern and physiological variants of 68Ga-PSMA-11 PET/CT imaging. Nucl Med Commun. 2016;37:1169-1179.

15. Uprimny C, Kroiss AS, Decristoforo $C$, et al. Early dynamic imaging in ${ }^{68} \mathrm{Ga}-\mathrm{PSMA}-11 \mathrm{PET} / \mathrm{CT}$ allows discrimination of urinary bladder activity and prostate cancer lesions. Eur J Nucl Med Mol Imaging. 2017;44:765-775.

16. Kabasakal L, Demirci E, Ocak M, et al. Evaluation of PSMA PET/CT imaging using a ${ }^{68}$ Ga-HBEDCC ligand in patients with prostate cancer and the value of early pelvic imaging. Nucl Med Commun. 2015;36:582-587.

17. Schmuck S, Mamach M, Wilke F, et al. Multiple time-point ${ }^{68} \mathrm{Ga}-\mathrm{PSMA}$ I\&T PET/CT for charac- 
terization of primary prostate cancer value of early dynamic and delayed imaging. Clin Nucl Med. 2017;42:e286-e293.

18. Sachpekidis C, Kopka K, Eder M, et al. ${ }^{68} \mathrm{Ga}$-PSMA-11 Dynamic PET/CT Imaging in Primary Prostate Cancer. Clin Nucl Med. 2016;41:e473-e479.

19. Sachpekidis C, Pan L, Hadaschik BA, et al. ${ }^{68} \mathrm{Ga} a-P S M A-11$ PET/CT in prostate cancer local recurrence: impact of early images and parametric analysis. Am J Nucl Med Mol Imaging. 2018;8:351-359.

20. Sachpekidis C, Eder M, Kopka K, et al. ${ }^{68} \mathrm{Ga}$-PSMA-11 dynamic PET/CT imaging in biochemical relapse of prostate cancer. Eur J Nucl Med Mol Imaging. 2016;43: 1288-1299.

21. olde Heuvel J, de Wit-van der Veen BJ, van der Poel HG, et al. ${ }^{68} \mathrm{Ga}-\mathrm{PSMA}$ Cerenkov luminescence imaging in primary prostate cancer: first-in-man series. Eur J Nucl Med Mol Imaging. 2020;47:2624-2632.

22. Aalbersberg EA, Geluk-Jonker MM, Young-Mylvaganan T, et al. A Practical Guide for the Production and PET/CT Imaging of ${ }^{68} \mathrm{Ga}$-DOTATATE for Neuroendocrine Tumors in Daily Clinical Practice. J Vis Exp. 2019;146:e59358,

23. Sachpekidis C, Kopka K, Eder M, et al. ${ }^{68} \mathrm{Ga}$-PSMA-11 dynamic PET/CT imaging in primary prostate cancer. Clin Nucl Med. 2016;41: e473-e479.

24. Beichel RR, Van Tol M, Ulrich EJ, et al. Semiautomated segmentation of head and neck cancers in 18F-FDG PET scans: A just-enough-interaction approach. Med Phys. 2016;43: 2948-2964.

25. Jansen BHE, Cysouw MCF, Vis AN, et al. Repeatability of Quantitative ${ }^{18}$ F-DCFPyL PET/CT Measurements in Metastatic Prostate Cancer. J Nucl Med. 2020;61:1320-1325.

26. Pollard J, Raman C, Zakharia Y, et al. Quantitative test-retest measurement of ${ }^{68} \mathrm{Ga}-\mathrm{PSMA}-$ HBED-CC (PSMA-11) in tumor and normal tissue. J Nucl Med. 2019;61:1145-1152.

27. Cysouw MCF, Kramer GM, Schoonmade LJ, et al. Impact of partial-volume correction in oncological PET studies: a systematic review and meta-analysis. Eur J Nucl Med Mol Imaging. 2017:44:2105-2116.

28. Huizing DMV, Koopman D, van Dalen JA, et al. Multicentre quantitative ${ }^{68} \mathrm{Ga}$ PET/CT performance harmonisation. EJNMMI Phys. 2019;6:0-8.

29. Bigler SA, Deering RE, Brawer MK. Comparison of microscopic vascularity in benign and malignant prostate tissue. Hum Pathol. 1993;24:220-226.

30. Nicholson B, Schaefer G, Theodorescu D. Angiogenesis in prostate cancer: Biology and therapeutic opportunities. Cancer Metastasis Rev.2001;20:297-319.

31. Verma S, Turkbey B, Muradyan N et al. Overview of Dynamic Contrast-Enhanced MRI in Prostate Cancer Diagnosis and Management. AJR Am J Roentgenol. 2012;198:1277-88.

32. Woythal N, Arsenic R, Kempkensteffen C, et al. Immunohistochemical Validation of PSMA Expression Measured by $\left({ }^{68}\right) \mathrm{Ga}-\mathrm{PSMA}$ PET/CT in Primary Prostate Cancer. J Nucl Med. 2018;59:238-243.

33. Zamboglou C, Carles M, Fechter $T$, et al. Radiomic features from PSMA PET for non-invasive intraprostatic tumor discrimination and characterization in patients with intermediateand high-risk prostate cancer - A comparison study with histology reference. Theranostics. 2019;9:2595-2605.

34. Jansen BHE, Yaqub M, Voortman J, et al. Simplified Methods for Quantification of ${ }^{18}$ F-DCFPyL Uptake in Patients with Prostate Cancer. J Nucl Med. 2019;60:1730-1735. 



$$
\text { PART III }
$$

\section{INTRAOPERATIVE USE OF ${ }^{68}$ GA-PSMA-11 CERENKOV \\ LUMINESCENCE IMAGING FOR MARGIN ASSESSMENT IN PROSTATE CANCER SURGERY}




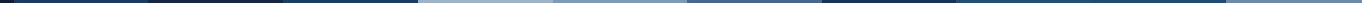




\section{CHAPTER 6}

\section{${ }^{68}$ GA-PSMA CERENKOV LUMINESCENCE IMAGING IN PRIMARY PROSTATE CANCER:}

FIRST-IN-MAN SERIES

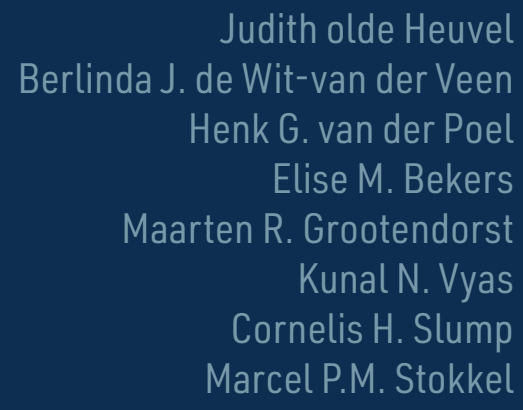

Eur J Nucl Med Mol Imaging. 2020;47:2624-2632 


\section{Abstract}

\section{INTRODUCTION}

Currently, approximately 11-38\% of prostate cancer (PCa) patients undergoing radical prostatectomy have a positive surgical margin (PSM) on histopathology. Cerenkov luminescence imaging (CLI) using gallium-68 prostate-specific membrane antigen ( ${ }^{68} \mathrm{Ga}$-PSMA-11) is a novel technique for intraoperative margin assessment. The aim of this first-in-man study was to investigate the feasibility of intraoperative ${ }^{68} \mathrm{Ga}-\mathrm{PSMA}-11 \mathrm{CLI}$. In this study feasibility was defined as the ability to distinguish between a positive and negative surgical margin, imaging within 45 minutes and low radiation exposure to staff.

\section{Methods}

Six patients were included in this ongoing study. Following perioperative intravenous injection of $\sim 100 \mathrm{MBq}{ }^{68} \mathrm{Ga}-\mathrm{PSMA}-11$, the prostate was excised and immediately imaged ex vivo. Different acquisition protocols were tested, and hotspots on CLI images from the intact prostate were marked for comparison with histopathology.

118 Results

By using an acquisition protocol with 150s exposure time, $8 \times 8$ binning and a $550 \mathrm{~nm}$ shortpass filter, PSMs and negative surgical margins (NSMs) were visually correctly identified on CLI in 3 of the 5 patients. Two patients had a hotspot on CLI from cancer $\leq 0.1 \mathrm{~mm}$ from the excision margin.

\section{CONCLUSION}

Overall the study showed that ${ }^{68} \mathrm{Ga}-\mathrm{PSMA}-11 \mathrm{CLI}$ is a feasible and low-risk technique for intraoperative margin assessment in PCa. The remaining patients in this ongoing study will be used to assess the diagnostic accuracy of the technique. 


\section{Introduction}

Approximately $11-38 \%$ of patients treated with radical prostatectomy have a positive surgical margin (PSM) on final histopathology $[1,2]$. Although conflicting results on the long-term oncological effects on survival have been published, adjuvant local radiotherapy was found to reduce biochemical recurrence rate and is therefore offered to men with PSM $[1,3,4]$. Complete surgical excision is challenging as the surgeon currently depends on visual and tactile appearance to distinguish cancerous from normal tissue. Additional modalities for intraoperative margin assessment can enable more radical excision. NeuroSAFE is a histopathological technique that applies fresh-frozen section evaluation on areas near the neurovascular bundle during surgery. This technique is currently used in several clinics to reduce PSM rates while improving functional outcomes [5-7]. As this technique aims at conserving the neurovascular bundle, rather than actual margin assessment of the prostate surface, only the posterolateral part of the prostate will be sampled. Next, the NeuroSAFE technique is labour-intensive, time consuming ( 45 minutes) and prone to sampling errors as only a small part of the prostate is assessed $[7,8]$.

Other imaging-based technologies to assess margin status are being developed, one of which is Cerenkov luminescence imaging (CLI). Cerenkov radiation is produced when a charged particle (e.g., beta particle from isotopes such as fluorine-18, gallium-68, zirconium-89, yttrium-90, etc.) travels faster than the speed of light trough a dielectric medium such as tissue [9-11]. When the locally polarized medium along the path of that particle returns to its ground state, broad spectrum electromagnetic radiation known as Cerenkov radiation is emitted. Because of the weak light intensity of Cerenkov radiation, it can only be detected by means of highly sensitive optical imaging systems in a dark environment. Roughly one decade ago, $\mathrm{CLI}$ became of interest to the biomedical field [12], after publications that modern sensitive CCD detectors are able to visualize Cerenkov radiation in mice. The initial studies using fluorine-18 and zirconium-89 showed that accurate visualization and quantification of Cerenkov radiation is possible [10-16]. The first clinical study of CLI was published in 2013 using lodine-131 to image the thyroid gland [17], and in 2017 the feasibility of CLI for intraoperative margin assessment was demonstrated in breast cancer [18]. In the latter study patients received a preoperative injection of ${ }^{18} \mathrm{~F}$-Fluorodeoxyglucose $\left({ }^{18} \mathrm{~F}-\mathrm{FDG}\right)$, followed by breast-conserving surgery and intraoperative imaging of excised wide local excision specimens in a dedicated CLI 
system to assess margin status.

Applicability of ${ }^{18} \mathrm{~F}-\mathrm{FDG}$ in prostate cancer ( $\mathrm{PCa}$ ) is unfortunately limited, since there is a low uptake of glucose due to the low rate of glycolysis $[19,20]$. With the introduction of tracers that target the prostate-specific membrane antigen (PSMA), the role of molecular imaging in PCa changed rapidly. Positron emission tomography (PET) using gallium-68 ( $\left.{ }^{68} \mathrm{Ga}\right)$ or ${ }^{18} \mathrm{~F}$-labelled PSMA-ligands are increasingly used for primary staging in high-risk patients, with excellent diagnostic performance [21]. An important aspect for $\mathrm{CLI}$ is the initial positron energy of an isotope; the higher the initial energy, the longer the path length trough tissue, and hence, the higher the Cerenkov-yield. So, in addition to the receptor-targeted accumulation of PSMA-ligands in $\mathrm{PCa}$ lesions, the advantage of ${ }^{68} \mathrm{Ga}$-labelled radiopharmaceuticals for $\mathrm{CLI}$ is the high Cerenkov yield [16]. In our previous study we demonstrated that the Cerenkov yield from ${ }^{68} \mathrm{Ga}$ is approximately 22 times higher compared to ${ }^{18} \mathrm{~F}$, which should enable clinical translation of CLI in PCa [22].

The aim of this first-in-man clinical study was to assess the feasibility of ${ }^{68} \mathrm{Ga}-\mathrm{PS}$ MA-11 CLI for intraoperative margin assessment in PCa. Here clinical feasibility of the technology is defined as the ability to distinguish between a PSM and a negative surgical margin (NSM) by imaging the entire prostate within 45 minutes (typical duration of the NeuroSAFE procedure) and with low radiation exposure to staff. 


\section{Methods}

\section{PATIENT INCLUSION}

Present study was approved by the local medical ethics committee (NL8256). Six high-risk primary PCa patients scheduled for robot-assisted radical prostatectomy (RARP) were enrolled in this on-going trial after written informed consent was obtained. The main inclusion criteria were a tumour lesion $\geq 1.5 \mathrm{~cm}$ on MRI, and a PSMA-positive intra-prostatic lesion on PSMA PET/CT. In all patients CLI images were not used for surgical or histopathological decision making.

\section{SURGERY AND INTRAOPERATIVE CLI}

An intravenous injection of $\sim 100 \mathrm{MBq}{ }^{68} \mathrm{Ga}$-Glu-urea-Lys (Ahx)-HBED-CC (ABX $\mathrm{GmbH}$, Radeberg, Germany) was given immediately after the da Vinci ${ }^{\oplus}$ surgical system was docked. After removal, the prostate was prepared for Cerenkov imaging by rinsing it with sodium chloride to clear any potential radioactive contamination from blood or urine.

Images from six sides of the prostate (left/right, anterior/posterior, basal/apical) were acquired using the LightPath ${ }^{\circledast} \mathrm{CLI}$ system (Lightpoint Medical Ltd, Chesham, UK) (Figure 1). This device consists of a light-tight specimen chamber, and acquires a photographic reference image and a Cerenkov image which are automatically overlaid [23]. To reduce the noise from high energy gamma photons (called gamma strikes), the images are immediately processed by the LightPath ${ }^{\circledR}$ software by applying a Gaussian filter ( $\sigma=3$ pixels) and a median filter ( $3 \times 3$ pixels) and then displayed. In our previous in vitro study, it was found that images with 120 s exposure, $2 \times 2$ binning and no filter were sufficient for detection of clinically relevant ${ }^{68} \mathrm{Ga}$-PSMA-11 activity levels [21]. This imaging protocol formed the basis for the current ex vivo clinical study. To account for potential differences in signal levels that can be expected when converting in vitro results to an ex vivo clinical setting, in the current study images were acquired with different acquisition times $(30,60$,

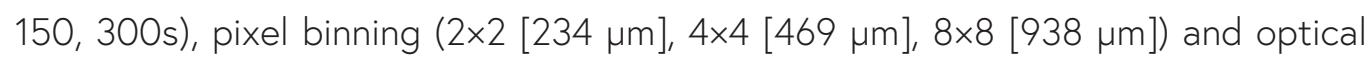
filter (no filter and $550 \mathrm{~nm}$ shortpass filter) with the aim to identify an acquisition protocol that provided sufficient sensitivity within a time-window feasible for intraoperative use ( $<45$ minutes).

If areas of increased signal on CLI (called "hotspots") were visualised on the intact images acquired without an optical filter, the acquisition was repeated with a 550 
$\mathrm{nm}$ shortpass filter. As shorter-wavelength light has a higher attenuation and scattering in tissue, a persistent hotspot on the shortpass-filtered image indicates presence of ${ }^{68} \mathrm{Ga}-\mathrm{PSMA}$-containing cancer cells near the excision margin. Based on visual assessment of the intact images, hotspots were marked with a suture for accurate comparison with histopathology.

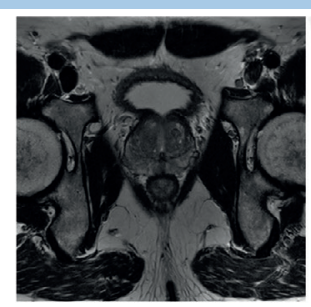

A

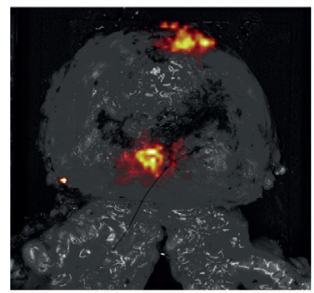

E

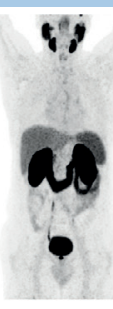

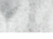

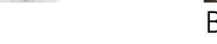

B

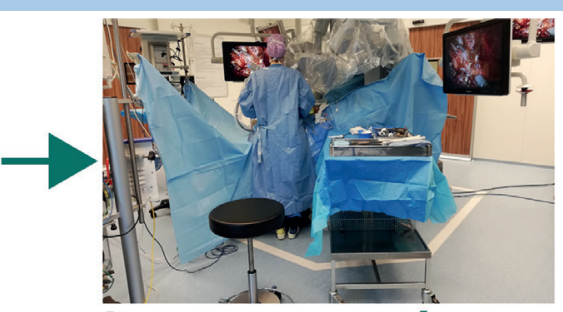

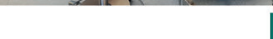

$\downarrow$

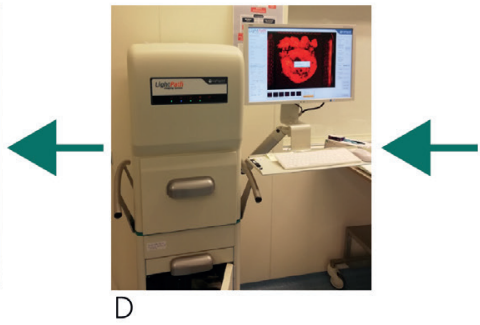

D

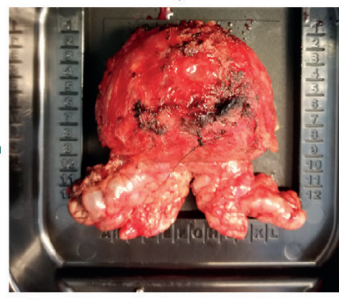

C
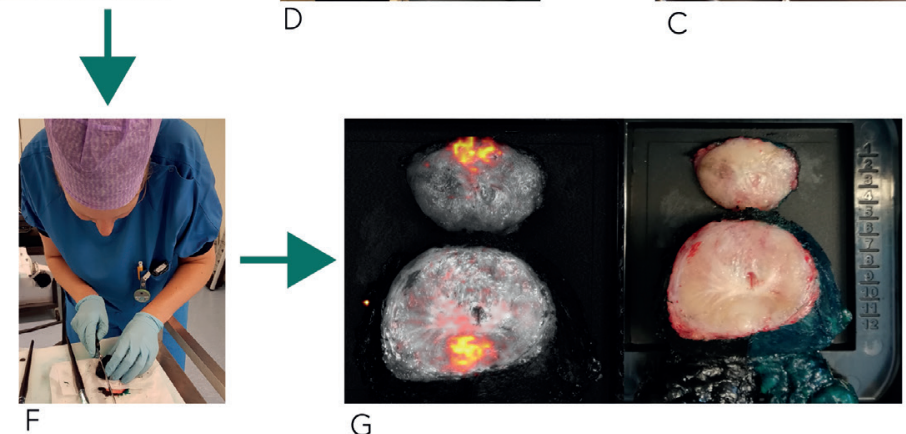

G

Figure 1. Workflow of the current CLI study, (A) A preoperative ${ }^{68} \mathrm{Ga}-\mathrm{PSMA}-11$ PET-CT and MRI scan are acquired 3-4 weeks prior to surgery as per standard of care, based on the result of these images patients are included. (B) During surgery ${ }^{68}$ Ga-PSMA-11 is administered i.v. after the da Vinci surgical system is docked. Radiation dose to all surgical staff is monitored. Note the position of the scrub nurse in close proximity to the patient. Once removed, the prostate is rinsed with $\mathrm{NaCl}$ to clear any radioactive urine and blood that could be present on the surface. (C) The prostate is positioned in a disposable specimen tray. (D) Images of all six sides of the prostate using the different settings are acquired in the CLI device. (E) Unfiltered Cerenkov image of the intact prostate specimen (F) Upon image completion of the intact prostate, the prostate is inked and cleaved $\sim 1 \mathrm{~cm}$ from the apex by a trained person. (G) Image of the cleaved prostate and the corresponding CLI image acquired with the same settings as the intact specimen to confirm tumour uptake and quantify the intensity in benign and cancerous tissue. 
Following CLI of the intact prostate specimen, the surface of the prostate was inked and cleaved $1 \mathrm{~cm}$ from the apex according to standard histopathological protocol. The cleaved prostate was subsequently imaged with CLI to visualize the primary lesion, to confirm presence of ${ }^{68} \mathrm{Ga}-\mathrm{PSMA}-11$ in the primary lesion and to quantify the signal in benign and cancerous tissue. The workflow of the CLI-procedure is displayed in Figure 1.

\section{RADIATION SAFETY MONITORING}

As ${ }^{68} \mathrm{Ga}$-PSMA-11 is not routinely used in the operating room, the radiation exposure to the operating room staff was monitored using electronic personal radiation dosimeters (MGP Instruments DMC 2000; Mirion Technologies, Ltd.). Measurements included the scrub nurse positioned directly next to the patient, the surgeon positioned behind the da Vinci surgical robot at three meter from the patient, and the anaesthetist, periphery nurse and researcher positioned more than one meter away from the patient.

\section{HISTOPATHOLOGY}

After completion of CLI, the specimens were taken to the Pathology department. Histopathological examination was performed as per standard of care by an experienced uropathologist (EMB). A PSM was defined as cancer extending into the inked surface [24].

\section{IMAGE ANALYSIS}

In addition to visual assessment of the intact prostate image to qualify if the margins are positive or negative, explorative quantitative assessment was performed after surgery using MATLAB R2017b (The MathWorks, Natrick, WA). The researcher $(\mathrm{JoH})$ who performed this post-hoc evaluation was blinded to the histopathology results, but had access to the patient's PSMA-PET/CT and MRI. First, the images were post-processed by subtracting a background image (i.e., a CLI image of an empty specimen tray acquired with the same acquisition settings) from the clinical $\mathrm{CL}$ images. This was performed to remove any camera noise in the image as well as the artefact from a defect pixel in the camera (Figure 2). Measurements of the mean radiance (photons $(p) / \mathrm{s} / \mathrm{cm}^{2} / \mathrm{sr}$ ) were performed by manually selecting regions of interest (ROI) on the post-processed images of both the intact and cleaved prostate images around areas showing hotspots (tumour) and in areas with no increased signal (tissue background), and based on the latter ROls the tumour-to-background 
ratios (TBRs) were calculated. The quantitative analysis of the cleaved prostate images was performed with the aim to eventually identify a quantitative threshold for discriminating between a PSM and NSM. To enable comparison of signal levels between images of each patient individually the signals (tissue background and tumour) were corrected for radioactive decay. Additionally, decay corrected radiances were also corrected for injected activity to enable comparison of images between patients (corrected radiance). The process of image analysis is displayed in Figure 2.

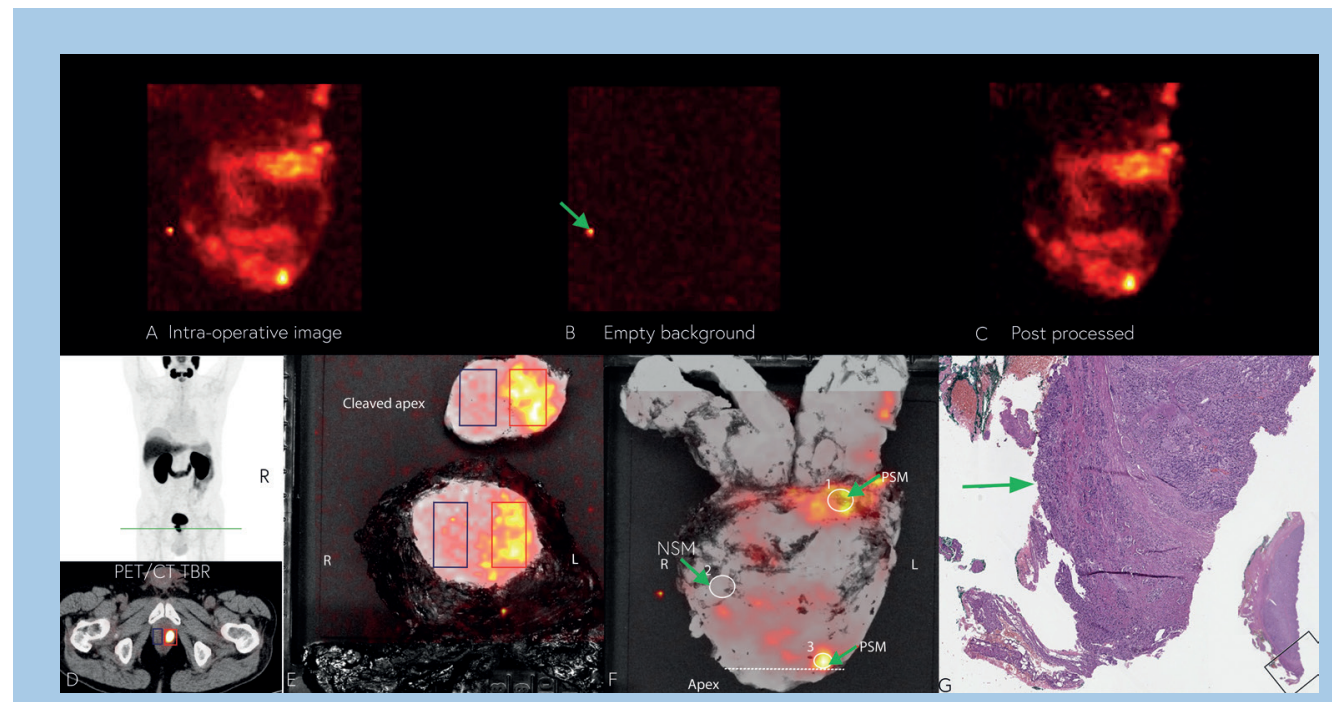

Figure 2. Overview of the image analysis and quantification methods. (A) Intraoperative Cerenkov image of the specimen after median and Gaussian filtering. This image is used for visual identification of a PSMs and NSMs. (B) A Cerenkov image of an empty background. Note the presence of the defective pixel (green arrow). (C) Post-processed Cerenkov image after background subtraction. Note that the background subtraction removes the defective pixel on the post-processed image. (D) The PSMA PET/CT scan. The green line on the Maximum Intensity Profile (MIP) image indicates the location where the prostate is cleaved. The transverse image of the fused PET/CT scan highlights the location of the tumour (red ROI) and benign tissue (blue ROI). (E) Intraoperative CLI image of the prostate from patient 2 after the specimen was cleaved at the apex, showing the tumour (red ROI) and the benign tissue (blue ROI). (F) CLI image of intact prostate specimen from patient 2. Two hotspots, corresponding with a histopathological PSM, can be identified (ROI 1 and $\mathrm{ROI} 3$ ). Areas with no increased signal, corresponding with benign tissue from a NSM, can also be seen (ROI 2). For clarity each $\mathrm{ROI}$ is also highlighted with a green arrow. For this patient the TBR was calculated by dividing the radiance from $\mathrm{ROI} 1$ and $\mathrm{ROI}$ in 2 . The dotted white line represents the location where the prostate was cleaved. G) Histopathology image of the area corresponding with ROI 3 on the intact CLI image. A PSM can be identified (green arrow). 


\section{Results}

Six patients were initially included into the study, but one patient had to be excluded due to unsuccessful ${ }^{68} \mathrm{Ga}$-PSMA-11 labelling on the day of surgery. The mean injected activity was $83 \pm 19 \mathrm{MBq}$, and the mean time between injection and CLI acquisition of the prostate was $73 \pm 14 \mathrm{~min}$. An overview of the patient characteristics, ${ }^{68} \mathrm{Ga}-P S M A-11$ activity, CLI results and histopathological results can be found in Table 1. Two out of five patients had a PSM according to histopathology (patient 1 and 2).

Figure 3 displays images with variable binning, filters and exposure times, which were used to evaluate and optimize the CLI acquisition settings on the intact prostate. An exposure time of 150s was sufficient to detect cancerous lesions on CLI on both the intact prostate specimen (Figure $3 \& 4$ ). Based on the intact prostate images, a 150s exposure was favoured over a 300s acquisition as the TBR for the latter did not sufficiently improve to outweigh the increase in acquisition time (TBR 1.85 vs 1.98). An $8 \times 8$ pixel binning improved the TBR compared to $2 \times 2$ and $4 \times 4$ binning, respectively (TBR 1.85, 1.26, and 1.06). Acquisitions without a filter had a higher TBR (4.33) compared to the ones with filter (TBR 1.85).

Data from the CLI images of the cleaved prostate showed that the tumour had a higher radiance as compared to the benign tissue, both on the filtered (mean TBR: 3.6 \pm 1.4 ) and non-filtered images (mean TBR: 2.1 \pm 0.4 ), Table 1. As expected, the use of a filter decreased the radiance in both tumour and tissue background areas. In patient 3 the tumour could not be identified on the cleaved prostate image, as the tumour was not located in the apex region where the incision was made but in the base.

In the two patients with a PSM (patient 1 and 2), hotspots were identified in corresponding areas on both the non-filtered and the filtered intact prostate image, thus enabling successful identification of PSM on CLI (Figure 4). Three out of five patients had a histopathological NSM. In one of these patients (patient 3), the NSM was correctly identified on $\mathrm{CLI}$ as the hotspot was present on the non-filtered images but not on the filtered image, potentially indicating PSMA-containing cells further away from the resection margin (Figure 4). In the other two patients (patient 4 and 5), a hotspot was identified on the non-filtered and in one patient as well on the filtered $\mathrm{CLI}$ image, and in both cases histopathology found tumour cells $\leq 0.1 \mathrm{~mm}$ from the 
margin. In patient 5, the hotspot was not clearly visible on the filtered intact image, however, the quantitative TBR was high at that location.

The sterile scrub nurse received the highest dose because of their close proximity to the patient: $0.016 \mathrm{mSv}$. The average dose to the anaesthetist, surgeon, periphery nurse, and researcher were substantially lower: $0.001 \mathrm{mSv}, 0.005 \mathrm{mSv}, 0.002 \mathrm{mSv}$ and $0.001 \mathrm{mSv}$, respectively

Table 1. Patient characteristics and CLI and histology results of all 5 patients The displayed radiances are activity and decay corrected. iPSA: initial prostate specimen antigen level. HPath: histopathology TBR: Tumour-to-Background Ratio. ${ }^{\wedge}$ : in this patient the tumour was located in the base of the prostate while the prostate was cleaved $-1 \mathrm{~cm}$ from the apex, thus preventing visualisation of the tumour on CLI. ${ }^{* *}$ : although no histopathological PSM, the location of the hotspot on CLI agreed to a tumour to ink distance of $<0.1 \mathrm{~mm}$ on the histopathology slide. $x$ - No non-filtered image taken. N/A: As there was a NSM in patient 3,4,5 there was no TBR calculated from the intact specimen. Note that patient 2 had a PSM on two locations, therefore the corrected radiance of both hotspots is displayed, as well as the TBR of both locations on the intact prostate. Pre- and postoperative tumour grade are based on the clinical TNM stage [29]: T for primary tumour, $\mathrm{N}$ for nodal stage, $\mathrm{M}$ for metastases. R stands for margin status and $\mathrm{X}$ indicates that the status of a certain characteristic cannot be determined. c for clinical, indicates that the classification is based on clinical parameters. $p$ for pathological, indicates that the classification was performed, in this case on the excised prostate in the pathology lab.

\begin{tabular}{|c|c|c|c|c|c|}
\hline & Patient 1 & Patient 2 & Patient 3 & Patient 4 & Patient 5 \\
\hline Age (y) & 67 & 71 & 58 & 73 & 63 \\
\hline Weight (kg) & 75 & 75 & 106 & 126 & 86 \\
\hline Preoperative Tumour Grade & cT3bNOMO & CT1CNOMO & cT1CNOMO & cT1cNOMO & CT2CNOMO \\
\hline Postoperative Tumour Grade & pT3aNOMx & pT3aNOMx & pT2CNOMx & pT3bN1Mx & рT2NOMx \\
\hline iPSA $(\mu \mathrm{g} / \mathrm{L})$ & 29.9 & 4.4 & 5.3 & 8.3 & 6.4 \\
\hline Gleason Score & $4+4=8$ & $4+4=8$ & $4+4=8$ & $4+5=9$ & $4+9$ \\
\hline Prostate volume MRI (cc) & 47 & 30 & 41 & 76 & 28 \\
\hline $\mathrm{SUV}_{\max }$ on PSMA PET/CT & 24.8 & 31.6 & 4.3 & 49.2 & 21.2 \\
\hline $\begin{array}{l}\text { Injected }{ }^{68} \mathrm{Ga}-\mathrm{PS} M A-11 \text { activity } \\
\text { (MBq) }\end{array}$ & 118 & 68 & 88 & 76 & 65 \\
\hline Activity at start of CLI (MBq) & 45 & 51 & 38 & 42 & 34 \\
\hline Suspected PSM CLI & Yes & Yes & No & Yes & Yes \\
\hline PSM on histopathology & Yes & Yes & No & No & No \\
\hline Location agreement CLI -Hpath & Yes & Yes & $\mathrm{N} / \mathrm{A}$ & Yes ${ }^{\star \star}$ & Yes $^{\star \star}$ \\
\hline $\begin{array}{l}\text { Closeness tumour to surface } \\
(\mathrm{mm})\end{array}$ & 0 & 0 & 3 & 0.1 & 0.1 \\
\hline $\begin{array}{l}\text { Corrected radiance hotspot in- } \\
\text { tact prostate }\left(\mathrm{p} / \mathrm{s} / \mathrm{cm}^{2} / \mathrm{sr}\right) \\
550 \mathrm{~nm} \text { filter } / \mathrm{no} \text { filter }\end{array}$ & $1497 / x$ & $\begin{array}{l}1446 / 16691 \\
1711 / 14533\end{array}$ & $798 / 11139$ & $1617 / 16474$ & $1264 / 12500$ \\
\hline $\begin{array}{l}\text { TBR intact prostate } \\
550 \mathrm{~nm} \text { filter/no filter }\end{array}$ & $3.3 / x$ & $\begin{array}{l}4.0 / 4.7 \\
4.7 / 4.1\end{array}$ & $2.5 / 2.5$ & $3.3 / 2.7$ & $7.7 / 2.5$ \\
\hline $\begin{array}{l}\text { Corrected radiance hotspot } \\
\text { cleaved prostate }\left(\mathrm{p} / \mathrm{s} / \mathrm{cm}^{2} / \mathrm{sr}\right) \\
550 \mathrm{~nm} \text { filter/no filter }\end{array}$ & $2135 / x$ & $1149 / 12554$ & $\wedge$ & $1783 / 16159$ & $2642 / 18430$ \\
\hline $\begin{array}{l}\text { TBR cleaved prostate } \\
550 \mathrm{~nm} \text { filter/no filter }\end{array}$ & $3.1 / x$ & $2.2 / 1.8$ & $\wedge$ & $3.1 / 2.6$ & $5.9 / 1.8$ \\
\hline
\end{tabular}




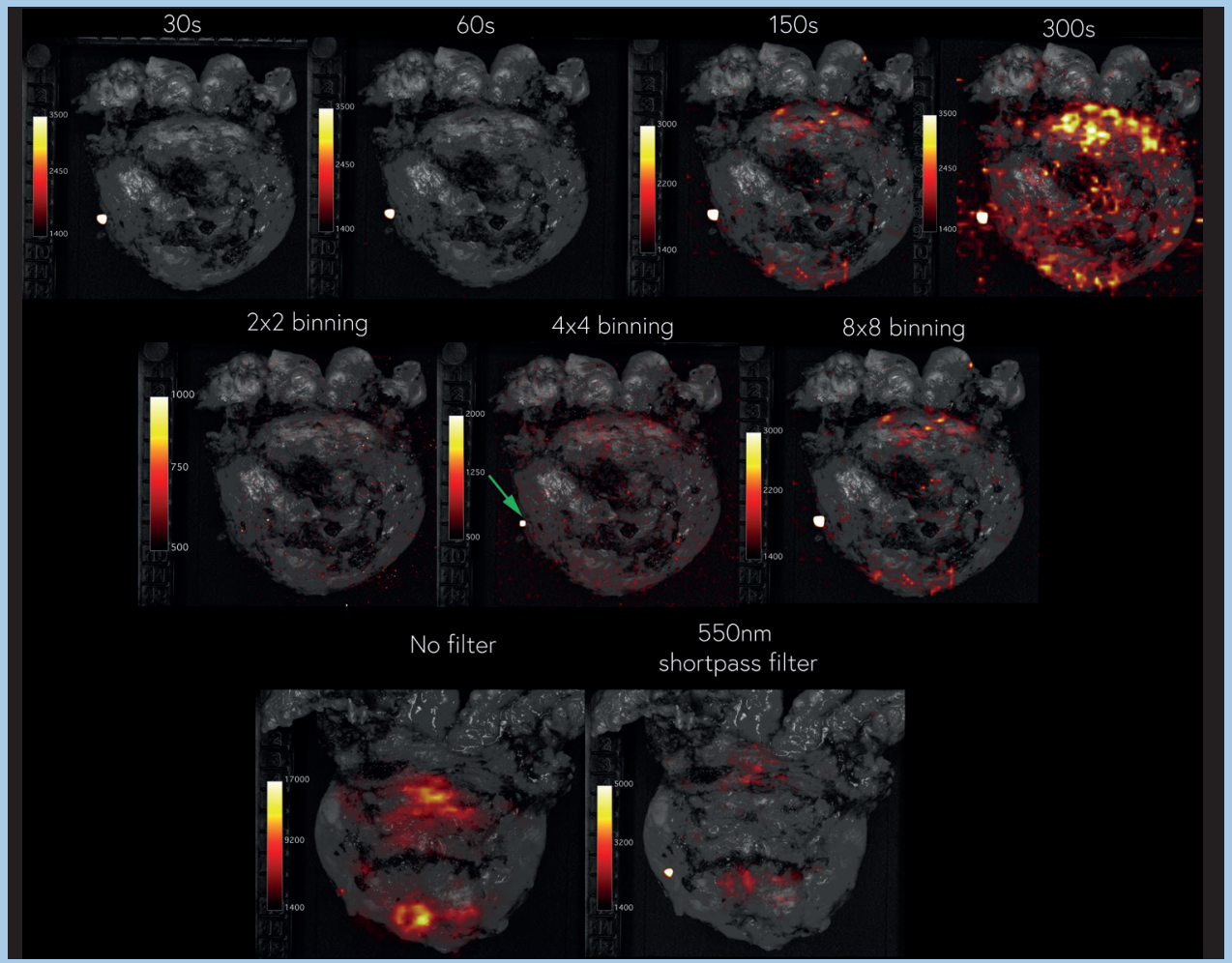

Figure 3. Results of protocol optimisation process with different acquisition settings. The images in the upper row are from patient 1 who had a histopathological PSM at the apex. All images were acquired with $8 \times 8$ pixel binning and $550 \mathrm{~nm}$ shortpass filter but with different exposure times of 30s [TBR:1.10], 60s (TBR: 1.18), 150s (TBR: 1.85] and 300s (TBR: 1.98). The images in the centre row are also from patient 1and were acquired with 150s exposure time and filter, but different pixel binning $2 \times 2$ (TBR: 1.06), $4 \times 4$ [TBR: 1.26] and $8 \times 8$ (TBR: 1.85]. The bottom row images are from patient 3 and were acquired with $150 \mathrm{~s}$ exposure time and $8 \times 8$ binning, but without a filter (TBR: 4.33 ] and with filter (TBR: 1.85 ), respectively. Note that the area of increased signal with the green arrow appears in all non-background subtracted images; this is an artefact from a defect pixel in the camera of the CLI system, and can be ignored. The colour bar indicates the scale of counts. 


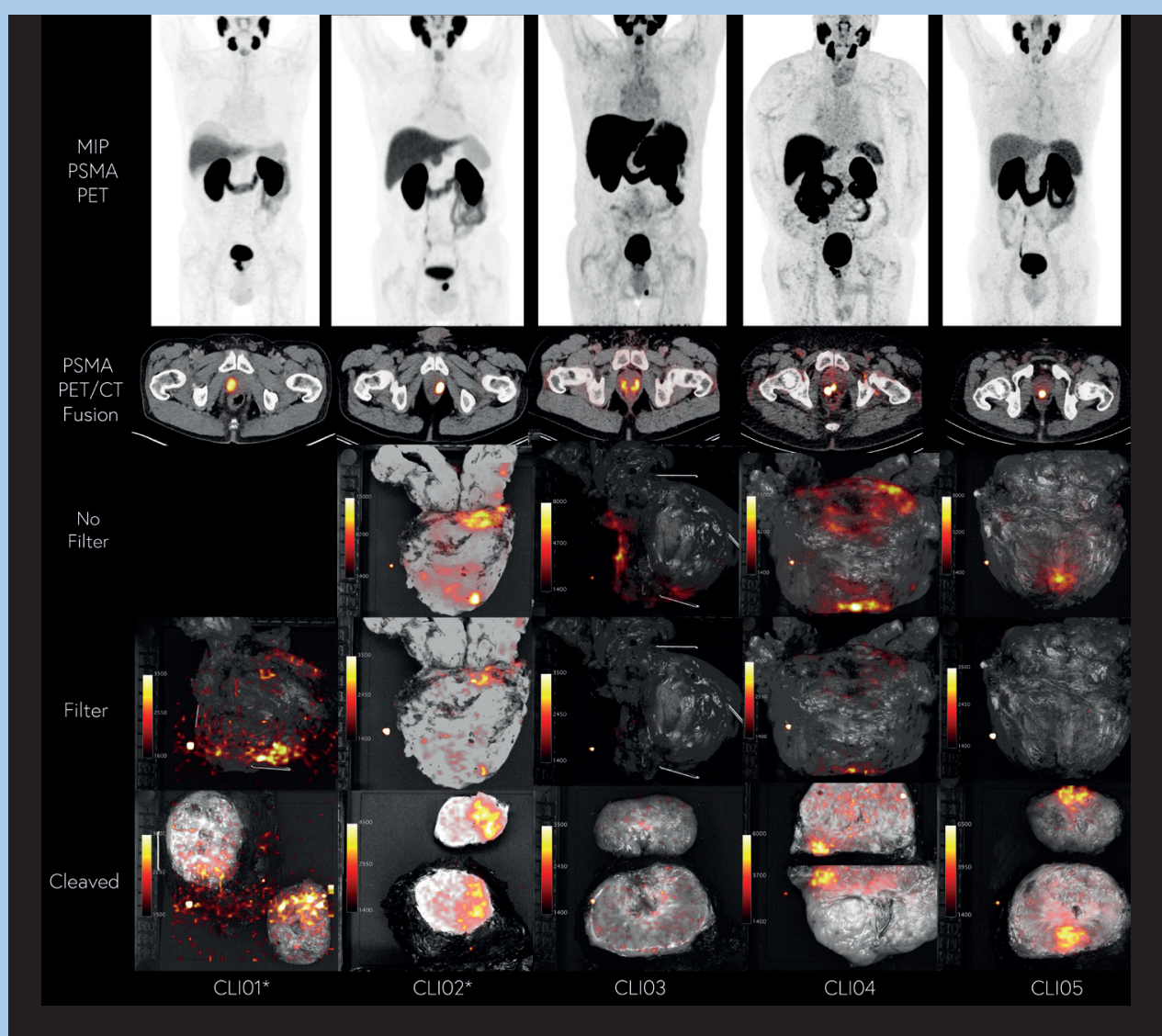

Figure 4. An overview of the PET/CT scans and CLI images of all five patients. The MIP of the PSMA PET-scan. Note that these scans were obtained using ${ }^{68} \mathrm{Ga}$-HBEDCC-PSMA [patient 5] and ${ }^{18} \mathrm{~F}-\mathrm{DCF} P \mathrm{FL}$ [patient 1, 2, 3, 4), hence the difference in PSMA-tracer distribution. The transverse image of the fused PSMA PET/CT scan at the level of the prostate lesion. Non-filtered CLI images of intact prostate specimen. Note that patient one did not have a non-filtered image taken. Filtered CLI images of intact prostate specimen. Non-filtered CLI images of the cleaved prostate. The prostate specimen was cleaved at $-1 \mathrm{~cm}$ from the apex. Note that in patient 3 was not located in the apex region but in the base and therefore no tumour signal is visible on the CLI image. Scaling of the PET-scans was performed based on the intensity of the liver; CLI images were scaled visually based on the benign background signal. The displayed CLI images are the intraoperative images that are not corrected for background which explains the presence of the defective pixel. All CLI images were acquired with 150 s exposure time and 8 x8 binning. *The patient numbers marked with a star had a PSM based on histopathology. 


\section{Discussion}

In the current study, we present the first-in-man usage of intraoperative ${ }^{68} \mathrm{Ga}-\mathrm{PS}$ MA-11 CLI in primary PCa. The results from the first patients enabled optimisation of the imaging protocol, and demonstrated the technical ability to correctly identify PSMs. The use of optical filters improves the ability to visually distinguish between PSM and NSM on CLI, a finding that has not been reported before. Importantly, the performed workflow was considered clinically feasible and safe for surgical staff in terms of radiation exposure levels.

These initial results show that by using an administered activity of $65 \mathrm{MBq}{ }^{68} \mathrm{Ga}-\mathrm{PS}$ MA-11, a 150s acquisition time with $8 \times 8$ pixel binning, and specimen imaging with and without $550 \mathrm{~nm}$ shortpass filter, a PSM can be successfully detected. Compared with the published ${ }^{18} \mathrm{~F}-\mathrm{FDG} \mathrm{CLI}$ results in breast cancer, the current protocol uses a 3 times lower activity and a 2 times shorter acquisition time which is possible due to the higher tracer uptake and Cerenkov yield of ${ }^{68} \mathrm{Ga}$-PSMA-11 [18]. Despite the longer duration of prostate cancer surgery, the lower injected activity and shorter half-life resulted in a roughly 2 times lower staff exposure. The radiation exposure to personnel, a maximum of $0.016 \mathrm{mS} v$ per procedure, were within the International Commission on Radiological Protection limits [25]. Based on these values a single scrub nurse can perform a minimum of $62 \mathrm{CLI}$ procedures before exceeding the limits; a surgeon can perform $200 \mathrm{CLI}$ procedures. All 6 sides of the prostate could be assessed with the CLI within approximately 20 minutes, which is well within the time window acceptable for intraoperative use. Although CLI may still delay surgery to some extent, the procedure is approximately twice as fast as a typical NeuroSAFE procedure that is only performed on the posterolateral surface of the prostate. Furthermore, CLI enables whole-specimen assessment in the operating theatre without the need for a dedicated Pathology department, contrarily to NeurOSAFE. Next, when a lymph node dissection is performed as well, the surgery can continue with the dissection while $\mathrm{CLI}$ imaging of the prostate occurs.

In 3 out of 5 patients the histopathological PSMs and NSMs were correctly detected on CLI. In two patients a hotspot on the Cerenkov image from the intact prostate was obtained from a tumour deposit at $\leq 0.1 \mathrm{~mm}$ from the inked surface. These false-positive results can be explained by the physical properties of ${ }^{8} \mathrm{Ga}$. The CLI signal is not produced in one spatial location, but instead along the positron trajectory in tissue $\left( \pm 2.8 \mathrm{~mm}\right.$ on average for ${ }^{68} \mathrm{Ga}$, which is the same as the PET range) 
[26]. Although the use of an optical filter restricts the detected signal to enable visualisation of activity on the surface [27], it remains hard to quantify from what depth this activity actually originates. As identified in this first-in-man study, depth estimation is a key challenge of CLI and an important limitation compared with NeurOSAFE. The spatial accuracy that is achieved with histopathological evaluation is far greater than will ever be achieved using CLI. This aspect is further accomplished by the fact that it is difficult to estimate the real amount of activity present in the prostate after administration based on the Cerenkov images alone. In the future, the amount of activity present in the excised prostate specimen, could be measured using dose calibrators.

A quantitative approach could help to discriminate between benign and tumour based on a threshold value, rather than on visual interpretation alone, since visual assessment is inherently prone to subjective window-level settings, and may result in erroneous identification of hotspots. In this study, each prostate was cleaved to enable visualisation and quantification of benign and cancerous tissue in the apex. This resulted in a mean $T B R \geq 2$, showing that the technology is able to sufficiently differentiate between tumour and benign tissue. Still, the process of cleaving is not preferred in the eventual work up, as it is time intensive and requires additional training. Our ongoing study will explore the possibility to define a TBR based on preoperative PET/CT scans to recognize and to quantify potential areas that have a higher risk for PSMs on Cerenkov images based on radiance values. However, in the current feasibility study the numbers were too small to make quantitative conclusions from the radiance levels. For quantification the time between injection and imaging, administered activity, can be used to normalise the data. Additional variables that influence the CLI signal that are more challenging to account for are the PSMA expression of the tumour, and the fact that CLI uses optical imaging which means that scattering and attenuation from superficial tissue and blood can alter the Cerenkov signal intensity.

Though the current histopathological definition of a PSM is $0 \mathrm{~mm}$ (i.e., tumour on ink) [24], it is suggested that CLI could still provide valuable information on margin-status, not only to the surgeon but also to inform the pathologist about areas at risk to guide more detailed histopathological evaluation. Another possibility is to guide the NeuroSAFE procedure itself by indicating suspicious areas, so that only those areas could be sampled for NeuroSAFE assessment, thereby reducing the risk of sampling errors and the duration of the NeuroSAFE procedure. 
The current feasibility study has several limitations. Firstly, this feasibility study only addresses a small selective population. The patients were selected based on a high chance of a PSM, thus not representative for the general population that will undergo RARP. Still, the included patients are the ones that could benefit the most from intraoperative margin assessment [28]. Secondly, the administered activity varied between the patients. The ${ }^{68} \mathrm{Ga}$-PSMA-11 was produced locally at specific time slots each day due to the fact that this is a generator product. Thus, the actual time of surgery needs to correspond to the ${ }^{68} \mathrm{Ga}-\mathrm{PSMA}-11$ production time. Batches for multiple patients are retrieved from one production, therefore once the syringe is prepared for the study patient, and surgery is delayed, the activity cannot be adjusted accordingly. This is further complicated by the relatively short half-life of ${ }^{68} \mathrm{Ga}$ (68 minutes). To account for lower ${ }^{68} \mathrm{Ga}-\mathrm{PSMA}-11$ activity levels due to delays in the start of surgery, the exposure time could be increased, thereby increasing TBR and ultimately aiding the visual identification of PSM on CLI. Still, variation in activity present in the prostate is normal considering a different PSMA expression between patients, and even within one prostatic lesion. This could be correlated to the SUV from preoperative PET/CT scans. 


\section{Conclusion}

This first in man study demonstrates that ${ }^{68} \mathrm{Ga}-\mathrm{PSMA}-11 \mathrm{CLI}$ is a promising and safe technique for intraoperative margin assessment in PCa. The validated acquisition settings enable detection of PSMs within an acceptable time window for intraoperative use, and acceptable radiation exposure to staff. The ongoing trial will further evaluate the diagnostic accuracy of CLI in a larger population and will assess the ability to establish a quantitative threshold to discriminate PSM from NSM, in addition to visual inspection alone. 


\section{REFERENCES}

1. Yossepowitch O, Briganti A, Eastham JA, et al. Positive Surgical Margins After Radical Prostatectomy: A Systematic Review and Contemporary Update. Eur Urol. 2014;65:303-313.

2. Stephenson AJ, Wood DP, Kattan MW, et al. Location, Extent and Number of Positive Surgical Margins Do Not Improve Accuracy of Predicting Prostate Cancer Recurrence After Radical Prostatectomy. J Urol. 2009;182:1357-1363.

3. Silberstein J, Eastham J. Significance and management of positive surgical margins at the time of radical prostatectomy. Indian J Urol. 2014;30:423-428.

4. Bolla M, van Poppel H, Tombal B, et al. Postoperative radiotherapy after radical prostatectomy for high-risk prostate cancer: long-term results of a randomised controlled trial (EORTC trial 22911). Lancet. 2012;380:2018-2027.

5. Schlomm T, Tennstedt P, Huxhold C, et al. Neurovascular structure-adjacent frozen-section examination (NeuroSAFE) increases nerve-sparing frequency and reduces positive surgical margins in open and robot-assisted laparoscopic radical prostatectomy: experience after 11,069 consecutive patients. Eur Urol. 2012;62:333-340.

6. Mirmilstein G, Rai BP, Gbolahan $\mathrm{O}$, et al. The neurovascular structure-adjacent frozen-section examination (NeuroSAFE) approach to nerve sparing in robot-assisted laparoscopic radical prostatectomy in a British setting - a prospective observational comparative study. BJU Int. 2018;121:854-862.

7. Beyer B, Schlomm T, Tennstedt P, et al. A Feasible and Time-efficient Adaptation of NeuroSAFE for da Vinci Robot-assisted Radical Prostatectomy. Eur Urol. 2014;66:138-144.

8. Dinneen EP, Van Der Slot M, Adasonla K, et al. Intraoperative Frozen Section for Margin Evaluation During Radical Prostatectomy: A Systematic Review. Eur Urol Focus. 2019;6:664-673

9. Spinelli $A E$, D'Ambrosio $D$, Calderan $L$, et al. Cerenkov radiation allows in vivo optical imaging of positron emitting radiotracers. Phys Med Biol. 2010;55:483-895.

10. Robertson R, Germanos MS, Li C, et al. Optical imaging of Cerenkov light generation from positron-emitting radiotracers. Phys Med Biol. 2009;54:355-365.

11. Ruggiero A, Holland JP, Lewis JS, et al. Cerenkov Luminescence Imaging of Medical Isotopes. J Nucl Med. 2010;51:1123-1130.

12. Ciarrocchi E, Belcari N. Cerenkov luminescence imaging: physics principles and potential applications in biomedical sciences. EJNMMI Phys. 2017;4:14.

13. Beattie BJ, Thorek DLJ, Schmidtlein CR, et al. Quantitative Modeling of Cerenkov Light Production Efficiency from Medical Radionuclides. PLoS One. 2012;7:e31402.

14. Holland JP, Normand G, Ruggiero A, et al. Intraoperative imaging of positron emission tomographic radiotracers using Cerenkov luminescence emissions. Mol Imaging. 2011;10:177-186.

15. Thorek DLJ, Ogirala A, Beattie BJ, et al. Quantitative imaging of disease signatures through radioactive decay signal conversion. Nat Med. 2013;19:1345-1350.

16. Gill RK, Mitchell GS, Cherry SR. Computed Cerenkov luminescence yields for radionuclides used in biology and medicine. Phys Med Biol. 2015;60:4263-4280

17. Spinelli $A E$, Ferdeghini M, Cavedon C, et al. First human Cerenkography. J Biomed Opt. 2013;18:020502.

18. Grootendorst MR, Cariati M, Pinder SE, et al. Intraoperative Assessment of Tumor Resection Margins in Breast-Conserving Surgery Using ${ }^{18}$ F-FDG Cerenkov Luminescence Imaging: A Firstin-Human Feasibility Study. J Nucl Med. 2017;58:891-898. 
19. Liu Y. Diagnostic role of fluorodeoxyglucose positron emission tomography-computed tomography in prostate cancer. Oncol Lett. 2014;7:2013-2018.

20. Jadvar H. Is There Use for FDG-PET in Prostate Cancer? Semin Nucl Med. 2016;46:502-506.

21. Corfield J, Perera M, Bolton D, et al. ${ }^{68} \mathrm{Ga}$-prostate specific membrane antigen (PSMA) positron emission tomography (PET) for primary staging of high-risk prostate cancer: a systematic review. World J Urol. 2018;36:519-27.

22. olde Heuvel J, de Wit-van der Veen BJ, Vyas KN, et al. Performance evaluation of Cerenkov luminescence imaging: a comparison of ${ }^{68} \mathrm{Ga}$ with ${ }^{18} \mathrm{~F}$. EJNMMI Phys. 2019;6:17.

23. Ciarrocchi E, Vanhove C, Descamps B, et al. Performance evaluation of the LightPath imaging system for intraoperative Cerenkov luminescence imaging. Phys Medica. 2018;52:122-128.

24. Tan PH, Cheng L, Srigley JR, et al. International Society of Urological Pathology (ISUP) consensus conference on handling and staging of radical prostatectomy specimens. Working group 5: surgical margins. Mod Pathol. 2011;24:48-57.

25. The 2007 Recommendations of the International Commission on Radiological Protection. ICRP publication 103. Ann ICRP. 2007;37:1-332.

26. Moses WW. Fundamental limits of spatial resolution in PET. Nucl Instruments Methods Phys. Res. A. 2011;648: S236-240.

27. Sandell J, Zhu T. A review of in-vivo optical properties of human tissues and its impact on PDT. J Biophotonics. 2013;80:631-637.

28. Wieder JA, Soloway MS. Incidence, etiology, location, prevention and treatment of positive surgical margins after radical prostatectomy for prostate cancer. J Urol. 1998;160:299-315.

29. Buyyounouski MK, Choyke PL, McKenney JK, et al. Prostate cancer - major changes in the American Joint Committee on Cancer eighth edition cancer staging manual. CA. Cancer J Clin. 2017;67:245-53. 



\section{CHAPTER 7}

\section{CERENKOV LUMINESCENCE IMAGING IN PROSTATE CANCER:}

NOT THE ONLY LIGHT THAT SHINES

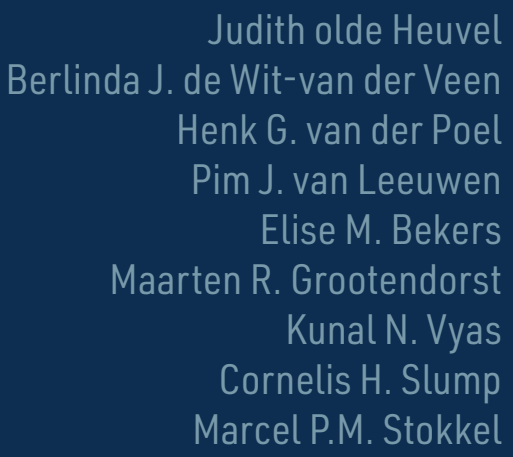

Resubmitted after major revisions to J Nucl Med. 


\section{Abstract}

\section{INTRODUCTION}

Cerenkov luminescence imaging $(\mathrm{CLI})$ is a novel imaging technology that might have the ability to assess surgical margins intraoperatively at radical prostatectomy using gallium-68 prostate-specific membrane antigen ( ${ }^{68} \mathrm{Ga}-\mathrm{PSMA}-11$ ). This study evaluates the accuracy of $\mathrm{CLI}$ compared to histopathology and as secondary objective investigates the characteristics of a newly identified bioluminescence signal.

\section{Methods}

After intravenous injection of $\sim 100 \mathrm{MBq}{ }^{68} \mathrm{Ga}-\mathrm{PSMA}-11$ intraoperatively, all excised specimens were imaged with CLI. Areas of increased signal were marked for histopathological comparison and scored for likelihood of being a positive surgical margin (PSM) using a 5-point Likert scale. In addition, the bioluminescence signal was investigated in three radioactive and three non-radioactive specimens using CLI.

\section{RESULtS}

In 15 patients, the agreement between CLI and histopathology was 59\%; this improved to $82 \%$ when including close surgical margins ( $\leq 1 \mathrm{~mm}$ ). In six hotspots, CLI correctly identified PSMs on histopathology, located at the apex and mid-prostate. In all 15 patients an increased signal at the prostate base was observed, without the presence of the primary tumour in this area in eight patients. This bioluminescence signal was also observed in non-radioactive prostate specimens, with a halflife of $48 \pm 11 \mathrm{~min}$. The bioluminescence hampered the visual interpretation of four PSMs at the base.

\section{CONCLUSION}

$\mathrm{CLI}$ is able to correctly identify the margin status, including close margins, in $82 \%$ of the cases. The presence of a diathermy-induced bioluminescent signal hampered image interpretation, especially at the base of the prostate. In the current form, CLI is most applicable to detect PSMs and close margins in the apex and mid-prostate. 


\section{Introduction}

Positive surgical margins (PSMs) on histopathology occur in 11-38\% of the patients undergoing a radical prostatectomy $[1,2]$. These men have a higher risk of receiving with adjuvant local radical therapy [1,3]. Multiple intraoperative margin assessment technologies are developed that aim to enable a radical excision(4). Of these, NeurOSAFE is currently the only method used for clinical decision making with good diagnostic accuracy (sensitivity: 93.5\% \& specificity: 98.8\%) [5], but the procedure is labour intensive, time consuming and costly and prone to sampling errors and therefore only used in a small number of centres [6]. In addition, a study showed that $70 \%$ of the secondary resections due to a PSM on NeuroSAFE did not contain tumour [7].

A recently introduced technology to aid intraoperative margin assessment is Cerenkov luminescence imaging (CLI) [8-10]. Cerenkov radiation is induced when a charged particle travels faster than the velocity of light through tissue, thus polarizing the tissue. When the locally polarized medium returns to its ground state, Cerenkov photons are emitted [10]. Tumour-specific tracers, like gallium-68 prostate-specific-membrane antigen $\left({ }^{68} \mathrm{Ga}-\mathrm{PSMA}-11\right)$, induce Cerenkov radiation and the detected rays from superficial layers can guide towards areas with high suspicion of a PSM. Recent studies on this application have already shown the feasibility and safety of this technique $[11,12]$.

During a radical prostatectomy, electro surgery (i.e. diathermy) is used for cutting and coagulation of tissue. This technique uses radio-frequency currents to increase the intracellular temperature, which in turn can result in vaporization and blood clotting. A preclinical study suggested that heat may induce bioluminescence with wavelengths between 400-710 nm [13,14], which overlap with the Cerenkov spectrum (400-1000 nm) [15]. Thus, this bioluminescence might interfere with the CLI signal, indicating a potential limitation $[13,14]$. However, these studies were conducted with animal tissue and used other thermal sources, thus the spectrum, halflife and other features in human perfused tissue after diathermy application are unknown.

In our prior study we have investigated the technical feasibility of ${ }^{68} \mathrm{Ga}$-PSMA-11 $\mathrm{CLI}$ for intraoperative margin assessment in a small male population with prostate 
cancer (PCa) [12]. This consecutive study evaluates the accuracy of CLI compared to histopathology in a larger population, and as a secondary objective investigates the characteristics of a novel bioluminescent signal observed on CLI. 


\section{Methods}

The present study was approved by the local ethics committee (NL8256, trialregister.nl). Patients with high-risk primary PCa scheduled for robot-assisted radical prostatectomy were enrolled in this trial after written informed consent was obtained. The inclusion criteria were patients with biopsy proven prostate cancer with a tumour lesion $\geq 1.5 \mathrm{~cm}$ on MRI and a PSMA-positive intra-prostatic lesion on PSMA $\mathrm{PET} / \mathrm{CT}$. The exclusion criterion was the use of Indocyanine green during surgery, since this may influence the CLI measurements. In all patients, CLI images were not used for surgical or histopathological decision making.

\section{SURGERY AND INTRAOPERATIVE CLI}

An intravenous injection of $\sim 100 \mathrm{MBq}{ }^{68} \mathrm{Ga}-\mathrm{Glu}-$ urea-Lys (Ahx)-HBED-CC (ABX $\mathrm{GmbH}$, Radeberg, Germany) was given after the da Vinci ${ }^{\circledast}$ surgical system was docked. Immediately after prostatectomy, ex vivo Cerenkov images of the prostate specimen were acquired with the LightPath ${ }^{\circledast}$ system (Lightpoint Medical Ltd, Chesham, UK) using the following imaging parameters: exposure time of 150s, $8 \times 8$ pixel binning (pixel resolution $938 \mu \mathrm{m}$ ), no optical filter. A $550 \mathrm{~nm}$ shortpass filter was applied when areas of increased signal (called "hotspots") were visualized on the non-filtered images.

\section{UNDERSTANDING THE CHARACTERISTICS OF THE BIOLUMINESCENT SIGNAL}

\section{Effect of urine contamination on CLI}

Since ${ }^{68} \mathrm{Ga}-\mathrm{PSMA}-11$ undergoes renal clearance, it was verified if radioactive urine might affect CLI, by first imaging three specimens without rinsing from potential blood and urine contamination. Next, it was rinsed twice with $500 \mathrm{~mL} 0.9 \%$ sodium chloride $(\mathrm{NaCl})$ prior to subsequent imaging. The visual and quantitative difference between three images in the rinsing process were compared. In the other patients, $\mathrm{CLI}$ was performed after rinsing the prostate once with $500 \mathrm{~mL}$ of $0.9 \% \mathrm{NaCl}$.

\section{$\mathrm{CLI}$ and flexible autoradiography on non-radioactive prostate specimens}

The prostate specimens of three patients undergoing a prostatectomy without injection of ${ }^{68} \mathrm{Ga}-\mathrm{PSMA}-11$ (the 'non-radioactive specimens') were imaged with CLI to investigate the characteristics of the bioluminescence that was observed on the radioactive specimens. On the images multiple regions of interest were drawn to quantify any bioluminescence signal and the reduction in this signal by applying a $550 \mathrm{~nm}$ shortpass filter. The half-life of bioluminescence was determined by consec- 
utive imaging over 60 minutes.

To investigate an alternative method that is potentially less susceptible to bioluminescence than CLI, flexible autoradiography (FAR) was tested on non-radioactive specimens. A $12 \mu \mathrm{m}$ flexible scintillating film (Lightpoint Medical Ltd. and Scintacor Ltd., UK) was draped over the specimen and imaged in LightPath using the same imaging parameters as for CLI. The opaque nature of the scintillator may block some of the bioluminescent photons while detecting the positrons emitted by ${ }^{68} \mathrm{Ga}-\mathrm{PS}$ MA-11 [16]. The reduction in bioluminescent signal from photon absorption by the FAR was assessed, as well as the half-life over a 60 minute measurement period.

\section{FAR on ${ }^{68} \mathrm{Ga}$-PSMA-1I containing prostate specimens}

In three CLI patients, FAR was used on ${ }^{68} \mathrm{Ga}$-PSMA-11 containing prostate specimens in addition to CLI imaging. Images with and without $550 \mathrm{~nm}$ shortpass filter were acquired to investigate the effect of the FAR on the signal originating from ${ }^{68} \mathrm{Ga}-\mathrm{PSMA}-11$ uptake and in relation to bioluminescence.

\section{HISTOPATHOLOGY}

After CLI acquisitions, the specimens were send for standard histopathological examination by an experienced uropathologist (EMB). A PSM was defined as cancer extending into the inked surface in accordance with the guidelines [17]. At the marked locations the tumour to margin distance was measured.

\section{IMAGE ANALYSIS}

Image quantification and pre-processing was performed in MATLAB R2017b (The MathWorks, Natick, USA) as previously described [12]. The mean radiance (photons $/ \mathrm{s} / \mathrm{cm}^{2} /$ steradian) was measured in areas showing 'hotspots' (suspected tumour) and in areas with no increased signal (tissue background), the tumour-to-tissue background ratios (TBRs) were calculated. Hotspots were defined as signals higher than 3 times the standard deviation (sd) of the empty background[18]. Visual interpretation of all images was performed postoperatively by two independent readers (JoH, BJdWvdV). All hotspots were scored to assess the likelihood of the spot being a PSM using a 5-point Likert scale (1-likely negative surgical margin (NSM), 5-likely PSM). Features used to score hotspots were: knowledge of the location of the tumour on PSMA PET/CT, size of the hotspot (required size $\geq 1 \mathrm{~mm}$ due to $8 \times 8$ binning), intensity above the tissue background, and usage of diathermy. Observers were blinded for each other's scores, any disagreement was settled in 
consensus. A score on the Likert scale (LS) of 4 and 5 was considered PSM in further analysis, 1-3 as NSM. The extent of the PSMs was measured on CLI images (longest axis) and compared to the extent measured on histopathology. Agreement between the margin status on histopathology and CLI was assessed in three areas of the prostate: base (top $1 \mathrm{~cm}$ ), mid-gland and apex (bottom $1 \mathrm{~cm})$. The agreement was calculated with and without additional image interpretation (using the LS), and with and without inclusion of a close surgical margin (CSM $\leq 1 \mathrm{~mm}$ tumour to margin distance). 


\section{Results}

\section{RESULTS INTRAOPERATIVE CLI RADIOACTIVE SPECIMENS}

In this study 17 patients were included. One patient was excluded due to an unsuccessful ${ }^{68} \mathrm{Ga}$-PSMA-11 labelling on the day of the surgery and one due to the use of Indocyanine green, leaving 15 patients to be included in the analysis. The mean injected activity was $69 \pm 27 \mathrm{MBq}$ (range $23-121 \mathrm{MBq}$ ), with on average $70 \pm 15 \mathrm{~min}$ (range 44-105 $\mathrm{min}$ ) between injection and CLI acquisition. The patient and procedure characteristics can be found in Table 1.

Figure 1 displays examples of CLI images showing hotspots identified as PSM, NSM or CSM on histopathology. The overall agreement between CLI (hotspot yes/no) and histopathology with respect to the margin status (PSM or NSM) was 31\% without any image interpretation by the user (Figure 2 \& Supplemental Table 1). This overall agreement improved to $59 \%$ margins when $\leq 1 \mathrm{~mm}$ (CSM) on histopathology were also classified as PSM. Discordance between CLI and histopathology was mainly present at the prostate base.

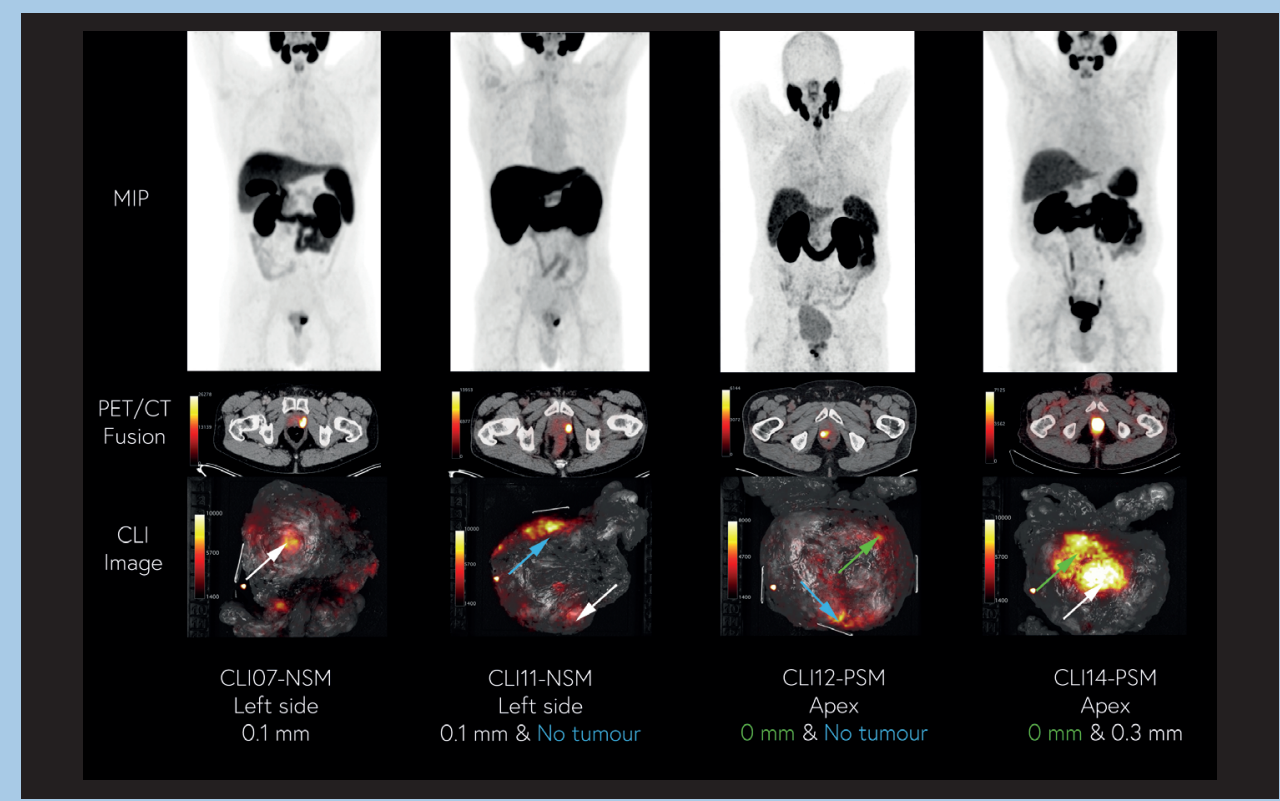

Figure 1. Examples of PET/CT and CLI images from four patients included in this study. Top row: maximum intensity profile [MIP] of the preoperative PSMA PET. Second row: transversal PET/CT slide at the height of the primary tumour. Third row: CLI of excised prostate specimens without an optical filter. The arrows locate the hotspot areas, in green a PSM, in blue an NSM, and in white an NSM with the tumour $\leq 1 \mathrm{~mm}$ from the margin, all defined on histopathology. The corresponding tumour to margin distances on histopathology are noted below the images. 
Please rotate the book for the optimal experience

入

幽.

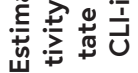

ind

ฐ

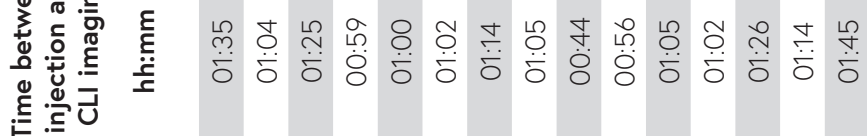

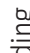

임

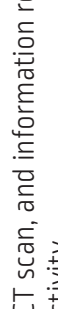

$=\pi$

岩总

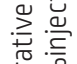

$\frac{0}{\frac{0}{0}} \cdot \frac{0}{\circ}$

응

는

등 䒕

운 흔

$\stackrel{+}{\leftrightarrow}$

沓

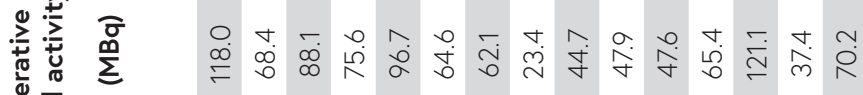

웅

$\stackrel{2}{\stackrel{2}{2}}$

$\dot{\bar{c}}$

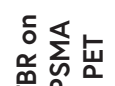

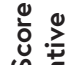

ง

帘

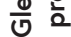

$\vdash . \subseteq$

总竞

苋

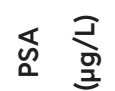

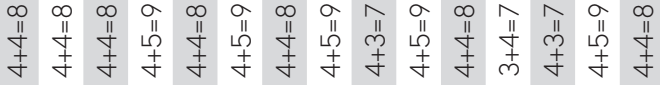

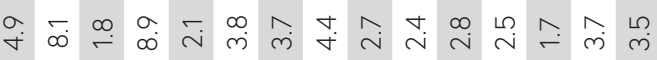

$\frac{\sqrt{6}}{\frac{0}{6}}$

ᄃ

总峲

市

宽

营 응

$\sum_{i}$

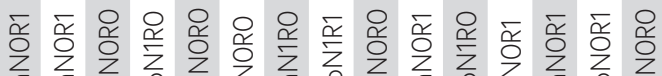

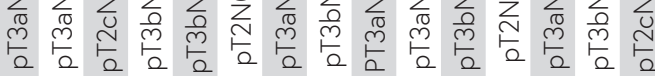

离离

Q

$\frac{1}{\frac{1}{0}}$

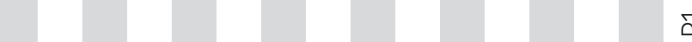

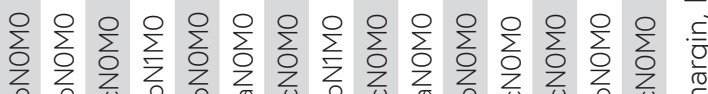

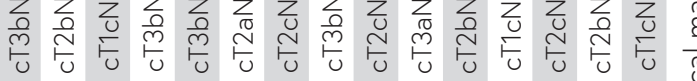

.气ำ

흔 웜

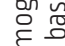

는 원

幽

尊

离竞离

d)

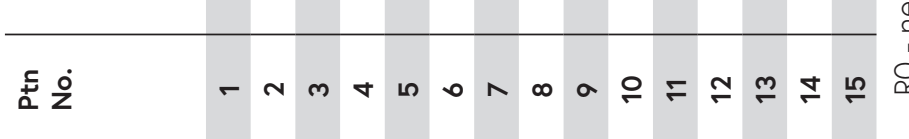


Table 2. Overview of the histopathology and CLI results from the ten histopathological PSMS.

\begin{tabular}{c|cccc}
$\begin{array}{c}\text { Ptn } \\
\text { no. }\end{array}$ & Location of PSM on PA & $\begin{array}{c}\text { Likert score }- \text { TBR } \\
\text { CLI }\end{array}$ & $\begin{array}{c}\text { Gleason score at the } \\
\text { PSM }\end{array}$ & $\begin{array}{c}\text { Extent PSM PA/CLI } \\
\text { (mm) }\end{array}$ \\
\hline $\mathbf{1}$ & Apex right & $5-13.2$ & 8 & $3 / 6.9$ \\
\hline $\mathbf{8}$ & Apex left & $5-5.9$ & 8 & $4 / 4.3$ \\
& Base central & $3-7.1$ & 10 & $14 / 14$ \\
& Base left & $3-6.1$ & 10 & $14 / 12$ \\
\hline 10 & Apex right & $5-8.7$ & 10 & $15 / 12$ \\
\hline $\mathbf{1 2}$ & Base right & $3-6.1$ & 9 & $10 / 9.6$ \\
\hline $\mathbf{1 3}$ & Base central & $3-5.1$ & 8 & $9.0 / 6.7$ \\
\hline $\mathbf{1 4}$ & Apex posterior/ & $5-2.8$ & $3+4=7$ & $6.0 / 6.5$ \\
& mid-prostate & & & $1.0 / 1.4$ \\
\hline & Apex left & $5-2.9$ & 8 & $2.0 / 12.0$ \\
\hline
\end{tabular}

PA - histopathology, PSM - positive surgical margin, TBR - tumour-to-tissue background ratio

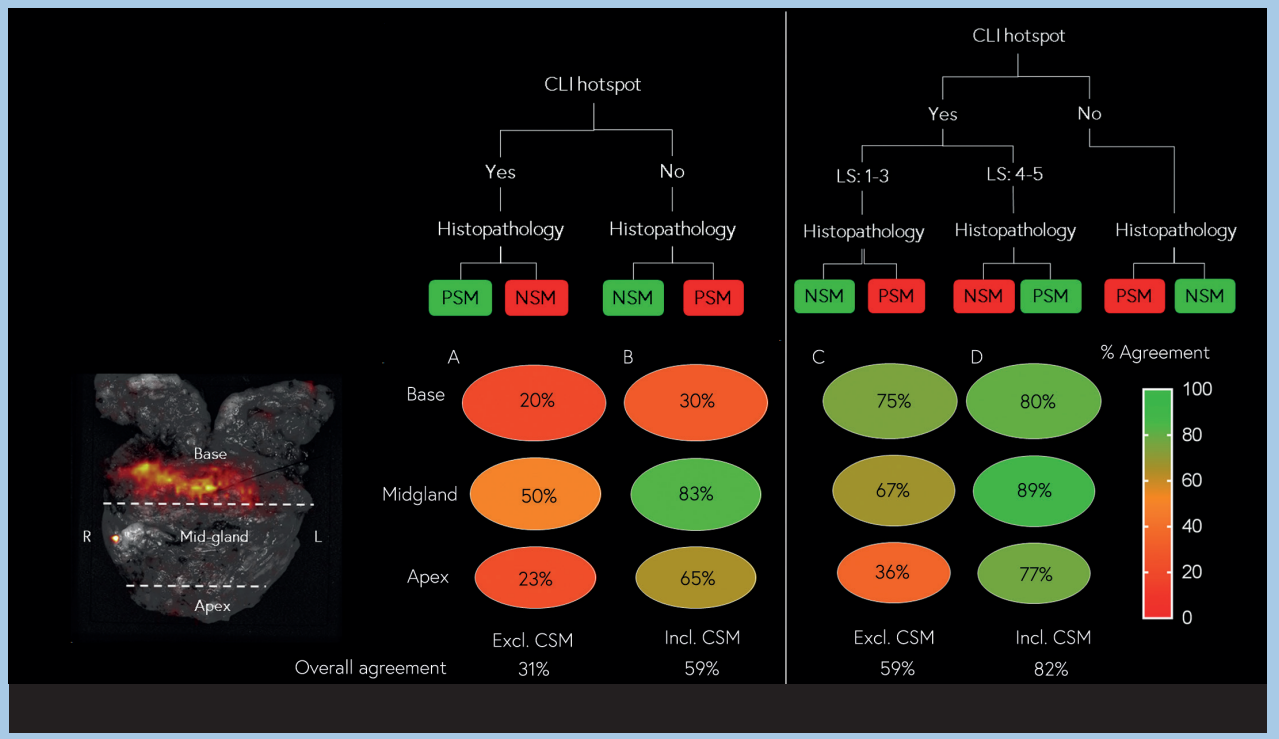

Figure 2. Agreement between CLI and histopathology in all patients divided in 3 regions of the prostate. A] shows the agreement between [LI hotspot [yes/no] and histopathology [PSM/NSM], B] the agreement includes [SM [ $\leq 1 \mathrm{~mm}$ ]. C] agreement when adding a Likert score to the CLI hotspot. D] the agreement with Likert score includes CSM. Overall agreement is noted below the circles.

Additionally, hotspots on CLI were scored using the Likert Scale (LS), taking into account the bioluminescence. A disagreement on the score between readers occurred in eight hotspots, and concordance was settled in consensus. In all these cases, the disagreement concerned 4 versus 5 , or 1 versus 2-3. With the addition this image 
interpretation, the overall agreement between CLI and histopathology was 59\%, which improved to $82 \%$ with inclusion of CSM.

Ten hotspots were actually identified as histopathological confirmed PSM (Table 2); six were detected at the apex/mid-prostate with a $L S \geq 4$ (true positive), and four were detected at the base with a LS of 3 (false negative). There were no proven PSMs that did not show signal on CLI. The extent of PSM measured on CLI and histopathology showed in 5/10 PSMs a difference of less than $1 \mathrm{~mm}$, Table 2.

The average \pm sd TBR of PSM was $7.1 \pm 3.5$, CSM was TBR: $5.0 \pm 1.9$ and for NSM this was $5.8 \pm 3.9$ on a non-filtered image.

In all 15 patients, an intense signal was observed on CLI at the prostate base, Figure 3. In eight of these patients the prostate tumour was not located in the base, as indicated on preoperative PET imaging. These findings indicate the presence of an additional bioluminescence signal, which may explain the high false negative rate at the base.

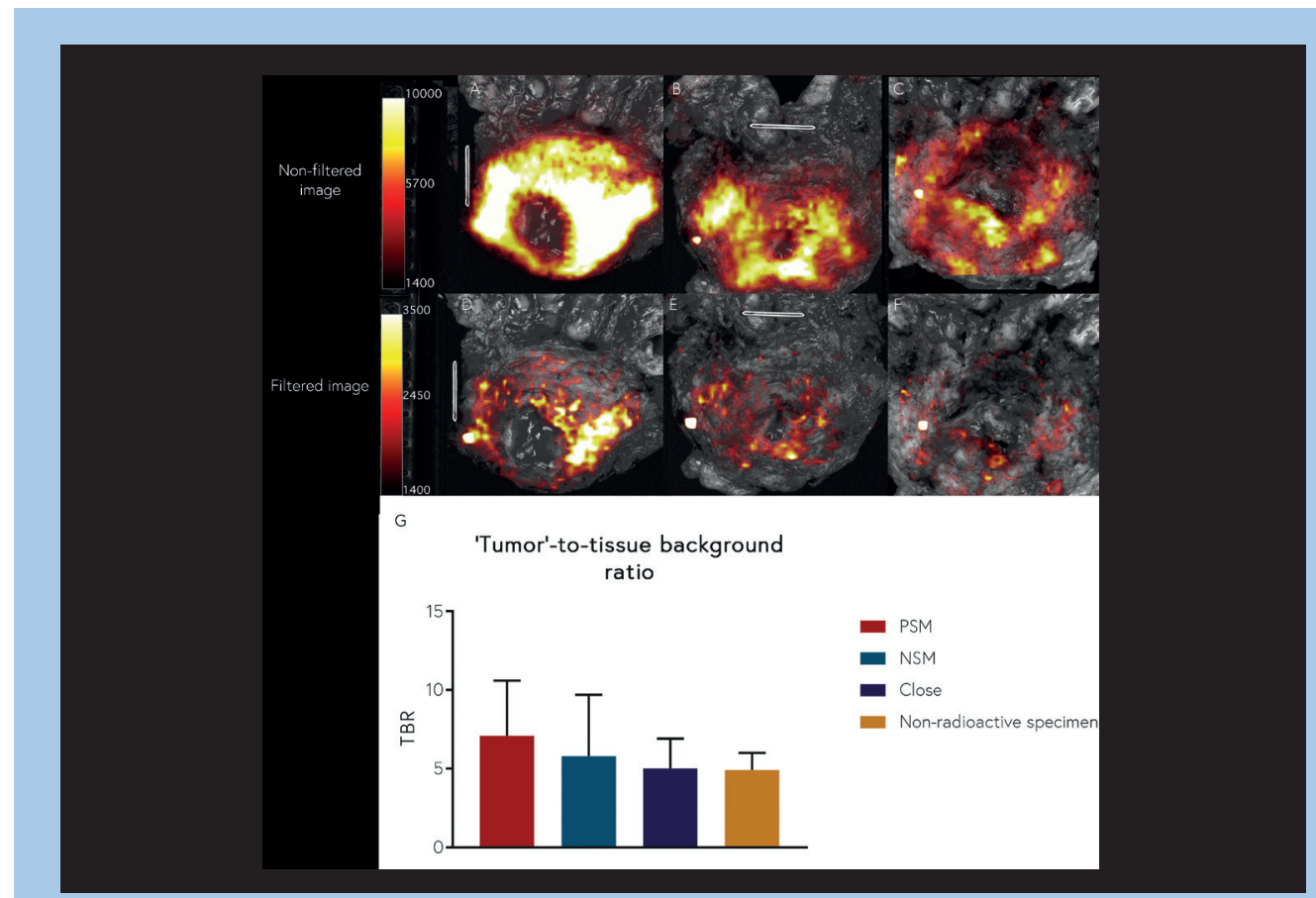

Figure 3. CLI images from the prostate base in ${ }^{68} \mathrm{Ga}-\mathrm{PSMA}-11$ patients and a bar chart showing the TBR of CLI and bioluminescence. Image $A$ and $B$ : unfiltered images of the bioluminescence at the base. Image $D$ and $E$ : the corresponding filtered images. Image $C$ and $F$ : the base of patient 8 , which had multiple PSMs at the base. These images show that visual distinction between of bioluminescence and PSMs is difficult. G: Bar chart displaying the TBR of bioluminescence in non-radioactive specimens and the average TBR of PSM, NSM and CSM in patients. 
Effect of urine contamination on CLI

After rinsing once, the bioluminescence signal at the base was reduced to $93 \%$ of the original CLI signal. After the second rinsing, the signal decreased to $88 \%$ compared to the unrinsed prostate. Time between the rinsed images and unrinsed image was respectively 5 and 10 minutes.

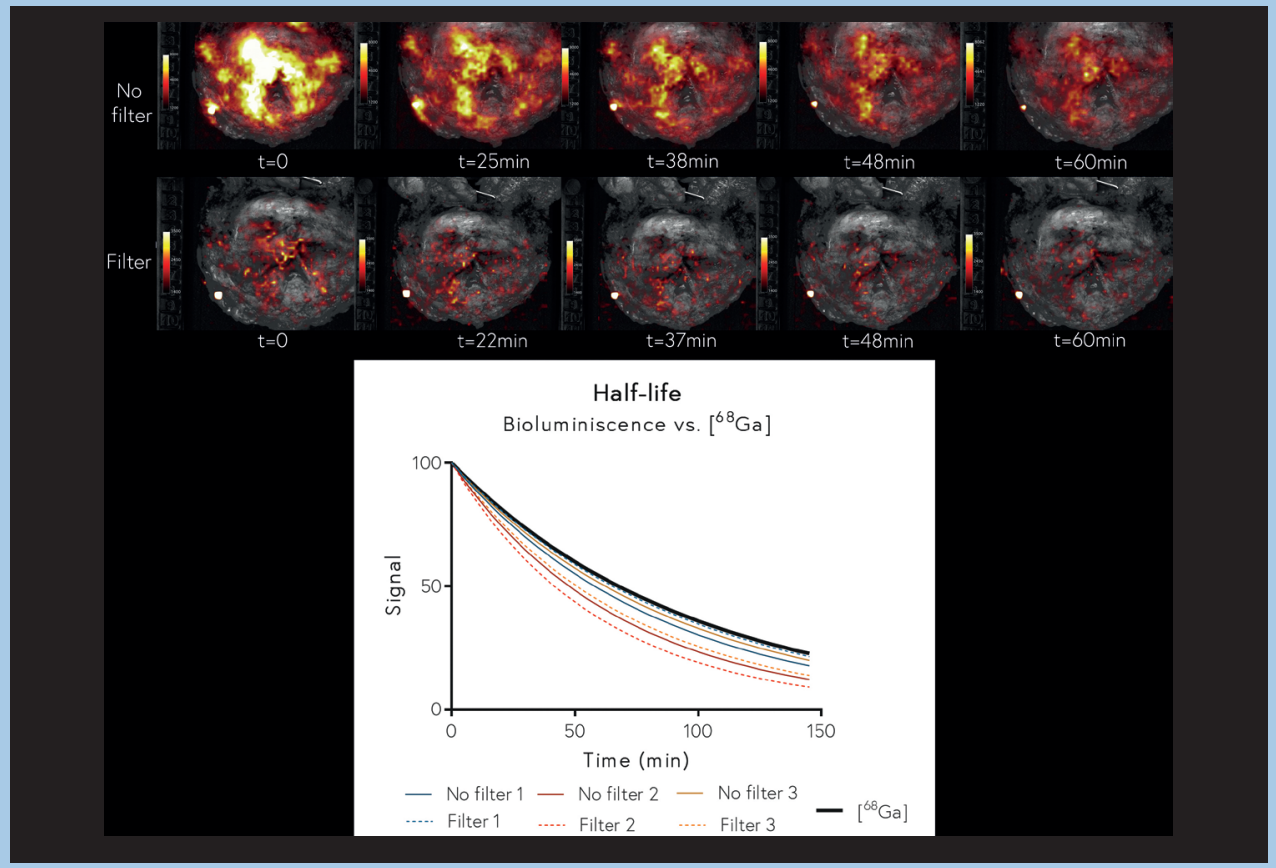

Figure 4. Sequential imaging of non-radioactive prostate specimens to determine the effect of time on the intensity of the bioluminescence signal. Top row: images without filter. Bottom row: images with $550 \mathrm{~nm}$ shortpass filter. Note that the same scaling is used in all images. The graph displays the halflife of bioluminescence in three non-radioactive specimens on filtered and non-filtered images, and the half-life of ${ }^{68} \mathrm{Ga}$.

\section{CLI and FAR on non-radioactive prostate specimens}

The bioluminescent signal was also visible on CLI images from three 'non-radioactive specimens', primarily at the base, Figure 4. This observation underlines the hypothesis that bioluminescence is not originating from ${ }^{68} \mathrm{Ga}$-PSMA-11. The TBR of the bioluminescence was $4.9 \pm 1.1$ on the unfiltered image. The half-life without an optical filter was $48 \pm 11 \mathrm{~min}$, and $52 \pm 16 \mathrm{~min}$ with the $550 \mathrm{~nm}$ filter. The application of a $550 \mathrm{~nm}$ shortpass filter resulted in a reduction to $15 \%$ of the original bioluminescence signal, which was comparable to the $\mathrm{CLI}$ signal reduction by filtering in radioactive specimens (18\%). The application of FAR resulted in the reduction of the bioluminescent signal to $60 \%$ of the original CLI image, both with and without $550 \mathrm{~nm}$ filtering, Figure 5 . 


\section{FAR on ${ }^{68} \mathrm{Ga}$-PSMA-II prostate specimens}

FAR put on the radioactive specimens reduced the bioluminescence to $70 \%$ at locations where diathermy was applied. Whereas tumour and tissue background signals were amplified by FAR on average 3.2 times the original signal (Figure 5 and Supplemental Figure 1).

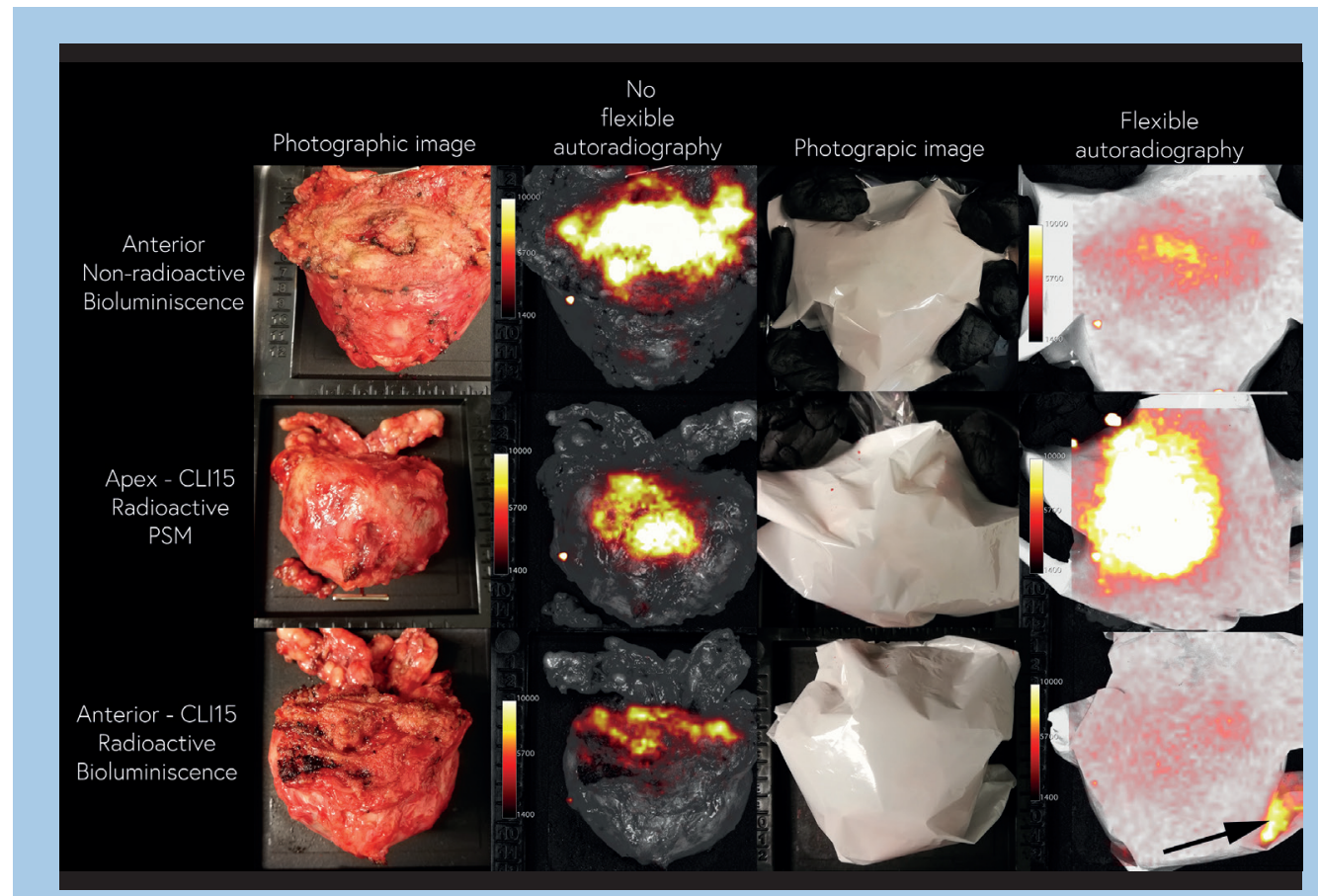

Figure 5. FAR images of ${ }^{68} \mathrm{Ga}-\mathrm{PSMA}-11$ containing prostate specimens and non-radioactive prostate specimens to investigate the effect of FAR on the tumour and bioluminescent signal levels. FAR reduced the bioluminescence in both the non-radioactive [60\%] and radioactive specimen [70\%] [top and bottom row]. FAR amplified the signal originating from the tumour [centre row]. The black arrow shows contamination of the scintillator. Note that the same scaling is used for all images. 


\section{Discussion}

The primary aim of the current study was to assess the concordance in margin status between intraoperative CLI and postoperative histopathology. This study showed that the agreement between CLI and histopathology ranged from 31-82\% depending on definition of PSM and application of image interpretation using the Likert score. The secondary aim of the study was to characterize the observed bioluminescence identified on CLI. It was concluded that this signal is unrelated to ${ }^{68} \mathrm{Ga}-\mathrm{PSMA}-11$, has a mean signal-to-background ratio of $4.9 \pm 1.1$ and a half-life of $48 \pm 11$ minutes. This bioluminescence proved relevant as it hampered easy identification of 4/10 PSMs on CLI, all four located at the base.

When considering the histopathological PSM definition as tumour on ink, the false positive rate found in the current study was considered high; with 24/30 hotspots (LS $\geq 4$ ) not having a PSM on histopathology (agreement 31\%). Comparable false positive rates are also described by Darr et al., though their workflow and CLI protocol is slightly different [11]. Several explanations for these false-positives findings can be considered. Firstly, in 19 false positives the tumour-to-margin distance was within $1 \mathrm{~mm}$ on histopathology. Due to the average positron range of $2.8 \mathrm{~mm}$ for ${ }^{68} \mathrm{Ga}$ [19], the CLI technique has inherent difficulties in the detection of PSM defined as 'tumour on ink'. If instead of tumour on ink a PSM is defined as a margin of $\leq 1 \mathrm{~mm}$, agreement between $\mathrm{CLI}$ and histopathology increases to $82 \%$. The clinical relevance of a close margin is contradicting; some argue that having a CSM results in a higher rate of biochemical recurrence compared to a NSM, though it is not an independent predictor [20]. The role of a CSM in biochemical recurrence is found by lzard et al. as well, though having a CSM definition of $<0.1 \mathrm{~mm}$ [21]. CLI might have added value to guide surgeons toward areas with an increased risks on PSM and CSM. The positron range might as well influence the correlation between the extent of the PSM on CLI and histopathology; in 5/10 PSMs the deviation between the extent on $\mathrm{CLI}$ and PA was more than $1 \mathrm{~mm}$ (Table 2). This can as well be caused by shrinkage of the specimen during fixation and histopathological processing. Still, it is worth further exploring it, as the extent of the PSM is an important prognostic factor [22].

Secondly, the presence of the bioluminescence signal also contributed to the false positive findings, especially in the base. In all included patients it was visible in the base in varying degrees, even when the primary tumour was not located there, and it was observed in non-radioactive specimens as well. The latter showed that this 
signal does not originate from ${ }^{68} \mathrm{Ga}-\mathrm{PSMA}-11$. Extensive rinsing with sodium chloride did not make the signal disappear, thus eliminating the effect of radioactive urine and blood contamination, as was initially suggested by Darr et al. [11]. Our hypothesis is that this signal is bioluminescence caused by the use of diathermy. The sudden heat from electro surgery, is known to result in the production of Reactive Oxygen Species (ROS) [23]. After a cascade of reactions, decomposition of the molecule occurs resulting in emission of optical photons. These emitted photons are within the range of 400-710 nm [24], and thus overlap partially with the Cerenkov emission spectrum. In our institute, diathermy is mainly performed when separating the prostate from the bladder, though it is occasionally also used in other areas. Therefore bioluminescence is most prevalent at the base, explaining why the correspondence between CLI (hotspot yes/no) and histopathology was only 20\% (Figure 2). However, visual interpretation with addition of a Likert score of the bioluminescence still influenced the agreement, as four PSMs at the base were false negative on CLI with a Likert score of 3, Figure 3C. In order to facilitate better detectability of PSMs a method to distinguish between Cerenkov and the diathermy-induced bioluminescence is eminent.

The mean signal-to-background ratio of the bioluminescent signal and the TBR of a PSM-suspected hotspots on unfiltered images were comparable: $4.9 \pm 1.1$ and $5.3 \pm 2.3$, respectively. A study by Spinelli et al. found a heat-induced bioluminescent half-life of 4-6 minutes in chicken breast tissue [13]. They suggested a potential distinction between Cerenkov and bioluminescence based on half-life. However, the half-life's of both signals in prostatic tissue showed overlap; the bioluminescence half-life found in this study was $48 \pm 11$ minutes compared to 68 minutes for ${ }^{68} \mathrm{Ga}$. The discrepancy in bioluminescence half-life compared with Spinelli et al. may be explained by the differences in tissue composition and methods used to induce thermal damage A focused ultrasound transducer (60 Watt) was used by Spinelli et al. [13], while in our institution a monopolar diathermy device (Intuitive, Sunnyvale, CA) at 40 Watt was used. The higher the power, the larger the temperature increase and the stronger the bioluminescent signal [23].

Another solution may be to look for alternative forms for the use of monopolar diathermy, with a lower wattage resulting in less thermal induction and thermal spread $[25,26]$, for example the PEAK PlasmaBlade (Medtronic, USA). Next, a theoretical method to reduce the presence of free radicals in tissue, and thereby preventing lipid peroxidation, could be the use of anti-oxidants like vitamin C or E [27]. Wheth- 
er these are viable solutions requires further investigation and for clinical use it is of key importance that they do not interfere with the surgical outcome or histopathological evaluation of the specimens.

To investigate an alternative method to CLI that is potentially less susceptible to bioluminescence, a flexible scintillating film was applied onto the specimen to perform FAR. The opaque film largely blocks optical photons, while positrons are detected directly. The signal originating from ${ }^{68} \mathrm{Ga}$-PSMA-11 containing areas (tumour and benign) were enhanced $\sim 3.2$ times, compared to these areas on CLI (Figure 5 \& Supplemental Figure 1), whereas bioluminescence was reduced to $70 \%$. Although this technique might help to distinguish between ${ }^{68} \mathrm{Ga}-\mathrm{PSMA}-11$ and bioluminescence, there are some practical drawbacks to consider. Firstly, the scintillating film obscures the white light image and the anatomical or spatial information of the specimen is thus absent. Secondly, accurate positioning of the scintillating film on the specimen proved challenging, and more work is needed to improve its ease of use. Lastly, there is the potential for false positives from contamination of the scintillator as shown in Figure 5.

\section{FutURE PERSPECTIVES ON CLI}

The current histopathological definition of a PSM, being tumour on ink, is a requirement that currently cannot be met with ${ }^{68} \mathrm{Ga}-P S M A-C L I$. Therefore, instead of being used as a stand-alone technology our group proposes the CLI as screening tool for using NeuroSAFE. By sending only the areas that show suspicious hotspots on the CLI image for NeuroSAFE examination, instead of the entire neurovascular bundle, the procedure may become faster. $60-75 \%$ of the PSMs occur at the apex or posterolateral side of the prostate $[22,28]$, while the bioluminescence occurs especially at the basal side. When diathermy is used in other prostate area's the surgeons are able to identify these locations visually, so inclusion of this knowledge during image interpretation may further improve the accuracy of CLI for combined use with NeuroSAFE. 


\section{Conclusion}

$\mathrm{CLI}$ of ${ }^{68} \mathrm{Ga}-\mathrm{PSMA}-11$ can be used for margin assessment during prostate cancer surgery. In the current study the agreement between CLI and histopathology was $30-82 \%$ for the entire prostate, depending on definition of PSM and application of image interpretation using the Likert scale. The best results were achieved for cancer $\leq 1 \mathrm{~mm}$ from the margin on histopathology. The presence of bioluminescence from diathermy, especially profound in the basal area, hampers image interpretation of CLI. Therefore, ${ }^{68} \mathrm{Ga}$-PSMA-11 CLI is most applicable to detect close and positive surgical margins in the apex and posterolateral side. 


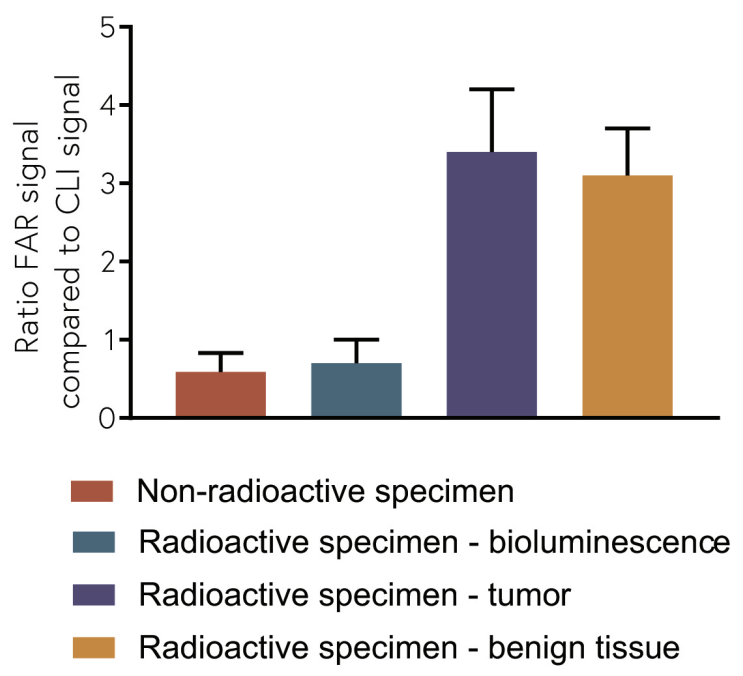

Supplemental Figure 1. A bar chart showing the effect of the application of flexible autoradiography (FAR) in different specimens. The signal is displayed as a ratio compared to the unfiltered CLI image without FAR. The FAR is applied in non-radioactive and in radioactive specimens. In the latter, the three bar charts display the signals originating from areas with tumour, benign tissue and areas where diathermy was applied. Each bar chart displays the average ratio and standard deviation measured 3 different specimens.

Supplemental Table 1. Agreement between CLI and histopathology at three regions of the prostate. The regions are divided into the number of PSMs, a tumour within $1 \mathrm{~mm}$ of the surface [CSM], a NSM [tumour $>1 \mathrm{~mm}$ away from the surface) in the analysed pathology slide. CLI is divided into both on presence of hotspots [yes/no] and categorized by Likert scores [1-3: likely NSM, 4-5: likely PSM]. The agreement between CLI and histopathology is given at every region and overall.

\begin{tabular}{|c|c|c|c|c|c|c|}
\hline & & & Base & Mid-gland & Apex & Overall \\
\hline \multirow[t]{3}{*}{ Histopathology } & PSM & & 4 & 0 & 6 & 10 \\
\hline & \multirow[t]{2}{*}{ NSM } & $>1 \mathrm{~mm}$ & 14 & 12 & 9 & 37 \\
\hline & & $\begin{array}{l}\leq 1 \mathrm{~mm} \\
\text { (CSM) }\end{array}$ & 2 & 6 & 11 & 19 \\
\hline \multirow[t]{3}{*}{ CLI hotspot } & \multirow[t]{2}{*}{ Yes } & LS: 1-3 & 19 & 3 & 3 & 25 \\
\hline & & LS: 4-5 & 1 & 6 & 23 & 30 \\
\hline & \multicolumn{2}{|l|}{ No } & 0 & 9 & 0 & 9 \\
\hline \multirow{2}{*}{$\begin{array}{l}\text { Agreement } \\
\mathrm{CLI} \text { and PA }\end{array}$} & \multicolumn{2}{|l|}{ Excl. CSM } & $20 \%$ & $50 \%$ & $23 \%$ & $31 \%$ \\
\hline & \multicolumn{2}{|l|}{ Incl. CSM } & $30 \%$ & $83 \%$ & $65 \%$ & $59 \%$ \\
\hline \multirow{2}{*}{$\begin{array}{l}\text { Agreement } \\
C L I \text { based on LS and PA }\end{array}$} & \multicolumn{2}{|l|}{ Excl. CSM } & $75 \%$ & $67 \%$ & $36 \%$ & $59 \%$ \\
\hline & \multicolumn{2}{|l|}{ Incl. CSM } & $80 \%$ & $89 \%$ & $77 \%$ & $82 \%$ \\
\hline
\end{tabular}

CSM- close surgical margin, LS- Likert score PA-Histopathology, PSM-positive surgical margin, NSM negative surgical margin. 


\section{REFERENCES}

1. Yossepowitch O, Briganti A, Eastham JA, et al. Positive Surgical Margins After Radical Prostatectomy: A Systematic Review and Contemporary Update. Eur Urol. 2014;65:303-313.

2. Stephenson AJ, Wood DP, Kattan MW, et al. Location, extent and number of positive surgical margins do not improve accuracy of predicting prostate cancer recurrence after radical prostatectomy. J Urol. 2009;182:1357-1363.

3. Silberstein J, Eastham J. Significance and management of positive surgical margins at the time of radical prostatectomy. Indian J Urol. 2014;30:423-428.

4. olde Heuvel J, de Wit-van der Veen BJ, Huizing DM, et al. State-of-the-art Intraoperative Imaging Technologies for Prostate Margin Assessment: A Systematic Review. Eur Urol Focus. 2020. [Epub ahead of print].

5. Schlomm T, Tennstedt P, Huxhold C, et al. Neurovascular structure-adjacent frozen-section examination (NeuroSAFE) increases nerve-sparing frequency and reduces positive surgical margins in open and robot-assisted laparoscopic radical prostatectomy: experience after 11,069 consecutive patients. Eur Urol. 2012;62:333-340.

6. Dinneen EP, Van Der Slot M, Adasonla K, et al. Intraoperative Frozen Section for Margin Evaluation During Radical Prostatectomy: A Systematic Review. Eur Urol Focus. 2019;6:664-673.

7. van der Slot MA, Bakker MA, Klaver S, et al. Intraoperative assessment and reporting of radical prostatectomy specimens to guide nerve-sparing surgery in prostate cancer patients (NeuroSAFE). Histopathology. 2020;77:539-547.

8. Grootendorst MR, Cariati M, Pinder SE, et al. Intraoperative Assessment of Tumour Resection Margins in Breast-Conserving Surgery Using ${ }^{18}$ F-FDG Cerenkov Luminescence Imaging: A Firstin-Human Feasibility Study. J Nucl Med. 2017;58:891-898.

9. Ciarrocchi E, Belcari N. Cerenkov luminescence imaging: physics principles and potential applications in biomedical sciences. EJNMMI Phys. 2017;4:14.

10. Robertson R, Germanos MS, Li C, et al. Optical imaging of Cerenkov light generation from positron-emitting radiotracers. Phys Med Biol. 2009;54:N355-65.

11. Darr C, Harke N, Radtke JP, et al. Intraoperative ${ }^{68}$ Gallium-PSMA Cerenkov Luminescence Imaging for surgical margins in radical prostatectomy - a feasibility study. J Nucl Med. 2020;61:1500-1506.

12. olde Heuvel J, de Wit-van der Veen BJ, van der Poel HG, et al. ${ }^{68} \mathrm{Ga}-P S M A$ Cerenkov luminescence imaging in primary prostate cancer: first-in-man series. Eur J Nucl Med Mol Imaging. 2020;47:2624-2632

13. Spinelli AE. Weak light emission of soft tissues induced by heating. J Biomed Opt. 2018; 23:046003.

14. Boschi F, Basso PR, Corridori l, et al. Weak biophoton emission after laser surgery application in soft tissues: Analysis of the optical features. J Biophotonics. 2019;12:e201800260.

15. Ciarrocchi E, Belcari N. Cerenkov luminescence imaging: physics principles and potential applications in biomedical sciences. EJNMMI Phys. 2017;4:14.

16. Vyas KN, Grootendorst M, Mertzanidou T, et al. Flexible scintillator autoradiography for tumour margin inspection using ${ }^{18}$ F-FDG. Proc. SPIE 10478, Molecular-Guided Surgery: Molecules, Devices, and Applications IV, 1047811. 2018

17. Samaratunga H, Montironi R, True L, et al. International Society of Urological Pathology(ISUP) Consensus Conference on Handling and Staging of Radical Prostatectomy Specimens. Work- 
ing group 1: specimen handling. Mod Pathol. 2011;24:6-15.

18. Pukelsheim F. The Three Sigma Rule. Am Stat. 1994;48:88-91.

19. Moses WW. Fundamental limits of spatial resolution in PET. Nucl Instruments Methods Phys Res Sect A. 2011;648:S236-S240.

20. Herforth C, Stroup SP, Chen Z, et al. Radical prostatectomy and the effect of close surgical margins: results from the Shared Equal Access Regional Cancer Hospital (SEARCH) database. BJU Int. 2018;122:592-598.

21. Izard JP, True LD, May P, et al. Prostate Cancer That Is Within $0.1 \mathrm{~mm}$ of the Surgical Margin of a Radical Prostatectomy Predicts Greater Likelihood of Recurrence. Am J Surg Pathol. 2014;38:333-338.

22. Koskas $\mathrm{Y}$, Lannes $\mathrm{F}$, Branger $\mathrm{N}$, et al. Extent of positive surgical margins following radical prostatectomy: Impact on biochemical recurrence with long-term follow-up. BMC Urol. 2019;19:1-8.

23. Kobayashi K, Okabe H, Kawano S, et al. Biophoton Emission Induced by Heat Shock. PLoS One. 2014;9:e105700.

24. D. Fels, M. Cifra and F. Scholkmann, Fields of the Cell, 2015 In: Prasad A, Pospišil P. The photon source within the cell. Research Signpost. 2015:113-129

25. Lyons SD, Law KSK. Laparoscopic Vessel Sealing Technologies. J Minim Invasive Gynecol. 2013;20:301-307.

26. Hefermehl LJ, Largo RA, Hermanns T, et al. Lateral temperature spread of monopolar, bipolar and ultrasonic instruments for robot-assisted laparoscopic surgery. BJU Int. 2014;114:245-252.

27. Catala A. Lipid peroxidation. In: Repetto M, Semprine J, Boveris A. Lipid Peroxidation: Chemical Mechanism, Biological Implications and Analytical Determination. IntechOpen, 2012:3-30.

28. Eastham JA, Kuroiwa K, Ohori M, et al. Prognostic Significance of Location of Positive Margins in Radical Prostatectomy Specimens. Urology. 2007;70:965-969. 



\section{CHAPTER 8}

GENERAL DISCUSSION AND FUTURE PERSPECTIVES 


\section{General discussion and future perspectives}

The application of specific tumour radiotracers in biomedical imaging has expanded in the last decade. For patients with primary prostate cancer (PCa), PET/CT imaging with gallium-68 $\left({ }^{68} \mathrm{Ga}\right)$ coupled prostate specific membrane antigen (PSMA) in the primary setting has progressed enormously. Besides in diagnostics, this tracer can nowadays also be used for PSMA-directed radio-guided prostatectomy, as was demonstrated in this thesis. The goal of surgery is to ensure a radical removal of the cancer, with minimal damage to surrounding tissues. However, positive surgical margins (PSM) occur in up to $40 \%$ of patient with PCa, depending on the stage of the cancer [1-3]. Despite contradicting literature about the correlation of PSM with a biochemical recurrence (BCR), the evident goal is to reduce the PSM rate and limit the additional treatments a patient has to undergo. Intraoperative margin assessment using PSMA-based Cerenkov luminescence imaging (CLI) might contribute to this goal. Therefore, the aim of the research presented in this thesis was to investigate the feasibility of ${ }^{68} \mathrm{Ga}-\mathrm{PSMA}-11 \mathrm{CLI}$ as intraoperative margin assessment technology in prostate cancer surgery.

In this chapter, the findings described in this thesis and the corresponding clinical consequences are discussed, both for ${ }^{68} \mathrm{Ga}$-PSMA-11 PET/CT and CLI. Furthermore, future prospects for the use of PSMA PET/CT in patients with primary PCa and requirements for clinical use of CLI for margin assessment during prostatectomy are described. Finally, a glimpse into the future of image guided surgery with respect to margin assessment and the utilization of CLI in other types of cancer is given.

\section{${ }^{68}$ GA-PSMA-11 PET/CT FOR CLI OPTIMIZATION}

In daily clinical practice, PSMA-PET/CT is a diagnostic tool for staging primary PCa with high sensitivity and specificity [4]. The usability of PSMA-tracers is currently expanding towards intraoperative margin assessment. In this respect, pre-operative information provided by the diagnostic ${ }^{68} \mathrm{Ga}$-PSMA-11 PET/CT may be used for preoperative guidance, provided that lesion accumulation profiles are stable over time. To address this issue, the repeatability of ${ }^{68} \mathrm{Ga}-\mathrm{PSMA}-11$ uptake in a four week interval was investigated in Chapter 4 . The study concluded that the uptake was repeatable within this time interval, indicating that indeed ${ }^{68} \mathrm{Ga}$-PSMA-11 uptake on pre-operative PET/CT serves as an indicator for intraoperative accumulation. 
With intraoperative imaging, the main interest is sufficient contrast to discriminate primary tumour from benign tissue in the prostate. Even so, more practical issues such as timing of the injection and image acquisition are of importance given the relative short half-life of ${ }^{68} \mathrm{Ga}$. For ${ }^{68} \mathrm{Ga}-\mathrm{PSMA}-11 \mathrm{PET} / \mathrm{CT}$, the European guidelines indicate an uptake time of $60 \mathrm{~min}$ (range 50-100 min) [5], in which an sufficient tumour-to-background contrast can be reached. However, the underlying evidence for this time-interval is limited [6]procedures have been developed to label PSMA ligands with (68, and there are publications that promote early time-point imaging to achieve a higher tumour-contrast $[7,8]$. Therefore, the uptake kinetics of ${ }^{68} \mathrm{Ga}$-PSMA-11 were assessed using dynamic PET of the pelvic area in Chapter 5. Based on these results, the timing of the ${ }^{68} \mathrm{Ga}-\mathrm{PSMA}-11$ injection with respect to the start of surgery was identified. Though, the tumour-to-background ratio (TBR) increased over time, the TBR was already sufficient after 5 minutes for CLI. It was concluded that for intraoperative $\mathrm{CLI}$ a large time window is feasible for imaging. As timing in the operation room is difficult to plan in advance, the flexibility of this injection timing is an advantage. Nevertheless, one should keep the half-life of ${ }^{68} \mathrm{Ga}$ ( $67.7 \mathrm{~min}$ ) in mind when surgery is delayed for a longer time, as the decay affects the overall CLI signal intensity.

The dynamic study in Chapter $\mathbf{5}$ did not solely provide information relevant for CLI study, but also showed an unexpected finding for ${ }^{68} \mathrm{Ga}-\mathrm{PSMA}-11 \mathrm{PET} / \mathrm{CT}$ in general; two very distinct tumour accumulation patterns (i.e., stable and rising) were observed. The current hypothesis is that this distinction can be explained by differences in tumour volume, where in large volumes $(\sim 30 \mathrm{cc})$ the uptake rises over 60 minutes, whereas in small volumes $(\sim 3 \mathrm{cc})$ the uptake stabilizes 5 minutes post injection. It was hypothesized that this dependence of accumulation on tumour load might provide details on tumour phenotype and/or receptor density. However, this study was only conducted in a small group $(n=5)$ and the current theory is not supported by previous literature, thus a larger study into the early dynamics of ${ }^{68} \mathrm{Ga}-\mathrm{PSMA}-11$ is warranted.

\section{FUTURE PERSPECTIVES FOR ${ }^{68}$ GA-PSMA-11 PET/CT}

In this thesis, we did not assess the relation between tumour uptake on PET and $\mathrm{CLI}$, since such a relationship is well described in literature [9]. Combining this imaging data, it might be used for more structured and reliable identification of the PSMs on CLI. However, for a good correlation between the uptake on PSMA-PET/CT and 
the CLI signal, reliable segmentation is imminent. At this moment in time, there is no consensus on a standardized methodology for tumour segmentation in PSMA-PET/ CT [10-13]. On top of that, it is challenging to segment the prostate tumour on anatomical imaging, due to the lack of soft tissue contrast on CT. This specific problem was encountered in Chapter $\mathbf{4}$ as well, when measuring the average accumulation and tumour volume. Therefore, we notice that studies on accurate lesion segmentation by using deep-learning [14], and semi-automatic software tools [15] are highly relevant. Another option to improve segmentation is to use PET/MRI, as MRI has a better soft tissue contrast. In addition, these complementary techniques provide a high sensitivity of PET/MRI for detection of primary PCa, despite the long acquisition time and the need for scatter correction [16].

Ideally, future research on dynamic PET will be conducted with novel so-called total body PET-scanners to enable kinetic evaluation of multiple organs simultaneously $[17,18]$. Since ${ }^{68} \mathrm{Ga}-\mathrm{PSMA}-11$ kinetics can be described as an irreversible two compartment model [19], it might be interesting to look at the added value of total body Patlak modelling [20]. The $\mathrm{K}_{\mathrm{i}}$ value for example, denoting the influx rate constant, can be visualised as a time-weighted signal, resulting in 3D-images that are quite 162 comparable to the standard SUV images [20], Figure 1. In receptor imaging, these kinetic evaluations provide additional information on for instance tumour receptor density and cellular targeting, thereby aiding tumour identification.

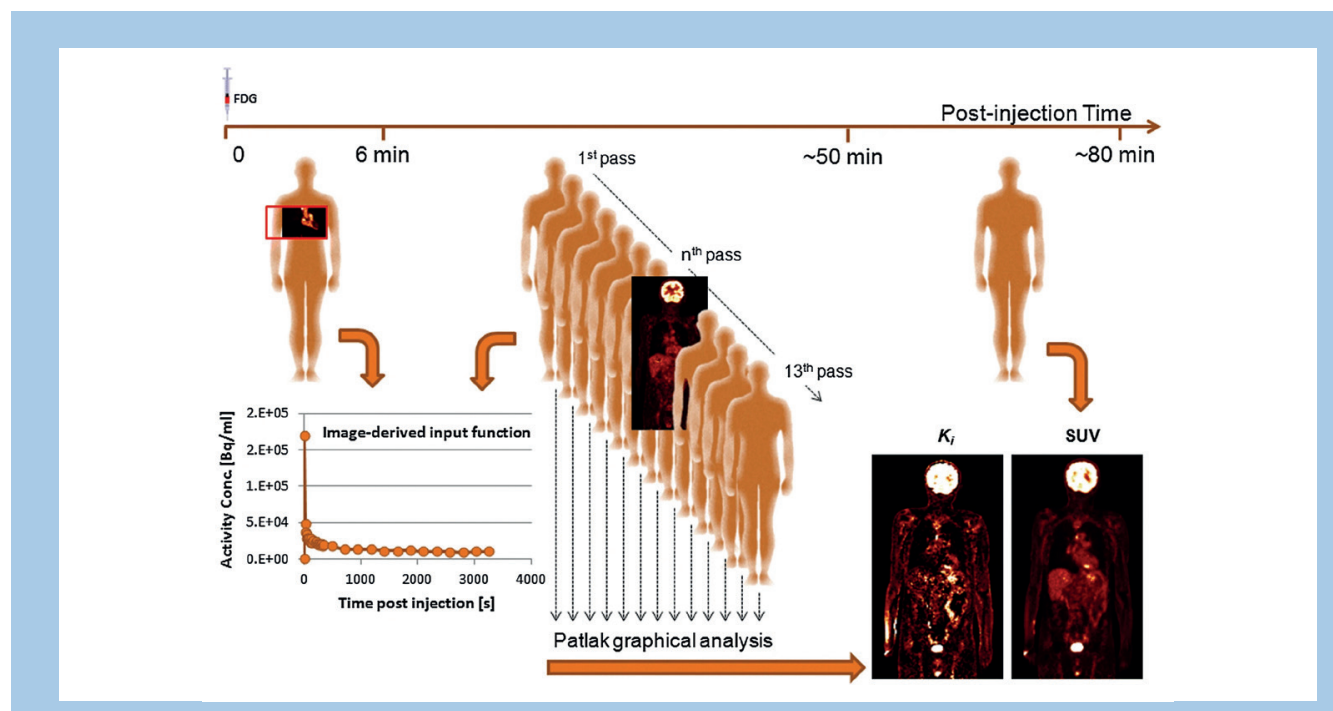

Figure 1. Example of $K_{i}$ and SUV imaging of a total body FDG PET/CT. Obtained from Fahrni et al. [20], with permission. 
With the knowledge obtained in Chapter 4 \& 5 regarding the repeatability and tracer kinetics for ${ }^{68} \mathrm{Ga}-\mathrm{PSMA}-11$, and the in vitro $\mathrm{CLI}$ results in Chapter $\mathbf{3}$, the workflow for the clinical CLI study was designed. The feasibility of this workflow for CLI margin assessment in prostate cancer surgery was assessed in Chapter 6 . Based on these initial results, the acquisition protocol was further optimized for ex vivo usage, and the radiation exposure to staff was considered acceptable. This positive verdict on the technical feasibility led to a continuation of the main study

\section{in Chapter 7.}

Eventually, CLI margin assessment was performed in a total of 15 patients. The accuracy in comparison to histopathology was assessed in all patients and the newly identified bioluminescence signal was characterized. We showed that CLI was able to accurately detect tumour lesions close to the surface $(\leq 1 \mathrm{~mm})$ in $82 \%$ of the cases. However, when purely considering conventional PSM, defined as tumour on ink, we encountered a relatively high false positive rate for $\mathrm{CLI}$. This is probably caused by two reasons; first the depth sensitivity of CLI, and secondly, the presence of an additional bioluminescence signal.

Depth sensitivity of CLI

In medicine, new techniques are generally compared to the 'gold standard'. The main difficulty in prostate cancer is the specific definition of a PSM on histopathology, which is based on one cell layer on histopathology (tumour on ink). Being realistic, such an depth accuracy will be very difficult to achieve by any optical imaging technology, as was also demonstrated in Chapter 2. For CLI, the positron range of the used isotope determines the final depth sensitivity that can be reached. In our case, the positron range of ${ }^{68} \mathrm{Ga}$ is on average $2.8 \mathrm{~mm}$, which is far from ideal. One may argue about the histopathological definition of PSM, as some suggest that a close margin (tumour within $1 \mathrm{~mm}$ from the specimen's border) increases the risk of BCR compared to a NSM $[21,22]$. For now, the detection of a close margin seems to be the maximum achievable with CLI.

Nevertheless, CLI has advantages that even in its current form, outweigh histopathology. In histopathology, the prostate is cut into sections of $\sim 3 \mathrm{~mm}$, and of every section one histology slide ( $3-5 \mu \mathrm{m}$ thick) is assessed by the pathologist, thus causing undersampling. In contrast, CLI has the possibility to assess the entire surface of the prostate, and guide the pathologist towards regions at risk. Therefore, we expect that there are potentially valuable clinical applications for CLI [23], especial- 
ly in combination with other technologies.

\section{Bioluminescence}

Unfortunately, Chapter 7 showed that Cerenkov is not the only light that shines. In most patients, including those without any ${ }^{68} \mathrm{Ga}-P S M A-11$ injection, an intense signal was observed profoundly at the base of the resected prostate, even though no tumour was present there. This meant that this signal did not originate from ${ }^{68} \mathrm{Ga}$-PSMA-11, but it was hypothesized to be diathermy induced bioluminescence. It was outside the scope of this thesis to determine the chemical origin and physics of this bioluminescence. The effects of for example a higher diathermy power, a longer duration of tissue contact and influence of tissue composition should be investigated in prostatic tissue to better understand the signal. Prior studies were mostly conducted in chicken breast tissue [24,25], without any perfusion.

Fortunately, diathermy is mainly used at the base of the prostate, which is a location where the PSM incidence is low. Furthermore, in case a PSM does exist on the base of the prostate, there is almost no opportunity to resect additional tissue, as the base is in close contact with the bladder neck. Dissection near the bladder neck would result in severe functional impairments, outweighing the necessity of a radical resection. The apex and mid-prostate are less affected by diathermia and CLI interpretation is therefore less likely to be influenced by this bioluminescence signal.

\section{FUTURE PERSPECTIVES FOR INTRAOPERATIVE MARGIN ASSESSMENT}

\section{$C L I$ in combination with other techniques}

A promising application might be to combine CLI with the NeuroSAFE technology [26-28], which is shown in Figure 2. NeuroSAFE is a perioperative histopathological procedure, in which the PSM rate decreased $6.5 \%$ to $14.5 \%$ compared to the PSMrate in patients without NeuroSAFE [29]. However, the procedure is time consuming (at least 45 minutes), and only the prostate surface at the neurovascular bundles (NVB) is assessed. So, in the NeuroSAFE procedure the apex is not assessed, while that area is prone to PSMs. Prior to inking and fixation of the prostate for histopathology, CLI may indicate areas at risk for PSM. The pathologist then only needs to assess the selected regions, thus reducing the time of the procedure. The strong negative predictive value of CLI has clear benefits, ensuring a high prediction for negative margins. If successful, nerve sparing (NS) surgery can be offered to more patients as the procedure becomes more efficient. Currently, only a limited number of patients can be selected for NS surgery in the Antoni van Leeuwenhoek, based 
on a high preoperative erectile function, organ confined prostate cancer (<cT3), and tumour location not near the NVB.

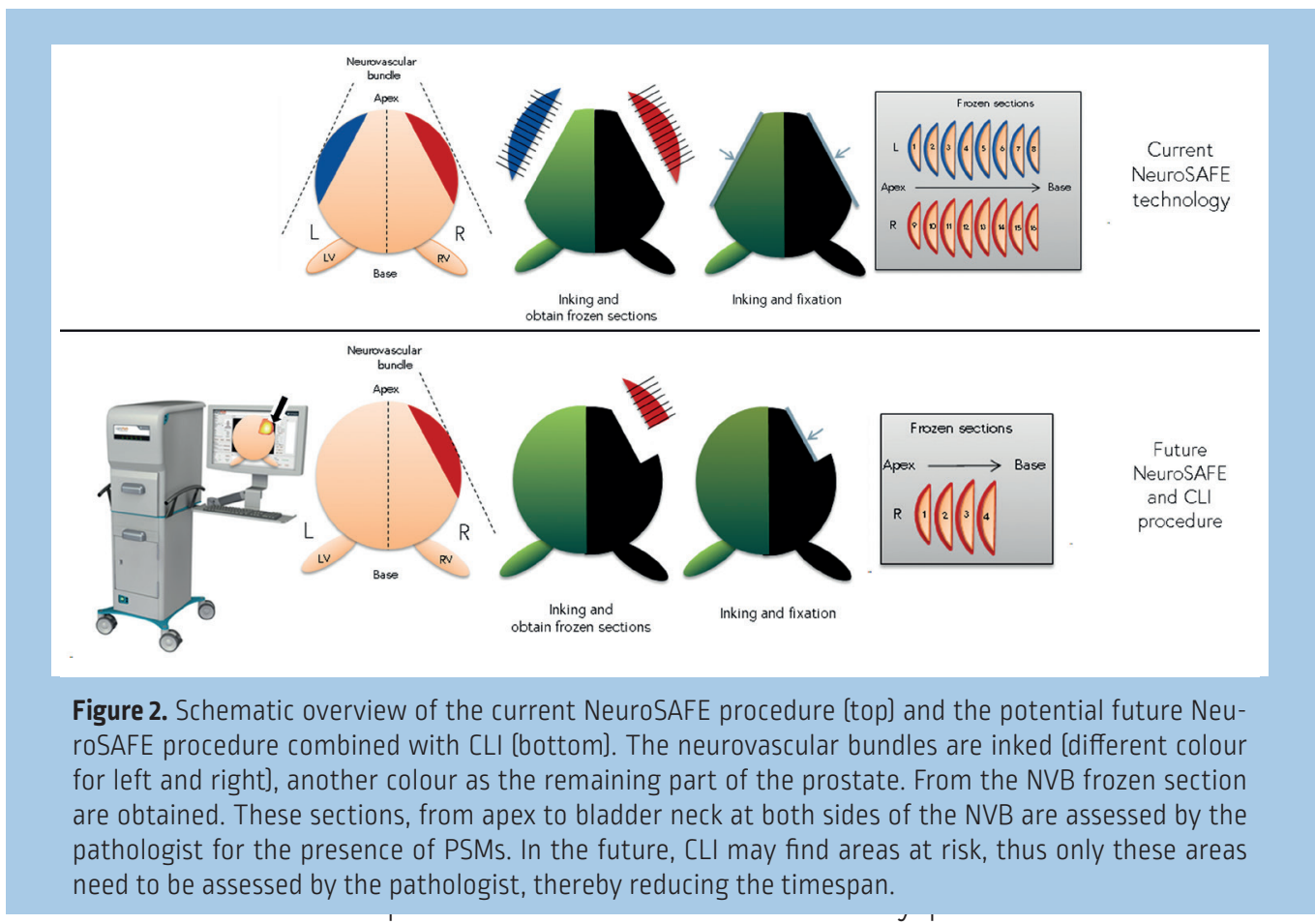

setting. As a result, the surgeon has to reorient this location back in the abdominal cavity in order to remove additional tissue if a location is suggested to be a PSM. This is burdensome because retrieving the precise location can be difficult. In vivo CLI assessment allows for more precise mapping of the PSM locations. Next, the entire surgical cavity can be scanned, in order to visualize potential residual tumour tissue, Figure 3. Cerenkov Luminescence Endoscopy (CLE) is already being performed, though not yet in prostate cancer [30-32]. 


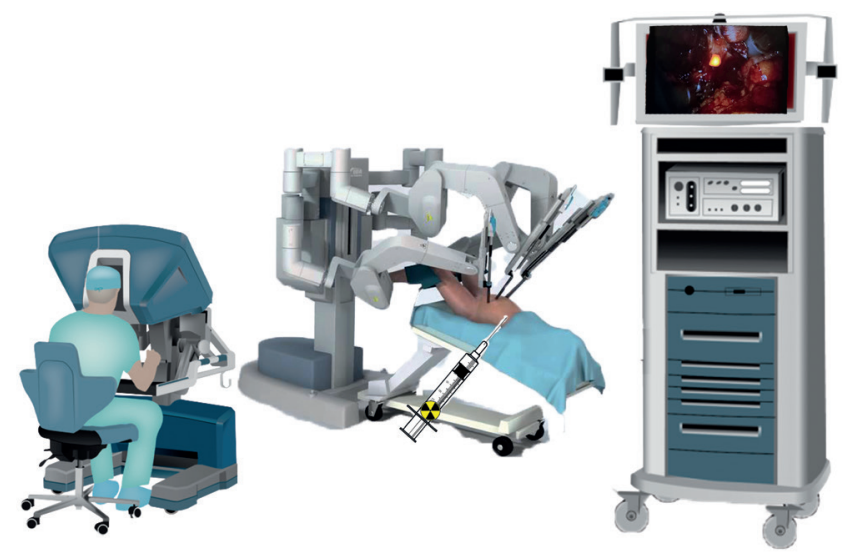

Figure 3. Potential set up for CLE, radioactivity is administered to the patient. The hotspot on the monitor represents tumour in the surgical cavity, which than can be removed. Image is adapted from [33,34].

of PSMs, in order to eventually reduce the rate of biochemical recurrence (BCR). In current clinical practice, approximately $30 \%$ of all men undergoing a prostatectomy eventually will develop a BCR $[35,36]$. If margin assessment reduces the PSM rate, and thus also the number of patients with $B C R$, it might induce a benefit for both patient outcome and healthcare costs. However, this expectation should be tempered; in a recent review assessing the NeuroSAFE procedure, the reduction in PSM rate was not related to a better overall oncological outcome [30]. In this review, three studies reported on oncological outcomes with a maximum of 5 year follow-up $[26,27,37]$. These follow-up times are quite short, given that the median time to progression is 7 years, and about half of the patients will not present a BCR until 10 years after prostatectomy [38]. So, to properly assess the added value of $\mathrm{CLI}$-alone or NeuroSAFE combined with $\mathrm{CLI}$ on patient outcome will require studies with long term follow-up. If CLI, or radio-guided surgery in general, does not provide an added value, the question has to be raised if the technique should be applied at all. The radiation exposure to the patient and the surgical staff should be kept to a minimum according to the principles of ALARA (as low as reasonably achievable).

Even though statements above might question the necessity of margin assessment, it is still acknowledged that margin assessment may be of added value for two reasons. First, it can reassure surgeons and patients to know there is no PSM present. Furthermore, the extent of a PSM is correlated to the chance of developing a BCR $[39,40]$. Patients with a PSM with an extent $>3 \mathrm{~mm}$ or several positive margins have an earlier BCR, thus qualifying for early adjuvant treatment. Whereas in case of a 
focal (single) PSM it seems legitimate to only monitor patients [39,40]. In Chapter 7 we also briefly looked at the correlation between the extent of the PSM on CLI and histopathology, with promising results.

\section{$C L I$ in other types of oncological surgery}

The acquired knowledge in this thesis regarding CLI during prostatectomy is generating hypotheses for the use of CLI in other types of oncological surgery. In tumour types that have a more favourable histopathological margin definition for CLI, the search for a tumour specific tracer can start. Main factors to take into account are a sufficient energy level to induce Cerenkov, on-site production or delivery, and half-life compatible with surgery. CLI using ${ }^{18} \mathrm{~F}-\mathrm{FDG}$ is already applied in clinical research setting in breast cancer [41]. Margin assessment may also have potential in oncological resection of head and neck cancers, with the main focus on oral cavity malignancies. ${ }^{18} \mathrm{~F}-\mathrm{FDG}$ is sensitive enough to detect oral carcinoma, however, lacks specificity, which is needed for CLI imaging. Zirconium-89 ${ }^{89} \mathrm{Zr}$ ) labelled anti-bodies [42,43] can be an option as well as the Cerenkov yield of ${ }^{89} \mathrm{Zr}$ is comparable to that of ${ }^{18} \mathrm{~F}$ [44]. The first steps regarding the use of $\mathrm{CLI}$ in pulmonary and hepatic metastasectomy using ${ }^{18} \mathrm{~F}-\mathrm{FDG}$ and ${ }^{68} \mathrm{Ga}$-DOTATOC respectively, have recently been undertaken [45].

\section{Alternatives for CLI}

Looking at alternative margin assessment tools in prostate cancer surgery, as described in Chapter 2, one cannot ignore the potential of fluorescence technology. There are some important differences between CLI and fluorescence imaging. First, the CLI signal reduces proportionally to the radioactive decay, whereas fluorescence has long biological wash out periods. Secondly, only a few clinically approved fluorescent contrast agents are currently available, whereas CLI can use multiple approved radiotracers [46]. Additionally, it proved difficult for a long time to bind fluorescent agents to tumour targeted probes. However, recently new tumour specific probes have been developed, one of them being a PSMA targeted fluorescent agents [4749]. In the Netherlands Cancer Institute, an ongoing clinical trial (EudraCT Number: 2019-002393-31) evaluates OTL-78, a PSMA-targeted fluorescent agent, for intraoperative imaging of prostate cancer. In this study, the fluorescence signal is assessed both on the specimen surface and within the surgical cavity. The histopathological definition for PSM could also be a bottleneck in fluorescence, as the depth sensitivity of this technology is questionable. Nonetheless, this aspect plays a less important role when examining the surgical cavity. 


\section{Conclusion}

In conclusion, this thesis describes the possible value of CLI during prostate cancer surgery and shows that it has potential in highlighting areas at risk of PSMs. CLI might have profound clinical impact in other types of oncological surgery, taking in consideration a proper tumour to background ratio, depth sensitivity within the range of the PSM definition and the ability for intraoperative use. The choice of radiopharmaceutical influences the $\mathrm{CLI}$ accuracy, regarding the penetration depth and signal yield. Next, the injected activity should be within an acceptable range regarding radiation exposure to patients and the clinical team. Finally, preoperative imaging gives essential information involving lesion uptake and location. As with every new complicated medical technology, implementation depends on teamwork and comes with hurdles. After a learning curve, CLI might light the path to better precise cancer treatment. 


\section{REFERENCES}

1. Stephenson AJ, Wood DP, Kattan MW, et al. Location, Extent and Number of Positive Surgical Margins Do Not Improve Accuracy of Predicting Prostate Cancer Recurrence After Radical Prostatectomy. J Urol. 2009;182:1357-1363.

2. Yossepowitch $O$, Briganti A, Eastham JA, et al. Positive Surgical Margins After Radical Prostatectomy: A Systematic Review and Contemporary Update. Eur Urol. 2014;65:303-313.

3. Evans SM, Millar JL, Frydenberg M, et al. Positive surgical margins: Rate, contributing factors and impact on further treatment: Findings from the Prostate Cancer Registry. BJU Int. 2014;114:680-690.

4. Hofman MS, Lawrentschuk N, Francis RJ, et al. Prostate-specific membrane antigen PET-CT in patients with high-risk prostate cancer before curative-intent surgery or radiotherapy (proPSMA): a prospective, randomised, multicentre study. Lancet. 2020;395:1208-1216.

5. Fendler WP, Eiber M, Beheshti M, et al. ${ }^{68}$ Ga-PSMA PET/CT: Joint EANM and SNMMI procedure guideline for prostate cancer imaging: version 1.0. Eur J Nucl Med Mol Imaging. 2017;44:10141024.

6. Afshar-Oromieh A, Malcher A, Eder M, et al. PET imaging with a $\left[{ }^{68} \mathrm{Ga}\right] g a l l i u m-l a b e l l e d ~ P S M A$ ligand for the diagnosis of prostate cancer: biodistribution in humans and first evaluation of tumour lesions. Eur J Nucl Med Mol Imaging. 2013;40:486-495.

7. Kabasakal L, Demirci E, Ocak M, et al. Evaluation of PSMA PET/CT imaging using a ${ }^{68}$ Ga-HBED$\mathrm{CC}$ ligand in patients with prostate cancer and the value of early pelvic imaging. Nucl Med Commun. 2015;36:582-587.

8. Uprimny C, Kroiss AS, Decristoforo C, et al. Early dynamic imaging in ${ }^{68} \mathrm{Ga}-\mathrm{PSMA}-11 \mathrm{PET} / \mathrm{CT}$ allows discrimination of urinary bladder activity and prostate cancer lesions. Eur J Nucl Med Mol Imaging. 2017;44:765-775.

9. Grootendorst MR, Cariati M, Kothari A, et al. Cerenkov luminescence imaging (CLI) for image-guided cancer surgery. Clin Transl Imaging. 2016;4:353-366.

10. Zamboglou C, Schiller F, Fechter T, et al. (68)Ga-HBED-CC-PSMA PET/CT Versus Histopathology in Primary Localized Prostate Cancer: A Voxel-Wise Comparison. Theranostics. 2016;6:16191628.

11. Zamboglou C, Fassbender TF, Steffan $L$, et al. Validation of different PSMA-PET/CT-based contouring techniques for intraprostatic tumor definition using histopathology as standard of reference. Radiother Oncol. 2019;141:208-213.

12. Giesel FL, Sterzing F, Schlemmer HP, et al. Intra-individual comparison of (68)Ga-PSMA-11PET/CT and multi-parametric MR for imaging of primary prostate cancer. Eur J Nucl Med Mol Imaging. 2016;43:1400-1406.

13. Thomas L, Kantz S, Hung A, et al. (68)Ga-PSMA-PET/CT imaging of localized primary prostate cancer patients for intensity modulated radiation therapy treatment planning with integrated boost. Eur J Nucl Med Mol Imaging. 2018;45:1170-1178.

14. Leung K, Ashrafinia S, Sadaghiani MS, et al. A fully automated deep-learning based method for lesion segmentation in ${ }^{18} \mathrm{~F}-\mathrm{DCFP}$ L PSMA PET images of patients with prostate cancer. J Nucl Med. 2019;60:399-399.

15. Gafita A, Bieth M, Krönke M, et al. QPSMA: Semiautomatic software for whole-body tumor burden assessment in prostate cancer using ${ }^{68}$ Ga-PSMA11 PET/CT. J Nucl Med. 2019;60:12771283.

16. Evangelista L, Zattoni F, Cassarino G, et al. PET / MRI in prostate cancer : a systematic review 
and meta-analysis. Eur J Nucl Med Mol Imaging. 2020.[Epub ahead of print]

17. Badawi RD, Shi H, Hu P, et al. First human imaging studies with the explorer total-body PET scanner. J Nucl Med. 2019;60:299-303.

18. Vandenberghe S, Moskal P, Karp JS. State of the art in total body PET. EJNMMI Phys. 2020;7:35.

19. Ringheim A, Campos Neto G de C, Anazodo U, et al. Kinetic modeling of ${ }^{68} \mathrm{Ga}-\mathrm{PSMA}-11$ and validation of simplified methods for quantification in primary prostate cancer patients. EJNMMI Res. 2020;10:12.

20. Fahrni G, Karakatsanis NA, Di Domenicantonio G, et al. Does whole-body Patlak ${ }^{18}$ F-FDG PET imaging improve lesion detectability in clinical oncology? Eur Radiol. 2019;29:4812-4821.

21. Herforth C, Stroup SP, Chen Z, et al. Radical prostatectomy and the effect of close surgical margins: results from the Shared Equal Access Regional Cancer Hospital (SEARCH) database. BJU Int. 2018;122:592-598.

22. Whalen MJ, Shapiro EY, Rothberg MB, et al. Close surgical margins after radical prostatectomy mimic biochemical recurrence rates of positive margins. Urol Oncol Semin Orig Investig. 2015;33:494.e9-494.e14.

23. Bagguley D, Cumberbatch M, Lawrentschuk N, Murphy DG. Cerenkov Luminescence Imaging for surgical margins in radical prostatectomy: a surgical perspective. J Nucl Med. 2020;61:14981499.

24. Spinelli AE. Weak light emission of soft tissues induced by heating. J Biomed Opt. 2018;23:1-5.

25. Boschi F, Basso PR, Corridori I, et al. Weak biophoton emission after laser surgery application in soft tissues: Analysis of the optical features. J Biophotonics. 2019;12: e201800260.

170 26. Schlomm T, Tennstedt P, Huxhold C, et al. Neurovascular structure-adjacent frozen-section examination (NeuroSAFE) increases nerve-sparing frequency and reduces positive surgical margins in open and robot-assisted laparoscopic radical prostatectomy: experience after 11,069 consecutive patients. Eur Urol. 2012;62:333-340.

27. Mirmilstein G, Rai BP, Gbolahan $\mathrm{O}$, et al. The neurovascular structure-adjacent frozen-section examination (NeuroSAFE) approach to nerve sparing in robot-assisted laparoscopic radical prostatectomy in a British setting - a prospective observational comparative study. BJU Int. 2018;121:854-862.

28. Beyer B, Schlomm T, Tennstedt P, et al. A Feasible and Time-efficient Adaptation of NeuroSAFE for da Vinci Robot-assisted Radical Prostatectomy. Eur Urol. 2014;66:138-144.

29. Dinneen EP, Van Der Slot M, Adasonla K, et al. Intraoperative Frozen Section for Margin Evaluation During Radical Prostatectomy: A Systematic Review. Eur Urol Focus. 2019;6:664-673.

30. Hu H, Cao X, Kang F, et al. Feasibility study of novel endoscopic Cerenkov luminescence imaging system in detecting and quantifying gastrointestinal disease: first human results. Eur Radiol. 2015;25:1814-1822.

31. Zhang Z, Cai M, Bao C, et al. Endoscopic Cerenkov luminescence imaging and image-guided tumor resection on hepatocellular carcinoma-bearing mouse models. Nanomedicine Nanotechnology, Biol Med. 2019;17:62-70.

32. Liu H, Carpenter CM, Jiang H, et al. Intraoperative Imaging of Tumors Using Cerenkov Luminescence Endoscopy: A Feasibility Experimental Study. J Nucl Med. 2012;53:1579-1584.

33. High Impact. Robotic Assisted Laparoscopic Prostatectomy [Internet]. 2020 [assessed on 2020 Nov 13]. Available from: https://highimpact.com/exhibits/robotic-assisted-laparoscopic-prostatectomy 
34. Ehealth Network. Robotic heart surgery [Internet]. 2018 [assessed on 2020 Nov 13]. Available from:https://ehealth.eletsonline.com/2018/12/in-a-first-gujarat-doctor-performsworlds-first-robotic-heart-surgery/

35. Pompe RS, Gild P, Karakiewicz Pl, et al. Long-term cancer control outcomes in patients with biochemical recurrence and the impact of time from radical prostatectomy to biochemical recurrence. Prostate. 2018;78:676-681.

36. Tilki D, Preisser F, Graefen M, et al. External Validation of the European Association of Urology Biochemical Recurrence Risk Groups to Predict Metastasis and Mortality After Radical Prostatectomy in a European Cohort. Eur Urol. 2019;75:896-900.

37. Lavery HJ, Xiao G-Q, Nabizada-Pace F, et al. "Mohs surgery of the prostate": the utility of in situ frozen section analysis during robotic prostatectomy. BJU Int. 2011;107:975-979.

38. Swanson GP, Riggs MW, Earle JD, et al. Long-term follow-up of radical retropubic prostatectomy for prostate cancer. Eur Urol. 2002;42:212-216.

39. Koskas $Y$, Lannes $F$, Branger $N$, et al. Extent of positive surgical margins following radical prostatectomy: Impact on biochemical recurrence with long-term follow-up. BMC Urol. 2019;19:1-8.

40. Martini A, Gandaglia G, Fossati N, et al. Defining Clinically Meaningful Positive Surgical Margins in Patients Undergoing Radical Prostatectomy for Localised Prostate Cancer. Eur Urol Oncol. 2019. [Epub ahead of print].

41. Grootendorst MR, Cariati M, Pinder SE, et al. Intraoperative Assessment of Tumor Resection Margins in Breast-Conserving Surgery Using ${ }^{18} \mathrm{~F}-F D G$ Cerenkov Luminescence Imaging: A Firstin-Human Feasibility Study. J Nucl Med. 2017;58:891-898

42. Zhao S, Pan W, Jiang $\mathrm{H}$, et al. Cerenkov luminescence imaging is an effective preclinical tool for assessing colorectal cancer PD-L1 levels in vivo. EJNMMI Res. 2020;10:64.

43. D'Souza JW, Hensley H, Doss M, et al. Cerenkov luminescence imaging as a modality to evaluate antibody-based pet radiotracers. J Nucl Med. 2017;58:175-180.

44. Gill RK, Mitchell GS, Cherry SR. Computed Cerenkov luminescence yields for radionuclides used in biology and medicine. Phys Med Biol. 2015;60:4263-4280.

45. Ciarrocchi E, Saponaro S, Bartoli F, et al. Preliminary study of the feasibility of Cerenkov luminescence imaging in metastasectomy. Annual Congress of the European Association of Nuclear Medicine 2020

46. Ciarrocchi E, Belcari N. Cerenkov luminescence imaging: physics principles and potential applications in biomedical sciences. EJNMMI Phys. 2017;4:14.

47. Derks YHW, Löwik DWPM, Sedelaar JPM, et al. PSMA-targeting agents for radio- And fluorescenceguided prostate cancer surgery. Theranostics. 2019;9:6824-6839.

48. van Leeuwen PJ, van Oosterom MN, de Barros H, et al. Prostate specific membrane antigen as target for surgical guidance. Tijdschr voor Urol. 2020;10:124-130.

49. Maurer T, Graefen M, van der Poel H, et al. Prostate-specific membrane antigen - Guided surgery. J Nucl Med. 2020;61:6-12 


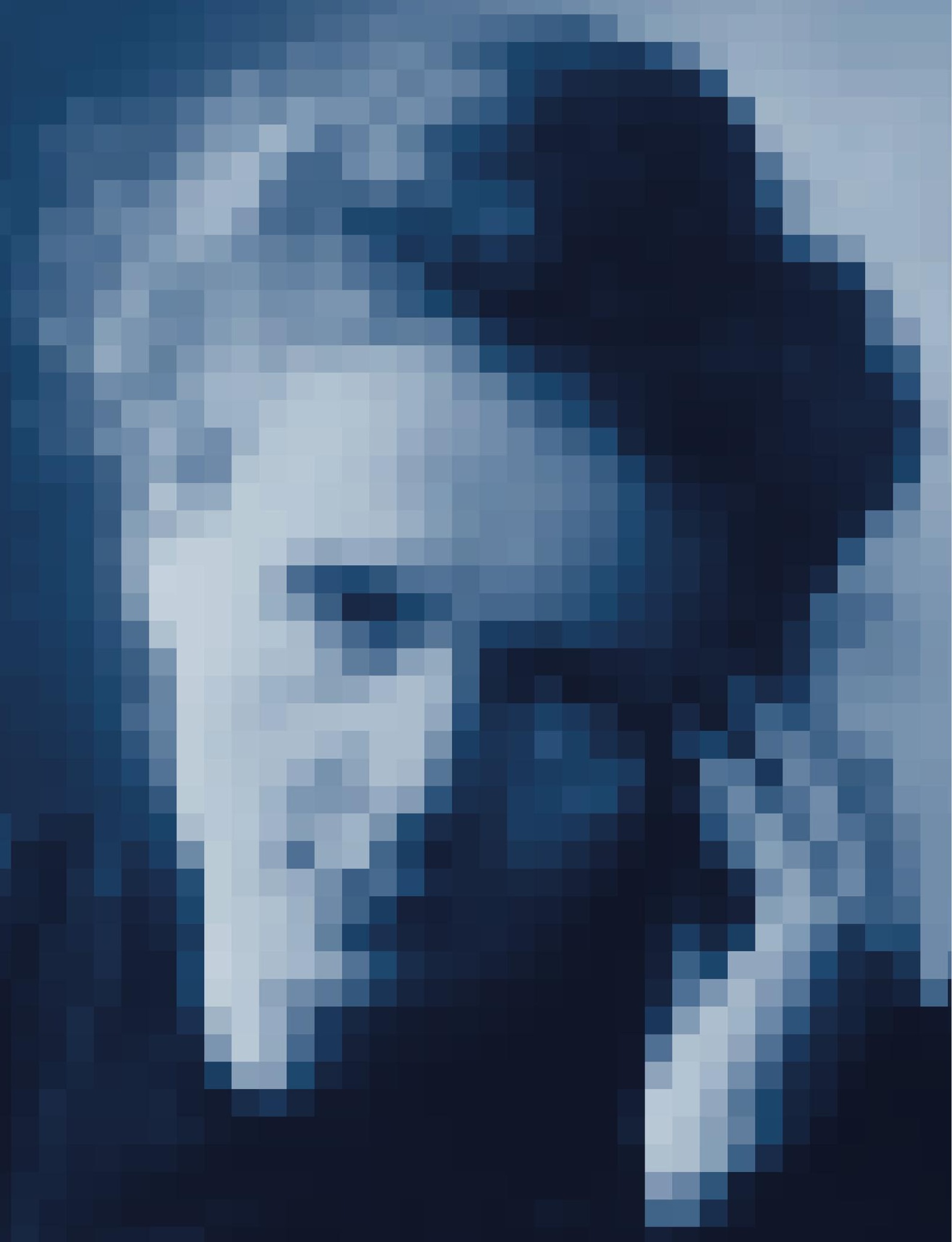




\section{CHAPTER 9}

\section{SUMMARY}

SAMENVATTING 


\section{Summary}

The studies described in this thesis directly or indirectly focused on the applicability of Cerenkov luminescence imaging (CLI) using gallium-68 prostate specific membrane antigen ( $\left.{ }^{68} \mathrm{Ga}-\mathrm{PSMA}-11\right)$ as a tool for margin assessment during prostate cancer ( $\mathrm{PC}$ ) surgery.

Part I of this thesis is focussed on the requirements for accurate intraoperative margin assessment in PCa surgery. To become familiar with the different players in the field, we have performed a systematic review on intraoperative technologies which perform margin assessment in PCa surgery in Chapter 2. In the last decade, five methods were mentioned, all based on optical imaging with different principles to distinguish tumour from benign tissue. The common disadvantages of these technologies were the long acquisition time and impediment of the surgical conditions (i.e., use of diathermy, presence of blood), thus making it difficult to be applied as a fast intraoperative assessment tool. Nevertheless, most technologies had a good sensitivity to detect the positive surgical margins (PSMs) as compared to the gold standard, histopathology. Still, it remained difficult for majority of these 174 technologies to be implemented in clinical practice, allowing room for improvement or development of alternative strategies.

Chapter 3 demonstrated the technical performance of CLI using ${ }^{68} \mathrm{Ga}$ in comparison to fluorine-18 $\left({ }^{18} \mathrm{~F}\right)$. From this in vitro study we concluded that the light yield is linear to the activity concentration, in which ${ }^{68} \mathrm{G}$ a showed a roughly 22 times higher light yield when compared to ${ }^{18} \mathrm{~F}$, while using the same activity level. Subsequently, the minimal activity concentration of ${ }^{68} \mathrm{Ga}$ which can be detected was lower, meaning that one can either image faster or inject a lower dosage of ${ }^{68} \mathrm{Ga}$. The improved light yield of ${ }^{68} \mathrm{Ga}$, caused by the higher positron energy, was at the expense of the spatial resolution of the $\mathrm{CLI}$, though still acceptable. Based on the aforementioned results an ex vivo clinical study using ${ }^{68} \mathrm{Ga}$-PSMA-11 for intraoperative margin assessment was deemed feasible.

Part II of this thesis explored whether ${ }^{68} \mathrm{Ga}-\mathrm{PSMA}-11 \mathrm{PET} / \mathrm{CT}$ in patients with primary PCa can be used to obtain additional knowledge for intraoperative CLI application, with regard to the tumour location, uptake intensity and kinetics. In Chapter 4 we described a prospective study on the repeatability of ${ }^{68} \mathrm{Ga}-\mathrm{PSMA}-11 \mathrm{PET} / \mathrm{CT}$ in patients with primary prostate cancer with a four-week interval between two scans. 
The repeatability coefficient of the primary prostate lesion was $18 \%$ for $S U L_{\text {peak }}$ and $22 \%$ for SUL $L_{\text {max }}$ (standardized uptake value corrected for lean body mass (SUL)). This study showed that in some patients there were slight visual differences in lesion uptake on PET, none of them being clinically relevant. We concluded from this study that ${ }^{68} \mathrm{Ga}$-PSMA-11 uptake on PET/CT in primary prostate cancer is repeatable. Consequently, ${ }^{68} \mathrm{Ga}-\mathrm{PSMA}-11 \mathrm{PET} / \mathrm{CT}$ can be used preoperative to select patients for the main CLI study, as preoperative uptake was considered a fair indicator for intraoperative uptake. Additionally, this implied that PET/CT with ${ }^{68} \mathrm{Ga}-\mathrm{PSMA}-11$ might potentially be used for response monitoring in the primary setting. However, the response criteria developed for ${ }^{18} \mathrm{~F}-\mathrm{FDG}$ might not hold for ${ }^{68} \mathrm{Ga}-\mathrm{PSMA}-11$.

In Chapter 5 dynamic PET/CT scans were performed using ${ }^{68} \mathrm{Ga}$-PSMA-11 to investigate the timing for $\mathrm{CLI}$ imaging. The early dynamics proved highly repeatable, given the small within subject coefficient of variation. An uptake plateau was reached for the iliac artery and gluteal muscle 5 minutes post-injection (p.i.). In some patients tumour uptake reached a plateau at 5 minutes p.i., whereas in others the uptake kept increasing over time and did not reach a plateau up to 60 minutes p.i. The hypothesis was that this difference between the uptake patterns is caused by tumour volume, in which in large tumours the uptake did not reach a plateau over time. Though not fully understood, the information might be beneficial for personalized dosing and radionuclide therapies in patient with low-volume tumour load. For the CLI study, we gained knowledge that at 5 minutes p.i. CLI imaging of the tumour is theoretically possible.

Part III of this thesis described the actual intraoperative use of ${ }^{68} \mathrm{Ga}-\mathrm{PSMA}-11 \mathrm{CLI}$, as margin assessment technology in a prospective clinical study. The initial feasibility of this technique was assessed in Chapter 6. The acquisition settings of the technique were optimized for the intraoperative ex vivo setting. CLI was able to accurately detect tumour in cleaved prostate specimens. In addition, the PSMs on the prostate surface were correctly identified on CLI. However, CLI detected false positives in two patients as well, however after histopathological assessment it appeared that the tumour to the inked margin distance was only $0.1 \mathrm{~mm}$. With respect to the safety aspects of $\mathrm{CLI}$, the radiation exposure to staff was within acceptable limits. The scrub nurse next to the patient was exposed to the highest dose $(0.016$ mSv per procedure). The exposure to other staff was at least 3 times lower. In conclusion, the study showed that intraoperative $\mathrm{CLI}$ is a feasible technique. After this interim analysis, the main study was reported in Chapter 7. This chapter elab- 
orated on the accuracy of CLI compared to histopathology and characterizes the novel identified bioluminescence signal. Solely looking at the presence of a hotspot on CLI (yes/no), the agreement between CLI and histopathology was only $31 \%$. Disagreement occurred profoundly at the base. When applying visual image interpretation, by given hotspots a Likert score, the agreement improved to $59 \%$. When close margins on histopathology (tumour $\leq 1 \mathrm{~mm}$ from the inked surface) were also included as positive, the agreement was $82 \%$. The remaining mismatch between $\mathrm{CLI}$ and histopathology was likely caused by the use of diathermy during surgery. Diathermy induced a bioluminescence that hampered the visualization of the PSMs. Though, there was no definite solution found yet to overcome this problem, there are still various clinically relevant applications of CLI to explore. 


\section{Samenvatting}

De studies beschreven in dit proefschrift zijn direct of indirect verbonden aan de toepassing van ${ }^{68} \mathrm{Ga}-P S M A-11$ met 'Cerenkov luminescence imaging' (CLI), als methode om de resectieranden te beoordelen tijdens prostaatkankerchirurgie.

Deel I van dit proefschrift focust op de eisen die gelden voor een accurate intraoperatieve beoordeling van de snijranden tijdens prostaatkankerchirurgie. In Hoofdstuk $\mathbf{2}$ is een systematische literatuurreview uitgevoerd, waarbij verschillende intraoperatieve technieken zijn vergeleken die de snijranden bij prostaatkanker beoordelen. In het afgelopen decennium is er gebruik gemaakt van vijf technieken, allemaal gebaseerd op optische beeldvorming, maar op basis van verschillende natuurkundige principes om de tumor van benigne weefsel te onderscheiden. De onderzochte technieken hebben allen een goede sensitiviteit om positieve snijranden te detecteren, waarbij er vergeleken werd met de gouden standaard, histopathologie. De gezamenlijke nadelen van deze technieken waren de relatief langere acquisitietijden en de beperking door de omstandigheden tijdens de operatie (o.a. gebruik diathermie, aanwezigheid bloed). Er is dus nog ruimte is voor verbetering, evenals de mogelijkheid tot ontwikkeling van alternatieve strategieën voor het intraoperatief beoordelen van de snijranden bij prostaatkanker.

Hoofdstuk 3 beschrijft de technische evaluatie van CLI, waarbij het gebruik van gallium-68 $\left({ }^{68} \mathrm{Ga}\right)$ werd vergeleken met fluor-18 $\left({ }^{18} \mathrm{~F}\right)$. Uit deze in vitro studie konden we concluderen dat de signaalopbrengst van de CLI lineair is met de activiteitconcentratie, waarbij ${ }^{68} \mathrm{Ga}$ ongeveer 22 keer meer lichtopbrengst heeft dan ${ }^{18} \mathrm{~F}$ bij dezelfde activiteitconcentratie. Ook de minimale activiteit concentratie van ${ }^{68} \mathrm{Ga}$ dat nog gedetecteerd kan worden was lager, resulterend in de voordelen van een lagere te gebruiken hoeveelheid activiteit of het inkorten van de acquisitietijd. De hogere lichtopbrengst ging ten koste van de spatiële resolutie, dit als gevolg van de grotere positronendracht van ${ }^{68} \mathrm{Ga}$. Gebaseerd op de genoemde resultaten, leek een klinische studie naar het ex vivo gebruik van CLI met ${ }^{68} \mathrm{Ga}-\mathrm{PSMA}-11$ tijdens prostaatkankerchirurgie haalbaar.

Deel II van dit proefschrift onderzocht of ${ }^{68} \mathrm{Ga}$-PSMA-11 PET/CT bij patiënten met primair prostaatcarcinoom kan worden ingezet om informatie te vergaren vóór de intraoperatieve toepassing van CLI. In Hoofdstuk $\mathbf{4}$ hebben we, door middel van een prospectieve studie, gekeken naar de reproduceerbaarheid van opname patro- 
nen op twee opeenvolgende ${ }^{68} \mathrm{Ga}$-PSMA-11 PET/CT-scans bij patiënten met primair prostaatcarcinoom binnen een interval van 4 weken. De reproduceerbaarheid coefficiënt (RC) van de primaire laesies was $18 \%$ voor de $S U L_{\text {peak }}$ en $22 \%$ voor $S U L_{\text {max }}$ ('standardized uptake value' gecorrigeerd voor de vetvrije massa (SUL)). Deze studie liet zien dat bij sommige patiënten er visuele verschillen waren aangaande de laesie-opname op de PET/CT, echter was dit in alle gevallen niet klinisch relevant. We concludeerden uit deze studie dat de opname op de ${ }^{68} \mathrm{Ga}-\mathrm{PSMA}-11$ PET/CT bij patiënten met primair prostaatcarcinoom reproduceerbaar is. ${ }^{68} \mathrm{Ga}-\mathrm{PSMA}-11 \mathrm{PET} / \mathrm{CT}$ kon dus ingezet worden om patiënten te selecteren voor de CLI-studie, waarbij de preoperatieve opname een goede indicatie was voor de intraoperatieve opname. Verder zou ${ }^{68} \mathrm{Ga}$-PSMA-11 PET/CT gebruikt kunnen worden voor respons monitoring in de primaire setting. Echter te verwachten is dat de respons criteria, die ontwikkeld zijn voor ${ }^{18} \mathrm{~F}-\mathrm{FDG}$, niet opgaan voor ${ }^{68} \mathrm{Ga}-\mathrm{PSMA}-11$.

In Hoofdstuk 5 waren er dynamische ${ }^{68} \mathrm{Ga}$-PSMA-11 PET/CT-scans geanalyseerd om de timing voor CLI-beeldvorming vast te stellen. De vroege dynamiek van het radiofarmacon bleek reproduceerbaar, aangezien er maar een kleine variatie was tussen opname patronen op de scans. De opname in de arterie iliaca vertoonde een 178 plateau 5 minuten na injectie, evenals de musculus gluteus. Bij sommige patiënten liet de tumoropname na 5 minuten ook een plateau zien, in tegenstelling tot enkele patiënten waarbij de tumoropname bleef stijgen over een periode van 60 minuten na injectie. Het lijkt aannemelijk dat deze verschillen in opnamepatronen worden veroorzaakt door het tumorvolume, waarbij in grotere tumoren de PSMA-opname blijft stijgen. Hoewel het principe hierachter nog niet volledig duidelijk is, kan deze informatie waardevol zijn voor het personaliseren van doseringen bij radionuclide therapie in prostaatkanker. Met het oog op de klinische CLI-studie zijn we erachter gekomen dat er 5 minuten na injectie voldoende opname in de tumor was waardoor afbeelden met CLI theoretisch mogelijk is.

In deel III van dit proefschrift werd het intraoperatieve gebruik van ${ }^{68} \mathrm{Ga}-\mathrm{PSMA}-11$ $\mathrm{CLI}$ onderzocht in een prospectieve klinische studie als modaliteit om resectieranden te beoordelen. De initiële haalbaarheid van deze technologie in de klinische praktijk werd besproken in Hoofdstuk 6. Het acquisitieprotocol werd geoptimaliseerd voor de ex vivo situatie tijdens chirurgie. Daarnaast was het mogelijk om met CLI tumorweefsel te detecteren in de gekliefde prostaat. Daarbij werden ook positieve snijranden (tumor in de geïnte snijrand) correct geïdentificeerd. Echter werden er in 2 patiënten ook foutpositieve bevindingen gedaan, waar bij nader 
onderzoek bleek dat de afstand van de tumor tot de geïnte rand maar $0.1 \mathrm{~mm}$ bedroeg. Niet onbelangrijk: de stralingsdosis van het operatiepersoneel was acceptabel voor de huidige toepassing De assistent die steriel stond (dichtbij de patiënt) werd blootgesteld aan de hoogste dosis, 0.016 mSv per procedure. Overig aanwezig personeel werd blootgesteld aan minder dan 1/3 van deze stralingsdosis. Op basis van de initiële analyses concludeerden we dat CLI een haalbare techniek is die verder gevalideerd werd.

In Hoofdstuk 7 werd de accuratesse van de CLI belicht ten opzichte van histopathologie, en daarnaast werd de eerder geobserveerde bioluminescentie gekarakteriseerd. Wanneer men kijkt naar het al dan niet aanwezig zijn van een signaal op CLI, was de overeenkomst tussen CLI en histopathologie maar 31\%. Het verschil was het grootste aan de basale zijde van de prostaat. Wanneer visuele interpretatie van de CLI afbeeldingen werd toegepast door middel van het toekennen van een Likert score, steeg de overeenkomst naar 59\%. Zodra er marges binnen $1 \mathrm{~mm}$ van de geïnte rand werden gedefinieerd als positief, werd de overeenkomst $82 \%$. Verschil tussen CLI en histopathologie wordt hoogstwaarschijnlijk veroorzaakt door het gebruik van diathermie tijdens de operatie. Diathermie induceerde bioluminescentie die de detectie-accuratesse van positieve snijranden beperkte. Ondanks dat er is nog geen definitieve oplossing is gevonden voor dit probleem, zijn er klinisch relevante toepassingsmogelijkheden van CLI die onderzocht kunnen worden. 


\section{APPENDICES}

LIST OF PUBLICATIONS

LIST OF AFFILIATIONS

DANKWOORD

ABOUT THEAUTHOR 


\section{List of Publications}

\section{PEeR REVIEWED PUBLICATIONS IN THIS THESIS}

1. olde Heuvel J, de Wit-van der Veen BJ, Vyas KN, Tuch DS, Grootendorst MR, Stokkel MPM, Slump CH. Performance evaluation of Cerenkov luminescence imaging: a comparison of ${ }^{68} \mathrm{Ga}$ with ${ }^{18} \mathrm{~F}$. EJNMMI Phys. 2019;6:17.

2. olde Heuvel J, de Wit-van der Veen BJ, Huizing DM, van der Poel HG, van Leeuwen PJ, Bhairosing PA, Stokkel MPM, Slump CH. State-of-the-art Intraoperative Imaging Technologies for Prostate Margin Assessment: A Systematic Review. Eur. Urol. Focus. 2020; Epub ahead of print

3. olde Heuvel J, de Wit-van der Veen BJ, van der Poel HG, Bekers EM, Grootendorst MR, Vyas KN, Slump CH, Stokkel MPM, ${ }^{68} \mathrm{Ga}-\mathrm{PSMA}$ Cerenkov luminescence imaging in primary prostate cancer: first-in-man series. Eur. J. Nucl. Med. Mol. Imaging. 2020;47:2624-2632

4. olde Heuvel J, de Wit-van der Veen BJ, Donswijk ML, Slump CH, Stokkel MPM. Dayto-day variability of $\left[{ }^{68} \mathrm{Ga}\right] \mathrm{Ga}-\mathrm{PSMA}-11$ accumulation in primary prostate cancer: effects on tracer uptake and visual interpretation. EJNMMI Res. 2020;10:132

5. olde Heuvel J, de Wit-van der Veen BJ, Sinaasappel M, Slump CH, Stokkel MPM (2021) Early differences in dynamic uptake of 68Ga-PSMA-11 in primary prostate cancer: A test-retest study. PLoS ONE 16(2): e0246394.

\section{OTHER PEER-REVIEWED PUBLICATIONS}

1. Grob ATM, olde Heuvel J, Fütterer JJ, Massop D, van Nieuwenhoven AL, Simonis FFJ, van der Vaart CH. Underestimation of pelvic organ prolapse in the supine straining position, based on magnetic resonance imaging findings. Int. Urogynecol. J. 2019;30:1939-1944.

2. Collamati F, van Oosterom MN, De Simoni M, Faccini R, Fischetti M, Mancini Terracciano C, Mirabelli R, Moretti R, olde Heuvel J, Solfaroli Camillocci S, van Beurden $F$, van der Poel HG, Valdes Olmos RA, van Leeuwen PJ, van Leeuwen FWB, Morganti S. A DROP-IN beta probe for robot-assisted ${ }^{68} \mathrm{Ga}-\mathrm{PSMA}$ radioguided surgery: first ex vivo technology evaluation using prostate cancer specimens. EJNMMI Res.2020;10:92

\section{NON-PEER REVIEWED PUBLICATIONS}

1. olde Heuvel J, de Wit-van der Veen B, van der Poel HG, Stokkel MPM, Slump $\mathrm{CH}$. Intraoperative specimen assessment in prostate cancer surgery using $\mathrm{Ce}$ renkov luminescence imaging. 2020, Proc. of SPIE Vol. 11224, 1122407-1. 


\section{List of Affiliations}

Judith olde Heuvel

Technical Medical Centre, University of Twente

Department of Nuclear Medicine, Netherlands Cancer Institute

Berlinda J. de Wit-van der Veen

Department of Nuclear Medicine, Netherlands Cancer Institute

Marcel P.M. Stokkel

Department of Nuclear Medicine, Netherlands Cancer Institute

Daphne M.V. Huizing

Department of Nuclear Medicine, Netherlands Cancer Institute

Maarten L. Donswijk

Department of Nuclear Medicine, Netherlands Cancer Institute

Michiel Sinaasappel

Department of Physics, Netherlands Cancer Institute

Pim J. van Leeuwen

Department of Urology, Netherlands Cancer Institute

Henk G. van der Poel

Department of Urology, Netherlands Cancer Institute

Elise M. Bekers

Department of Pathology, Netherlands Cancer Institute

Patrick A. Bhairosing

Scientific Information Service, Netherlands Cancer Institute

Cornelis H. Slump

Technical Medical Centre, University of Twente

Maarten R. Grootendorst

Lightpoint Medical Ltd.

David S. Tuch

Lightpoint Medical Ltd.

Kunal N. Vyas

Lightpoint Medical Ltd. 


\section{DANKWOORD}

Het zit erop! Klaar! Hoewel ik heb genoten van het onderzoek doen, de collega's, alle ontwikkelingen die ik heb mogen meemaken en kansen die ik heb gekregen, voelde het soms alsof ik alleen op een eilandje zat. Gelukkig waren er vaak bootjes die kwamen aanmeren voor inhoudelijke en mentale support. Die mensen zou ik graag willen bedanken.

Beginnend bij de (co)-promotoren, de KLM (Kees, Linda, Marcel). Die vanaf verschillende hoogtes mijn vorderingen bijhielden, bijstuurden en altijd bevlogen enthousiast waren. Beste Kees, ik ben ontzettend blij dat jij mijn promotor bent geweest. Je nuchtere blik, je grapjes en interesse in alles wat ik deed hebben mij erg geholpen in het PhD-proces. Daarnaast hebben we onze grote vriend Hora Finita overwonnen en ben ik blij dat ik van jou de mogelijkheid heb gekregen om wat colleges te geven. Beste Marcel, bedankt voor de mogelijkheid om op de nucleaire geneeskunde mijn PhD te doen en alle kansen die je mij daar hebt gegeven. Jij was positief, als ik het zelf even niet meer wist. Ik vond het heel fijn dat jouw deur altijd open stond voor een korte vraag of natuurlijk de befaamde handtekening.

184 Best Linda, ik heb enorm veel bewondering voor al je kennis en kunde. Je wist me door onze wekelijkse meetings vaak weer nieuwe energie en motivatie te geven voor de projecten. Gek genoeg was ik altijd blij als mijn artikelen rood terugkwamen, omdat ik wist dat het stuk hierdoor nog beter werd en dat me weer kon bijleren in het wetenschappelijk schrijven. Maar bovenal was samenwerken met jou erg gezellig en heb ik genoten van de gezamenlijke koffiemomentjes, lunches en congressen.

Ik wil de leden van de beoordelingscommissie prof. dr. I.A.M.J. Broeders, prof. dr. J.J. Fütterer, prof. dr. L.F. de Geus-Oei, prof. dr. R. de Bree en dr. D.E. Oprea-Lager bedanken voor het lezen en beoordelen van dit proefschrift.

Beste Baris Karakullukcu en Uulke van der Heide, bedankt dat jullie mijn onafhankelijke ○○A-begeleiders wilden zijn. En dat jullie naast het bewaken dan de voortgang van het project, ook graag mee dachten over de inhoud.

Dit hele proefschrift wat niet van de grond gekomen zonder de hulp van de patiënten, die bereid waren om mee te doen aan mijn onderzoek. Heel erg veel dank voor jullie vertrouwen in de wetenschap en de interesse in wat er in de wetenschap allemaal gebeurd. 
Daarnaast de verpleegkundig specialisten van de urologie, die mee hebben geholpen met de inclusie van de patiënten. Beste Corinne, Erik, Jeroen, Jolien en Saskia dank voor al jullie hulp, het aannemen van informed consents en de uitleg van de studies. Beste Esther, Henk en Pim, dank voor jullie flexibiliteit tijdens de RALP'en, het meedenken en schrijven aan artikelen. En natuurlijk het 'over het beeldscherm heen kijken tijdens het MDO'-moment om te vragen of dit een geschikte patiënt zou zijn voor de CLIPPS-studie.

Alle OK-assistenten en anesthesiemedewerkers van team robot, in het bijzonder Janneke Prins, heel erg dank voor jullie hulp tijdens de CLIPPS-studie. Ik waardeerde het heel erg dat jullie meedachten over de studie en hoe de logistiek goed opgezet kon worden. Daarnaast waren jullie altijd bereid om mee te werken en was het natuurlijk gezellig om aanwezig te zijn op OK.

De afdeling pathologie, in het bijzonder Maarten en Kelly. Bedankt voor de training om te leren hoe je een prostaat inkt en klieft en voor de hulp bij de studieopzet. Daarnaast natuurlijk Elise, voor het beoordelen van alle prostaatslides, het meten van de afstanden en het meeschrijven aan de artikelen.

Tonnie en Bregje van bureau opname, voor het inplannen/verzetten/vervroegen/ verlaten van alle patiënten. Dank voor jullie flexibiliteit.

This thesis is improved by the collaboration with Lightpoint Medical. I've learned a lot from all of you regarding image analysis, CLI in general and the critical review of my papers. Next, I really enjoyed the cups of coffee during the conferences and our annual meetings. Thank you very much Maarten, Kunal, David and Claire.

Jolanda, bedankt voor het maken en regelen van vele dingen op de UT, als externe PhD kon ik altijd rekenen op jou. Dank daarvoor. Beste Nicole, allereerst heb ik altijd genoten van onze gesprekken op de tuinhuisgang en bij het koffiezetapparaat. Daarnaast was ik blij dat jij kon helpen met gaatjes vinden in drukke agenda's en dat jij dacht aan het verzorgen van de koffie tijdens meetings als ik dat vergeten was.

Prostate-pizza-people, thanks a lot for all our Wednesday afternoon meetings. I've learned that there is a whole different field out there which investigates prostate cancer, and I admire the hard work you do. Next, I learned a new vocabulary with fancy words, like ChIP-seq, CRISPR Cas and fluorescence-activated cell sorting. 
Dan natuurlijk de afdeling, ik had me geen leukere afdeling kunnen bedenken voor tijdens mijn PhD. Jullie zijn een superleuk team, ik heb me altijd heel welkom gevoeld. Jullie waren altijd bereid om voor mij spuiten op te trekken, wel of geen Lasix toe te dienen en het meedenken over de scanprotocollen van de PRET-studie. In het bijzonder Lyandra, die zowel voor de PRET als de CLIPPS-studie altijd haar best heeft gedaan voor het zoeken naar plekjes op de Gallium planning. Daarnaast heb ik me altijd erg vermaakt tijdens pauzes, vrijdagmiddagborrels en afdelingsuitjes. Natuurlijk ook bedankt voor jullie magen, die altijd bereid waren om mijn baksels op te eten. Aafke, Arida, Armina, Cas, Chantal, Chelvi, Cheryl, Christel F, Colinda, Daniëlle, Desiree, Emilie, Esther, Gabi, Hajar, Ilse, Ingrid, Jelmer, Jeroen E, Jonne, Josette, Kirsten, Lyandra, Maaike, Marieke, Marien, Mariëtte, Marintha, Marle, Miena, Myriam, Nancy, Natascha, Saskia, Sharon, Simone, Sophie, Ted. Toch nog in het bijzonder de borrelclub (jullie weten wel wie jullie zijn), bedankt voor de prachtige (en soms vage) herinneringen aan de rollende keukens, het feest van Joop, TonTon club, de Biertuin, Radion en al die andere momenten. Ik hoop dat we dit ook na mijn PhD dit kunnen blijven doen.

Mijn dank gaat ook uit naar de NG-artsen, Bernies, Christel, Erik, Emilia, Karen, Marcel, Maarten, Wouter en Zing voor het aanvragen van de scans, al jullie kennis en de praatjes in de gang. Maarten, voor jou nog speciale dank voor het beoordelen van de studie scans samen met Marcel, en het meeschrijven aan de test-retest paper. Martine en Jeroen, bedankt voor het beantwoorden van mijn apotheek-vragen.

Dear Tuinhuis people, I really liked being part of the radiology gang. I've learned a lot about non-work related stuff from you during our lunchbreaks, from bitcoins, to food, to newspapers and family-in-laws. Especially enjoyed our OOA-retreat in Renesse, secret Sinterklaas and the NKI summer parties. Nog een woordje extra voor Paula, Femke en Judith bedankt voor de vele persoonlijke gesprekken over Slack/in deuropeningen/bij de koffieautomaat.

De mede-eilandbezitters, lieve (ex-)PhD'ers, Daan, Daphne, Else, Hanna, Hinke en Jeske. Zonder jullie steun was dit boekje er niet gekomen. Daarnaast was het altijd erg gezellig en hebben we mooie momenten beleefd op congressen en borrels. Daan, jij was vaak de rust en de realist in de kamer, ik heb veel bewondering voor je creativiteit en intelligentie. Als ik iets vroeg aan jou, werd er naast een antwoord op mijn vraag vaak ook nog een nieuw onderzoek idee geopperd. Lieve

\section{Dankwoord}


Daphne, ik heb echt heel veel geluk gehad dat jij mijn roomie was voor bijna 3 jaar. Je had altijd een luisterend oor, een oplossing voor PhD problemen en zorgde ervoor dat er genoeg cappuccino momentjes waren. Sorry voor alle momenten dat ik weer eens niet te genieten was, en dankjewel dat jij altijd lief en geduldig bleef. Lieve Else, dank voor al je enthousiasme en het feit dat je overal over wilde meedenken. En de gezelligheid als theeleut tussen de koffiedrinkers. Lieve Hanna, als arts tussen alle technische geneeskundigen was ik altijd blij met jouw dokter blik. Daarnaast was ik altijd blij als jij me weer meesleurde naar het heilig half uur, want als ik er was vond ik het altijd super interessant. Lieve Hinke en Jeske, wat ben ik blij dat jullie mijn mede kamergenoten waren het laatste jaar. Ik heb erg met jullie gelachen en geklaagd. En vond het leuk om uit te leggen over de 'plaatjes', zoals apothekers scans blijkbaar noemen.

Gelukkig was er nog leven naast een PhD en mijn lieve vrienden hielpen me daar graag aan herinneren. Jultiem zorgde voor gezelligheid, gekke knutselprojecten en Pinksterweekendjes. De achtste hemel, door jullie ben ik altijd up-to-date met de nieuwste spellen en escape rooms. Ik ben elke keer weer blij dat ik bij jullie in huis heb gewoond en we nog steeds contact hebben. High Thai in my pocket/youtit and the sparkly sparks, thanks you oh so normal grandma's on the dancefloor. Dispuut bRAXIS, jullie zorgen ervoor dat ik als NuKuBu me soms weer even student voel, al is het een oude student. Lieve Anouk, Moniek en Niki, fijn dat jullie nog steeds mijn vriendinnen willen zijn, ook al praatte ik eerst over baarmoederverzakkingen en nu over prostaten. Gelukkig kan ik alle andere onderwerpen ook met jullie bespreken, onder het genot van 43 en baksels. Moniek, bedankt voor je vertrouwen in mij dat ik de prostaat niet zou laten vallen op OK, mede daardoor is het ook nooit gebeurd. Lieve Niki, wat ben ik blij dat jij mijn paranimf wil zijn. Ik ben heel dankbaar voor onze vriendschap, het vertrouwen en natuurlijk je schaterlach en humor. Ten tweede, als lotgenoot omdat je weet wat ik doormaak.

Lieve Berber, dank dat jij dit prachtige proefschrift hebt willen vormgeven. Ik ben blij dat we dit samen hebben kunnen doen. Maar nog blijer ben ik dat we na al die jaren nog steeds bevriend zijn en dat jij mijn broodbakbuddy bent.

De Ardenianen, (Hanneke, Harm, Lara, Martijn, Mattiènne, Ruud, Stijn en Yvonne) wat ben ik blij dat 'functies van een veranderlijke' ons op wonderbaarlijke wijze allemaal heeft verbonden. Ik ben heel erg blij met onze vriendengroep en ons jaarlijkse Ardennen weekend. Hanneke voor je oprechte interesse en als spelletjes- 
maatje. Harm, voor het voeren van maatschappij/klimaat discussies met de groep. Martijn en Stijn, dank dat jullie me helpen herinneren dat een PhD ook maar werk is. Lara, voor het feit dat je sinds de basisschool al niet van mijn zijde wijkt. Claire en Mattiènne, jullie zijn mijn tweede thuis, altijd bereid om te zorgen dat ik even niet aan mijn PhD denk. Wacht maar tot ik jullie buurvrouw ben. Ruud, dank voor het meelezen, het langer maken van mijn zinnen en het structureren van mijn gedachtes als ik het zelf even niet meer wist. Naast de schrijfuurtjes ben ik ook heel blij met jou als mijn trouwe vriend. Yvonne, met jou is het leven toch een stuk gezelliger, ik ben blij dat je naar mijn gezeur wil luisteren en dat je altijd bereid bent om 'koffie' met mij te drinken.

Lieve (schoon)-familie, bedankt voor jullie betrokkenheid en gezelligheid als we samen zijn. Lieve opa en oma, ook al hebben jullie geen idee wat ik doe, jullie vinden het alsnog prachtig. Ik vind het heel lief dat jullie stukjes uit de krant knippen als er iets medisch technisch in staat wat ik misschien leuk vind. Als ik op bezoek ben is de lach altijd aanwezig en de van der Poel om de hoek. Ik ben blij dat ik deze dag met jullie mag meemaken en opa en oma olde Heuvel zijn er vast in gedachten bij.

Lieve Papa en Anja, bedankt voor jullie oprechte interesse in mijn onderzoek, ik vind het knap dat jullie vaak een poging waagden om de artikelen te 'lezen' die ik had geschreven. Natuurlijk ook dank voor het corrigeren van mijn Nederlandse samenvatting en jullie steun in alles wat ik doe.

Lieve Mama en Rob, bedankt voor het luisterende oor en alle adviezen. Ook al lijkt het soms dat ik er niks mee doe, het blijft toch ergens hangen in het koppie. Lieve Mama, bedankt voor al je vertrouwen in mij, je liet me altijd geloven in mijzelf en ik weet dat je achter me staat.

Lieve Kirsten, Kisik, er bestaat geen betere zus dan jij. Jij kent me beter dan wie dan ook en zorgt gevraagd of ongevraagd voor een spiegel. Ik ben heel blij dat jij mijn (para)nimf wil zijn, ook al zei je ja voordat je enig idee had waar je ja tegen zei.

Lieve Mariëtte, allerliefste Jetje, jij die alles van begin tot het einde hebt meegemaakt, van de worstelingen tot de hoogtepunten, van de tranen tot de publicatie-traktaties. Wat het ook was, je was er altijd voor mij, ook al was ik niet te genieten (sorry). Duizendmaal dank! Ook aan de harige viervoeters Lore \& Trix, die 
met hun pootjes op het toetsenbord verantwoordelijk zijn voor de spelfouten in dit proefschrift. Lieve Jetje, wat ben ik blij dat ik met jou de 'mystery of love' mag ontdekken. 


\section{About the author}

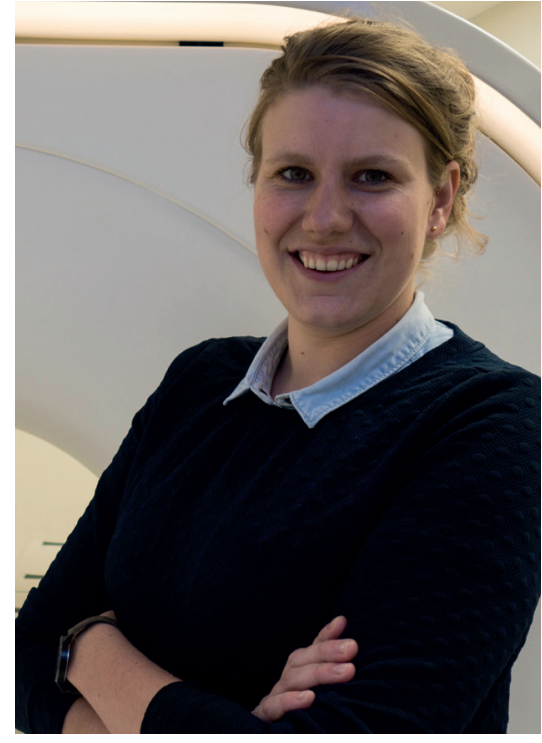

Judith olde Heuvel was born on the $4^{\text {th }}$ of January 1992 in Hoogeveen, The Netherlands. In 2010 she graduated from high school (Bataafse Kamp, Hengelo), after which she started studying Technical Medicine at the University of Twente in Enschede. She obtained her Bachelor degree in 2013, subsequently started the Master track of Technical Medicine - Medical Imaging and Interventions. After the first year of the master, she went on study tour to India to investigate medical tourism, for which several hospitals, medtech companies and universities were visited. Thereafter, she continued her studies and performed several research internships at different hospitals and departments throughout the Netherlands and the UK: Biomedical Engineering and Physics (Amsterdam UMC), rheumatology (UMC Utrecht), gynaecology (Radboud UMC) and surgical oncology (Guy's hospital, London).

She performed her graduation internship at the gynaecology department in the UMC Utrecht, where she evaluated pelvic organ prolapse using upright MRI. With this research she obtained her master degree and was awarded with the Prof. Vooijs award concerning the most relevant technical medicine master thesis of the year 2017.

Judith started her PhD-reserach at the nuclear medicine department in the Antoni van Leeuwenhoek in August 2017, which resulted in this thesis. During her PhD, she was an active member of the public relations committee of the Nederlandse Vereniging voor Technische Geneeskunde (NVVTG) and a volunteer at the 'spelletjesavond' for the elderly with dementia. In her spare time she likes to make bread and bake cakes for the nuclear medicine department.

At the moment of writing, Judith is looking for a job in which she can collaborate and educate professionals with different areas of expertise to improve medical technology for clinical care. 

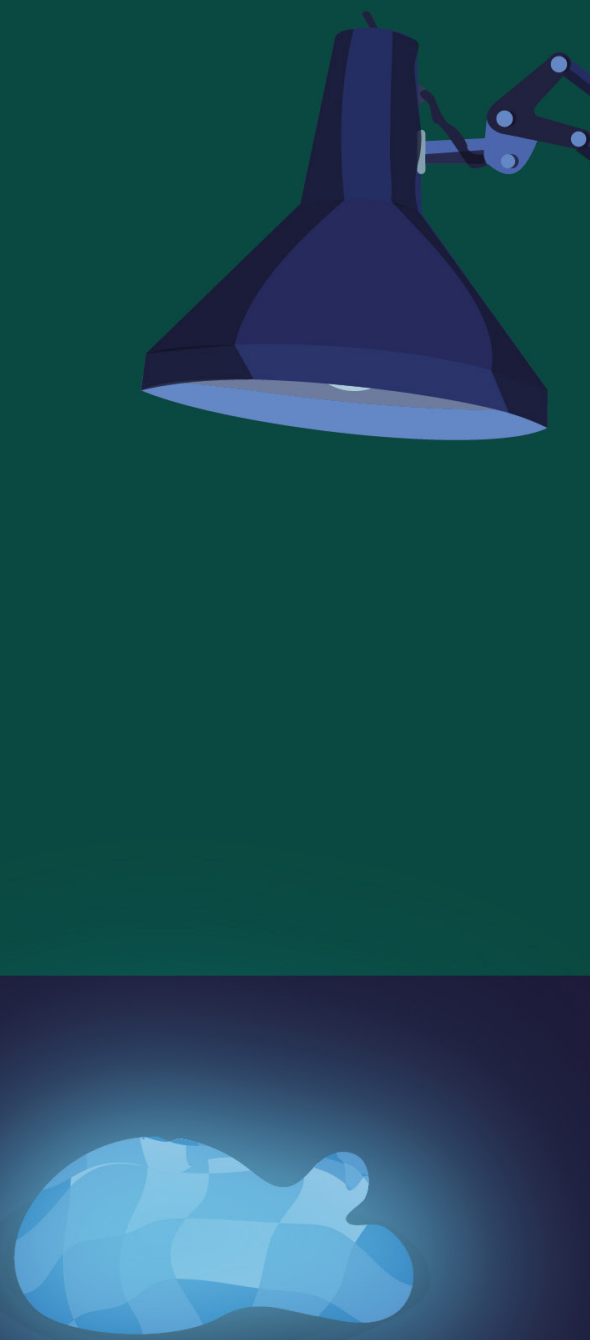